\title{
Flame aerosol synthesis of nanostructured materials and functional devices:
}

\section{Processing, modeling, and diagnostics}

\author{
Shuiqing Li ${ }^{1, *}$, Yihua Ren ${ }^{1}$, Pratim Biswas ${ }^{2}$, Stephen D. Tse ${ }^{3}$
}

\begin{abstract}
:
Manufacturing of nanostructured materials and functional devices offers many exciting opportunities for scientists and engineers to contribute substantially in renewable energy utilization, environmental compliance, and product development. In the past two decades, gas-phase flame synthesis has not only proved to be one of the most scalable and economical technologies for producing well-controlled nanostructured materials, including single metal-oxide, mixed-oxide nanocomposite, and carbon nanostructures, but also has been recognized as a new low-cost fabrication method of nano-devices. In this paper, we focus our review mainly on the recent trends in specific applications of flame aerosol synthesis in the last decade, e.g., usage of a substrate in stagnation geometry with controlled particle temperature-time history, application of external fields to control particle characteristics, development of advanced spray technique for doping synthesis of nanocomposites of multicomponent metal oxides or carbon-metal oxides, and fabrication of nanomaterial-based functional devices. For the possibility to improve the design and operation of flame aerosol reactors, in situ optical diagnostics for either gas phase or particle phase in flame field, along with multi-scale modeling and simulation employing gas-phase chemistry, population balance method, molecular dynamics, and nanoscale particle dynamics are summarized.
\end{abstract}

Key words: flame synthesis, functional materials, nano-devices, nanocomposite, population balance, optical diagnostics, molecular dynamics 


\section{Introduction}

Nanotechnology is expected to have major impacts in medicine, catalysis, electronics, and materials science. The basic science and engineering of nanoparticles (and nanomaterials increasingly in general) play a central role in many engineering fields, including energy (e.g. nanoenergetic or nanocatalytic materials, fossil fuel combustion, fly-ash formation), environment (e.g. air pollution, climate change), and biotechnology (e.g. medical diagnostics, drug delivery). A topic of significant interest in the nanomaterial field is synthesis of nanoparticles with specific properties. Extensive literature exists on synthesis methods to produce nanoparticles with desired coatings; mechanical, chemical, electric, and magnetic properties; hollow and solid cores; and various crystalline structures [1]-[5]. A second area of great interest deals with the aerosol formation of nanoparticles into larger assemblages or functional devices, as well as ways to control the self-assembly processes themselves [5]-[9].

Currently, many commodity nanoparticle products are made by flame aerosol processes, including carbon black (Cabot, Columbia, Degussa-Hüls), fumed silica (Cabot, Degussa-Hüls), pigmentary titania (DuPont, Ishihara, Millenium, Kerr-McGee, Cristal), and optical fibers (Corning, Heraeus, Lucent, Sumitomo), with production of millions of tons valued at over $\$ 15$ billion/year [5]. Compared to wet chemistry approaches (e.g., sol-gel), aerosol technology has proven advantageous for manufacture of commercial quantities of nanoparticles due to its high-throughput production, fast processing time, facilitating process design (manufacturing and collecting), and apparent simplicity (continuous one-step process) [5],[9]-[11]. Moreover, nanoparticles with complex compositions, such as most mixed oxide ceramics, can be manufactured more cheaply and with less environmental harm by aerosol technology 
than by wet-chemistry [9][12]. Though the practice of flame aerosol technology dates back to prehistoric times, as used for paintings on cave walls and in Chinese ink artwork, its R\&D importance to create new materials for catalysis, composites, and other applications have been recognized only in the last thirty years by visionaries such as Dr. Ulrich in 1971 [13][14], Dr. Formenti et al. in 1972 [15], and others [1].

\subsection{Current Status of Flame Aerosol Synthesis}

Flame aerosol synthesis, also termed as flame aerosol technology, can be categorized in two general arrangements depending on the precursor feeding condition, i.e., vapor-fed aerosol flame synthesis (VAFS), and liquid-fed aerosol flame synthesis (LAFS) (by using a sprayer or an atomizer). Nevertheless, it is classified as gas-gas combustion synthesis, since the nanoparticles form directly from the gas phase through the self-sustaining reaction of gaseous species [16]. In this paper, we intensively

focus on the flame aerosol synthesis of nanostructured metal oxides and their multicomponent composites, as well as various cabon nanostructures and carbon-metal oxide composites. The flame synthesis of nanostructed carbon materials involving heterogeneous growth on substrates, recently reviewed in [17], are not included here. In addition, the well-known self-propagating, high-temperature synthesis (SHS) process for materials synthesis, including gas-phase-facilitated solid-solid synthesis and gas-condensed-phase combustion synthesis, do not fit well within this classification and lie outside the scope of this review.

As shown in Fig. 1, the vapor-fed aerosol flame synthesis (VAFS) method (where combustion, decomposition, or hydrolysis of volatile precursors take place in supported hydrocarbon, hydrogen, or halide flames) is usually recognized as one of the classic gas-to-particle conversion processes. 
Particles form via the mechanism of homogenous (or spontaneously heterogeneous) nucleation of low-vapor-pressure species and then agglomerate/grow by the competing mechanism of collision-coalescence among the nascent nanoparticles [18]-[25]. The gas-to-particle conversion can be driven by two routes: (i) the chemical reaction of gaseous precursors (chemical route), and (ii) the physical cooling of hot vapor (physical route). Understandably, the flame aerosol reactor belongs to the chemical route because the involving chemical reactions transform precursor vapors to product vapors (with a chemical time scale of $\tau_{c h e m}=1 / k_{p}$, where $k_{p}$ is the coefficient constant of precursor reaction rate). The flame aerosol reactor shares similar routes with plasma, laser, and electrically-heated wall reactors, but do not require an additional source of energy for precursor conversion, presenting a rather low capital investment with proven scalability [1][24]. The key advantages of vapor-fed flame synthesis are the manufacturing of products with high purity, well-controlled crystallinity, ultrafine diameter, and narrow size distribution. Upon reaction, the product vapor becomes sufficiently supersaturated such that homogeneous nucleation occurs to form thermodynamically stable clusters, during which the low vapor pressures of these materials (e.g., $\mathrm{TiO}_{2}, \mathrm{Al}_{2} \mathrm{O}_{3}$ ) in the reaction zone present no energy barrier for the process. As shown in Fig. 1, once clusters are formed, they can grow by heterogeneous nucleation (also termed as surface growth due to the condensation of product vapor monomers) or by collision-coalescence among clusters themselves. The clusters can grow to a size at which they are regarded as particles, termed nascent particles.

Basically, as seen by the blue-colored route in Fig. 1, considering the above ultrafast nucleation processes, the morphologies of nanoparticle products are indeed determined by the competition between the time for particle-particle collisions governed by Brownian coagulation $\left(\tau_{\text {coll }}\right)$ and inter-particle 
coalescence, known as sintering in the ceramic community $\left(\tau_{\sin t}\right)$. If the characteristic collision time between particles is larger than the characteristic sintering time i.e. $\tau_{\text {coll }}>\tau_{\text {sint }}$, particles will coalesce before another collision event occurs, resulting in mono-disperse nanospheres. Meanwhile, if the collision time is less than the characteristic coalescence time, chained aggregates of nanoparticles will be formed. It is known that VAFS has been widely used for the industrial production of fumed silica, alumina, and pigmentary titania. However, the availability of volatile precursors at reasonable cost limits VAFS to only a few materials, such as single component $\mathrm{SiO}_{2}, \mathrm{Al}_{2} \mathrm{O}_{3}, \mathrm{TiO}_{2}, \mathrm{~V}_{2} \mathrm{O}_{5}, \mathrm{Fe}_{2} \mathrm{O}_{3}, \mathrm{ZrO}_{2}, \mathrm{SnO}_{2}$, their mixed composites $\mathrm{TiO}_{2} / \mathrm{SiO}_{2}, \mathrm{Al}_{2} \mathrm{O}_{3} / \mathrm{SiO}_{2}, \mathrm{~V}_{2} \mathrm{O}_{5} / \mathrm{TiO}_{2}, \mathrm{~V}_{2} \mathrm{O}_{5} / \mathrm{Al}_{2} \mathrm{O}_{3}, \mathrm{Cr} / \mathrm{TiO} 2, \mathrm{Fe} / \mathrm{TiO}_{2}, \mathrm{Zn} / \mathrm{TiO}$, $\mathrm{Pt} / \mathrm{TiO}_{2}, \mathrm{Cu} / \mathrm{ZnO} / \mathrm{Al}_{2} \mathrm{O}_{3}$, and even some non-oxide ceramic particles $\mathrm{TiN}, \mathrm{TiC}, \mathrm{TiB}_{2}, \mathrm{TiSi}_{2}, \mathrm{SiC}$, etc. $[1][2],[14]-[16],[22]-[28]$

In utilizing low-volatile and economical precursors such as aqueous metal salts (metal acetates or nitrates), liquid-fed aerosol flame synthesis (LAFS) is well suited. It was first proposed as early as 1977 by Sokolowaski et al. for the synthesis of $\mathrm{Al}_{2} \mathrm{O}_{3}$ nanoparticles by combusting droplets of aluminum acetylacetonate in benzene-ethanol, which is sprayed using an ultrasonic nozzle [29]. Since then, only two decades later, this liquid-fed flame synthesis method was further developed for nanoparticle syntheses of single- or multi-component metal oxides such as $\mathrm{Al}_{2} \mathrm{O}_{3}, \mathrm{TiO}_{2}, \mathrm{ZrO}_{2}, \mathrm{Y}_{3} \mathrm{Al}_{5} \mathrm{O}_{12}, \mathrm{Y}_{2} \mathrm{O}_{3} / \mathrm{ZrO}_{2}$ etc. by Laine and co-worker at University of Michigan (named as flame spray pyrolysis, FSP) [30]-[38] and other investigators at almost the same period [39][40]. Basically, if the liquid precursor solution drives the combustion process, with a dominated energy contribution (much larger than 50\%), the LAFS is usually called flame spray pyrolysis (FSP). Whereas, for energy contribution from the precursor solution that is lower, the LAFS process is commonly recognized as spray flame synthesis, which is also 
known as flame-assisted spray pyrolysis (FASP) in literature [5]. Since 2000, the overwhelming development of FSP technology, among all flame syntheses routes, can be attributed to Pratsinis and his collaborators Baiker, Mädler, Wegner, etc. at ETH [1][5][9],[26]-[28]. Instead of an ultrasonic nozzle, a pressured-assisted FSP, stabilized by six surrounding methane-oxygen premixed flamelets, was originally proposed in 2002 for the synthesis of single- or multi-component metal oxides nanomaterials [41]-[45]. Since then, the demonstrations of FSP or other LAFS processes have been successfully accomplished for the syntheses of materials based on dozens of elements in the periodic table, as also partially seen in recent reviews [5][26][28], which, in order of ascending atomic number, are given below.

$\mathbf{L i}\left(\mathrm{Li}-\mathrm{ZnO}, \mathrm{Li}-\mathrm{CuO}, \mathrm{LiMn}_{2} \mathrm{O}_{4}, \mathrm{LiCoO}_{2}, \mathrm{Li}_{4} \mathrm{Ti}_{5} \mathrm{O}_{12}, \mathrm{LiFe}_{5} \mathrm{PO}_{8}, \mathrm{Li}_{1.7} \mathrm{Al}_{0.3} \mathrm{Ti}_{1.7} \mathrm{Si}_{0.4} \mathrm{P}_{2.6} \mathrm{O}_{12}, \mathrm{Li}_{1.2} \mathrm{Mn}_{0.54} \mathrm{Ni}_{0.13} \mathrm{Co}_{0.13} \mathrm{O}_{2}\right.$, Li-doped $\left.\mathrm{Na}_{2} \mathrm{O} \cdot x \mathrm{Al}_{2} \mathrm{O}_{3}\right),{ }^{[46]-[52]}$

$\mathbf{N a}\left(\mathrm{Na}_{2} \mathrm{O}, \mathrm{NaCl}, \mathrm{Mn}-\mathrm{Na}_{2} \mathrm{WO}_{4} / \mathrm{SiO}_{2}\right.$, soda-lime glass, Co-soda lime glass, and species as before with $\left.\underline{\mathbf{L i}}\right),{ }^{[53]-[55]}$ $\mathrm{Mg}\left(\mathrm{MgO}, \mathrm{MgO} / \mathrm{Al}_{2} \mathrm{O}_{3}, \mathrm{MgO} / \mathrm{Fe}_{2} \mathrm{O}_{3}, \mathrm{Pd} / \mathrm{MgFe}_{2} \mathrm{O}_{4}, \mathrm{Ni}: \mathrm{MgO}-\mathrm{SiO}_{2}, \mathrm{Mg}-\mathrm{Ca}_{3}\left(\mathrm{PO}_{4}\right)_{2}, \mathrm{Mg}_{2} \mathrm{SiO}_{4}: \mathrm{Cr}, \mathrm{Cu}_{a} \mathrm{Mg}_{b} \mathrm{Al}_{c} \mathrm{O}_{x}\right.$, $\left.\operatorname{BaMgAl}_{10} \mathrm{O}_{17}: \mathrm{Eu}^{3+}\right),{ }^{[38],[56]-[62],[130]}$

Al $\left(\mathrm{Al}_{2} \mathrm{O}_{3}, \mathrm{Al}_{2} \mathrm{O}_{3} / \mathrm{SiO}_{2}, \mathrm{Al}_{2} \mathrm{O}_{3} / \mathrm{TiO}_{2}, \mathrm{Al}_{2} \mathrm{O}_{3} / \mathrm{V}_{2} \mathrm{O}_{5}, \mathrm{Al}_{2} \mathrm{O}_{3} / \mathrm{CoO}_{x}, \mathrm{Al}_{2} \mathrm{O}_{3} / \mathrm{NiO}, \mathrm{Al}_{2} \mathrm{O}_{3} / \mathrm{NiAl}_{2} \mathrm{O}_{4}, \mathrm{Al}_{2} \mathrm{O}_{3} / \mathrm{ZnO} \mathrm{Y}_{3} \mathrm{Al}_{5} \mathrm{O}_{12}\right.$, $Y_{3} \mathrm{Al}_{5} \mathrm{O}_{12}: \mathrm{Ce}^{3+}, \mathrm{Al}_{2} \mathrm{O}_{3} / \mathrm{ZrO}_{2}, \mathrm{Al}_{2} \mathrm{O}_{3} / \mathrm{CeO}_{x}, \mathrm{Al}_{2} \mathrm{O}_{3}: \mathrm{Ce}^{3+}, \mathrm{Al}_{2} \mathrm{O}_{3} / \mathrm{La}_{2} \mathrm{O}_{3}, \mathrm{Al}_{2} \mathrm{O}_{3}: \mathrm{Pr}^{3+}, \mathrm{Al}_{2} \mathrm{O}_{3} / \mathrm{Ce}_{x} \mathrm{Zr}_{\mathrm{l}-\mathrm{O}} \mathrm{O}_{2}, \mathrm{Al}_{2} \mathrm{O}_{3} / \mathrm{SiO}_{2} / \mathrm{TiO}_{2}$,

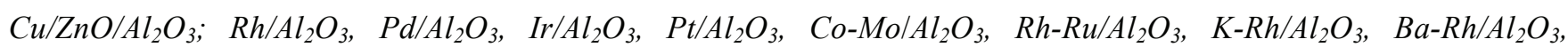
Pt- $\mathrm{K} / \mathrm{Al}_{2} \mathrm{O}_{3}, \mathrm{Pt}-\mathrm{Rh} / \mathrm{Al}_{2} \mathrm{O}_{3}, \mathrm{Pt}-\mathrm{Pd} / \mathrm{Al}_{2} \mathrm{O}_{3}, \mathrm{Pt}-\mathrm{Sn} / \mathrm{Al}_{2} \mathrm{O}_{3}, \mathrm{Pt}-\mathrm{Ba} / \mathrm{Al}_{2} \mathrm{O}_{3}, \mathrm{Pt}-\mathrm{Rh}-\mathrm{Ru} / \mathrm{Al}_{2} \mathrm{O}_{3}, \mathrm{Pd} / \mathrm{Al}_{2} \mathrm{O}_{3} / \mathrm{SiO}_{2}, \mathrm{Pd} / \mathrm{Al}_{2} \mathrm{O}_{3} / \mathrm{La}_{2} \mathrm{O}_{3}$, and species as before with $\underline{\mathrm{Mg}}),{ }^{[26],[32]-[38],[47],[58]-[75],[101][130][165][192][205][210][346][347][354][362][372]}$

Si $\left(\mathrm{SiO}_{2}, \mathrm{SiO}_{2} / \mathrm{TiO}_{2}, \mathrm{SiO}_{2} / \mathrm{V}_{2} \mathrm{O}_{5}, \mathrm{SiO}_{2} / \mathrm{Fe}_{2} \mathrm{O}_{3}, \quad \mathrm{SiO}_{2} / \mathrm{ZnO}, \mathrm{Zn}_{2} \mathrm{SiO}_{4}, \quad \mathrm{Y}_{2} \mathrm{SiO}_{5}, \quad \mathrm{SiO}_{2} / \mathrm{ZrO}_{2}, \quad \mathrm{SiO}_{2} / \mathrm{SnO}_{2}, \quad \mathrm{SiO}_{2} / \mathrm{TaO}_{2}\right.$, $\mathrm{SiO}_{2} / \mathrm{Yb}_{2} \mathrm{O}_{3} ; \mathrm{Ni} / \mathrm{SiO}_{2}, \mathrm{Cu} / \mathrm{SiO}_{2}, \mathrm{Ag} / \mathrm{SiO}_{2}, \mathrm{Ir} / \mathrm{SiO}_{2}, \mathrm{Au} / \mathrm{SiO}_{2}, \mathrm{Ag} / \mathrm{CeO}_{2}-\mathrm{SiO}_{2}, \mathrm{Au}-\mathrm{Ag} / \mathrm{SiO}_{2} ; \mathrm{Zn}_{2} \mathrm{SiO}_{4}: \mathrm{Mn}^{2+}, \mathrm{Y}_{2} \mathrm{SiO}_{5}: \mathrm{Eu}^{3+}$, 
$\mathrm{Y}_{2} \mathrm{SiO}_{5}: \mathrm{Eu}^{3+} / \mathrm{SiO}_{2}, \mathrm{SiO}_{2} / \mathrm{Ce}_{x} \mathrm{Zr}_{1-x} \mathrm{O}_{2}, \mathrm{Si}-\mathrm{WO}_{3}, \mathrm{SiO}_{2} / \mathrm{BiVO}_{4}, \mathrm{SiO}_{2}-\mathrm{TiO}_{2}-\mathrm{V}_{2} \mathrm{O}_{5}-\mathrm{WO}_{3}$, and species as before with $\underline{\mathrm{Na}}, \underline{\mathrm{Mg}}, \underline{\mathrm{Al}}$ ), [41][45][|47]|[55][57]|[62][65],[72][73],[75]-[90],,[189][327]|[347][373][463][465]|[476][495]

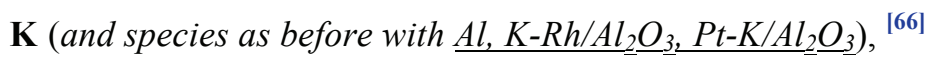

$\mathrm{Ca}\left(\mathrm{CaF}_{2}, \mathrm{CaO}, \mathrm{CaCO}_{3}, \mathrm{CaSO}_{4}, \mathrm{Ca}_{3}\left(\mathrm{PO}_{4}\right)_{2}, \mathrm{Mg}_{-} \mathrm{Ca}_{3}\left(\mathrm{PO}_{4}\right)_{2}, \mathrm{Zn}-\mathrm{Ca}_{3}\left(\mathrm{PO}_{4}\right)_{2}, \mathrm{Ca}_{10}\left(\mathrm{PO}_{4}\right)_{6}(\mathrm{OH})_{2-x} \mathrm{~F}_{x}\right),{ }^{[53][54],[91]-[95]}$

$\mathrm{Ti}\left(\mathrm{TiO}_{2}, \mathrm{~F}-\mathrm{TiO}_{2}, \mathrm{~V}-\mathrm{TiO}_{2}, \mathrm{TiO}_{2} / \mathrm{V}_{2} \mathrm{O}_{5}, \mathrm{Cr} / \mathrm{TiO} \mathrm{O}_{2}, \mathrm{Co} / \mathrm{TiO} \mathrm{O}_{2}, \mathrm{Mn} / \mathrm{TiO}_{2}, \mathrm{Fe}-\mathrm{TiO}_{2}, \mathrm{Fe} / \mathrm{TiO}\right.$, $\mathrm{Zn} / \mathrm{TiO} \mathrm{O}_{2}, \mathrm{Co} / \mathrm{TiO} \mathrm{O}_{2}, \mathrm{Ni} / \mathrm{TiO} \mathrm{O}_{2}$

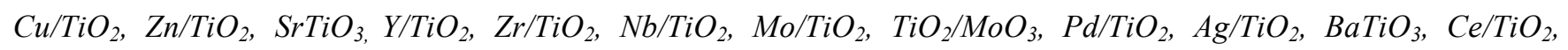
$\mathrm{TiO}_{2} / \mathrm{WO}_{3}, \mathrm{Pt} / \mathrm{TiO}_{2}, \mathrm{Pt}-\mathrm{Co} / \mathrm{TiO}_{2}, \mathrm{Pt}-\mathrm{Pd} / \mathrm{TiO} \mathrm{O}_{2}, \mathrm{Au} / \mathrm{TiO}_{2}, \mathrm{Au}-\mathrm{Ag} / \mathrm{TiO}_{2}, \mathrm{PbS} / \mathrm{TiO}_{2}, \mathrm{Ba}_{1-x} \mathrm{Sr}_{x} \mathrm{TiO}_{3}, \mathrm{SrTiO} \mathrm{O}_{3}: \mathrm{Pr}$, and species as before with $\underline{\mathrm{Li}}, \underline{\mathrm{Al}}, \underline{\mathrm{Si}}),{ }^{[47][51][62][78],[96]-[102],[104]]-[120],[324][350][351],[365]-[367],[463][474][4766][500]}$

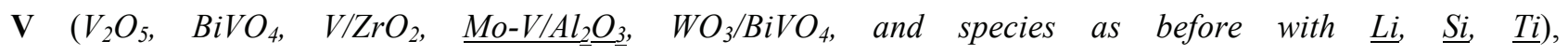
[28][63]|[76]|[77]|[78][90],,[100]-[102],[121]-[127],[364]

$\mathrm{Cr}\left(\mathrm{Cr}-\mathrm{WO}_{3}\right.$, Hastelloy, $\mathrm{Ni}-\mathrm{Cr}-\mathrm{Si}-\mathrm{B}$, and species as before with $\left.\underline{\mathrm{Mg}}\right),{ }^{[57][102][156]}$

Mn $\left(\mathrm{Mn}_{2} \mathrm{O}_{3}, \mathrm{ZnMn}_{2} \mathrm{O}_{4}, \mathrm{Mn} / \mathrm{ZnO}, \mathrm{MnO}_{x} / \mathrm{ZnO}, \mathrm{SrMO}_{3}, \mathrm{LaMnO}_{3 \pm \delta}, \mathrm{Pd} / \mathrm{LaMnO}_{3 \pm \delta}, \mathrm{Pd} / \mathrm{YMnO}_{3 \pm \delta}, \mathrm{La}_{1-x} \mathrm{Ag}_{x} \mathrm{MnO}_{3 \pm \delta}\right.$, $\mathrm{La}_{1-x} \mathrm{Ce}_{x} \mathrm{MnO}_{3}, \mathrm{La}(\mathrm{Mn}, \mathrm{Pd}) \mathrm{O}_{3}$, and species as before with $\left.\underline{\mathrm{Li}}, \underline{\mathrm{Ti}}\right),{ }^{[46][50][51][55][81][102],[129][-[135][358][359][468]}$

Fe $\left(\mathrm{Fe}_{2} \mathrm{O}_{3}, \mathrm{Fe}_{3} \mathrm{O}_{4}, \mathrm{FeO}, \mathrm{FePO}_{4}, \mathrm{Zn}-\mathrm{Fe}_{2} \mathrm{O}_{3}, \mathrm{Fe} / \mathrm{SnO} \mathrm{O}_{2}, \mathrm{Ag} / \mathrm{Fe}_{2} \mathrm{O}_{3} / \mathrm{Fe}_{3} \mathrm{O}_{4}, \mathrm{Au} / \mathrm{Fe}_{2} \mathrm{O}_{3} / \mathrm{Fe}_{3} \mathrm{O}_{4}, \mathrm{Au}-\mathrm{Ag} / \mathrm{Fe}_{2} \mathrm{O}_{3} / \mathrm{Fe}_{3} \mathrm{O}_{4}\right.$, $\mathrm{LaFeO}_{3 \pm \delta}, \mathrm{Pd} / \mathrm{LaFeO}_{3 \pm \delta}, \mathrm{Pd} / \mathrm{YFeO}_{3 \pm \delta}, \mathrm{Nd}: \mathrm{Co}: \mathrm{Fe}_{2} \mathrm{O}_{3}, \mathrm{Nd}-\mathrm{Fe}-\mathrm{B}$ alloy, and species as before with $\left.\underline{\mathrm{Li}}, \underline{\mathrm{Mg}}, \underline{\mathrm{Si}}, \underline{\mathrm{Ti}}\right)$, [51][79|[85]|[102][104][105][130],[136]-[145],[335]|[358|[373][469|[501]

$\mathrm{Co}\left(\mathrm{CoO}, \mathrm{Co}_{3} \mathrm{O}_{4}, \mathrm{Co}_{3} \mathrm{O}_{4}-\mathrm{NiO}, \mathrm{Co}_{3} \mathrm{O}_{4}-\mathrm{ZrO}_{2}, \mathrm{LaCoO}_{3}, \mathrm{Ru} / \mathrm{Co}_{3} \mathrm{O}_{4}-\mathrm{ZrO}_{2}, \mathrm{Pd} / \mathrm{LaCoO}_{3 \pm \delta}, x \mathrm{Ag} / \mathrm{LaCoO} \mathrm{Ca}_{1-x} \mathrm{Ag}_{x} \mathrm{CoO}_{3}\right.$, $L a_{1-x} \mathrm{Ce}_{x} \mathrm{CoO}_{3+\delta}, \quad \mathrm{La}_{1-x} \mathrm{Eu}_{x} \mathrm{CoO}_{3+\delta}, \quad$ and species as before with $\left.\underline{\mathrm{Na}}, \quad \underline{\mathrm{Al}}, \quad \underline{\mathrm{Fe}}, \quad \underline{\mathrm{Ti}}\right), \quad[46][48 \mid[86][102][103]$ $[130][140][141],[146]-[153],[160][210][351][360][361]$

Ni $\left(\mathrm{NiO}, \mathrm{NiO}-\mathrm{CO}_{3} \mathrm{O}_{4}, \mathrm{NiO}-\mathrm{CuO}, \mathrm{Ni}-\mathrm{Zn}\right.$ alloy, $\mathrm{Ni} / \mathrm{ZrO}$, $\mathrm{NiO}-\mathrm{MoO}_{3}$, $\mathrm{Ni}-\mathrm{Mo}$ alloy, $\mathrm{La}_{2} \mathrm{NiO}_{4 \pm \delta}$, and species as before with $\underline{M g}, \underline{A l}, \underline{T i}, \underline{C r}, \underline{C o}),{ }^{[46][56][64][87][102][133],[153]-[156],[160]}$ 
$\mathrm{Cu}\left(\mathrm{CuO}, \mathrm{ZnO} / \mathrm{CuO}, \mathrm{ZnWO}_{4} / \mathrm{CuO}, \mathrm{MoO}_{2} / \mathrm{CuO}, \mathrm{Cu}-\mathrm{ZrO}_{2}, \mathrm{Cu}-\mathrm{CeO}_{2}, \mathrm{SnO} 2-\mathrm{CuO}, \mathrm{WO}_{3} / \mathrm{CuO}, \mathrm{Cu}-\mathrm{Ce}-\mathrm{Zr}, \mathrm{Cu}-\mathrm{Zr}-\mathrm{Al}\right.$, and species as before with $\underline{\mathrm{Mg}}, \underline{\mathrm{Si}}, \underline{\mathrm{Al}}, \underline{\mathrm{Ti}}, \underline{\mathrm{Ni}}),{ }^{[49][60][61][86][102][109][153],[157]-[168][367]}$

Zn (ZnO, Ga-ZnO, Nb-ZnO, Ru-ZnO, Pd-ZnO, Ag-ZnO, In-ZnO, Sn-ZnO, Au-ZnO, Pt-ZnO, W-ZnO, and species as before with $\underline{\mathrm{Li}}, \underline{\mathrm{Si}}, \underline{\mathrm{Ca}}, \underline{\mathrm{Mn}}),{ }^{[44][45][[52][80][81][106][131][132][142][154],[169]-[174],[206][233][338] ~[427],[466]-[469] ~}$

$\mathbf{G a}\left(\mathrm{Ga}_{2} \mathrm{O}_{3}, \mathrm{Cu}(\mathrm{In}, \mathrm{Ga}) \mathrm{O}_{2}, \mathrm{Eu}-\mathrm{Ga}_{2} \mathrm{O}_{3}\right.$, and species as before doped with $\left.\mathrm{Zn}, \mathrm{Ga}-\mathrm{ZnO}\right),{ }^{[161][174][176][338]}$

$\mathbf{G e}\left(G e O_{x}\right){ }^{[177]}$

$\mathrm{Sr}\left(\mathrm{SrF}_{2}, \mathrm{SrTiO}_{3}: \mathrm{Pr}, \mathrm{Sr}_{5}(\mathrm{PO} 4)_{3} \mathrm{Cl}: \mathrm{Eu}^{2+}\right.$, and species as before with $\left.\underline{\mathrm{Mn}}\right),{ }^{[33][107][108][133],[178]-[180]}$

$\mathbf{Y}\left(\mathrm{Y}_{2} \mathrm{O}_{3} / \mathrm{ZrO}_{2}, \mathrm{Y}_{2} \mathrm{O}_{3}: \mathrm{Eu}^{3+}, \underline{\mathrm{Y}_{2}} \underline{\mathrm{SiO}} \underline{\underline{5}} \underline{\underline{\mathrm{Eu}}}{ }^{3+}\right.$, and species as before with $\left.\underline{\mathrm{Si}}, \underline{\mathrm{Al}}\right),{ }^{[34][88][102],[181]-[196][372]}$

$\mathrm{Zr} \quad\left(\mathrm{ZrO}_{2}, \quad \mathrm{ZrO}_{2} / \mathrm{La}_{2} \mathrm{O}_{3}, \quad \mathrm{ZrO}_{2} / \mathrm{CeO}_{2}, \quad \mathrm{Ce}_{x} \mathrm{Zr}_{1-x} \mathrm{O}_{2}, \quad \mathrm{Al}_{2} \mathrm{O}_{3} / \mathrm{Ce}_{x} \mathrm{Zr}_{1-x} \mathrm{O}_{2}, \quad \mathrm{Rh} / \mathrm{Ce}_{x} \mathrm{Zr}_{1-x} \mathrm{O}_{2}, \quad \mathrm{Pt} / \mathrm{Ce}_{x} \mathrm{Zr}_{1-x} \mathrm{O}_{2}\right.$, $\mathrm{Pt}-\mathrm{Ba} / \mathrm{Ce}_{x} \mathrm{Zr}_{1-x} \mathrm{O}_{2}$, and species as before with $\left.\underline{\mathrm{Si}}, \underline{\mathrm{Al}}, \underline{\mathrm{Co}}, \underline{\mathrm{Al}}\right),{ }^{[62][75][83][87][101][102][125][152][162][165],[194]-[205],[328][329][361][362][481]}$ Nb $(\mathrm{Nb}$-species as before with $\underline{\mathrm{Ti}}, \underline{\mathrm{Zn}}),{ }^{[108]-[110],[172][206]}$

Mo $\left(\mathrm{MoO}_{3}, \quad \alpha-\mathrm{Bi}_{2} \mathrm{Mo}_{3} \mathrm{O}_{12}, \quad \gamma-\mathrm{Bi}_{2} \mathrm{MoO}_{6}, \quad \beta-\mathrm{Bi}_{2} \mathrm{Mo}_{2} \mathrm{O}_{9}, \quad\right.$ and species as before with $\left.\underline{\mathrm{V}} \underline{\mathrm{Ni}}, \underline{\mathrm{Cu}}\right)$, [65] [102][126][153][155][159],[207]-[211]

$\mathbf{R u}\left(\mathrm{Ru} / \mathrm{SnO}_{2}\right.$, species as before doped with $\left.\underline{\mathrm{Al}}, \underline{\mathrm{Co}}, \underline{\mathrm{Zn}}, \underline{\mathrm{Zr}}\right),{ }^{[68][152][206][212]}$

Rh (species as before doped with $\underline{M g}, \underline{A l}, \underline{\mathrm{Zr}}, \underline{\mathrm{Co}}, \underline{\mathrm{Ce}}),{ }^{[59][66][68][204][205]}$

Pd $\left(\mathrm{Pd} / \mathrm{CeO}_{2}, \quad \mathrm{Pd} / \mathrm{SnO}_{2}, \quad \mathrm{Pd}-\mathrm{WO}_{3}, \quad\right.$ and species as before doped with $\left.\underline{\mathrm{Mg}}, \underline{\mathrm{Al}}, \quad \underline{\mathrm{Si}}, \underline{\mathrm{Ti}}, \quad \underline{\mathrm{Zr}}, \quad \underline{\mathrm{Co}}\right)$, [59|[62][68][69][71][73][112][134][149][150][171],[213]-[215],[324][346][358][366][466]

Ag (species as before doped with $\underline{\mathrm{Si}}, \underline{\mathrm{Ca}}, \underline{\mathrm{Ti}}, \underline{\mathrm{Mn}}, \underline{\mathrm{Fe}}, \underline{\mathrm{Zn}}),{ }^{[85][89][92][135][151][169]}$

In $\left(\mathrm{In}_{2} \mathrm{O}_{3}, \mathrm{In}_{2} \mathrm{O}_{3} / \mathrm{SnO}_{2}, \mathrm{In}_{4} \mathrm{Sn}_{3} \mathrm{O}_{12}, \mathrm{In}_{1.9} \mathrm{Sn}_{0.1} \mathrm{O}_{3}\right.$ (ITO), and species as before doped with $\left.\underline{\mathrm{Ti}}, \underline{\mathrm{Zn}}, \underline{\mathrm{Cu}}, \underline{\mathrm{Ga}}\right)$, [52][161][173][174][216][217]

Sn $\left(\mathrm{SnO}_{2}, \quad \mathrm{Pd} / \mathrm{SnO} \mathrm{O}_{2}, \quad \mathrm{Sb} / \mathrm{SnO}_{2}, \quad \mathrm{Pt}-\mathrm{SnO} \mathrm{O}_{2}, \quad \mathrm{Pt} / \mathrm{SnO} \mathrm{O}_{2}, \quad\right.$ and species as before doped with $\left.\underline{\mathrm{Si}}, \underline{\mathrm{Zn}}\right)$, 
$[52][82][115][143][166][206],[212]-[224],[354][427][476]$

Sb (species as before doped with $\left.\underline{\mathrm{Sn}, \mathrm{Sb} / \mathrm{Sn} \mathrm{O}_{2}}\right),{ }^{[215]}$

$\mathbf{B a}\left(\mathrm{BaF}_{2}, \mathrm{Ho}-\mathrm{BaF}_{2}, \mathrm{BaCO}_{3}, \mathrm{Pt}-\mathrm{BaCO}, \mathrm{Pt}-\mathrm{Ba} / \mathrm{CeO}_{2}\right.$, and species as before doped with $\left.\underline{\mathrm{Al}}, \underline{\mathrm{Zr}}\right),{ }^{[53][66][67][107][116][203]}$ [225][226]

$\mathbf{L a}\left(\mathrm{La}_{2} \mathrm{O}_{3}\right.$, species as before doped with $\left.\underline{\mathrm{Al}}, \underline{\mathrm{Co}}, \underline{\mathrm{Zr}}\right),{ }^{[71][108][134][135],[146]-[151],[193],[358]-[360]}$

$\mathrm{Ce}\left(\mathrm{CeO}_{2}, \mathrm{Ce}_{1-x} G d_{x} \mathrm{O}_{2-x / 2}, \mathrm{Bi} / \mathrm{CeO}_{2}\right.$, and species as before with $\underline{\mathrm{Ti}}, \underline{\mathrm{Si}}, \underline{\mathrm{Al}}, \underline{\mathrm{Zr}}, \underline{\mathrm{Bi}}$ (also $\left.\underline{\mathrm{Rh}}, \underline{\mathrm{Pt}}, \underline{\mathrm{Ba}}\right)$ ), $[12][42][74][75][89][101][102][117][144],[162]-[165],[192],[201]-[205],[226],[228]-[230],[359][360][372]$

$\operatorname{Pr}$ (species as before with $\underline{\mathrm{Al}, \mathrm{Al}_{2}} \underline{\underline{O_{3}}}: \underline{\operatorname{Pr}} ; \underline{\operatorname{Sr}, \operatorname{SrTiO}} \underline{3}: \underline{\operatorname{Pr}}$ ), ${ }^{[74][178][179]}$

Nd (species as before with $\left.\underline{\mathrm{Fe}}, \underline{\mathrm{Nd}: \mathrm{Co}: \mathrm{Fe}_{2}} \underline{\mathrm{O}_{3}}\right),{ }^{[141][145]}$

$\mathbf{E u}\left(\mathrm{Eu}: \mathrm{Gd}_{2} \mathrm{O}_{3}, \mathrm{Eu}: \mathrm{Gd}_{2} \mathrm{O}_{3} / \mathrm{Nd}_{2} \mathrm{Co}: \mathrm{Fe}_{2} \mathrm{O}_{3}\right.$, as seen before $\left.\underline{\mathrm{Y}}_{2} \underline{\mathrm{O}}_{3}: \mathrm{Eu}^{3+}, \underline{\mathrm{Y}_{2}} \underline{\mathrm{SiO}_{5}}: \underline{\mathrm{Eu}}{ }^{3+}\right),{ }^{[88][180],[182]-[190],[231][338][360]}$

Gd (species as before with $\underline{\text { Eu}}),{ }^{[141][193],[229]-[231]}$

Tb (species as before with $\underline{Y}),{ }^{[190][191]}$

Ho (species as before with $\underline{B a}),{ }^{[53]}$

$\mathbf{E r}($ species as before with $\underline{Y}),{ }^{[188][193]}$

$\mathbf{Y b}$ (species as before with $\underline{\mathrm{Si}}),{ }^{[193]}$

Ta (species as before doped with $\left.\underline{\mathrm{Si}}, \underline{\mathrm{SiO}} \underline{\underline{2}} \underline{\mathrm{Ta}_{2}} \underline{\mathrm{O}_{5}}\right),{ }^{[84]}$

$\mathbf{W}\left(\mathrm{WO}_{3}, \mathrm{Au}-\mathrm{WO}_{3}, \quad\right.$ and species as before doped with $\left.\underline{\mathrm{Si}-T i-V}, \underline{\mathrm{Cr}}, \underline{\mathrm{Cu}}, \underline{\mathrm{Zn}}, \underline{W}\right)$, $[55][78][118][128][167][168][206][232],[234]-[237],[465][475][619]$

Ir (species as before doped with $\underline{\mathrm{Al}}, \underline{\mathrm{Si}}),{ }^{[72]}$

Pt $\left(\mathrm{Pt} / \mathrm{WO}_{3}\right.$, and species as before doped with $\left.\underline{M g}, \underline{A l}, \underline{T i}, \underline{Z n}, \underline{Z r}, \underline{B a}, \underline{S n}\right)$, [58][59],[67]-[70], [97][113][114][119][167][170][206],[222]-[226],[351][354][366][475][477] 
Au (Au-soda lime glass, and species as before doped with $\underline{\mathrm{Mg}} \underline{\mathrm{Al}}, \underline{\mathrm{Si}}, \underline{\mathrm{Ti}}, \underline{\mathrm{Fe}}, \mathrm{Cu}),{ }^{[85][170][350][365]}$

$\mathbf{P b}$ (species as before doped with $\left.\underline{\mathrm{Ti}}, \underline{\mathrm{PbS} / \mathrm{Ti} \mathrm{O}_{2}}\right),{ }^{[120]}$

$\mathbf{B i}\left(\mathrm{Bi}_{1} \mathrm{Bi}_{2} \mathrm{O}_{3}\right.$, and species as before with $\underline{\mathrm{Si}, \mathrm{V}, \mathrm{Mo}, \mathrm{Ce})} .{ }^{[43][90 \mid[123][124][211][228][238][239]}$

Appendix Table 1 further presents the properties of metal precursors and, particularly, their demonstrated solvents for the LAFS process, including those mentioned above. Appendix Table 2 lists solvents (and their properties) that are used for liquid-fed aerosol flame syntheses (LAFS). In the LAFS process such as FSP or FASP, the governing mechanism of particle formation is a combination of the aforementioned gas-to-particle conversion with intraparticle reaction (or namely spray processes), as given in the red route in Fig. 1. The ratio of the solvent-fuel boiling temperature to the precursor decomposition temperature, i.e. $T_{b p(\text { solvent })} / T_{d / m p(\text { precursor })}$, plays an important role in determining particle formation routes. For high boiling point solvents, i.e. $\left(T_{b p} / T_{d / m p}>1.05\right)$, the complete evaporation of the metal-precursor, the decomposition/combustion of the precursor, and the subsequent nucleation/coagulation of the desired species from the gas phase results in the formation of ultrafine oxide nanoparticles, which is also known as gas-to-particle conversion, with the same fundamental mechanism as that in VAFS (blue route in Fig. 1). For low boiling point solvents, i.e. $\left(T_{b p} / T_{d / m p}<1.05\right)$, the solvent evaporates; and the precursor precipitates on the droplet surface (red route), forming an outer shell that can trap the remaining solvent to be evaporated inside the shell, which eventually results in hollow, shell-like, or inhomogeneous oxide particles at much larger micron-sizes [240]. Such kind of inhomogeneous particle formation via intraparticle reaction is also generally known as droplet-to-particle conversion. Moreover, in the case of $T_{b p} / T_{d / m p}<1.05$, both inhomogeneous micron-particles due to droplet-to-particle conversion and homogenous nanoparticles due to 
gas-to-particle conversion can co-exist, as schematically illustrated in Fig. 1 [5]. The interchange processes (colored as green routes) between these two kinds of conversion processes may occur, which further include the heterogeneous nucleation of product vapor on large particles from droplet-to-particle conversion and the scavenging of nascent particles (from gas-to-particle conversion) by these large particles.

Apart from $T_{b p(\text { solvent })} / T_{d / m p(\text { precursor) })}$, the second criterion is the combustion specific enthalpy, as shown in Appendix Table 2. In the case when flame synthesis is operated at high combustion specific enthalpy ( $>4.7 \mathrm{~kJ} / \mathrm{g}_{\mathrm{gas}}$ ), sufficient heat is provided to evaporate the precursor to form homogeneous solid fine particles, even at $T_{b p} / T_{d / m p}<1$. These two criteria are experimentally evidenced by the FSP-made products, such as $\mathrm{MgO}, \mathrm{Al}_{2} \mathrm{O}_{3}, \mathrm{SiO}_{2}, \mathrm{Fe}_{2} \mathrm{O}_{3}, \mathrm{Co}_{3} \mathrm{O}_{4}, \mathrm{ZnO}, \mathrm{Y}_{2} \mathrm{O}_{3}, \mathrm{CeO}_{2}, \mathrm{Bi}_{2} \mathrm{O}_{3}$, or $\mathrm{MgAl}_{2} \mathrm{O}_{4}$ [240][241].

According to the properties of solvents and metal precursors listed in Appendix Tables 1-2, most metal alkoxides (except aluminum propoxide), in contrast to other precursors such as nitrates, can easily meet the criterion of $T_{b p} / T_{d / m p}>1.05$, enabling the synthesis of dense and homogenous ultrafine nanoparticles. Therefore, the alkoxide precursors, mainly metal methoxides or pentoxides, are usually used in FSP applications because of their high volatilities, high combustion enthalpies, miscibility in organic solvents, and low viscosities [5]. Metal carbonxylates are another possible choice for FSP. Recently, inexpensive, low-volatile metal nitrate precursors for $\mathrm{Mg}$, $\mathrm{Al}, \mathrm{Fe}$, Co and $\mathrm{Zn}$ have been investigated [241]. The influence of solvent parameters such as boiling point, combustion enthalpy, and chemical reactivity on the formation of either homogeneous nanoparticles by gas-to-particle conversion or large particles through droplet-to-particle conversion was systematically examined. The volatile metal carboxylates, formed by mixing carboxylic acid (e.g., 2-ethylhexanoic acid, EHA, or propionic 
acid) with metal nitrate and solvent (e.g. ethanol), has been found to significantly enhance the production of homogeneous nanoparticles over large inhomogeneous particles. In contrast, the incremental increase of solvent boiling point and combustion enthalpy only slightly favor the formation of homogeneous nanoparticles. The carboxylic-acid-reacted metal nitrate precursors have been demonstrated for oxide nanoparticles of $\mathrm{Mg}, \mathrm{Al}, \mathrm{Fe}, \mathrm{Co}, \mathrm{Zn}, \mathrm{Bi}$, etc. [43][241]. Besides metal nitrates, the reactions of EHA with other low-cost acetates $(\mathrm{Na}, \mathrm{Ba}, \mathrm{Sr}, \mathrm{Bi})$, oxides $(\mathrm{Ca}, \mathrm{Mg}, \mathrm{Ho})$, hydroxides $(\mathrm{Ca}$, $\mathrm{Al})$, and even carbonates $(\mathrm{Na}, \mathrm{Zr})$ had also been shown to enhance the gas-to-particle conversion for homogeneous nanoparticle formation, as summarized in Ref. [5].

\subsection{Scope of the Review}

Generally, provided that the suitable organometallic or water-soluble precursors are available, LAFS overcomes the limitation of VAFS of producing only a few kinds of metal-oxides, and opens almost the whole periodic table for possible material compositions. Systematic experiments have been conducted by key groups including Pratsinis, Mädler (now at Bremen), and Baiker from ETH, Laine from Michigan, their collaborators and other researchers around the world since the late 90s and early 2000s [5][26][28][242]. Thus, given the robustness of flame aerosol synthesis, a question arises: what is the next big challenge for it, as well as its future prospects? The answer may lie in two extremes. One direction is upwardly, i.e. the scale-up and industrialization of the flame aerosol reactor to achieve scalable and economical production of functional nanomaterials. The other direction is downwardly, i.e. going small and zooming in to clarify the underlying physicochemical mechanisms that governs the

process, and then based on them, to exquisitely fabricate and manipulate the as-synthesized 
nanoparticles with tailored properties toward a functional device at nanoscale.

To face the future challenges at the two extremes, we should recognize that flame synthesis is an interdisciplinary research field between materials science and combustion science [22]. This new field should benefit from both disciplines as well as contribute to them in return. Generally, materials science is focused on characterizing solid-state products, including nanomaterials with new properties and devices with improved functional performances. The huge progress on flame synthesis methods, i.e. VAFS, FSP, and FASP, within the past three decades benefits tremendously from the rapid development of materials science. However, most combustion scientists still consider particle formation more from the viewpoint of pollutant (e.g. soot, minerals) formation, despite the ostensible interest in the synthesis aspect manifested by persistent discussions of several groups at Combustion Symposia since the early 1990's [243]-[249], as well as the advocacy for it in two key review papers [16][22]. Apart from the Combustion Symposia, the interaction of investigators from both materials and combustion sides may be found in the recent MRS symposia on flame synthesis of nanomaterials established since 2012. With such mixing/merging of expertise across various disciplines, fundamental understanding of controlling flame synthesis processes at either atomic or macroscopic levels, rather than empirical adjusting of parameters in established synthesis methods, is requisite to propel the field forward. For instance, new chemical kinetic mechanisms (e.g. based on ab initio quantum chemistry and reaction rate laws), advanced aerosol dynamics modeling (from population balance models, classic molecular dynamics, and Monte Carlo simulations, etc.), and their integration with detailed computational fluid dynamics (gas phase transport), along with new in situ diagnostic tools and novel flame aerosol reactor configurations, are providing better understanding of fundamental processes and improving flame synthesis capabilities, 
yields, and materials. These aspects establish the scope of this review, as motivated by six key research needs/challenges:

- (1) Need for controlling sizes or tailoring nanostructures with atomic precision;

- (2) Need for scalable and economical manufacturing of nanostructured materials;

- (3) Need for doped flame synthesis techniques to produce functional nanocomposites, not only composites of mixed metal oxides, but those of carbon-metal oxides as well;

- (4) Need for reliable fabrication of flame-made materials into functional devices;

- (5) Need for building theoretical modeling and computational simulations across several scales;

- (6) Need for developing in situ diagnostics for gas, liquid, and solid phases in synthesis flow fields.

Addressing these exigent needs, this paper is organized into the following sections. Section 2 summarizes the development of both flame aerosol reactors and spraying techniques that may partially answer Needs (1) or (2). With respect to Needs from (2) to (6), respectively, Section 3 briefly reviews the progress on synthesis of doped nanomaterials with various typical nanostructures achieving different functions; Section 4 presents the assembly of nanostructured materials into thin films and the further fabrication of functional devices; Section 5 summarizes progress on theoretical approaches across several orders of scales in flame synthesis processes; and Section 6 summarizes in situ diagnostics. Sections 5 and 6 have been less emphasized in previous works, as compared to the tremendous number of publications on synthesis methods. Moreover, after the last two review papers on flame synthesis of metal oxides appeared in PECS in 1998 [1][2], there are lots of excited progresses/achievements in fields of doped synthesis nanocomposite, fabrication of functional devices and, particularly, in the 
aspects of modeling/diagnostic technologies, which are also two main focus in this review.

\section{Development of flame aerosol reactors: the trends from "free-jet" to stagnation configurations} and from vapor-fed to liquid-fed ones

The historical development of flame aerosol reactors, namely burner types, demonstrates how industrial practice can be ahead of laboratory research. For example, since 1943, a commercial "Aerosil Process", capable of producing nanopowders of fumed $\mathrm{SiO}_{2}, \mathrm{Al}_{2} \mathrm{O}_{3}$, and $\mathrm{TiO}_{2}$, used VAFS to perform $\mathrm{SiCl}_{4}$ hydrolysis in an oxy-hydrogen flame ambience by employing a multi-annulus, co-flow diffusion flame burner [250]. Similarly, in the 1970s, the commercial "furnace black" process used LAFS by employing a modern "jet-type" spray flame burner under fuel-rich conditions, which can be viewed as an origin of flame spray pyrolysis [251]. Since the pioneering work of Dr. Ulrich in the 1970s, several surveys have been reviewed on the evolution of burner types for flame synthesis. For example, two comprehensive reviews by Pratsinis and by Wooldridge, both published in 1998 in PECS, presented useful summaries of such studies prior to 1997 [1][2]. In 2005, Rosner reviewed three basic types of VAFS burners, i.e., premixed flat flame, multi-annulus coflow diffusion flame, and counterflow diffusion flame, along with one "spray" diffusion flame (LAFS burners), which were employed for most laboratory studies of flames synthesis [252]. Then in 2006, Roth presented an invited plenary of the $31^{\text {st }}$ International Combustion Symposium, reviewing four basic types of VAFS burners, i.e., burner-stabilized flat flame, stagnation-point premixed flame, single or multi-annulus coflow diffusion flame, and counterflow diffusion flame, along with FSP for LAFS [22]. Considering a recent overview on burner types for the synthesis of carbon-based nanomaterials [17], this section is focused on synthesis 
of metal oxide compounds.

Fig. 2 illustrates basic burner types for both vapor-fed and liquid-fed aerosol flame synthesis, in accordance with both historical progression and flame classification. The first three, i.e. from (a) to (c), are "free-jet" flame burners, which are operated, respectively, in the following modes: (a) multi-annulus diffusion flames, (b) combination of inner single diffusion flame plus outer premixed flat flame (known as McKenna burner); (c) combination of inner single diffusion flame plus outer multi-element diffusion flat flames (Hencken burner); (d) counterflow diffusion flame; and (e) flame spray pyrolysis. The last five from (f.1-4) and (g) are all modified stagnation-point configurations where flow from the nozzles of burners impinge on temperature-controlled substrates. It must be noted that, because of the importance of molecular mixedness, combustion behaves quite differently depending on whether the reactants, fuel and oxidizer, are initially pre-mixed or not. For premixed flames, the reactants are already well-mixed before reaction, whereas for diffusion flames, the reactants are initially separated and are brought together through mixing by either/both molecular diffusion and bulk advection. Table 1 summarizes the characteristics of various typical burners that have been employed in flame aerosol synthesis. As far as the decomposition of precursor is concerned, in contrast to non-premixed diffusion flame, the premixed flame mode of some burners may favor the low-temperature abstraction initiation $\left(\mathrm{RH}+\mathrm{O}_{2} \rightarrow \mathrm{R}+\mathrm{HO}_{2}\right)$ of precursor, besides the high-temperature thermal decomposition $(\mathrm{RH}+\mathrm{M} \rightarrow \mathrm{R}+\mathrm{H}+\mathrm{M})$. Importantly, for most precursors, the particle formation and growth in different burners is significantly affected by the temperature-time profile (i.e., maximum temperature, quenching rate, and residence time, etc.) [253]. For these precursors that are sensitive to the radical species, the radical concentrations in the different reactors will also affect the particle, either by the radical driven precursor decomposition or the 
quenching of radical species on the particle surface as flame inhibitor [254].

\subsection{Coflow diffusion flame burner}

The coflow diffusion flame burner, which possesses advantages of offering high flexibility by broad operational windows and safety without flash-back, has been widely employed for commercial production of flame-made nanomaterials such as the so-called "chloride process", "Aerosil process" (Degussa), and, more recently, "sodium flame and encapsulation (SFE) process" (Cabot Corporation) [247][250][255]. Other than a few applications of single jet flames, the coaxial multi-annulus configurations, consisting of three concentric stainless-steel tubes, are commonly utilized so that the gaseous fuel and oxidizer streams can be separated from the central stream that contains the metal precursor, as shown in Fig. 2(a). In early 1972, Formenti et al. [15] introduced the setup for lab-scale investigation on the synthesis of metal chlorides in $\mathrm{H}_{2} / \mathrm{O}_{2} / \mathrm{N}_{2}$ diffusion flames to produce $\mathrm{TiO}_{2}, \mathrm{SiO}_{2}$, $\mathrm{Al}_{2} \mathrm{O}_{3}, \mathrm{ZrO}_{2}, \mathrm{Fe}_{2} \mathrm{O}_{3}$, and $\mathrm{GeO}_{2}$ nanopowders. The classic mixing configuration is arranged where oxidizer stream in the outermost tube, inert-gas-carried precursor in the inner tube, and fuel stream in the middle tube. The inverse mixing configuration, with methane flowing through the outermost tube and air flowing through the inner tube, as shown in Fig. 2(a), was studied to compare with the classic mixing one, demonstrating good potential for producing much finer particles. With air-flow mixed with precursor in the inner tube, the pre-dilution of metal precursor by air flow before its introduction to the flame front is a dominating factor for depressing particle growths, resulting in reduced size [255]. As

presented in Table 1, the inverse mixing burner produces NPs with size of $11 \mathrm{~nm}$ while the regular mixing one produces those of $105 \mathrm{~nm}$, under the identical yield of $3.7 \mathrm{~g}$ per MJ heat input from the 
methane. With respect to the diameter of outer tube, the product $\mathrm{TiO}_{2}$ flux is about $920 \mathrm{~g} / \mathrm{min} \cdot \mathrm{m}^{2}$.

However, a significant disadvantage of the coflow diffusion flame reactor is that its high-temperature post-flame zone can be sufficiently long causing uncontrollable particle growth. The temperature quenching rate in the post-flame zone (indicatively after the position of maximum temperature) has been measured to be $\sim 70 \mathrm{~K} / \mathrm{cm}$ (and mostly less than $100 \mathrm{~K} / \mathrm{cm}$ ) for the co-flow diffusion burner [256]. As a result, in such 'long hot flames,' spherical particles with a low degree of agglomeration usually have primary particle diameters of 50-100 $\mathrm{nm}$ and even above $200 \mathrm{~nm}$, greatly curbing their functional use as flame-made nanomaterials [255][257].

Many methods have been attempted to control particle growth, e.g. sintering and agglomeration of particles. For instance, in the late 90's, the control of agglomeration was achieved with sodium flames by Dufaux and Axelbaum [247], and with low-pressure flames by Glumac et al [258]. However, as for the coflow flame burner that is focused in this section, it is still difficult to attain nonagglomerate spherical particles with much smaller sizes (e.g., less than $50 \mathrm{~nm}$ ). The burner outlet velocity difference between the fuel and oxygen jets, $\Delta v=v_{o x}-v_{f u e l}$, has been identified as an effective parameter to control the sizes of primary particles in agglomerates down to $20 \mathrm{~nm}$, when $\Delta v$ is larger than $10 \mathrm{~m} / \mathrm{s}$. The reason is that, for larger $\Delta v$, increased entrainment of ambient air from higher oxygen outlet velocity shortens and cools the flame, leading to slower sintering and thus smaller primary particle diameters [256][257]. For instance, as the yield of NPs increases from 4.0 to $20 \mathrm{~g} / \mathrm{MJ}$-fuel (i.e. 5 to $25 \mathrm{~g} / \mathrm{h} \mathrm{SiO}_{2}$ ), the size of primary particle enlarges from 13 to $27 \mathrm{~nm}$ at the condition of $\Delta v=20 \mathrm{~m} / \mathrm{s}$, exhibiting a scaling relationship of $Y d_{p}^{2.2}$. The order of 2.2, which is less than 3, implies a certain degree of aggmoleration of primary nanoparticles. Furthermore, the increase in average primary particle diameter $\left(d_{p}\right)$ with 
increasing yield of $\mathrm{SiO}_{2}$ could be explained by the increase of the flame enthalpy content, resulting in

higher flame temperatures and therefore higher sintering rates.

However, despite the attainment of smaller primary particles, the formed materials are nonspherical with high degree of agglomeration. Accordingly, Wegner and Pratsinis developed a nozzle-quenching process, in which a critical flow nozzle is placed at the desired position at downstream of the particle-laden flame, to achieve controlled flame synthesis of metal oxides [259]. Using this flame-nozzle process, nonagglomerated, spherical titania particles are created, with primary particle sizes precisely controlled from $5 \mathrm{~nm}$ to $60 \mathrm{~nm}$ by selecting the burner-nozzle distance. This quenching procedure rapidly slows down particle growth downstream of the nozzle, thereby "freezing" the particle size and the morphology of the upstream aerosol. Furthermore, by introducing and stabilizing oxygen vacancies in the titania particles with the quenching procedure, the phase composition of the product powder can be independently controlled from $85 \mathrm{wt}$ \% to 5 wt. $\%$ anatase with the balance rutile. This broad operational window with respect to product particle size, morphology, crystallinity, and agglomeration shows the high potential of the flame-nozzle process to be successfully applied to other flame configurations for the cost-effective syntheses of silica, alumina, and other oxide and nonoxide ceramics, as well as carbon black and metals like nickel and iron [259][260].

\subsection{McKenna flat-flame burner and its supported system}

The McKenna burner has been widely used for many years to produce flat premixed flames, which is assumed to be one-dimensional and considered as a standard, at least under lean and close-to stoichiometric conditions [261]. The burner had been employed in many laboratories around the world 
for the development and calibration of optical diagnostic techniques [262]-[266]. The McKenna burner was recently proposed and utilized as a standard burner for the study of the mechanisms responsible for soot formation, and in particular, for the development of in situ soot diagnostics using the laser-induced incandescence (LII) technique [266]-[269].

The McKenna flat-flame burner, operating with a laminar premixed $\mathrm{CH}_{4} / \mathrm{N}_{2} / \mathrm{O}_{2}$ mixture, has the advantage of a simple geometry. Nevertheless, it has served as a model for more complicated industrial processes, such as the Cab-O-Sil process, which is based on premixed hydrocarbon flame synthesis (Ulrich, 1971 [14]). Originally, Ulrich and his co-workers employed one of the simplest configurations of McKenna, i.e. primary premixed flat-flame without introducing a secondary jet flame at the center of burner. Using $\mathrm{C}_{3} \mathrm{H}_{8} / \mathrm{O}_{2} / \mathrm{SiCl}_{4}$ mixtures, as shown in Fig. 2(b), they studied the synthesis of $\mathrm{SiO}_{2}$ nanopowders [271][272]. The experimental data of particle growth was consistent with that predicted by a simple Brownian collision-coalescence model for residence times ranging from 30 to 100 milliseconds, by using a sticking coefficient as 0.004 . Two decades later, Ehrman, Friedlander, and Zachariah (1998) [270] employed a similar primary premixed flame McKenna burner, as shown in Fig. 2(b), to generate $\mathrm{SiO}_{2} / \mathrm{TiO}_{2}$ nanocomposite nanoparticles $(\sim 15 \mathrm{~nm})$. The McKenna burner generally consists of a gravity sintered bronze plug that is encased in a stainless steel sleeve and sealed with O-rings. A radially uniform premixed flame was stabilized approximately $1.5 \mathrm{~mm}$ above the surface of the burner. To prevent sintering of the burner surface, a cooling coil circulating chilled house water was built into the plug during the gravity sintering step, positioned parallel to and one centimeter below the surface [270]. In this premixed flame environment, the high concentrations of radical species imply that the precursor oxidation chemistry will be dominated by reactions involving radicals, and therefore will be very fast 
relative to particle formation processes. Thus, for this flame configuration, chemical processes are not rate determining for particle size and do not affect the arrangement of species for mixed-oxides system.

In the early studies of Ulrich and co-workers, the McKenna premixed flat-flame burner was often used as a surrounding shroud to support a central secondary turbulent-jet diffusion flame [273]. Therefore, the McKenna burner can be easily modified to be a flame-assisted spray synthesis process to produce mixed oxide materials by introducing additional liquid or solid precursor reactants into the central tube. Other notable applications of the McKenna burner include the synthesis of non-oxide $\mathrm{Si}_{3} \mathrm{~N}_{4}$ nanoparticles by using $\mathrm{SiH}_{4} / \mathrm{NH}_{3} / \mathrm{N}_{2} \mathrm{H}_{4}$ reactants [245], along with the evaluation of electric effects on the synthesis of $\mathrm{SiO}_{2}$ nanoparticles [274].

Generally, the simple one-dimensional geometry of the McKenna flat-flame burner enables it to be one of the "prototypical" reactors for fundamental study of the evolution of particle growth, extending to the related model developments and validations [275]. The yield of $\mathrm{TiO}_{2}$ nanoparticles broadly ranges from 0.27 to $15.0 \mathrm{~g} / \mathrm{MJ}$-fuel, with product flux of $8-800 \mathrm{~g} / \mathrm{min} \cdot \mathrm{m}^{2}$, which can well simulate the nanoparticle conditions of industrial units in the laboratory (as seen in Table 1). For example, the simultaneous monitoring of the growth of both nanoparticles and aggregates was performed using a McKenna premixed flat flame, as characterized by in situ ultra-small-angle x-ray scattering [276], by rapid thermophoretic sampling with off-line analysis of transmission electron microscope images [277][278], and by in situ particle mass spectrometry (PMS) from molecular beam sampling to extract particles from low-pressure premixed flat flames [279][280]. However, so far the scalable application of McKenna burner has not been reported. 


\subsection{Hencken flat-flame Burner}

In parallel to the McKenna premixed flat-flame burner, the so-called Hencken flat-flame burner operating in the diffusion flame regime has been also widely used for calibration of both optical (e.g. laser diagnostics) and physical (e.g. thermocouples) probes in the combustion science community [265][281]. The surface-mixing Hencken burner, which consists of a ceramic honeycomb with hundreds of stainless steel tubes (with extra-small I.D. $\sim 0.5-1.5 \mathrm{~mm}$ ), can generate hundreds of multi-element, non-premixed- flamelets, producing an overall scalar profile that is radially-flat, uniform, steady, and nearly adiabatic under the correct flow conditions, as shown as primary flames in Fig. 2(c). Therefore, it is also termed as the Multi-Element Diffusion-flame Burner (MEDB) or Near-Adiabatic Flat-flame Burner in the combustion community [281][282]. Compared with the McKenna burner, the Hencken burner possesses several advantages. Firstly, it does not need an additional cooling coil system to prevent the sintering of the burner surface. Secondly, since it operates in the diffusion flame mode, global "lean" and "rich" flammability limits of the Hencken burner are absolutely broader than those of the McKenna burner. As such, more flexibility exists for adjusting operating conditions of hot gaseous environments for any existent applications, including diagnostics calibration, coal or particle fuel combustion, and flame synthesis. Thirdly, as mixing occurs outside the body of the burner, atmospheric flames using relatively high concentrations of pyrophoric precursors can be safely studied using the Hencken burner without risk of flashback. Fourthly, though the Hencken burner consists of a large number of small diffusion flames, the rapid mixing and subsequent formation of a slightly dimpled flame surface or sheet may be approximately described using adiabatic premixed equilibrium. 
Initially, the Hencken flat-flame burner was widely used to provide a uniform hot ambience, either $\mathrm{O}_{2}$ rich or $\mathrm{O}_{2}$ free, for studying the characteristics of coal devolatilization/combustion process, in which coal particles are entrained by a $\mathrm{N}_{2}$ carrier gas stream and then fed into a central tube with a relatively larger diameter (2 4 $\mathrm{mm}$ in contrast to $0.5 \sim 1.5 \mathrm{~mm}$ of Hencken elementary tube) [283]-[286]. The center coal-loaded jet stream forms a secondary diffusion flame that is much longer than the primary flat flame arrays, as shown in Fig. 2(c).

Considering the aforementioned advantages of the Hencken burner, Wooldridge and co-workers employed it for flame synthesis of nanomaterials, e.g., $\mathrm{SiO}_{2}, \mathrm{SnO}_{2}$, and $\mathrm{Au}$-doped $\mathrm{SnO}_{2}$ nanocomposites, etc. [282],[287]-[290]. Firstly, in their early design for producing single-component $\mathrm{SiO}_{2}$, the primary flat flame is solely employed, in which 173 stainless-steel fuel tubes (I.D. 0.508mm) carrying $\mathrm{H}_{2} / \mathrm{Ar} / \mathrm{SiH}_{4}$ mixtures, surrounded by 480 total oxidizer channels (each fuel tube by six oxidizer channel), was arranged in a square $2.54 \mathrm{~cm}$ Hastalloy honeycomb matrix. The multi-element flat flame is shrouded by $\mathrm{N}_{2}$ flow and a square optical chimney to isolate the synthesis environment and extend the high-temperature conditions. The typical operating conditions of the Hencken burner was reported to have temperatures in the core region of $\mathrm{T}=900$ to $1660 \mathrm{~K}$, global equivalence ratios of $\Phi=0.5$ to 2.2 , and silane loadings of 0.15 to $0.41 \%$ (mole basis, percentage of all $\mathrm{SiH}_{4} / \mathrm{H}_{2} / \mathrm{O}_{2} / \mathrm{Ar}$ reactants), resulting in the silica production rates of $1.2 \sim 4.1 \mathrm{mg} / \mathrm{s}$ (corresponding to $\mathrm{SiO}_{2}$ yields of $3.8 \sim 5.3 \mathrm{~g} / \mathrm{MJ}$-fuel or mass flux of $112 \sim 376 \mathrm{~g} / \mathrm{min} \cdot \mathrm{m}^{2}$ ) [282]. More recently, the second successful application of Hencken flat flame was to employ hundreds of fuel tube array (I.D. $=0.5 \mathrm{~mm}$ ) as a hot oxidative environment to support a gaseous synthesis process of $\mathrm{Au}$ - or $\mathrm{Al}$-doped $\mathrm{SnO}_{2}$ nanocomposite, in which a central fuel tube (I.D. $=0.85 \mathrm{~mm}$ ), isolated from the main fuel tube array, was used to feed both gas precursors 
(tetramethyl tin, TMT) and solid precursors (e.g., gold acetate) [288]-[290]. The results indicate that metal acetates can provide an excellent source for metal additives in flame synthesis environments, yielding gold particles encapsulated in a layer of $\mathrm{SnO}_{2}$ with excellent sensor response and time resolution.

As for further extended applications of Hencken burner, more recently, Zheng and co-workers [158][159] have demonstrated a new economical and scalable flame-assisted chemical vapor deposition (CVD) method (instead of flame aerosol synthesis) to grow one-dimensional (1D) metal oxide nanostructures by directly oxidizing metals in the postflame region of a multi-element flat flame. The 1-D metal oxide materials include aligned $\gamma-\mathrm{Fe}_{2} \mathrm{O}_{3}$ nanoflake, $\mathrm{CuO}$ nanoneedle arrays, and single, branched, and flower-like $\alpha-\mathrm{MoO}_{3}$ nanobelt arrays. The $\gamma-\mathrm{Fe}_{2} \mathrm{O}_{3}$ nanoflakes and $\mathrm{CuO}$ nanoneedles grow by a solid diffusion mechanism, are single or bicrystals, and are aligned perpendicularly to the substrate with a large surface coverage density. The growth rate, morphology, and surface coverage density of the 1D metal oxide materials can be flexibly controlled by varying the flame equivalence ratio $(0.8-1.4)$, the source temperature $\left(500-800^{\circ} \mathrm{C}\right)$, the growth substrate temperature $\left(400-700^{\circ} \mathrm{C}\right)$, and the material and morphology of the growth substrate. In addition, a novel flame synthesis method developed by Tse and co-workers [291]-[294] by utilizing a multi-element inverse diffusion burner, in which most of the fuel does not pass through the oxidation zone in underventilated flames, is demonstrated to be well suited for synthesizing carbon-based nanostructures .

These advantages and wide applications of the Hencken burner demonstrate its important role in future studies of flame synthesis. As mentioned above (Fig. 1), the competing mechanisms of collision-coalescence is of significance in determining the growing sizes and morphologies of 
synthesized nanoparticles. More than the characteristic collision time $\left(\tau_{\text {coll }}\right)$, the characteristic coalescence time ( $\tau_{\text {sint }}$, also known as sintering time) is very sensitive to the temperature. Claims from previous data have been made that the lateral thermal uniformity of the Hencken burner possesses comparable or better quality than that found in the McKenna burner [279][282], affording fundamental study of the coalescence/sintering rate of nanoparticles at high temperatures. The one-dimensional post-flame temperature dependence as a function of axial coordinate, greatly simplifies theoretical interpretation of experimental data. Among all benchmark burners, the Hencken burner, due to these distinct advantages, seems to provide an "ideal" platform for nano-aerosol characterization using in situ optical diagnostics, as well as doped synthesis of nanocomposites via. vapor-fed, liquid-fed or even solid-fed routes [294]-[296]. For example, Li and co-workers developed a new flame configuration, consisting of a secondary premixed jet flame (Bunsen burner) shrouded by a multi-element diffusion flame (Hencken burner), to demonstrate the in situ two-dimensional imaging of nucleation-coagulation processes of $\mathrm{TiO}_{2}$ in flames, for the first time, by using laser-driven localized nanoplasmas [297][298].

\subsection{Counterflow diffusion flame burner}

The counterflow diffusion flame burner, known as one of the typical stagnation-point flames, possesses a quasi one-dimensional geometry that is different than those of the McKenna burner and the Hencken burner. It was first proposed by Chung and Katz [299], then developed by Zachariah, Katz and co-workers [300]-[306], and later adapted by Rosner and co-workers [21][307][308], for the atmospheric pressure synthesis of both single oxides such as $\mathrm{SiO}_{2}, \mathrm{Al}_{2} \mathrm{O}_{3}$, and $\mathrm{TiO}_{2}$, and multi-component oxides, such as $\mathrm{TiO}_{2} / \mathrm{SiO}_{2}, \mathrm{Al}_{2} \mathrm{O}_{3} / \mathrm{SiO}_{2}$, and $\mathrm{SiO}_{2} / \mathrm{GeO}_{2}$. 
The burner consists of two vertically opposed nozzles of rectangular (or cylindrical) cross section separated by a certain distance (e.g. around $15 \mathrm{~mm}$ in [299][308]). The fuel (either $\mathrm{H}_{2}$ or $\mathrm{CH}_{4}$ diluted with inert gas) flows upward from the lower tube, and the oxidizer $\left(\mathrm{O}_{2}\right.$ diluted with inert gas) flows downward from the upper tube, forming a thin flame sheet with temperature that can be well controlled by adjusting the flow-rate of dilution gas, as shown in Fig. 2(d). The counterflow diffusion flame has several advantages as one of the bench-scale flame aerosol reactors. First, the counterflow burner provides a near-adiabatic flat flame (similar to the Hencken burner), reducing the complexity in modeling, where the lateral/radial temperature profiles in the burner core region have variations of less than $25 \mathrm{~K}$ ( $\sim 2 \%$ variations from mean value) [299]. Second, in contrast to uniformness of lateral/radial temperature profiles, the gradients of axial temperature on either side of the reaction zone is very high (ca. $4500 \mathrm{~K} / \mathrm{cm}$ ), which is larger than those characteristic of the post-flame cooling regions of the coflow diffusion flame burner, the McKenna flat-flame burner, and the Hencken flat-flame burner (commonly less than $100 \mathrm{~K} / \mathrm{cm}$ ) [21]. Therefore, further agglomeration of as-synthesized nanoparticles is limited, and the formation of spherical-shaped nanoparticles with low degree of agglomeration is enabled, though the sizes of primary particles are quite similar to those produced by other typical burners (as shown in Table 1). Hence, the counterflow burner serves as an "ideal" reactor for fundamental study of the formation mechanisms of nonagglomerated spherical particles.

In counterflow diffusion flames, the synthesized (or introduced) oxide particles are subjected to two main competing forces: viscous drag forces due to the gas flow, and thermophoretic forces due to the sharp temperature gradient. Thus, a particle stagnation plane (PSP) can be defined at a location where particles then have zero mean axial velocity, that is, the axial component of the particle 
thermophoretic velocity is equal to the axial component of the local gas velocity but of opposite direction. For example, in a 15mm-separation-distance counterflow burner under given conditions in Ref. [21], the PSP occurs at a distance of $z=8.7 \mathrm{~mm}$ above the fuel tube, as measured from the falloff of the light scattering signal, where the flame sheet (defined as the location of peak temperature) occurs at $\mathrm{z}=6.3 \mathrm{~mm}$, which is below PSP location. The gas stagnation plane (GSP) is estimated to be a little below the particle stagnation plane (i.e. $z=8.5 \mathrm{~mm}$ ) [21]. Another work reported their PSP occurring at $\mathrm{z}=10.2$ $\mathrm{mm}$ and the flame location at $\mathrm{z}=6.7 \mathrm{~mm}$, with the distance between the two nozzles also at $15 \mathrm{~mm}$ [308]. Within this flame structure, particles formed on the fuel side can pass through the high-temperature reaction zone and stop at the PSP plane. Consequently, by adjusting the positions of the PSP and the flame (as well as its temperature), the temperature-time history of the particles can be controlled to produce nanopowders with specific phases and sizes. For example, Xing et al. found that the mean "primary" particle diameters of $\mathrm{Al}_{2} \mathrm{O}_{3}$ ranges from 13 to $47 \mathrm{~nm}$ and the yields varies from $0.05-0.4$ g/MJ-fuel by using $\mathrm{Al}\left(\mathrm{CH}_{3}\right)_{3} / \mathrm{CH}_{4} / \mathrm{O}_{2} / \mathrm{N}_{2}$ counterflow diffusion flames [21]. Zachariah et al. found $d_{p}$ of $\mathrm{SiO}_{2}$ in the range of $30-150 \mathrm{~nm}$ in $\mathrm{SiH}_{4} / \mathrm{CH}_{2} / \mathrm{O}_{2} / \mathrm{Ar}$ counterflow diffusion flames [300].

In exploring the applications of the bench-scale counterflow burner, it is noted that the differences in the chemical reaction behavior of the precursors could affect particle size for single component oxide nanoparticles and arrangement of species for multicomponent nanocomposites. The precursor chemistry effect was successfully clarified by using a counterflow diffusion flame to comparatively study the formation of $\mathrm{SiO}_{2}$ particles from different precursors, i.e., silane, hexamethyl disiloxane (HMDS) and tetramethyl silane (TMS) [302]. By monitoring using in situ light scattering diagnostics, $\mathrm{SiO}_{2}$ particles formed from silane were detected at the earliest location in the flame, followed by silica particles formed 
from HMDS, and then by those from TMS, explaining the detailed reaction chemistry given in Section 6.1.

The precursor chemistry effect was further investigated in binary aerosol systems by synthesizing $\mathrm{SiO}_{2} / \mathrm{TiO}_{2}, \mathrm{SiO}_{2} / \mathrm{GeO}_{2}$, and $\mathrm{Al}_{2} \mathrm{O}_{3} / \mathrm{SiO}_{2}$ mixed oxides using the counterflow diffusion burner [304][305]. Light scattering observations showed that $\mathrm{TiO}_{2}$ particles form before $\mathrm{SiO}_{2}$ particles do during simultaneous seeding of $\mathrm{TiCl}_{4}$ and $\mathrm{SiCl}_{4}$.

Here we make a brief comparison between two kinds of flat flame configurations, i.e. counterflow diffusion and McKenna premixed flames. For the premixed flame, all gaseous species, including precursors, experience a short preheating zone prior to passing through the reaction zone with high concentrations of radical species such as $\mathrm{O}, \mathrm{OH}$, and $\mathrm{H}$. In contrast, for the counterflow diffusion flame with separated fuel/oxidizer flow, the temperature-time profile of the burner can be varied by adjusting the particle stagnation plane. The radical-release reactions intensively occur in the plane-like flame zone in the middle and diffuse to the surrounding. Thus, these radical-driven decomposition reactions are prone to happen in the narrow flame zone. In the case of multi-element flame synthesis with different precursor chemistry, the employment of a certain burner would make a difference in the particle morphology. These aspects are reviewed in detail later in Section 3.

\subsection{Premixed stagnation flame burner}

In contrast to the opposed-jet stagnation flow in counterflow burners, a jet-wall configuration provides another alternative to study pertinent chemical phenomena in planar (quasi one-dimensional), aerodynamically-strained flames. These stagnation flat flames provide an invaluable test apparatus for 
development, validation, and optimization of transport and kinetics models, as they can be simulated using a simple one-dimensional hydrodynamic model [309]. Since the mixture of fuel, oxidizer, precursor species, and inert gas flow together out from the nozzle toward the plate, the jet-wall stagnation flame mostly operates in premixed mode, and is thus termed premixed stagnation flame in the combustion community [310]. Figure 3 shows the axial profiles of temperature, velocity, and molar fractions of species $\mathrm{C}_{2} \mathrm{H}_{4}$ (Fuel) and $\mathrm{CO}_{2}$ (Product) along the centerline of a typical premixed stagnation flame (Mixture: $3.96 \%-\mathrm{C}_{2} \mathrm{H}_{4} / 26.53 \%-\mathrm{O}_{2} / 69.51 \%-\mathrm{Ar}$; Equivalence ratio: $\Phi=0.45$; Configuration: I.D. 1 cm nozzle with nozzle-substrate distance $3.4 \mathrm{~cm}$ ), as re-plotted from literature [311]. In the particle nucleation/growth zone after the reaction zone (close to peak temperature position), the axial temperature gradient is as large as $5 \times 10^{4} \mathrm{~K} / \mathrm{cm}$, which is larger than the $4500 \mathrm{~K} / \mathrm{cm}$ in the counterflow diffusion flame mentioned above, and dramatically larger than those in coflow diffusion flames produced by McKenna and Hencken burners ( $\sim 100 \mathrm{~K} / \mathrm{cm}$ without special quenching), as compared in Table 1. This steep temperature gradient implies a fairly large thermophoretic velocity that drives nanoparticle deposition on the substrate.

In the field of materials synthesis, premixed stagnation-point flames are of significance in many industrial applications, such as chemical vapor deposition (CVD) of polycrystalline diamond films, modification of thin polymer films, and alteration of metal surfaces to create carbides. For example, in the late 1990's, uniform diamond deposition was demonstrated by Murayama and Uchida in a fuel-rich acetylene-oxygen-hydrogen $\left(\mathrm{C}_{2} \mathrm{H}_{2} / \mathrm{O}_{2} / \mathrm{H}_{2}\right.$ mixture $)$ flat flame stabilized in the stagnation region of a molybdenum substrate [312][313]. Numerical simulation of the temperature profiles in the region near the deposition substrate indicated that this particular flame configuration can achieve uniform and 
high-quality polycrystalline films, along with the potential of scaling for the deposition of large-area films [314]. The simulation is validated by in situ temperature measurements using Coherent anti-Stokes Raman scattering (CARS) spectroscopy of $\mathrm{H}_{2}[315]$.

More recently, premixed stagnation flames have been developed for the one-step synthesis of ultrafine, high-purity nanoparticles (with sizes of $<10 \mathrm{~nm}$ ), with further fabrication of the related functional thin films by several research investigators [96][112],[316]-[324]. From these studies, it can be summarized that, in the jet-wall stagnation-point geometry, the cooled stagnation substrate provides many advantages for nanomaterial synthesis, such as: $(i)$ steep temperature gradient for ultrafine nanoparticle production, (ii) controllable particle temperature histories based on burner-substrate distance to reduce agglomeration, (iii) flame stabilization by stagnation wall, (iv) collection source for nanoparticles deposition by thermophoresis, $(v)$ adjustable substrate temperature for nanoporous film formation, and ( $v i)$ uniform electric field application. These advantages provide a sufficient reason that the jet-wall configuration, instead of the opposed-jet counterflow configuration, is commonly used in the premixed stagnation flame synthesis. (To the best of our knowledge, the application of counterflow premixed flames in materials synthesis is relatively scarce). However, the jet-wall configuration intrinsically results in nonadiabatic flames because of heat loss to the substrate wall, which is different than that the near-adiabatic counterflow premixed flame given the symmetry of the dual flame configuration. The cool stagnation substrate wall introduces a heat sink to the flow, and for sufficiently large degrees of cooling, along with sufficiently large rates of strain, extinction will be induced (Libby and Williams, 1983 [325]).

To stabilize the jet-wall premixed stagnation flame for a sufficiently long operational duration for 
materials synthesis, several techniques have been developed. Firstly, a natural stabilization method is to combine a McKenna premixed flat flame burner with a water-cooled substrate, forming a burner-stabilized premixed stagnation flame technique, as seen in Fig. 2(f.1). For example, Glumac and co-workers [258] employed this burner geometry at low pressures to synthesize loosely agglomerated nanopowders. Tse and co-workers added electric field application to the set-up, synthesizing crystalline anatase, ultrafine $\mathrm{TiO}_{2}$ particles (with 5-6 nm diameters of primary particles) by introducing titanium tetra-iso-propoxide (TTIP) vapor into $\mathrm{H}_{2} / \mathrm{O}_{2} / \mathrm{N}_{2}$ mixture [319][320]. One disadvantage of this technique lies in that the decomposed metal precursor may thermophoretically deposit in the small plug holes of burner head because the flame is stabilized at a close position that is only $\sim 1.5 \mathrm{~mm}$ distance from the burner surface. The cleaning of nanoparticles after each experimental run brings lots of inconvenience. Benefiting from the applications of stagnation flame diamond-film synthesis, Wang and co-workers adopted a two-concentric-tube coflow design, with inner tube of premixed fuel/oxidizer/precursor mixture and outer tube of inert gas, to stabilize the annular shear-layer at the edge of the flat flame [316]-[318] impinging on a substrate, as seen in Fig. 2(f.3). With a high flow velocity, they achieved a divergence-stabilized premixed stagnation flame. The novel use of a rotating disc substrate shortens the growth span of the particles, resulting in nanomaterials of greater tunability and monodispersity [311]. Moreover, a few of the sub-tubes can be arranged in the burner nozzle to prevent flame blow off and extinction, as shown in Fig. 2(f.2). Biswas and coworkers used a premixed methane-oxygen burner made of a $3 / 8$ O.D. stainless steel tube with three $1 / 8$ cylinders packed into the middle to achieve an optimal outlet velocity $(450 \mathrm{~cm} / \mathrm{s})$ through a $0.16 \mathrm{~cm}^{2}$ area [321][322]. Aiming at the scale-up of premixed stagnation flames, $\mathrm{Li}$ and co-workers employed a vane swirler (with swirl number $<0.6$ ), 
which is popular in the combustion community, to develop a novel, turbulent, premixed stagnation swirl flame (SSF) for the synthesis of high purity, ultrafine $\mathrm{TiO}_{2}$ nanoparticles [96][326]. As shown in Fig. 2(f.4), the improved flame stabilization and increased radial turbulent mixing from the swirl make the SSF a promising system for consistent and homogeneous large-scale production. It can produce ultrafine non-agglomerated nanoparticles of narrow size distribution, along with uniform deposition coverage. As seen in Table 1, if the sizes of primary particles are controlled less than $10 \mathrm{~nm}$, the yields of $\mathrm{TiO}_{2}$ nanoparticles is about $0.15-0.60 \mathrm{~g} / \mathrm{MJ}$-fuel (i.e. $50-200 \mathrm{~g} / \mathrm{min} \cdot \mathrm{m}^{2}$ with respect to burner nozzle area), exhibiting a scaling relationship of $Y d_{p}^{3}$ that is controlled by a complete coalescence (i.e. $\tau_{\text {coll }}>\tau_{\text {sint }}$ ). It means that mono-disperse ultrafine nanospheres are produced, since particles coalesce before another collision event occurs [10]. If the particle size further increases to $17 \mathrm{~nm}$, even a much larger $\mathrm{TiO}_{2}$ yield of $6.3 \mathrm{~g} / \mathrm{MJ}$-fuel can be achieved [318].

The renewed attention on the premixed stagnation flame in the combustion community can be attributed to not only meeting the need of controlling sizes or tailoring nanostructures with atomic precision, but also directly fabricating flame-made materials into functional devices, as detailed in Section 4. More recently, Li and co-workers proposed a new concept of one-step doping synthesis of mixed oxides nanomaterials by embedding the flame-assisted spray pyrolysis process into the premixed stagnation flame configuration [112]. Self-assembly, arising from the difference between the chemical time scales of palladium acetate decomposition and TTIP hydrolysis in the gas phase, generates ultrafine palladium particles $(<2.5 \mathrm{~nm})$ well dispersed on high surface-area $\mathrm{TiO}_{2}$ particles $(7-8 \mathrm{~nm})$. Indeed, even for the commonly liquid-fed flame spray pyrolysis process, the application of stagnation plate for nano-film fabrication has gained attention, which will be introduced in the following Section 2.6. 


\subsection{Flame spray pyrolysis and precursor feeding spray techniques}

As summarized above, flame spray pyrolysis (FSP) has been successfully employed to synthesize nanomaterials comprising nearly all metal elements of the periodic table, including oxides, salts, and even pure metals. The laboratory-scale reactors have production rates of a few grams per hour. The rapid development of FSP not only meets the needs of exploring low volatility and economical precursors, but also plays an important role in making functional nanocomposites by flexible doping in precursor solutions. The criteria for precursor selection are also summarized in the introduction section of this paper. In view of several review papers on FSP technology [5][26][28] available, here, we only briefly focus on burner techniques and scale-up studies of FSP.

In a typical FSP reactor, as displayed in Fig. 2(e) and in more detail in Fig. 4(A), metallic precursors dissolved in a liquid fuel are delivered through the center capillary of a two-fluid nozzle (970/4-S32; Schlick-Düsen); meanwhile, pure oxygen (or sometimes air) is utilized as dispersion gas and atomizes the liquid precursor into fine droplets with mass median diameters of several micrometers [41],[199],[327]-[329]. The sprayed droplets of a precursor/fuel mixture are ignited by six surrounding oxy-methane flamelets, which serve as pilot flames for holding stable combustion. The FSP burner is centered in the cylindrical reactor chamber and surrounded by a homogenized co-flow of ambient air that satisfies the entrainment requirement of the spray flame and cools the aerosol before powder collection with a bag-house filter. The fundamental studies on combustion characteristics of isolated micron-sized precursor/solvent droplets in FSP burner are experimentally investigated. In contrast to

pure solvent dropts that burn uniformly and quasi-steady, these precursor/solvent droplets in FSP 
possess a kind of disruptive combustion behavior [330]. The mechanism of disruptive droplet burning is similar to that of slurry droplets, consisting of three main steps: (1) diffusion-controlled burning of the high-volatile solvent, (2) viscous-shell formation due to decomposition of the low-volatile metal precursor, and (3) subsequent disruption due to heterogeneous nucleation. It was found that the concentration and decomposition characteristics of the metal precursor are of signicance in determing the time sequence of these three steps.

Scale-up studies of the FSP reactor have been successively done by Mueller et al. [199][327], and Gröhn et al. [328][329]. Primarily, Mädler et al. conducted a parameter study and examined the roles of process parameters on the characteristics of product particles, achieving $\mathrm{SiO}_{2}$ production rate of $9 \mathrm{~g} / \mathrm{h}$ at a liquid HMDSO/ethanol feed rate of $1 \mathrm{ml} / \mathrm{min}$ [41]. Pure oxygen as oxidant makes the droplets burn much faster than that with air; thus, the product particles experience longer residence times at higher temperatures, promoting the formation of homogeneous and highly crystalline particles by sintering. The specific surface area can be well controlled by oxidant or fuel composition, flow rate, and precursor concentration. For scale-up, a larger spray angle and a broader flame was found to allow higher production rates of nanoparticles with similar particle sizes than that found in lab-scale reactors. Therefore, the particular design of the swirler to enlarge the spray angle and broaden the spray flame are considered for the atomizers, as shown in Fig. 4(A). For example, in this design, as the feeding rate of precursor/fuel mixture $\left[\mathrm{Zr}\left(\mathrm{C}_{3} \mathrm{H}_{7} \mathrm{O}\right)_{4}, 70 \mathrm{wt} \%\right.$ in n-propanol that is diluted in ethanol at a concentration of $0.5 \mathrm{M}$ ] increases from 27.1 and $81.1 \mathrm{~mL} / \mathrm{min}$, under a fixed $\mathrm{O}_{2}$ dispersion gas flow rate of $50 \mathrm{~L} / \mathrm{min}$, the $\mathrm{ZrO}_{2}$ powder production rate increases from 100 to $300 \mathrm{~g} / \mathrm{h}$, meanwhile the product primary particle diameter increases from 12 to $26 \mathrm{~nm}$ [199]. Gröhn et al. [329] further demonstrated that the product 
primary particle, agglomerate size, and crystallinity can be conserved during the scale-up of FSP from $\sim 100$ to $500 \mathrm{~g} / \mathrm{h}$ of zirconia, provided that the precursor and the dispersion flow rate are increased by the same factor (i.e. the gas-to-liquid mass ratio are held constant). In addition, the yields of nanoparticles by FSP, about 4.0-24 g/MJ-fuel, are also very close to those in other VAFS process, as seen in Table 1 .

More recently, to meet the need for scalable and economical manufacturing of nanomaterials by FSP, a novel atomization concept was presented [331]. It is a combination of a liquid pressure swirl nozzle (namely liquid hollow cone nozzle) and a swirl dispersion gas nozzle (namely discrete jet nozzle). As seen in Fig. 4(B), a pressure swirl nozzle is applied to create a liquid hollow cone that is suitable for liquid precursor/fuel mixtures; and in the second step, a different swirl dispersion gas nozzle is utilized to enhance the atomization of the liquid phase and to influence the spray cone formation and geometry. In atomization, $\alpha$ and $\beta$ represent the gas inclination angle and gas swirl angle, respectively, for the swirl dispersion gas nozzle; meanwhile $\gamma$ is the liquid hollow cone angle of the pressure swirl nozzle. By using computational fluid dynamics (CFD) simulation, it was found that the gas swirl angle has a significant influence on the axial gas velocity distribution, the direction of the gas flow, and the stabilization of spraying. Based on the experimental data in terms of mass median droplet diameter, the dispersion gas nozzle (number of holes $/ \alpha / \beta)$ of $8 / 30^{\circ} / 15^{\circ}$, together with the pressure swirl nozzle $\left(\gamma=70^{\circ}\right)$ can result in small droplets of $17.6 \mu \mathrm{m}$ that is much closer to the droplet size of conventional lab-scale flame spray pyrolysis-nozzles.

Electro-hydrodynamic (EHD) atomization, i.e. electrospray atomizer, which is capable of generating monodisperse aerosols with much smaller droplets than any other spray methods, may possibly meet the need of controlling sizes or dispersity with sufficient accuracy [195][332]-[334]. 
Electrospray atomizer relies upon a strong electric field to force the liquid column inside a capillary to form a cone. The electric charges on the droplets result in repulsive forces that delay cloud coalescence, which, combined with the Rayleigh jet break-up into quasi-monodisperse droplets, enables the atomizer to be attractive in nanomaterial synthesis [195][335][336]. More recently, Geier and Parker [195] employed an electrospray atomizer in flame synthesis of yttria-stabilized zirconia (YSZ) nanoparticles. Fig. 4 (C) shows a schematic of such a spray configuration with nozzles pointed radially to the counter electrode in the centerline and with a concentric ring extractor to minimize cross-talk between neighboring sources, providing some shielding between the individual jets before their break-up. This electrospray system further uses a Hencken flat-flame burner, as described in detail in Section 2.3, to create a vertically-directed hot gas flow for combustion of the generated droplets [195]. Despite the controllably narrow particle size distribution of nanoparticles by electrospray flame synthesis, the small production rates limit the applicability of the process for mass quantities.

In addition to the two-fluid (nozzle) and electrospray atomizers, ultrasonic atomizers are also commonly adopted in practice. The setup for ultrasonic atomization is simple and highly-efficient, and is also known as ultrasonic nebulizer. With the driving force coming from ultrasonic vibration, a narrow nozzle is no longer necessary in the design to help break up the liquid and form the droplets. Ultrasonic atomization is capable of atomizing an even wider range of solutions, including slurry and suspensions. The droplet size of the ultrasonic atomization can range from several to hundreds of micrometers, depending on the ultrasonic energy (frequency), carrier gas velocity, and liquid properties (like surface tension, density, and viscosity). Early research usually yielded sub-micron particles from flame synthesis via ultrasonic atomization; and these particles are believed to be directly precipitated from 
single droplets [337]. Later, with the combined consideration of droplet evaporation and precursor chemistry, nano-sized oxide particles were also obtained through ultrasonic atomization, involving rapid solvent evaporation at the first stage, followed by precursor decomposition in the gas phase $[48][116][186][338]$. Granted the versatility and flexibility of ultrasonic atomization, the production of a wide range of oxide particles with controlled morphology can be realized, including hollow particle, doughnut particle, encapsulated particle, and 'hairy' particle, as elaborated in the review paper by Nandiyanto and Okuyama [339].

\section{Progress on the synthesis of metal oxide nanocomposites}

Under a new need for producing functional nanocomposites, flame aerosol synthesis, with its strong flexibility, can not only precisely deposit a noble metal cluster onto a pristine oxide support like $\mathrm{TiO}_{2}$ and $\mathrm{Al}_{2} \mathrm{O}_{3}$ with atomic accuracy [68][112], but can also readily dope or fabricate the mixed oxides or even their supported noble metals to achieve a particular functional utilization [51][64],[146]-[150],[340]-[345]. Several previous reviews have specially concentrated on the

applications of mixed metal oxide catalysts [5][25][26][28]. Here, particularly from the point of view of the morphological structures of doped or fabricated nanocomposites, we briefly review the progress on supported metal nanocatalysts, photocatalysts, sensing nanomaterials, and nanophosphors, respectively. A doping diagram summarizing flame aerosol synthesis of nanocomposites with desired structures is presented thereafter at the end of this section. 


\subsection{Synthesis of supported metal nanocatalysts}

Among various kinds of flame-made catalysts, supported metal nanocatalysts have attracted wide notice and are extensively studied for special construction and excellent catalytic activities. Supported noble metals are widely used as catalysts in energy conversion processes, such as fine chemical synthesis, refining of fuels to low-temperature combustion of hydrocarbons, and treatment of exhaust gas. For these classes of catalysts, dispersion of the precious metal (e.g., Pt, Pd, and Rh) on the oxide support is especially critical because of the high expense of the metal. Therefore, a specially supported structure of noble metal and oxide support, as shown in Fig. 5 (A, B, C), is needed for the design of catalysts, where small active metal clusters are generally dispersed on high surface area oxide supports such as $\mathrm{TiO}_{2}, \mathrm{Al}_{2} \mathrm{O}_{3}$, and $\mathrm{SiO}_{2}$, respectively. The observed catalytic activities for such supported catalysts were significantly higher than those expected simply by summing the contributions from the oxide supports and the pure metals. This enhancement can be ascribed to the activity at the special oxide-metal interface, coupled with the activities of the oxide support and the noble metal. Flame-made catalysts have shown superior catalytic performances compared to those prepared by conventional impregnation methods. For example, for enantioselective hydrogenation by $\mathrm{Pd} / \mathrm{Al}_{2} \mathrm{O}_{3}$ catalysts, the rate and enantioselectivity of hydrogen pretreatment of the flame-made catalysts at $500^{\circ} \mathrm{C}$ was greatly improved, whereas the corresponding wet-phase-derived catalysts showed only a comparatively weak beneficial effect [346]. The superior catalytic performances may be attributed to two aspects: $(i)$ the well-dispersed structures of metal-support with ultra-high surface area (as high as $200 \mathrm{~m}^{2} / \mathrm{g}$ [112]) that are shown in Fig. 5, and (ii) the formation of special interfaces and strong bindings (known as 
metal-support interaction) due to the atomic-level fabrication of binary components under high temperature, often considered as the reason for unique catalytic behaviors [347].

Moreover, factors affecting the catalytic activities also include the acidity of supports, precious metals, promoter, stabilizer or additive, etc [73][83][347], which is beyond the scope of this review, particularly considering several existent reviews on this topic that readers may be interest in [26][28].

\subsection{1 $\mathrm{TiO}_{2}$ support}

The strong metal-support interaction has been directly linked to the presence of electronic defects that can be prepared with ease on the surfaces of reducible oxides such as $\mathrm{TiO}_{2}$, as well as on other kinds of transition metal oxides [348]. Basically, this strong metal-support interaction is essential to sustaining high catalytic activity under demanding catalyst operation conditions (i.e., high temperature, high water vapor pressure, etc.). Anchoring the active metal components to these electronic defects has been documented to have strong interaction between the active metals and the defects of the reducible oxides, fundamentally determining the dispersion, morphology, and, catalytic activity of metal clusters. For example, the correlation between the number of oxygen defects on the $\mathrm{TiO}_{2}$ support and the dispersion and morphology of nanosized metal particles has been clearly demonstrated by density functional theory (DFT) calculations [349].

Innovative FASP design of highly-quenched premixed stagnation-point swirl flame (SSF) system based on atomic-level assembly (via controlling time scales of reaction, nucleation, and coagulation of different metal oxides) was used to synthesize well-dispersed palladium clusters on the surface of $\mathrm{TiO}_{2}$ nanoparticles with diameters below $10 \mathrm{~nm}$ [112]. As shown in Fig. 5 (A), through this spray flame 
synthesis, the ultrafine palladium particles $(<2.5 \mathrm{~nm})$ are well dispersed on the high surface-area $\mathrm{TiO}_{2}$ nanoparticles $(7-8 \mathrm{~nm})$. These flame-made nanocatalysts have shown superior catalytic performances compared to those prepared by conventional impregnation methods. The apparent activation energy of the FSP-deposited $\mathrm{Au} / \mathrm{TiO}_{2}$ catalyst is similar to the values reported in the literature for oxidation of $\mathrm{CO}$ over supported gold catalysts; however, the surface area is more than twice of that usually obtained in spray flame using quench cooling and filter-collected particles [350]. In addition, for flame-made $\mathrm{Pt}-\mathrm{Co} / \mathrm{TiO}_{2}$ catalysts, the addition of $\mathrm{Co}$ led to a higher amount of Pt terrace atoms being formed on the catalyst surface, which promoted the selectivity towards ethylnitrobenzene. The positive effect of Co addition can be observed when the catalysts were reduced at $500^{\circ} \mathrm{C}$. Both hydrogenation activity and selectivity of vinylaniline over $\mathrm{Pt}-\mathrm{Co} / \mathrm{TiO}_{2}$ were drastically increased and surpassed those of monometallic $\mathrm{Pt} / \mathrm{TiO}_{2}$ because of the strong interaction between $\mathrm{Pt}-\mathrm{Co}$ and the migration of $\mathrm{TiO}_{\mathrm{x}}$ species [351].

\subsection{2 $\mathrm{Al}_{2} \mathrm{O}_{3}$ support}

The most frequently used support for noble metals is inert $\mathrm{Al}_{2} \mathrm{O}_{3}$, such as $\gamma-\mathrm{Al}_{2} \mathrm{O}_{3}$. Different from reducible oxides (e.g., $\mathrm{TiO}_{2}$ mentioned above and $\mathrm{CeO}_{2}$ to be discussed later), electronic defects are not present on $\gamma-\mathrm{Al}_{2} \mathrm{O}_{3}$. However, it seems that the coordinately unsaturated pentacoordinate $\mathrm{Al}^{3+}\left(\mathrm{Al}^{3+}{ }_{\text {penta }}\right)$ centers present on the (100) facets of $\gamma-\mathrm{Al}_{2} \mathrm{O}_{3}$ surfaces can effectively anchor noble metal clusters [352] Noble metals $(\mathrm{Ru}, \mathrm{Rh}, \mathrm{Pt}, \mathrm{Pd})$ on $\mathrm{Al}_{2} \mathrm{O}_{3}$ were made and tested in the catalytic partial oxidation of methane. The ignition of the reaction towards carbon monoxide and hydrogen depended on the noble metal constituents and their loading. The selectivity of these noble metal catalysts towards $\mathrm{CO}$ and $\mathrm{H}_{2}$ 
was similar under the conditions examined (methane:oxygen ratio 2:1, temperature from 300 to $500{ }^{\circ} \mathrm{C}$ ), exceeding significantly those of gold and silver containing catalysts [68]. $\mathrm{Rh} / \mathrm{Al}_{2} \mathrm{O}_{3}$ was found to show an enhanced and stable activity in the partial oxidation of methane [205]. The $\mathrm{Al}_{2} \mathrm{O}_{3}$ support inhibits $\mathrm{Rh}$ oxidation, maintaining $\mathrm{Rh}$ in its metallic state, which is preferable for high syngas formation. In addition, as mentioned above, flame-made $\mathrm{Pd} / \mathrm{Al}_{2} \mathrm{O}_{3}$ catalysts exhibited superior catalytic performances for enantioselective hydrogenation compared to corresponding wet-phase-derived catalysts [347]. Again, these enhanced catalytic performances can be attributed to the special interface and binding resulting from the atomic-level precision of assembly for binary metal-oxide systems by FSP or other kinds of spary flame synthesis techniques [347].

It should be noted that bimetallic or trimetallic supported catalysts have been also prepared by FSP as seen in Fig.6 (B), showing better performance than single metal supported ones. Flame-made $\mathrm{Pt}-\mathrm{Sn} / \mathrm{Al}_{2} \mathrm{O}_{3}$ catalysts with large pores exhibited better catalytic activity and stability than the ones made by impregnation in the dehydrogenation of propane, without altering the selectivity to propylene [354] . The presence of large pores in the flame-made catalysts not only facilitated diffusion of the reactants and products but also lessened the amount of carbon deposited during reactions. Bimetallic Pd-Pt nanoparticles supported on alumina were also prepared by the FSP method [69]. The noble metal components of the materials were predominantly in the oxidized state and well dispersed in the alumina matrix. Small bimetallic Pd-Pt alloy particles $(<5 \mathrm{~nm})$ supported on $\mathrm{Al}_{2} \mathrm{O}_{3}$ ceramic nanoparticles are then produced after reduction. The addition of small amounts of platinum made the palladium particles more resistant against the sintering at high temperatures and further lowered the deactivation during low-temperature methane combustion. The catalytic properties of the flame-made trimetallic 
nanocrystalline Pt-Sn-X/Al $\mathrm{O}_{3}(\mathrm{X}=\mathrm{Ce}, \mathrm{Zn}$, and $\mathrm{K})$ catalysts were investigated in the dehydration of propane [355]. It was found that the addition of Ce during FSP synthesis resulted in higher Pt dispersion as well as improved catalytic activity and stability than the non-promoted $\mathrm{Pt}-\mathrm{Sn} / \mathrm{Al}_{2} \mathrm{O}_{3}$. The mechanism for forming the trimetallic nanoparticles during one-step flame synthesis seemed to be strongly dependent on the difference between the vapor pressures of the metals and the alumina support.

In addition, mixed oxide supports are also of great interest. For example, FSP-made $\mathrm{SiO}_{2} / \mathrm{Al}_{2} \mathrm{O}_{3}$ mixed support can generate extra pseudo-bridging bonds, enhancing their Brøsted acidity to strengths that are similar or even higher than those encountered in conventional zeolites [347]. The molar ratio of strong and weak acid sites can be easily adjusted for desired reactions by varying the aluminum content. Then, palladium nanoparticles dispersed on silica-alumina $(\mathrm{Pd} / \mathrm{SA})$ with tunable surface acidity, with supported structure shown in Fig. 5(C), were prepared by one-step FSP [73]. Quantitative solid-state NMR spectroscopy of the catalysts with different $\mathrm{Si} / \mathrm{Al}$ ratio revealed that the concentration of Brøsted acid sites in these catalysts increases with increasing aluminum content up to 70 at $\%$. The support acidity directly influenced the electronic properties of the Pd nanoparticles as proved by DRIFT spectroscopy with $\mathrm{CO}$ as probe molecule. $\mathrm{Pd} / \mathrm{SA}$ with $15 \mathrm{at} \%$ aluminum (Pd/SA-15) exhibited excellent chemoselectivity for $\mathrm{C}=\mathrm{O}$ bond hydrogenation of acetophenone. Further enhancement of the support acidity as achieved in $\mathrm{Pd} / \mathrm{SA}-30$ and $\mathrm{Pd} / \mathrm{SA}-70$ further increased the reaction rate and promoted the competing hydrogenation of the aromatic ring [73]. The tunable acid properties of $\mathrm{SiO}_{2} / \mathrm{Al}_{2} \mathrm{O}_{3}$ may promote its application as a solid acid and as an acidic support in the chemical industry. 


\subsubsection{Perovskites $\left(\mathrm{ABO}_{3}\right)$ support}

These flame-made mixed oxide catalysts (or supports) also include perovskites and spinel. Perovskites are a kind of mixed oxides with general formula $\mathrm{ABO}_{3}$, where, in catalytic applications, $\mathrm{A}$ usually represents a lanthanide ion and $\mathrm{B}$ a transition metal ion. Both $\mathrm{A}$ and $\mathrm{B}$ can be partially substituted by other elements. Perovskites with high thermal stability are attractive for high temperature processes, such as catalytic combustion of natural gas in turbines for electricity generation.

La-based perovskites (such as $\mathrm{LaCoO}_{3}, \mathrm{LaMnO}_{3}$, and $\mathrm{LaFeO}_{3}$ ) have drawn particular interest because their catalytic properties can be easily tuned by substituting a small fraction of A-site atoms with other cations such as $\mathrm{Ba}, \mathrm{Ce}$, or $\mathrm{Sr}$ [356]. Perovskitic $\mathrm{LaCoO}_{3}$ mixed-metal oxides have been prepared in nanoparticle-size powder form, and the results showed that the higher the initial sintering of catalyst particles within the flame, the higher the thermal stability [146]. A set of perovskite-type catalysts of general formula $\mathrm{LaBO}_{3 \pm \delta}(\mathrm{B}=\mathrm{Co}, \mathrm{Mn}, \mathrm{Fe})$ have been prepared by flame-hydrolysis. These catalysts were characterized as nanometer-size particles $(20 \sim 60 \mathrm{~nm})$, with relatively high surface area (ca. $20 \mathrm{~m}^{2} / \mathrm{g}$ ), high thermal stability, and high phase purity, demonstrating high activity for catalytic flameless combustion of methane [357].

There are further modifications of the $\mathrm{LaBO}_{3 \pm \delta}(\mathrm{B}=\mathrm{Co}, \mathrm{Mn}, \mathrm{Fe})$ to improve the performances. Perovskite-type $\mathrm{La}_{1-x} \mathrm{Ce}_{x} \mathrm{MnO}_{3}(x=0-10 \%)$ catalysts were prepared by FSP. The introduction of $\mathrm{Ce}^{4+}$ resulted in modification of the chemical states of both B-site ions and oxygen species and also facilitated the reducibility of the perovskite [359]. The perovskite $\mathrm{La}_{1-x} \mathrm{Ce}_{x} \mathrm{MnO}_{3}$ with $x$ equal to 0.1 exhibited the highest activity, with a structure shown in Fig. 5 (D) [358]. Perovskite-type catalysts of general formula 
$\mathrm{ABO}_{3 \pm \delta}(\mathrm{A}=\mathrm{La}, \mathrm{Y} ; \mathrm{B}=\mathrm{Mn}, \mathrm{Fe})$ containing $2 \mathrm{wt} \% \mathrm{Pd}$ were produced by FSP. Among the investigated perovskite-type oxides, $\mathrm{YFeO}_{3 \pm \delta}$ exhibits the best performance for methane oxidation under stoichiometric conditions in the presence of $\mathrm{Pd}$, likely because of the high accessibility of Pd exposed at its surface [358]. $\mathrm{La}_{1-\mathrm{x}} \mathrm{M}_{\mathrm{x}} \mathrm{CoO}_{3 \pm \delta}$ with $\mathrm{M}=\mathrm{Ce}, \mathrm{Eu}$ and $\mathrm{x}=0,0.05,0.1,0.2$ were investigated. All samples were nanostructured, thermally very stable, and characterized by highly-crystalline perovskite-like structure and high surface area. The higher activity of Ce-doped catalysts as compared to those doped with Eu was found to be related to the strength of the bonds between oxygen and Co ions [360].

\subsubsection{Spinel $\left(\mathrm{AB}_{2} \mathrm{O}_{4}\right)$ support}

Spinel-type oxides are compounds with the general chemical formula $\mathrm{AB}_{2} \mathrm{O}_{4}$. Basically, the $\mathrm{A}$ ion is tetrahedrally coordinated, and the B ion octahedrally coordinated, with inverse spinel structures possible as well, where B ions, partially or fully, occupy tetrahedral positions and A ions reside in the octahedral sites. Many elements can be accommodated in spinel or spinel-like structures, allowing for a wide array of possible spinel formulations. Thus, there exists a correspondingly large flexibility for tailoring these oxides for specific applications, such as catalysts and sensors (as described in the later section). Flame-made $\mathrm{AB}_{2} \mathrm{O}_{4}$ has exhibited homogeneous single-crystal structure.

FSP of a Pd-based combustion catalyst was demonstrated, where homogenous spinel crystal $\mathrm{MgAl}_{2} \mathrm{O}_{4}$ acted as support [62]. During the heating and cooling cycles of methane conversion, both $\mathrm{Pd}-\mathrm{MgAl}_{2} \mathrm{O}_{4}$ and $\mathrm{Pd}-\mathrm{Al}_{2} \mathrm{O}_{3}$ retain much of their surface area, whereas $\mathrm{TiO}_{2}$ and $\mathrm{CeO}_{2}$ supports strongly sintered with large loss of surface area. 
Spinel-like oxides with the general formula $\mathrm{MgAl}_{2-x} \mathrm{M}_{x} \mathrm{O}_{4}(\mathrm{M}=\mathrm{Mn}, \mathrm{Fe}, \mathrm{Co})$ were synthesized in a one-step FSP. These oxides showed improved resistance to sintering upon increased alumina content. XPS data revealed the surface enrichment of the transition metal constituent, which favorably increased the fraction of transition metal exposed to the environment. By tuning the fraction of transition metal in the mixed oxides, nanocomposite materials were obtained with a good balance between thermal stability and activity in the catalytic combustion of methane. The activity of the materials increased in the order $\mathrm{Fe}<\mathrm{Co}<\mathrm{Mn}[130]$.

\subsection{5 $\mathrm{ZrO}_{2}, \mathrm{CeO}_{2}$, and $\mathrm{ZrO}_{2}-\mathrm{CeO}_{2}$ support}

In addition to $\mathrm{TiO}_{2}$ and $\mathrm{Al}_{2} \mathrm{O}_{3}$, which are the most widely used supports, there are also $\mathrm{ZrO}_{2}, \mathrm{CeO}_{2}$, and even their mixed oxide (i.e. $\mathrm{ZrO}_{2}-\mathrm{CeO}_{2}$ ). Flame-derived bimetallic $\mathrm{Pt}-\mathrm{Pd} / \mathrm{ZrO}_{2}$ catalysts were reported to exhibit excellent catalytic performances in solvent-free hydrogenation of alicyclic and aromatic ketones, such as cyclopentanone and acetophenone [75]. The Pt-Pd catalysts have a high dispersion of $\mathrm{Pt}$ and $\mathrm{Pd}$, and their metal loading can be easily controlled on the $\mathrm{ZrO}_{2}$ surface. More importantly, the catalysts can be reused without significant loss in activity and selectivity after appropriate treatment. The catalytic activities of the FSP-made $\mathrm{Co} / \mathrm{ZrO}_{2}$ catalysts for $\mathrm{CO}$ hydrogenation were much higher than those compared to $\mathrm{Co} / \mathrm{ZrO}_{2}$ prepared by the conventional impregnation method [361]. Moreover, the hydrogenation rates of the FSP-made $\mathrm{Co} / \mathrm{ZrO}_{2}$ catalysts increased with increasing Co loading and precursor feed rate. The improvement of catalytic activity and $\mathrm{C}_{2}-\mathrm{C}_{6}$ selectivities of the flame-made catalysts was attributed to the higher number of Co metal active sites and lower interaction between $\mathrm{Co} / \mathrm{CoO}$ and $\mathrm{ZrO}_{2}$ support. For flame-made $\mathrm{V} / \mathrm{ZrO}_{2}$ [101], low temperature $\mathrm{NO}$ catalytic 
reduction activity would indicate that an optimal dispersion of monomeric $\mathrm{VO}_{\mathrm{x}}$ species on zirconia is attained with $15 \% \mathrm{~V}$ in the $\mathrm{V} / \mathrm{ZrO}_{2}$ catalyst. Mixed oxides of $4 \% \mathrm{Cu} / \mathrm{Ce}_{x} \mathrm{Zr}_{1-x} \mathrm{O}_{2}(\mathrm{x}=0,0.25,0.50,0.75$, 1) were assessed for the equimolar reduction of NO by CO (3000 ppm each, space velocity $\left.50,000 \mathrm{~h}^{-1}\right)$ [162]. It was demonstrated that incorporation of $\mathrm{Zr}^{4+}$ in the form of solid solution with $\mathrm{CeO}_{2}$ stabilizes the $\mathrm{Cu}^{+}$species, which was in turn beneficial for the initial reductive chemisorption of $\mathrm{NO}$ to $\mathrm{N}_{2} \mathrm{O}$.

$\mathrm{NO}_{\mathrm{x}}$ storage-reduction (NSR) catalysis is the most promising concept for reducing $\mathrm{NO}_{\mathrm{x}}$ under lean conditions. $\mathrm{Pt} / \mathrm{Ba}$ catalysts for $\mathrm{NO}_{\mathrm{x}}$ storage-reduction (NSR) supported on ceria/zirconia were prepared by two-nozzle FSP [203]. The as-prepared material consisted of intimately mixed agglomerates of $\mathrm{BaCO}_{3}$ and $\mathrm{Ce}_{\mathrm{x}} \mathrm{Zr}_{1-\mathrm{x}} \mathrm{O}_{2}$ particles, as shown in Fig. 5(E). The low thermal stability of $\mathrm{BaCO}_{3}$ resulted in high NOx storage capacity, whereas the support composition $\left(\mathrm{Ce}_{\mathrm{x}} \mathrm{Zr}_{1-\mathrm{x}} \mathrm{O}_{2}\right)$ strongly affected the NOx reduction activity of $\mathrm{Pt}$. Generally, the flame-made $\mathrm{Pt} / \mathrm{Ba} /$ ceria-zirconia NSR catalysts are efficient for NOx storage; however, further investigations in the presence of real exhaust gases also containing $\mathrm{CO}_{2}$, $\mathrm{H}_{2} \mathrm{O}$, and $\mathrm{SO}_{2}$ are needed to fully assess the potential of flame-made $\mathrm{Pt} / \mathrm{Ba} /$ ceria-zirconia for $\mathrm{NOx}$ storage.

\subsubsection{Noble-metal free oxide catalysts with core-shell structures}

Nano- $\mathrm{Ce}_{x} \mathrm{Zr}_{1-x} \mathrm{O}_{2}$ and $\mathrm{Al}_{2} \mathrm{O}_{3}-\mathrm{Ce}_{x} \mathrm{Zr}_{1-x} \mathrm{O}_{2}$ materials made by FSP are not only excellent supports for noble metals, but also themselves offer surprisingly superior activities for both $\mathrm{NO}_{\mathrm{x}}$ reduction and hydrocarbon oxidation in high-throughput screening studies, as well as continuous flow catalytic studies [362]. The flame-made $\mathrm{CeO}_{\mathrm{x}} / \mathrm{Al}_{2} \mathrm{O}_{3}$ and $\mathrm{ZrO}_{2} / \mathrm{Al}_{2} \mathrm{O}_{3}$ nanocomposites usually exhibit a complex core-shell structure where the alumina coats crystals of ceria or zirconia or their solid solutions 
$\mathrm{Ce}_{x} \mathrm{Zr}_{1-x} \mathrm{O}_{2}$, as shown in Fig. 6 (A), with formula $\left(\mathrm{Ce}_{0.7} \mathrm{Zr}_{0.3} \mathrm{O}_{2}\right)_{0.5} /\left(\mathrm{Al}_{2} \mathrm{O}_{3}\right)_{0.5}$. These oxide catalysts offer activities comparable to that of traditional $\mathrm{Pt} / \mathrm{Al}_{2} \mathrm{O}_{3}$ catalysts but without the addition of Pt. Thus, they are Pt-free, yet still active for extremely important emission control reactions, especially considering that they are only first-generation raw materials. At higher $\mathrm{CeO}_{2}$ contents, core-shell particles are observed with $\mathrm{CeO}_{2}$ cores, but the shells contain considerable amounts of $\mathrm{Ce}^{3+}$ species, probably as $\mathrm{CeAl}_{11} \mathrm{O}_{18}$. It is possible therefore to envision that, at low $\mathrm{Al}_{2} \mathrm{O}_{3}$ contents, the resulting shells contain considerable quantities of $\mathrm{Ce}^{3+}$ and possibly $\mathrm{Zr}^{3+}$, which may contribute to the favorable activity.

How does one make a complex core-shell structure? For one, temperature has been experimentally demonstrated to be a good controlling parameter by Wang and co-workers [12]. $\mathrm{CeO}_{2}$ nanospheres doped with varied amounts of titanium were synthesized through FSP of a solution made by dissolving cerium carbonate into propionic acid with stoichiometric amounts of TTIP. The synthetic strategy of generating the nanoparticles by flame at about $2500^{\circ} \mathrm{C}$ facilitates the crystallization of the $\mathrm{CeO}_{2}(\mathrm{melting}$ point $2400^{\circ} \mathrm{C}$ ), while maintaining the $\mathrm{TiO}_{2}$ (melting point $1843^{\circ} \mathrm{C}$ ) in a molten state. Therefore, the (molten) titanium oxide shell encapsulates the inner ceria core and accommodates a spherical morphology, as shown in Fig. 6 (B). This complex core-shell structure can minimize the surface energy of mixed-oxide nanoparticles, which become more perfectly spherical with increasing shell $\mathrm{TiO}_{2}$ content. Apart from catalyst applications, this core-shell structure also shows great potential for utilization as high-quality, high-precision microelectronics and nanoelectronics over large-size wafers. Such fabrication of functional devices is elaborated upon in Section 4. 


\subsection{Synthesis of doped photocatalysts}

One way to improve the performance of semiconductor nanomaterials (i.e. $\mathrm{TiO}_{2}$ ) is to increase their optical activity by shifting the onset of the response from the UV to the visible region. This shift can be accomplished by narrowing the band gap through the doping with other elements, and thus altering the optoelectronic properties [3]. To obtain the reduced band gap energy, in contrast to the aforementioned separately supported structures, the dopants need to be homogeneously distributed in a crystalline parent semiconductor matrix without giving rise to a new crystallographic phase, as shown in Fig. 7(A) [105]. Additionally, the coupling collective oscillations of electrons in the conduction band of the doped metal nanoparticle surfaces to those in the conduction band of the support materials in the metal-support nanocomposites can improve the performance. The combination of semiconductor support and doped metal cluster has been reported to enhance photocatalytic activity by trapping the photo-induced charge carriers, thereby improving the charge transfer processes.

\subsection{1 $\mathrm{TiO}_{2}$-based photocatalysts}

As the most promising photocatalyst, $\mathrm{TiO}_{2}$ materials are expected to play an important role in addressing many environmental and energy challenges. Since the late 70's, a large body of research has discovered that $\mathrm{TiO}_{2}$ exhibits excellent catalytic properties for various reactions, and has also been used as support to dope various noble and transition metals for distinct catalytic applications. More importantly, nanophase $\mathrm{TiO}_{2}$, which has a large surface area that can facilitate a fast rate of surface reactions, is a widely used wide-bandgap semiconductor photocatalyst for a variety of solar-driven clean energy and environmental technologies [353]. However, the band gap of bulk $\mathrm{TiO}_{2}$ lies in the UV 
regime (3.0 eV for the rutile phase and $3.2 \mathrm{eV}$ for the anatase phase), partially restricting its application at low energy wavelengths, covering only a small fraction of the sun's energy $(<10 \%)$. Many methods have been reported to improve the photocatalytic activity of pure titania. Here four kinds of them that used in flame aerosol synthesis are listed together with the corresponding change of physicochemical properties of nanocatalysts.

In the first aspect, doping $\mathrm{TiO}_{2}$ with various transition metals during high-temperature flame synthesis can efficiently create impurity energy levels with the valence and conduction band of $\mathrm{TiO}_{2}$, and, therefore, facilitate the activation of $\mathrm{TiO}_{2}$ towards visible light [105]. As shown in Fig. 7(A), TEM images show indistinguishable crystalline morphology of pure and $\mathrm{Fe}$-doped $\mathrm{TiO}_{2}$ particles in a range of 9-20 nm, while the highly crystalline nature of these particles is evident from the distinct SAED crystallgraphic rings (Fig. 7 (A), right column) and HRTEM images (lattice fringes of 0.355, 0.343, and $0.324 \mathrm{~nm}$ spacing with respect to anatase, 4\% Fe-doped $\mathrm{TiO}_{2}$, and rutile). Then, as shown in Fig. 7 (B), the energy-filtered TEM (EFTEM) clearly displays the homogeneous distribution of Fe in the parent $\mathrm{TiO}_{2}$ matrix, which plays an important role in controlling the photoactivity of the doped nanocomposite. The homogenous distribution of the $\mathrm{Fe}^{3+}$-edge confirms the presence of vacant $3 \mathrm{~d}$-orbitals for trapping electrons during excitations. Therefore, the transition Fe lowers the band gap energy by introducing trap levels between the valance and conduction bands of $\mathrm{TiO}_{2}$.

The rate of oxalic acid mineralisation by $\mathrm{Fe} / \mathrm{TiO}_{2}(\mathrm{Fe} / \mathrm{Ti}=0.05)$ has been observed to be 6.4 times higher than that for similarly prepared bare $\mathrm{TiO}_{2}$ and Degussa P25 [104]. V-doped $\mathrm{TiO}_{2}$ showed that V doping enhances the photocalytic activity under both UV and visible light irradiation [364]. More recently, a series of semiconductor photocatalysts based on transition metals $\left(\mathrm{M}^{\prime}=\mathrm{V}, \mathrm{Cr}, \mathrm{Fe}, \mathrm{Co}, \mathrm{Mn}\right.$, 
$\mathrm{Mo}, \mathrm{Ni}, \mathrm{Cu}, \mathrm{Y}, \mathrm{Ce}$, and $\mathrm{Zr})$ incorporated into $\mathrm{TiO}_{2}\left(\mathrm{Ti} / \mathrm{M}^{\prime}=20\right.$ atomic ratio) materials have been synthesized by adopting a one-step FSP technique [102]. $\mathrm{H}_{2}$-TPR studies revealed the formation of Me-Ti bonds, suggesting the strong interaction of dopant metal- $\mathrm{TiO}_{2}$ in all the as-synthesized materials. The reduction peaks in $\mathrm{Cr}$-doped $\mathrm{TiO}_{2}$ shifted to much lower temperatures because of the increase in the reduction potential of titania and chromium. The existence of $\mathrm{Ti}^{3+}$ species with narrow band gap is highly beneficial to visible light-induced photocatalytic activity. The electrons migrate from the $\mathrm{TiO}_{2}$ nanoparticles to the chromium species, revealing a strong interaction between $\mathrm{Cr}$ and $\mathrm{TiO}_{2}$ nanostructure at the interface of the flame-made nanoparticles. Conversely, $\mathrm{Mn}^{3+}$ species combined with $\mathrm{TiO}_{2}$ because the surface metal dispersion was kept high after $\mathrm{TiO}_{2}$ loading. However, $\mathrm{Mn}^{3+}$ incorporated catalyst was inactive because of the small energy driving force for electrons to detrap from $\mathrm{Mn}^{2+}$. Among all of the catalysts tested, the transition metal $\left(\mathrm{M}^{\prime}=\mathrm{Cr}, \mathrm{Fe}\right.$, and $\left.\mathrm{V}\right)$ incorporated materials showed the most impressive catalytic performance in visible light. Cr-doped titania demonstrated superior catalytic performance, with the rate constant about 8-19 times higher than the rest of the metal doped catalysts.

In the second aspect, depositing noble metals on the surface of $\mathrm{TiO}_{2}$ (i.e. surface doping) can efficiently trap the excited state electrons and help efficient charge separation [370]. Teoh et al. [97] reported that $\mathrm{Pt} / \mathrm{TiO}_{2}$ photocatalysts favor a reductive pathway which is different and faster than the pathway followed when using Degussa P25 $\mathrm{TiO}_{2}$. Hydrogen evolution increases by one order of magnitude in water photo-splitting and by 30 times inmethanol photoreforming upon $1 \%$ gold addition on $\mathrm{TiO}_{2}$ [365]. Tiwari et al. [366] used an one-step flame aerosol reactor to synthesize 2-4 nm noble metal clusters-dispersed 30-40 nm titanium dioxide nanocomposites $\left(\mathrm{Pt} / \mathrm{TiO}_{2}, \mathrm{Pd} / \mathrm{TiO}_{2}\right.$, and bimetallic $\mathrm{Pt}-\mathrm{Pd} / \mathrm{TiO}_{2}$ ). On incorporation of the noble metal, the formation of the rutile phase of titanium dioxide 
was suppressed. Platinum particles dispersed on the $\mathrm{TiO}_{2}$ surface enhanced the photocatalytic activity compared to pristine $\mathrm{TiO}_{2}$. Optimum platinum loading for the highest photocatalytic activity was approximately $0.5-1.0 \%$ Pt. Bimetallic noble metal catalysts $\left(\mathrm{Pt}-\mathrm{Pd} / \mathrm{TiO}_{2}\right)$ showed enhanced photocatalytic activity compared to pristine titanium dioxide.

Thirdly, doping anion rather than metal ion can also narrow the band gap of $\mathrm{TiO}_{2}$. F-doped $\mathrm{TiO}_{2}$ exhibited the highest photocatalytic activity for the complete oxidation of acetaldehyde (ACE), surpassing benchmark Aeroxide $\mathrm{TiO}_{2}(\mathrm{P} 25)$, bare $\mathrm{TiO}_{2}$, and $\mathrm{Cu}$-doped $\mathrm{TiO}_{2}$ [367]. The lower proportion of native terminal hydroxyl groups, evaluated using high-field ${ }^{1} \mathrm{H}$ MAS NMR, on the particle surface of F-doped $\mathrm{TiO}_{2}$ possibly leads to a high photocatalystic performance.

In addition to doping with the metal ion or anionic, $\mathrm{TiO}_{2}$ nanoparticles coupling with other semiconductor can not only improve the efficient charge separation by trapping other semiconductor, but show good adsorption ability of pollutants as well. A combination of $\mathrm{WO}_{3}$ and $\mathrm{TiO}_{2}$, with bandgap of $2.8 \mathrm{eV}$ and $3.2 \mathrm{eV}$ respectively, allow the transfer of photogenerated electrons from $\mathrm{TiO}_{2}$ to $\mathrm{WO}_{3}$, which facilitate the effective charge separation. At the same time, the formation of a monolayer of $\mathrm{WO}_{\mathrm{x}}$ on $\mathrm{TiO}_{2}$ can increase the surface acidity and thus adsorb more hydroxyl groups and organic reactants on its surface. Akurati et al. [118] used FSP to obtain amorphous $\mathrm{WO}_{\mathrm{x}}$ species on the surface W-doped $\mathrm{TiO}_{2}$ substrate particles at the conditions of $<3.6 \mathrm{~mol}^{2} \mathrm{WO}_{3}$ concneration and high oxygen-to-fuel ratios. The formed amorphous layer enhances the acidity of the particles and then improves their photocatalytic activity for the degradation of methylene blue significantly compared to pure $\mathrm{TiO}_{2}$. 


\subsubsection{ZnO-based photocatalysts}

$\mathrm{ZnO}$, a wide-band gap semiconductor (with gap energy of about $3.30 \mathrm{eV}$ at room temperature), is of growing significance in photocatalyst substrate materials. In contrast to undoped $\mathrm{ZnO}, \mathrm{Au} / \mathrm{ZnO}$ showed a stronger absorption of visible light and a lower intensity of photoluminescence (PL) emission. Recombination of the photo-induced electron-hole pairs may be retarded due to the presence of discrete Au clusters by the shift in Fermi level and the stored photo-generated electrons in them. Height et al. reported synthesis of high surface area Ag-ZnO catalysts by FSP [169]. Silver metal clusters that discretely deposited on $\mathrm{ZnO}$ nanocrystals is believe to retard recombination of photoinduced electron-hole pairs. The Ag loading (1-5 at. \%) controlled the Ag cluster size from 5 to $25 \mathrm{~nm}$ but influenced little on the $\mathrm{ZnO}$ crystal size. The rate of photodegradation was optimal at an Ag loading 1-3 at. \%, because high loadings can decrease avaible particle surface for reactant adsorption and light absorption. The best photocatalytic performance was exhibited at the longest high-temperature residence times, as characterized by high crystallinity (XRD and UV-vis) compared with the wet-made $\mathrm{Ag}-\mathrm{ZnO}$ and reference $\mathrm{TiO}_{2}$ photocatalysts. $\mathrm{Au}-\mathrm{ZnO}$ and $\mathrm{Pt}-\mathrm{ZnO}$ nanocomposites have been synthesized by one-step FSP [170]. Although both flame-derived $\mathrm{Au}-\mathrm{ZnO}$ and Pt-ZnO possess similar physical properties (i.e., crystallite size and specific surface area), an improvement in photocatalytic degradation of methylene blue with respect to pure $\mathrm{ZnO}$ and commercial titania photocatalysts ( $\mathrm{JRC}^{-\mathrm{TiO}_{2}}$ and $\mathrm{P}-25$ ) was observed for $\mathrm{Au}-\mathrm{ZnO}$ but not for Pt-ZnO, probably because of the different type of Fermi-level equilibration between Au and Pt.

Before ending this section, we discuss the dopant-induced changes of physico-chemical properties 
of $\mathrm{TiO}_{2}$ - and $\mathrm{ZnO}$-based photocatalysts. It is widely recognized that photocatalytic activities strongly depend on many other factors, i.e. surface acidity, crystallinity, structure and so on [368]-[370]. In general, flame aerosol synthesis can produce a series of new photocatalysts with closely controlled morphology and composition (i.e. photocatalysts with various dopants, mixed metal oxide photocatalysts) due to a much wider choice of metal precursors. At the same time, this kind of rapid and high temperature continuous synthesis can yield photocatalysts exhibiting high intrinsic activity. However, the shorter residence time of flame aerosol synthesis results in lots of surface defects that have the detrimental effect of charge recombination and overwhelm the benefits of surface energetics. Therefore, how to design flame-made photocatalysts overcoming the inherent charge trap defects and recombination centres and explore the potentiall efficient photocatalysts still reminds a significant challenge.

\subsection{Synthesis of nanophosphor materials}

More recently, nanophosphor materials have attracted substantial consideration in display fields because of their improved properties such as high luminescent efficiency, quantum effect, and higher doping concentration without concentration quenching. As such, they have potential applications in biolabeling, biosensors, and multiphoton confocal microscopy, along with promising usage as "invisible" infrared materials. Flame synthesis has been demonstrated to be a single-step, continuous, and high-rate synthesis method for non-agglomerated upconversion nanophosphors [88],[181]-[193],[368]-[373]. The heat released from the combustion of a gaseous or liquid fuel and the precursor itself can provide the high-temperature environment, which is favorable to phosphor synthesis. 
Moreover, flame temperature, particle residence time, and particle size determine the characteristics of the phosphor materials (i.e. enhanced display luminescence and resolution).

Rare-earth (RE) doped upconversion nanophosphors, possessing sharp luminescence in the visible range with near-infrared excitation, is of great interest [181]-[193]. For example, europium-doped yttrium oxide $\left(\mathrm{Y}_{2} \mathrm{O}_{3}: \mathrm{Eu}^{3+}\right)$, is a remarkable red phosphor, which has been widely used in optical displays and lighting applications. Under ultraviolet (UV) excitation, $\mathrm{Y}_{2} \mathrm{O}_{3}: \mathrm{Eu}^{3+}$ gives a sharp red emission line at $611 \mathrm{~nm}$, and the luminescence efficiency is high because its charge transfer transition is located in the UV range. Nanoscale $\mathrm{Y}_{2} \mathrm{O}_{3}: \mathrm{Eu}^{3+}$ phosphor showed significant promise in high-resolution displays (field emission display, flat panel display, thin film electroluminescence panel, etc.) because the quantum efficiency of doped nanocrystals increases as the particle size decreases [185]. $\mathrm{Y}_{2} \mathrm{O}_{3}: \mathrm{Eu}^{3+}$ also showed the nanophosphor peak in the red region of the visible spectrum with a maximum peak at a wavelength of $609 \mathrm{~nm}$ when excited with $398 \mathrm{~nm}$ photons. The cubic nanoparticles exhibited longer decay times but lower maximum photoluminescence intensity than did commercial micron-sized bulk $\mathrm{Y}_{2} \mathrm{O}_{3}: \mathrm{Eu}^{3+}$ phosphor powder [183]. As far as the structures of nanophosphor materials are concerned, it greatly depends on their sizes. Dosev et al. reported flame-made nanoparticles that were separated by centrifugation into two size groups, a smaller one of 5-60 nm and a large one of 50-200 nm. The fluorescence spectra, the electron diffraction pattern, and the XRD pattern of the large group particles were typical of the stable cubic $\left(\mathrm{Mn}_{2} \mathrm{O}_{3}\right.$ type) phase of bulk $\mathrm{Y}_{2} \mathrm{O}_{3}$, whereas those of the small group particles were quite different and indicated the possible presence of higher density metastable mixed phases including monoclinic, with some indication of a face-centered cubic phase [182].

Interestingly, it is noted that $\mathrm{Y}_{2} \mathrm{O}_{3}: \mathrm{Eu}^{3+}$ or $\mathrm{SiO}_{2}: \mathrm{Y}_{2} \mathrm{O}_{3}: \mathrm{Eu}^{3+}$ phosphor particles can be enhanced 
much more by forming a complex core-shell structure, i.e., phosphorous core and silica shell [189]. First, the silica shell could reduce the reflectance of exciting radiation compared to pure phosphor particles. Second, the silica shell can stabilize the surface of the particles that leads to the luminescence enhancement of the core phosphor. Third, formation of the core shell structure provides additional properties such as hydrophilization and detoxification for the phosphor core. The $\mathrm{Y}_{2} \mathrm{Si}_{2} \mathrm{O}_{7}: \mathrm{Eu} / \mathrm{SiO}_{2}$ core-shell phosphor particles, as shown in Fig. 6(C), were successfully synthesized by one-step FSP using a precursor aerosol containing yttrium nitrate, europium nitrate, and colloidal silica nano-particles [189]. Similar to the strategy of making core-shell structures in Section 3.1.6, high flame temperature enabled the formation of the core-shell structure, whereas the low temperature did not. In addition, high silicon concentration in the precursor solution led to the desired ideal spherical core-shell structure shown in Fig. 6(C), instead of non-spherical core-shell structures. Under ultraviolet excitation, the ideal spherical core-shell particles emitted the best photoluminescence.

There have been many attempts for flame synthesis of other kinds of rare-earth doped nanophosphors. Cerium-doped $\mathrm{Y}_{3} \mathrm{Al}_{5} \mathrm{O}_{12}\left(\mathrm{YAG}: \mathrm{Ce}^{3+}\right.$ ) particles, as yellow emission phosphor, are another remarkable phosphor in the solid-state lighting field, since it can be used to generate white light by mixing with blue light emitting diodes. The optimum cerium-doping concentration of YAG:Ce nanoparticles is 4.0 at.\%, achieving a quantum efficiency of $45.0 \%$ [372]. The efficient emission of YAG:Ce nanoparticles also originates from a relatively good distribution of Ce ions incorporated into the host material of YAG, as evidenced from elemental mapping analysis. A more complex rare-earth doping method in flame synthesis is needed to obtain the desired blue, green, or red emissions, respectively [368]. For example, $\mathrm{Y}_{2} \mathrm{O}_{3}:(4 \% \mathrm{Yb}+1 \% \mathrm{Tm})$ nanocrystals exhibit blue emissions between 450 
and $500 \mathrm{~nm}$, in which the strongest luminescence peak at $477 \mathrm{~nm}$ is consistent with the ${ }^{1} G_{4} \rightarrow{ }^{3} H_{6}$ transition of $\mathrm{Tm}^{3+}$ that requires three-step energy transfer from $\mathrm{Yb}^{3+}$ ions. The $\mathrm{Y}_{2} \mathrm{O}_{3}:(4 \% \mathrm{Yb}+1 \% \mathrm{Ho})$ nanocrystals have green emissions near $543 \mathrm{~nm}$ and red emissions near $665 \mathrm{~nm}$, which are identified as transitions of $\left({ }^{5} S_{2},{ }^{5} F_{4}\right) \rightarrow{ }^{5} I_{8}$ and ${ }^{5} F_{5} \rightarrow{ }^{5} I_{8}$ of $\mathrm{Ho}^{3+}$, respectively. $\mathrm{Y}_{2} \mathrm{O}_{3}:(8 \% \mathrm{Yb}+6 \%$ Er) nanophosphors have strong red emission peaks at 657,663 , and $673 \mathrm{~nm}$, which are attributed to the ${ }^{4} F_{9 / 2} \rightarrow{ }^{4} I_{15 / 2}$ transition of $\mathrm{Er}^{3+}$. Besides the above yttria-based nanophosphors, Mn-doped zinc silicate is widely used as a green phosphor in plasma display panels and cathode ray tubes because of its chemical stability, high luminescence efficiency, and semiconducting properties. The luminescence properties of flame-made $\mathrm{Zn}_{2} \mathrm{SiO}_{4}: \mathrm{Mn}^{2+}$ particles were found to improve at higher methane flow rates during pyrolysis and at higher annealing temperatures [81].

\subsection{Doping diagram for nanocomposite synthesis}

To obtain the designed structures of nanocomposites, i.e., supported, core-shell, or homogeneously-uniform ones, as mentioned above, a doping diagram for various nanocomposite syntheses is presented here to meet the different functional utilizations of flame-made materials. In fact, the attempt for drawing such a diagram can be traced back to the early work of Ehrman, Friedlander, and Zachariah in 1998 [270].

Here, three determining factors are summarized, including precursor reaction type, material miscibility, and feasible burner design, abbreviated as "RMB". Five important nanocomposite structures are then categorized according to the "RMB" design criteria, as illustrated in Fig. 8. The ordinate represents the miscibility degree of binary metal oxide components, whereas the abscissa indicates their 
doping status, i.e., inter-particle or intra-partible doping. The intra-particle doping, where atom diffusion plays a key role, is easily enhanced by some special conditions of higher temperature, smaller sizes of coalesced particles of multi-components, etc, and vice versa for inter-particle doping. From left to right, the supported structure of nanocomposites to produce metal-support catalysts is displayed, as described in section 3.1. A recent example of the supported structure can be found in the work of Li and co-workers [324]. In this work, the titania supported palladium nano-catalysts $\left(\mathrm{Pd} / \mathrm{TiO}_{2}\right)$ were synthesized in a FASP process subject to the stagnation-flow configuration, in which the palladium clusters are well dispersed on $\mathrm{TiO}_{2}$ support. Supported structure is usually favored in a low temperature synthesis environment (short residence time and high quenching rate) to hinder the severe sintering and intra-particle doping of oxide components. In terms of the precursor reaction and burner desgin, two possible synthesis routes are available. When the reactions of the precursors are temperature driven, the synthesis process is insensitive to the burner type as long as the two components have large difference in characteristic reaction time, nucleating and growing in sequence, which results in a pattern of inter-particle doping. When the precursors are sensitive to both temperature and radicals, i.e., one component is temperature driven and another component is radical driven, the premixed flame burner is advantageous, as it can provide abundant radicals (like $\mathrm{OH}$ ) at the early flame stage to initiate fast decomposition of the radically driven component. For miscibility, recent research claims that the miscibility at the nano-scale is not absolute and may be different than that for bulk materials. Complex metal-support interactions are found to exist between the components [112].

The segregated structure of the nanocomposites is composed of separate particles of each component mixed together. Two possible synthesis routes are shown in the diagram in Fig. 8. If the 
decomposition of both components are temperature driven, either type of burner can be used. If the decomposition is driven by radicals, the premixed flame burner is preferred to promote inter-particle doping. Notably, the segregated structure can also be conveniently obtained by employing two nozzle flames together for ensuring inter-particle doping, which can be found in the work of Strobel et al. [26], for the case of $\mathrm{BaCO}_{3} / \mathrm{Al}_{2} \mathrm{O}_{3}$ particles.

Similar to the supported structure, the core-shell structure shares the similar synthesis routes, but with a significant difference in the high temperature condition. The typical examples are those mentioned earlier by Feng et al. [12] (Section 3.1.6) and Hasegawa et al. [189]. In the former, the high $2500^{\circ} \mathrm{C}$ flame temperature causes the (molten) $\mathrm{TiO}_{2}$ shell to encapsulate the inner $\mathrm{CeO}_{2}$ core, accommodating a spherical morphology as shown in Fig. 6(B). In the latter, the $\mathrm{Y}_{2} \mathrm{Si}_{2} \mathrm{O}_{7}$ : Eu core and the $\mathrm{SiO}_{2}$ shell structure are favored by a higher flame temperature up to $3052 \mathrm{~K}$, in which the formation of a perfectly spherical core-shell structure requires a high concentration of $\mathrm{SiO}_{2}$ in the precursor.

In terms of intra-particle doping, there are another two important structures of nanocomposites, i.e., the Janus particle and the uniform particle. The Janus particle is a spherical particle consisting of two distinct parts with different chemical compositions that can hold both the chemical and physical properties of two phases, for example, $\mathrm{Fe}_{2} \mathrm{O}_{3} / \mathrm{SiO}_{2}$ as reported in the literature by Zhao and Gao [373]. The uniform particle, on the contrary, is a spherical particle that consists of uniform multi-components (like the aforementioned perovskites, spinel, $\mathrm{Ce}_{x} \mathrm{Zr}_{1-x} \mathrm{O}_{2}$, transition-metal doped $\mathrm{TiO}_{2}$, etc.). As shown in the diagram, the homogeneously-uniform particles can be synthesized in two typical routes. For the case when the decompositions of both components are temperature driven, either type of burner can be used as long as the flame temperature is sufficiently high for particles to coagulate and coalesce into a single 
sphere under fast quenching. When the radically driven decomposition reactions are involved in the synthesis, as mentioned above, the premixed flame burner is advantageous over the diffusion flame burner for a high radical concentration to facilitate the reactions. The Janus particle synthesis is rarely reported in flame aerosol synthesis. The work reported in the literature by Zhao and Gao [373] adopts ferric triacetylacetonate (pyrolysis) and tetraethylorthosilicate (hyrolysis) as precursors, and the iron and silicon components formed an intermixed solid, which then undergoes phase separation process in the flame.

\section{Flame aerosol synthesis of carbon-metal oxide nanocomposites}

More recently, to meet the ever-increasing demand for renewable energy resources and, in particular energy storage devices, carbon-metal oxide (CMO) nanocomposites have received considerable attention in various kinds of applications, such as supercapacitors and Li-ion batteries, since they not only combine the properties of carbon nanomaterials and nanostructured metal oxides but also hold new extraordinary properties caused by the interactions between them [374][375][376]. Because of their low cost and good corrosion resistance, carbon materials are widely used as electrodes in most commercial supercapacitors. However, the low energy density of carbon-based supercapacitors (3-5 $\left.\mathrm{W} \cdot \mathrm{h} \mathrm{kg}^{-1}\right)$, which is dramatically lower than electrochemical batteries (or even lead acid batteries) by almost an order of magnitude, limits their application in energy storage devices for vehicles or solar power units [374]. To improve the energy density, as well as the specific capacitance, of carbon-based electrodes, transition metal oxides, including $\mathrm{TiO}_{2}, \mathrm{~V}_{2} \mathrm{O}_{5}, \mathrm{Mn}_{3} \mathrm{O}_{4}, \mathrm{Fe}_{2} \mathrm{O}_{3}, \mathrm{Co}_{3} \mathrm{O}_{4}, \mathrm{NiO}, \mathrm{CuO}, \mathrm{ZnO}, \mathrm{RuO}_{2}, \mathrm{SnO}_{2}$, and $\mathrm{WO}_{3}$, have been attempted to be deposited on carbon materials to make CMO nanocomposites, after the pioneering work of transition metal oxides as anode materials [377]. So far the demonstrated synthesis 
method of CMO nanocomposite mainly include: (1) hydrothermal, solvothermal, photochemical or chemical syntheses; (2) chemical vapor deposition, electrodeposition, electrochemical deposition; (3) microwave-assisted, or ultrasound-assisted syntheses; and others. Synthesizing various kinds of dimensional (0-D, 1-D, 2-D, and 3-D) carbon nanostructures enable us to tailor flexibly the properties of the CMO nanocomposites.

As aforementioned, flame aerosol synthesis has a demonstrated history of scalability and offers the potential for high-volume commercial production, at reduced costs, for synthesis of either metal oxide nanoparticles or commercial carbon black materials. However, studies on flame aerosol synthesis of carbon-metal oxide nanocomposites are surprisingly scarce, despite their promise and need in applications involving energy storage and battery materials. A number of studies on flame synthesis of carbon nanotubes and other carbon nanostructures have been reviewed, including a recent paper in PECS [17]. Therefore, in this section, from the point of view of carbon nanostructures, we first provide a brief review on flame "aerosol" synthesis of carbon nanomaterials (governed by the aerosol nucleation/coagulation route given in Fig. 1) mainly from the combustion community. Then, a prospect of the scalable synthesis of CMO nanocomposites by flame aerosol synthesis is discussed.

\subsection{Various carbon nanostructures from flame aerosol synthesis}

For carbon-based nanoproducts, the flame aerosol synthesis route is especially well suited, possessing an intrinsically cheaper source of process heat than any other technique, since the hydrocarbon gas provides both the enthalpy and reagent needed for synthesis. Therefore, it is already widely used to produce commercial products in tonnage quantities such as carbon black. 


\subsubsection{Fullerenes (0-D)}

The entangled relationship between soot and fullerenic nanostructures formations in flames has been shown in work demonstrating the synthesis of fullerenes and CNTs in sooting flames. Much of the first work on fullerene synthesis in flames were done by Homann and co-workers [378][379] and Howard and co-workers [380][381][382] using premixed benzene/oxygen flames, with and without inert diluent gas, at low-pressures ( $<40$ torr). Later, experiments [383] were conducted using diffusion flames.

In the premixed configuration, Howard and co-workers [381] investigated burner-stabilized laminar flat flames (McKenna burner in Fig. 2b). Samples of condensable flame species, including PAHs, fullerenes, and soot, were collected a different distance from the burner through a quartz probe, and analyzed by high-performance liquid chromatography to determine the yields of fullerenes. They observed a "first" formation region of fullerenes early in the flame, occurring simultaneously with a large rate of $\mathrm{PAH}$ consumption, suggesting that the route of fullerenes formation involves reactions of PAH. The observed "second" region, which occurred later in the flame, accounted for most of the fullerenes mass produced, implying a route more consistent with stepwise acetylene addition mechanism. Nonetheless, they found that in both regions, fullerenes consumption involved reactions between fullerenes and soot. Additionally, measurements indicated that the different PAHs grew roughly simultaneously, reached a peak concentration, and decayed together in a consumption process that occurred concurrently with a rapid rise in soot mass concentration early in the flame, representing a 
behavior consistent with the major soot formation being from PAH and occurring along with the minor fullerenes formation in their flame.

In the same configuration, Homann and co-workers [379] noticed the dependence of fullerenes and soot production on temperature. They detected a new class of "particles," named aromers (due to their structural properties), in flames existing prior to both fullerenes and soot particles. Arguing that the products of these aromers are determined by the flame temperature and the concentration of growth species such as acetylene, they found that at high temperatures and with relatively low concentrations of acetylene, these aromers formed fullerenes, while at low temperatures and/or high concentrations of small growth species, mainly soot was formed.

On the other hand, in the diffusion flame configuration, Howard and co-workers [383] found the maximum flame temperature to be of minor importance in fullerene formation; instead, residence time was the key parameter. They employed a low-pressure laminar jet flame, with argon-diluted benzene flowing within a co-flow of oxygen (as shown in Fig. 2a), and analyzed samples of condensable material at axial distances along the flame. The highest concentration of fullerenes was always detected just above the visible stoichiometric surface of a flame. Moreover, the percentage of fullerenes in the condensable material increased with decreasing pressure, with the overall highest amount of fullerenes associated with a surprisingly high dilution of fuel with argon. These effects were attributed to flame length (and hence residence time), where a shorter flame, resulting from high dilution or lower pressure, favors the formation of fullerenes rather than soot. At the same time, the amount of soot and precursors of both soot and fullerenes were reduced, indicating a stronger correlation of fullerene consumption to the total amount of soot than of fullerene formation to precursor concentration. The HRTEM analysis of 
the soot showed an increase of the curvature of the carbon layers, and hence increased fullerenic character, with increasing distance from the burner up to the point of maximum fullerene concentration. After this maximum, where soot and fullerenes were consumed by oxidation, the curvature decreased. In addition to the soot, the samples included fullerenic nanostructures such as tubes and spheroids including highly-ordered multi-layered or onion-like structures. The soot itself showed highly ordered regions of ongoing fullerenic nanostructure formation.

\subsubsection{Carbon nanotubes (1-D)}

The carbon nanotube (CNT), possessing 1-D nanostructure, has relatively smaller surface area as compared with the 0-D fullerene. However, the high conductivity of 1-D nanostructures are found to be greatly helpful to the redox activities of transition metal oxides, resulting in much higher rate capability. With respect to flame aerosol synthesis, earlier in 1994, Duan and McKinnon [384] observed CNTs randomly distributed in the soot in rich benzene-air premixed flames at low pressure. In 1995, Chowdhury et al [385] studied low-pressure benzene, acetylene, and ethylene premixed flames, finding the presence of various fullerene nanoparticles and some CNTs on collection probes.

Later, metal catalyst nanoparticles were specifically seeded into sooting flames to synthesize carbon nanotubes (CNTs). Several models have been proposed to explain CNT formation, such as the Baker growth mechanism [386], the vapor-liquid-solid mechanism [387], and the yarmulke (skull-cap) mechanism [388]. What is generally observed is that carbon precursors, e.g. alkane, alkene, alcohol, or $\mathrm{CO}$, decompose on transition metal nanoparticles, and can precipitate on one side of the nanoparticles, forming CNTs [389]. However, if the CNT growth conditions are not optimal, carbon can completely 
encapsulate the nanoparticles, deactivating them, which is the most likely scenario [390]. As such, in the flame aerosol route, synthesis of catalyst nanoparticles is concurrent with growth of CNTs, under the right conditions. For example in the Yarmulke or "skullcap" mechanism, a metallic catalytic particle removes hydrogen atoms from adsorbed hydrocarbons, liberating carbon to diffuse along the length and attach to the open edges of the forming nanotube [388]. The shape of the "cap" comes from the newly-arriving carbon atoms forming the carbon lattice that follows the spherical shape of the catalyst particle [390][391]. Possibility exists for a second 'cap,' as well as additional ones, to form above the first one surrounding the catalyst, allowing more layers of carbon to form, thus resulting in the growth of multi-wall nanotubes (MWNTs). Nonetheless, in the Yarmulke mechanism, the diameter of the nanotube is governed by the size of the catalyst particle, explaining the often found correlation between particle size and CNT diameter in the literature [392][393]. It has been furthermore observed by Vander Wal and Hall [392] that $1 \mathrm{~nm}$ diameter Fe catalytic particles formed single-wall carbon nanotubes (SWNTs), while 1 to $10 \mathrm{~nm}$ catalyst particles formed MWNTs.

Earlier, Diener et al. [394] had achieved synthesis of SWNTs at reduced pressures in fuel-rich acetylene and ethylene surface-mixing flames when metallocenes were added. While no SWNTs could be synthesized using benzene as fuel, long metal-filled MWNTs with many defects were abundant. Their experimental results suggested that the best fuels for combustion synthesis of fullerenes are the worst for combustion synthesis of SWNTs, and vice-versa. Acetylene flames were barely capable of producing fullerenes, while indene flames had even higher fullerene yields than benzene flames. Since a suggested route of fullerenes formation in flames proceeds from a phenyl ring through PAHs, which knit together aromers [379] and/or grow by acetylene addition [395] and (in the correct pressure and 
temperature range) finally rearrange to become a fullerene [396], uncombusted benzene seeds PAH formation, jump-starting fullerene formation. In contrast, the growth from acetylene into a phenyl ring occurs relatively slowly in comparison to ring growth reactions. They also suggested that it may be possible to produce SWNTs in an acetylene flame without a lot of soot byproduct, if all of the acetylenic carbon can be converted to SWNTs before soot formation is initiated.

As mentioned above, CNTs can be produced in non-sooting flames. Vander Wal and co-workers [397] demonstrated synthesis of SWNTs at atmospheric pressure via a simple laboratory-scale diffusion flame, using different hydrocarbon reagents and inert dilution. They observed that dilution of the fuel stream with an inert gas, either nitrogen or argon, was critical to CNT synthesis. Absence of the inert carrier resulted in soot formation and the complete encapsulation of the metal nanoparticles within a carbonaceous matrix with no CNTs being formed. They argued that the net effect of an inert additive is to decrease the rates of PAH formation and soot growth reactions, both of which deplete the hydrocarbon reagent pool and produce species/compounds that deactivate the catalyst particles. They proposed that dissociative adsorption is likely the first step in the growth of the SWNTs, given an active metal catalyst particle. Since $\mathrm{C}_{2}$ species readily undergo dissociative adsorption, acetylene becomes essential to the molecular growth path of the CNT.

On the other hand, without catalysts, Merchan-Merchan et al [398] found that MWNTs were produced in a counter-flow oxy-methane diffusion flame on the fuel-rich side of the flame front at an oxygen enrichment of 50\% (shown in Fig. 2c). The MWNTs were mixed with soot aggregates and carbon nanoclusters. They gave a phenomenological explanation of CNT formation through pyrolysis of hydrocarbons resulting from an increase of radicals due to oxygen enrichment. Such non-catalytic 
growth of CNTs has long been postulated, especially in flames where very high temperatures can be achieved.

With the aerosol route, long SWNTs are also achievable. Unrau et al. [399] utilized an ethylene-oxygen inverse diffusion flame seeded with ferrocene to produce and collect SWNTs 1-3 nm in diameter and $200 \mathrm{~nm}$ to $1 \mu \mathrm{m}$ in length.

Many researchers have studied premixed flames to synthesize CNTs. Vander Wal and co-workers [400][401] used premixed co-flow flames to grow SWNTs by seeding the fuel line with ferrocene and compositions of metal nitrates serving as catalyst precursors. Ferrocene-generated Fe nanoparticles were able to produce bundles of self-assembled SWNT with diameters as small as two nanometers. In one configuration (i.e. pyrolysis flame) [400], the catalyst precursor aerosol was supplied through the metal tube inside of the flame, where there is no combustion, but there is enough heat supplied by the reaction outside of the tube for nanotube formation to occur. In some cases, acetylene flames with Fe and $\mathrm{Ni}$ [400][402] catalysts resulted in significant yields of SWNTs [400][401][402] and MWNTs [402]. These results showed that the size of the metal catalyst directly influenced the size of the CNT. Moreover, as the diameter of the catalyst particle increased, the yield of SWNTs decreased, and their length became shorter [400]. Another interesting observation was that temperature played a negligible role in CNT growth from Fe particles.

However, Vander Wal and Ticich [403] also found that a catalyst particle can initially be larger than the subsequent CNT. Yet, for that to happen, the particle would have to be broken up in the flame so that the smaller catalyst fragments can facilitate CNT growth. Another observation was that the increase of CO resulted in increased encapsulation of CNTs by amorphous carbon. Fe was noted to lead to higher 
CNT yield than that for Ni. A further study suggested that Fe catalyst might be more appropriate for SWNT growth, while Ni catalyst might be better used for nanofiber growth [392]. They suggested that Fe particles are catalytically active at smaller diameters than $\mathrm{Ni}$, thus resulting in the growth of smaller diameter structures, such as SWNTs.

Height and co-workers [404] also utilized premixed co-flow flames to grow SWNTs. A detailed characterization of SWNT growth was given with emphasis on flame position and air-to-fuel ratio. Goel et al. [405] compared premixed and diffusion flames for benzene/oxygen flames. They found that CNT yield was more favorable in diffusion flames than in premixed flames, for their setups. CNTs were only observed in the premixed flame when the residence time was increased considerably higher than that of the diffusion flame counterpart.

Height et al. [406] utilized an acetylene flame with vaporized $\mathrm{Fe}(\mathrm{CO})_{5}$ acting as a catalyst precursor to generate SWNTs. Nanotubes were collected using a thermophoretic sampling probe at various positions above the flame at equivalence ratios ranging from 1.5 to 1.9 and flame temperatures from 1500 to $1800 \mathrm{~K}$. Discrete carbon particles were found to be dominant at the lower equivalence ratios, while soot began to contaminate CNT yield closer to $\Phi=1.9$.

Tse's research group [407] examined rich ethylene-air premixed flames in divergence-stabilized stagnation-point geometry (shown in Fig. 2-f.1), seeded with vaporized ferrocene $\mathrm{Fe}\left(\mathrm{C}_{5} \mathrm{H}_{5}\right)_{2}$, to deposit CNTs on a substrate. The equivalence ratios were below the threshold for sooting. Short aspect ratio MWNTs, similar to those found by Merchan-Merchan et al. [398], were deposited on the substrate, despite short residence times. Longer carbon nanofibers were grown in methane-air flames, but no CNTs. 


\subsubsection{Graphene flakes (2-D)}

To the best knowledge of the authors, there have been no available publications on the flame aerosol synthesis of graphene flakes. Although not flame synthesis, Fenklach and co-workers [408][409] synthesized graphene-flakes using atmospheric-pressure microwave plasmas injected with liquid ethanol droplets, paving the way for the gas-phase route. By flame aerosol synthesis, the closest research is by Minutolo et al. [410], who produced two-dimensional carbon nanostructures using an atmospheric pressure ethylene/air laminar premixed flame, stabilized on a McKenna burner, with an equivalent ratio of 1.83. The collected samples revealed a network of aromatic compounds connected by non-aromatic bonding to form atomically-thin disk-like structures. The demonstration of such gas-phase synthesis has implications for large-scale production of graphene, along with the possibility that flame synthesis may also achieve such results, presenting an area ripe for research.

Interestingly, flame aerosol synthesis differs from another typical flame synthesis method, flame-assisted CVD-type process, in that the residence time in flame aerosol reactor is significantly higher than the precursor chemical reaction time $\left(\tau_{r e s}<<\tau_{c h e m}\right)$, and vice versa, as seen detail in Section 4.1. Tse and co-worker developed a multi-element inverse-diffusion-flame burner, in which the radially-uniform post-flame gas species are deposited on a substrate. By using this novel flame-assisted CVD method, it was demonstrated that few-layer graphene can be grown on copper and nickel substrates in open environments at high rates [291]. 


\subsubsection{Nanodiamond (3-D)}

Although scalable flame aerosol synthesis of nanodiamond (ND) would be extremely-appealing, there seems to be limited studies in the area, particularly compared with dozens of studies on flame-assisted CVD of diamond film. Since the 1980s, combustion-related synthesis of diamond particles has been accomplished by the detonation of explosives with a negative oxygen balance in steel containers under vacuum conditions [411][412][413]. Although nanodiamond synthesis by explosive detonation is still widely employed, the process is batch-to-batch and not continuous, with purification processes needed.

One of the few flame-based synthesis of nanodiamond was accomplished by Frenklach and co-workers [414]. Cubic and hexagonal diamond nanoparticles $(10-500 \mathrm{~nm}$ in diameter) were created by microwave-assisted low-pressure $(<150$ Torr $)$ combustion of acetylene in oxygen, in both premixed and diffusion flames. Lower reactor pressure and higher $\mathrm{C}$ to $\mathrm{O}$ atomic ratios resulted in larger particle sizes. Only $\mathrm{C}$ to $\mathrm{O}$ atomic ratios between 0.83 and 1.0 produced crystalline diamond nanoparticles, with other ratios yielding graphite, soot, and amorphous carbon phases. Again, this should be a fertile research area with regards to the scalable production of ND.

\subsection{Flame aerosol synthesis of carbon-metal composites}

Carbon can be readily doped into metal-oxide nanoparticles. For example, using a low-pressure acetylene-oxygen premixed flame injected with TTIP, Chen et al. [425] synthesized carbon-doped titania nanopowders, which showed visible-light photocatalytic ability. They suggested that carbon species associated with catalytic sites on the anatase surface allow carriers generated by photon 
absorption to transfer quickly onto catalytic sites and perform the subsequent catalytic reactions. Similarly, Al-Sharab et al. [426] have also synthesized carbon-doped titania nanoparticles using low pressure premixed flames, for applications in solar cells. The rapid development of flame spray pyrolysis (FSP) or flame-assisted spray pyrolysis (FASP) techniques in the past two decades enable one-step aerosol synthesis of carbon-metal oxide nanocomposites. Ko et al. [375] demonstrated a novel FSP-type fabrication of carbon-manganese oxide $\left(\right.$ Carbon- $\left.\mathrm{Mn}_{3} \mathrm{O}_{4}\right)$ composite with open nanochannels, and then characterized their electrochemical performance as anode materials for lithium ion batteries. The aqueous spray solution was prepared by dissolving $0.2 \mathrm{M}$ manganese acetate (as can be found in Appendix Table 1) and 0.5 M sucrose in distilled water, where the sucrose served as the carbon source. Subsequently, polystyrene nanobeads were added to the clear solution, in a weight ratio of 1.55:1 with respect to $\mathrm{Mn}_{3} \mathrm{O}_{4}$. As shown in Fig. 9, the amorphous-like $\mathrm{Mn}_{3} \mathrm{O}_{4}$ nanoparticles are embedded in a carbon matrix with three-dimensional channels. The nucleation and crystal growth process occurs in the rear part of the high-temperature diffusion flame $\left(\sim 2500{ }^{\circ} \mathrm{C}\right)$, producing $\mathrm{Mn}_{3} \mathrm{O}_{4}$ nanopowders, with a mean size around $42 \mathrm{~nm}$, as deduced from TEM images shown in Fig. 9(a). For carbon-free $\mathrm{Mn}_{3} \mathrm{O}_{4}$ nanopowders, the high resolution TEM image as shown in Fig. 9(b), reveals the single crystalline structure of the $\mathrm{Mn}_{3} \mathrm{O}_{4}$, whereas the dot-mapping images, as shown in Fig. 9(c), show the carbon-free $\mathrm{Mn}_{3} \mathrm{O}_{4}$ nanopowders, indicating the well-dispersed nature of $\mathrm{Mn}_{3} \mathrm{O}_{4}$ nanoparticles in the carbon matrix. This kind of structure may lead to the design of compact energy storage devices.

Interestingly, using hot-wall heating instead of flame, Zachariah and co-workers [376] developed an aerosol spray pyrolysis process to synthesize homogeneously unique amorphous carbon-MnOx nanocomposite particles, as shown in Fig. 10 (a)-(b). Such a structure effectively accelerates the 
conversion reaction, preventing the agglomeration of $\mathrm{Mn}$ metal grains during the conversion reaction with $\mathrm{Li}$, thus significantly improving cyclability. Generally, the unique structure of the amorphous carbon-MnOx nanoparticles can provide the best anode performance for manganese oxide to date, implying FSP can be a very promising technique for electrode material manufacture.

As for other kinds of carbon-metal oxide structures, metal-oxide nanoparticles have been coated on the surface of carbon nanofibers to form typical core-shell structures, in which the nanofiber not only serves as the physical backbone support but also offers the charge transport channel, while the metal oxide improves the specific capacitance and energy density [374]. However, such nanostructures have yet not to be reported using flame aerosol synthesis. The scalable flame aerosol synthesis of 2-D carbon nanostructure (like graphene) supporting metal oxides are of tremendous desirability. 2-D graphene possesses high surface area, high electronic conductivity, and high mechanical strength, which are greatly beneficial to the improvement in the charge/discharge kinetics of supercapacitor and battery electrodes. Addressing flame aerosol synthesis to produce such kinds of functional structures is both an opportunity and a challenge to investigators in the field. Indeed, future combination of flame aerosol synthesis and flame-assisted CVD may be quite worthwhile.

\section{Fabrication of functional nanostructured films and devices by flame aerosol deposition}

Thin micropatterned metal-oxide functional films are of great relevance for a variety of applications, such as gas sensors, solar cells, fuel cells, and catalytic reactors, in energy and environment, material bio-interfaces, microelectromechanical systems (MEMS), drug delivery, and medical diagnostics. The fabrication of these films and devices is usually achieved through self-assembly of nanoparticles in gas- 
or liquid-phase processes or through compaction of flame-made nanopowders. In this review, we only focus on gas sensing and photovoltaic applications, which are suitable topics for energy and environmental concerns. The well-established processing routes for preparing ceramic thin films include colloidal-based wet coating processes, e.g., spin coating, slip coating, dip coating, etc., and dry aerosol-based particle deposition, e.g. electrospray deposition, plasma spraying deposition, and flame aerosol deposition (FAD) [9][427]. Wet-based processes require time intensive and often complex procedures to reduce capillary stresses or particle agglomeration, along with environmental concerns of liquid byproducts [427]. In contrast, dry processes not only exclude these disadvantages because of the direct deposition of particles from the gas phase, but also are much simpler and require fewer unit operations. Comparatively, electrospray deposition is generally constrained in its throughput by charging effects, and plasma spray deposition has relatively high energy consumption. As a result, flame aerosol deposition exists as one of most economically scalable means for the fabrication of functional films and devices.

Based on the feeding state of precursors, the flame aerosol deposition method includes two routes, FSP-based and VAFS-based ones. The former is established by placing a substrate downstream of the flame spray pyrolysis burner, as shown in Fig. 2.g; while the latter is formed by placing a substrate downstream of the premixed stagnation flame (PSF) burner, as shown in Fig. 2.f (1-4). However, despite of many studies on flame aerosol deposition in the past ten years [9][10],[213]-[224],[311]-[322], the underlying physics governing the deposition and assembly mechanisms, as well as the core technology dominating industrial operation and product specifications, are still not very well understood or explored. In this review, one of the goals is to extract important "problems" from previous studies so that they can 
be appropriated studied in the near future.

\subsection{Controlling diagram of various deposition regimes: CVD vs. FAD}

To begin with, we shall distinguish the differences between flame aerosol deposition and the well-known commercial chemical vapor deposition of materials science. Based on experimental findings, Biswas and co-workers summarized three deposition and film growth mechanisms: $(i)$ vapor dominant regime (basically CVD producing dense films), (ii) vapor-particle mixed regime (producing columnar films), and (iii) particle dominant regime (termed as FAD producing granular films) [321][428], as seen on the left side of Fig. 11. Classification of the deposition mechanism is dependent on the competition of various characteristic time scales in the flame synthesis process, such as residence time in the reactor $\left(\tau_{\text {res }}\right)$, chemical reaction time of the precursor $\left(\tau_{\text {chem }}\right)$, sintering time $\left(\tau_{\text {sint }}\right)$, and collision time $\left(\tau_{\text {coll }}\right)[428]$. In the vapor dominated (CVD) regime, the residence time is significantly lower than the precursor chemical reaction time $\left(\tau_{\text {res }}<<\tau_{\text {chem }}\right)$; thus the vapor only reacts on the substrate, resulting in the formation of a dense film. In the particle dominated (FAD) regime, the residence time is much longer than the chemical reaction time $\left(\tau_{r e s}>\tau_{c h e m}\right)$; and the particles form before deposition on the substrate, resulting in highly-porous granular films. The particle dominated regime can be further sub-divided into two pathways based on the competition between the collision time and sintering time of particles, i.e. individual particle deposition (IPD) when $\tau_{\text {sint }}<\tau_{\text {coll }}<\tau_{\text {res }}$, and agglomerated particle deposition (APD) when $\tau_{\text {coll }}<\tau_{\text {sint }}<\tau_{\text {res }}$ [321]. Finally, if the residence and reaction times are competitive with similar magnitude, then a mixed particle-vapor mixture deposits on the substrate, resulting in the growth of columnar structured films. As for their applications for solar energy conversion, in contrast to the dense 
and columnar films, the porous granular film ( $>95 \%$ porosity) greatly exceeds a requirement of about $40 \%$ porosity for efficient electron transport in films. Because of these differences in electron transport and lifetime in the $\mathrm{TiO}_{2}$ film, the columnar morphology outperformed the granular morphology, achieving a UV-light to hydrogen conversion efficiency of $11 \%$ for water splitting and a visible light to electricity conversion efficiency of $6.0 \%$ for the dye-sensitized solar cell [322].

A controlling diagram portraying the three regimes, i.e. chemical vapor deposition, aerosol deposition, and the mixed one, can be plotted with the particle diameter as the abscissa and the size distribution function of particle number concentration as the ordinate, as shown in the right half of Fig. 11. Here, in order to avoid the complexity induced by the flame, a cleaner system of diffusion-dominated aerosol chemical deposition process (ACVD) was specially designed to study this deposition diagram [428], in which the heat is provided from the substrate itself, instead of flame, depressing thermophoretic deposition, which will be detailed later in Section 5.3. By using a population balance model (as seen in Section 7) implemented in a stagnation-point flow geometry (with nozzle gas mean velocity of $0.2-0.5 \mathrm{~m} / \mathrm{s}$, and correspondingly strain rate of $20-50 \mathrm{~s}^{-1}$, under a nozzle-to-substrate distance of $10 \mathrm{~mm}$ ), the experimental data of ACVD are theoretically interpreted as three kinds of curves (blue, red and black) as shown in the right of Fig. 11. The zone above the blue color line is the aerosol deposition (AD) regime for producing porous granular films (indeed compared with diffusion-dominated $\mathrm{AD}$, the thermophoresis-favored $\mathrm{FAD}$ more easily results in the granular regime under the same particle diameters and concentrations, as deduced from Section 5.3), while the zone below the black color line is CVD regime for producing dense films. The zone encompassed by red color lines is the vapor-particle mixed regime for creating columnar films, while the other zones are 
intermediate or unclear ones to be explored. Basically, the crystalline sizes measured by XRD $\left(d_{X R D}\right)$ or specific surface area (SSA) sizes of the nanoparticles in the FSP-deposited films are about 10-20 nm; and usually those made by PSF-deposition are much smaller at 5-10 $\mathrm{nm}$. Nevertheless, they are all larger than $1.6 \mathrm{~nm}$ in the plot of Fig. 11, evincing an FAD regime for porous granular film.

So far, the well-demonstrated flame deposition processes for the fabrication of thin films mainly include: (i) the first category for CVD technique, i.e., combustion chemical vapor deposition (CCVD) for oxide dense films (firstly proposed by Hunt, Carter and Cochran [415][416] at Georgia Tech and subsequently developed by Liu et al. [417]), along with the CCVD-like technique termed flame-assisted vapor deposition (FAVD) by Choy and co-workers [418] at the Imperial College for synthesis of LSM $\left.\left(\mathrm{La}_{1-\mathrm{x}} \mathrm{Sr}_{\mathrm{x}} \mathrm{MnO}_{3-\delta}\right) / \mathrm{YSZ}\right)$; (ii) the second category for FAD technique, i.e., VAFS-based process detailed in Section 5.5 and FSP-based process in Section 5.6, respectively; and (iii) the combined flame-assisted aerosol-chemical vapor deposition process [419]. In contrast to CVD using gas species as the depositing carrier, the sizes of particles before their deposition in the FAD process is of great significance. Basically, the crystalline sizes measured by XRD $\left(d_{X R D}\right)$ or specific surface area (SSA) sizes of the nanoparticles in the FSP-deposited films are about 10-20 nm; and usually those made by FSP-based VAFS deposition are much smaller at 3-10 nm due to the high quenching rates. Remarkably, between these two kinds of VAFS- and FSP-deposition techniques, Maric and co-workers [420]-[424] have developed a scalable novel flame deposition process, also known as reactive spray deposition technology (RSDT), which can achieve extra narrow particle-size distributions of $0.5-2 \mathrm{~nm}$ for pre-deposited nanoparticles. Nevertheless, the sizes of particles in most FAD processes are usually larger than $1.6 \mathrm{~nm}$, as seen in the plot of Fig. 11, evincing an FAD regime for porous granular film. The 
larger the particles are, the more porous the film is.

\subsection{Key parameters controlling flame aerosol deposition}

In the FAD regime, when $\tau_{\text {res }}>\tau_{\text {chem }}$, the morphology of granular films is directly determined by the deposition/sintering process on the substrate after the formation of particles in the flow field. Based on the premixed stagnation flame burner, as shown in Fig. 2 (f.4), Li and co-workers systematically examined the roles of two important operating parameters, i.e. precursor concentration and substrate temperature, in controlling SSA and porosity of nano-films [10]. As shown in Fig. 12, two distinct regimes were clarified at different substrate temperatures. At low temperatures $(\mathrm{T}=383 \mathrm{~K})$, particles are directly frozen after deposition on the cold substrate, as the on-substrate sintering is quite low. The SSA of the produced films mainly depends on the collision-coalescence process in the gas-phase flame, which is thus termed as the in-flame-agglomeration regime. In this regime, as the precursor concentration increases from $29.1 \mathrm{ppm}$ to $116.4 \mathrm{ppm}$, SSA dramatically decreases from 313.2 to 188.3

$\mathrm{m}^{2} / \mathrm{g}$, because high as-synthesized particle concentrations directly determine the large particle sizes and SSA of the films.

In contrast, at high substrate temperatures $(763 \mathrm{~K})$, the SSA of film is not controlled by the collision-coalescence process of particles in the gas-phase, but by the sintering process upon particle deposition on the substrate. Because the characteristic sintering time of solid-state particles is proportional to the particle volume, the sintering rate reduces rapidly for larger particles. Therefore, on-substrate sintering slows down exponentially with sintering degree of the film, and the particle size has an upper limit after sufficiently long sintering duration. As shown in Fig. 12, the SSA of synthesized 
films is narrow at a small range of $91.4-129.7 \mathrm{~m}^{2} / \mathrm{g}$, with increasing precursor concentration from 58.2 to $116.4 \mathrm{ppm}$. Since particles have been sufficiently sintered on the substrate, this regime is consequently termed as "on-substrate-sintering"-controlled one.

A criterion to distinguish the above two regimes is the location of particle growth. In flame aerosol deposition using the stagnation flow configuration, particle sizes before deposition are mainly determined by precursor concentrations under a designed flame field, whereas particle sizes after deposition are mainly controlled by substrate temperature, as summarized in the literature by Li and co-workers [10]. However, in other flame synthesis cases, large particle sizes, which may arise from higher concentrations, higher flame temperatures, longer residence time, or changing from VAPS to FSP, can still reduce the sintering rates on the substrate even at high surface temperatures. In these cases, the "on-substrate-sintering" regime will revert back to "in-flame-agglomeration" one.

As for the porosity of synthesized films, on the other hand, the key controlling factors are the direct motion of particles towards the substrate caused by thermophoresis (in most cases) and the Brownian diffusion of particles induced by the impaction of gas molecules. The direct motion of particles will lead to the particles penetrating towards inner depths of the films until the Brownian stochastic motion causes their collision with prior-deposited agglomerates. Thus, small Brownian motion and large thermophoresis of particles will cause a large penetration depth of particles, resulting in a high packing density. Additionally, high precursor concentration will lead to a large particle size with small Brownian motion and thus more densely-packed films. The effect of substrate temperature on packing density is relatively weak as it has counteractive influence on thermophoresis, Brownian motion, and on-substrate sintering. In general, high substrate temperatures $(>700 \mathrm{~K})$ will lead to high packing density of films, as 
sintering will dominate.

Another important factor in controlling film porosity is the agglomeration process. Mädler et al. [223] studied the effect of agglomerate morphology, including the size and number of primary particles and fractal number on deposition in a simulation study by solving the Langevin equation. Film porosity increases with increasing the number of primary particles and decreases with higher fractal number. Film porosity only increases with primary particle size when diffusion dominates the deposition process because the deposition velocity will not change with primary particle size when thermophoresis dominates. Based on their simulation and analysis, the agglomerates behave with similar transport physics, but provide larger areas for interception, forming more porous films.

\subsection{Characterization of particle deposition in boundary layers}

Apart from film penetration and sintering on the substrate, another important stage is the transport of nanoparticles within the boundary layer. To further detail the deposition mechanism during this stage, various models of particle transport and deposition within boundary layers have been established. Batchelor and Shen studied the deposition mechanisms of microparticles by considering the competition between thermophoresis and convection (while ignoring Brownian motion) [429]. Mädler et al. estimated the contribution of thermophoresis, Brownian diffusion, and convection, and directly established a thermophoresis-controlled deposition model [222]. More recently, Li and co-workers developed a strictly-derived mathematical model of nanoparticle deposition consisting of convectiondominated, transition, and thermophoresis-dominated regimes, successively, from the outside flame ambience to the inner thermal boundary layer [323], as schematically shown in Fig. 13 (a). 
The transport and deposition of particles via convection, thermophoresis, and diffusion can be expressed as

$$
\nabla \cdot\left(\vec{U}_{c}\right)+\nabla \cdot\left(\vec{v}_{t h} c\right)-\nabla \cdot(D \nabla c)=0
$$

where $U$ is the velocity field at the boundary layer; $c$ is the number density of particles; $v_{t h}$ is the thermophoretic velocity of particles; and $D$ is the diffusion coefficient of particles. With the boundary layer assumption, Eqn. (5.1) is simplified to

$$
w \frac{\partial c}{\partial z}+\frac{\partial v_{t h} c}{\partial z}-w c \frac{1}{\rho} \frac{\partial \rho}{\partial z}-\frac{\partial}{\partial z}\left(D \frac{\partial c}{\partial z}\right)=0
$$

Fig. 13 (b) plots the profiles of thermophoretic velocity, axial gas velocity, and temperature versus axial position. In the convection-dominated regime, thermophoretic velocity is much smaller than the axial velocity, and particle diffusion can also be ignored due to the large Sc number $\left(\sim 10^{2}\right)$. Therefore, the simplified relationship in the convection-dominated regime can be expressed as

$$
\begin{gathered}
\frac{1}{c} \frac{\partial c}{\partial z}=\frac{1}{\rho} \frac{\partial \rho}{\partial z} \\
\frac{c}{c_{0}}=\frac{\rho}{\rho_{0}},
\end{gathered}
$$

where $c_{0}$ and $\rho_{0}$ are the number density of particles and the density of gas, the upstream of the boundary layer, respectively. This relationship indicates that the ratio of the particle number to the gas density is constant, and the change of particle number density is mainly attributed to the higher density gas (e.g. by 
way of ideal gas) near the cold boundary layer.

In the thermophoresis-dominated regime within the inner stationary layer, convection is negligible compared with thermophoretic velocity. Defining non-dimensional variables, $C=c / c_{1}$ and $Z=z / \delta_{s t}$, where $c_{1}$ is the number density of particles at the outside of stationary layers and $\delta_{s t}$ is the thickness of the stationary layer, Eqn. (5.2) can be simplified as:

$$
P e_{t h} \frac{\partial C}{\partial Z}-\frac{\partial^{2} C}{\partial Z^{2}}=0
$$

The change of $v_{t h}$ is generally within $10 \%$ under the studied cases, and $\delta_{s t}$ is very small such that variations of $\mathrm{D}$ can be ignored. Here, $P e_{t h}=v_{t h} \delta_{s t} / D$ is defined as the thermophoretic Peclet number describing the ratio of thermophoresis and diffusion flux. With the boundary conditions of $\left.C\right|_{Z=0}=0$, $\left.C\right|_{Z=1}=1$, Eqn. 5.1 can be solved analytically; and the solutions of the number density of particles and the deposition flux are:

$$
\begin{aligned}
C & =\frac{1-e^{P e_{t h} Z}}{1-e^{P e_{t h}}} \\
\frac{\phi}{c_{1} v_{t h}} & =\frac{e^{P e_{t h}}}{e^{P e_{t h}}-1}
\end{aligned}
$$

When $P e_{t h}$ is larger than 1, i.e. thermophoresis is greater than diffusion, the right-hand side of Eqn. (5.6) approaches 1; and the deposition flux is equal to the thermophoretic flux of particles. As shown in Fig. 14, when the particle size is larger than $4 \mathrm{~nm}$, the thermophoretic Peclet number is much larger than 1 , and the Brownian diffusion of particles contributes little to the deposition of particles. 
As for the entire boundary layer, the particle concentration can be solved directly from Eqn. (5.2). The particle concentration will first increase slightly due to the higher gas density in the convection-dominated regime. At the thermophoresis-dominated regime, the particle concentration does not change significantly as indicated by Eqn. (5.5), when $P e_{t h}$ is much greater than 1 . Finally, the particle concentration drops rapidly after entering the concentration boundary layer, which is determined by diffusion and thermophoresis together. In general, the concentration boundary layer is much thinner than the stationary layer, and the variation of particle diameter will not influence the deposition flux. However, small particle size $(<2 \mathrm{~nm})$ will lead to a comparable diffusion flux with thermophoresis, increasing the concentration boundary layer thickness equivalent to or even thicker than the stationary layer. In this case, the peak particle concentration will be reduced, causing a reduction in deposition flux.

\subsection{Control of deposition by electric field}

The use of force fields during the formation and deposition of nanoparticles offers another potential tool for process control. The application of electric fields stands as an advantageous means for precise control of primary particle size, crystallinity, aggregate size [1], and deposition characteristics. The effects of electric fields on flames have been observed and reported in the literature for a long time [430][431][432][433]. Past investigations of electric field application in traditional hydrocarbon

combustion systems have demonstrated control of various flame characteristics (e.g. burning velocity [434]-[436], stability [437][438], shape [439]-[441], luminosity [442][443], extinction limit [444]-[446]) and pollutant formation (e.g. soot [439][441][443][447], NOx [444][448]). In absence of particles, the 
effects of an electric field on flames are now attributed to ion-driven fluid-mechanical effects, known as ionic (or Chattock [449]) winds effect. Under an electric field, ions are accelerated preferentially toward an electrode. At the same time, they collide with neutral molecules, transferring a portion of their momentum to the neutral molecules. The net result is that the electric field produces a significant body force or "ionic wind". Owing to such aerodynamics, the entire flow field can be affected, thereby influencing flame characteristics.

Subjecting an external electric field to the particles during their formation and deposition has been found to have a significant effect on primary particle size, crystallinity, degree of aggregation, and agglomerate size. Early on, Hardesty and Weinberg [450] showed that the primary particle size of $\mathrm{SiO}_{2}$ powders was reduced by a factor of three for a $6 \mathrm{kV}$ potential in a Meker-burner-supported, premixed methane/air flame. Katz and Hung [244][451] found an opposite trend in particle size in a counter-flow diffusion $\mathrm{H}_{2} / \mathrm{O}_{2}$ flame when an electric field was applied across it. Regardless of the polarity of the bottom electrode, dynamic laser scattering and TEM probing data showed a significant increase in the diameter of the particles $\left(\mathrm{TiO}_{2}, \mathrm{SiO}_{2}\right.$, and $\mathrm{GeO}_{2}$ ) produced (a 3-10 fold increase in some cases) compared with those produced without an electric field.

Much of the work in electrically-assisted flame synthesis of particles has been done by Pratsinis and coworkers [452]-[455]. They have investigated flames in various geometries, generally with electric fields applied perpendicular to the direction of flow using needle-shaped or plate electrodes. In Ref. [452], they produced ultrafine titania particles generated from a jet $\mathrm{CH}_{4} / \mathrm{air} / \mathrm{Ar}$ diffusion flame using a corona discharge to charge the particles (for repulsion and dispersion) and to reduce residence time through ionic wind effects by flow mixing. Increased electric potential reduced the particle size and the 
rutile content. In Ref. [453], they produced $\mathrm{TiO}_{2}, \mathrm{SiO}_{2}$, and $\mathrm{SnO}_{2}$ nanopowders generated by burner-stabilized flat flames and nonstabilized laminar (Bunsen-like) flames. The fields (applied perpendicular to the direction of flow) were created either by needle electrodes to introduce ions and wind in the flame or by plate electrodes to merely attract flame-generated ions. The electric fields reduced the primary particle size of $\mathrm{TiO}_{2}$, the agglomerate size of $\mathrm{SnO}_{2}$, and both the agglomerate and primary size of $\mathrm{SiO}_{2}$. In Ref. [455], they were able to reduce primary particle diameter (by a factor of two) of fumed silica produced at high rates (up to $87 \mathrm{~g} / \mathrm{h}$ ) using a co-flow double diffusion flame with needle electrodes.

Generally, external electric fields can affect flame synthesis in two main aspects: (i) electric fields can change the flame field and therefore change the histories of the nanoparticles; (ii) electric fields can directly influence synthesized nanoparticles with electrophoretic force $F_{E}$ that can affect the migration/drift velocity $v_{E}\left(\equiv F_{E} / f\right.$, where $f$ is friction coefficient) in the left side of Eqn. (5.1), or with electrostatic dispersion that can change the coagulation rate between charged nanoparticles in the electric field.

The first aspect involves the electric aspect of the flame, which has been reviewed in Refs. [432][456]. Despite many debates, it is generally believed that 'ionic wind', i.e. electro-hydrodynamic effect, is predominantly responsible for flame changes upon weak, sub-breakdown electric fields, as reported by Pratsinis and co-workers. Similar results have also been observed by Katzer et al. [457]. It should be noted that ionic winds, as a weak discharge, can only affect convection in flames. Stronger discharge in plasma-assisted combustion, such as nanosecond discharge, dielectric barrier discharge, microwave discharge, and gliding arc can change flame structure by modifying the chemical reaction 
route apart from the electro-hydrodynamic route, which presents the possibility of controlling nanoparticle characteristics by adjusting the flame structure.

To remove the effect of ionic wind on flame and to isolate the electrophoretic effect of electric field on the transport behaviors of nanoparticles, Tse and co-workers [319] utilized a burner-stabilized premixed stagnation flame with a uniform electric field applied between the burner and the water-cooled substrate. By this geometry, gas-phase temperature and chemical species profiles are unaffected and only transport of the innately charged nanoparticles (by thermionic emission) is induced. The electrophoretic transport of particles can be expressed as $v_{E^{*}} \mathrm{c}$ in Eqn. (5.1), with signs corresponding to the direction of the applied electric field, where $v_{E}$ is the electro-migration velocity. The experimental results showed that the electrophoretic effect can significantly increase/decrease the residence time in the high temperature region of the flame and therefore change the agglomeration process. Both agglomerate and primary particle sizes decrease with concurrent electric field (the substrate with negative voltages and the burner with grounded potential) because of the shorter residence time of nanoparticles. Moreover, electric fields can also change the coagulation rate by charging nanoparticles because of electrostatic dispersion of nanoparticles before their deposition on the substrate, as demonstrated by Vermury et al. [453] and Katzer et al. [457] in the experiments with needle electrode configurations. However, the electrostatic dispersion effect is difficult to separate from ionic wind effects and needs further investigations.

Apart from metal-oxide nanoparticles, electric fields have also been utilized in controlling the packing structure of carbon nanomaterials during flame synthesis. Kennedy and co-workers [458] studied the alignment, growth rate and structural variations of carbon nanotubes (CNT) induced by 
electric fields. A conductive metal-based catalytic probe, connected to an external voltage ranging from $0.3-25 \mathrm{~V}$, was positioned at the fuel side of a non-premixed counter-flow flame. At low voltages below $1 \mathrm{~V}$, the CNT array thickness increase linearly with the applied voltages, while further increase of the voltage begins to modify nanotube morphology. Different structures have been observed with increase of voltages, from high purity vertically aligned carbon nanotube (VACNT) in the range of $0.3-2 \mathrm{~V}$, helically coiled CNTs at $\sim 3 \mathrm{~V}$, and CNTs with multi-channels to multi-junctions at higher voltages. They argued that the strong electric field at the tips of synthesized carbon nanotubes induce a tensile force to generate the helically coiled structures and help to form new catalytic sites for nanotube branching.

$\mathrm{Xu}$ et al. [459] similarly investigated the effect of voltage bias on the metal probe/substrate on CNT growth in an inverse co-flow diffusion flame. The results showed that voltage bias on the substrate was conducive to aligning CNTs, as well as enhancing their growth rates as compared to grounded conditions. Both negative and positive voltages improved CNT alignment and growth (with negative voltages working a bit better). They noted that operating the probe/substrate in floating potential mode resulted in negative voltages (i.e. $24 \mathrm{mV}$, as induced by flame-generated ions and electrons), along with well-aligned CNTs. This was corroborated with experiments done using a counterflow diffusion flame [460].

Future application of electric fields between flame nozzles and substrate should be directed at synthesizing functional nanocomposite thin films, given such limited researches at the moment.

\subsection{VAFS-based fabrication of thin films}

In vapor-fed flame aerosol flame deposition, chemical reaction is very fast, and particles are formed 
prior to their final deposition on the substrate. Particularly in the premixed stagnation flame configuration, thin films are fabricated composed of primary ultrafine particles (around $10 \mathrm{~nm}$ or less) with large specific surface area (SSA), resulting in highly porous films. However, high porosity also means low mechanical stability. Thus, direct VAFS-grown films may not be a good choice for coating materials in a flow environment. In addition, the electron transport in highly porous granular films is largely restricted due to the discontinuity of the neighboring particles, which results in poor performance in the application of flame-made films for gas sensors and solar cells. In recent research on VAFS-based fabrication of thin films, post-treatment methods, including film densification and sintering, are becoming more and more important to meet the various application demands.

As shown in Fig. 15 (A), the flame stabilized on a rotating substrate (FSRS) method (variation of the premixed stagnation flame burner), has been demonstrated to be a successful operational configuration for VAFS-based fabrication of thin films [311][318][461][462]. Developed by Wang and co-workers, the VAFS-fabricated method is able to produce inexpensive films for gas sensors and solar cells, as shown in Fig. 15 (B-C). The flame in the setup is a premixed flat flame generated from an aerodynamic nozzle, stabilized in a laminar stagnating flow against a rotating plate, which is similar to the stagnation flames introduced in Fig. 2 (f3). The plate here is convectively cooled so the particles are driven by thermophoresis to form the thin film. In Fig. 15 (D), $\mathrm{TiO}_{2}$ films are grown on the substrate by the FSRS method [461]. The thickness of the film reaches $14 \mathrm{um}$ in five minutes, showing a high fabrication rate which is of value to both research and manufacturing. The nascent film has a high porosity, i.e. $>90 \%$; and the mean primary particle diameter composing the film is 8-9 nm.

The densification process in FSRS-fabricated thin films is notable as the porosity is effectively 
decreased to a desirable value, i.e. $\sim 50 \%$ after post-treatment. In the densification method, the as-synthesized film is densified by wetting it with a solution of ethyl cellulose $(15 \mathrm{wt} \%)$ in ethanol. Then the wet film is heated stepwise to evaporate the ethanol and to stabilize a much denser structure. The morphology change of the film after densification can also be seen in Fig. 15 (D). After such densification, only the porosity of the film is changed; other properties like mean particle size are still maintained. Apart from adding ethyl cellulose, high-temperature annealing/calcination is also a common densification method [463]. The annealing process can decrease the porosity and lower the concentration of defects. Nevertheless, the process will also cause some grain growth, which may change the particle size distribution of the film.

Fig. 15 (B) and (C) are two different application tests on thin films made through VAFS fabrication and densification. The performance of gas sensing for $\mathrm{CO}$ is shown in Fig. 15 (B). The baseline of the resistance profile is quite stable, and no significant drift happens over time, demonstrating the stability and sensitivity toward CO sensing [461]. The thin film is still sensitive under 5 ppm $\mathrm{CO}$ exposure. In Fig. 15 (C) the film is tested for photo-electric performance. The photo-conversion efficiency reaches as high as $8.2 \%$, with a short-circuit current density of $20 \mathrm{~mA} / \mathrm{cm}^{2}$, which is higher than those of typical champion solar cells [462]. These desired features of the film are attributed to the smaller particle size obtained by VAFS which provides good sensitivity and activity in application, as well as good mechanical and chemical stability of the film after densification.

\subsection{FSP-based fabrication of functional film and devices}

Different from VAFS-based fabrication utilizing the premixed stagnation flame, more functional 
films and devices can be made using FSP, given its broader range of available precursors for synthesis and doping. For example, a large variety of semiconducting metal-oxide materials have been synthesized via FSP, which have been partially included by Kemmler et al. in Table 1 in a previous review [219]. In brief, $\mathrm{SnO}_{2}$, an n-type semiconductor, perhaps the most successful semiconductor oxide, has been investigated in detecting $\mathrm{CO}$ [222] and $\mathrm{EtOH}[82] . \mathrm{WO}_{3}$, known to be non-stoichiometric with two different stable crystalline phases [465][475], shows great potential in $\mathrm{NO}_{2}$ gas detection [475]. ZnO is chemically and thermally stable, and has high sensitivity to toxic and combustible gases [132][466][467][468]. $\gamma-\mathrm{Fe}_{2} \mathrm{O}_{3}$ attracts research interest because of its high sensitivity, low cost, quick response, and low power consumption [469]. $\mathrm{Co}_{3} \mathrm{O}_{4}$ [470][471], $\mathrm{CuO}$ [472][473], and other p-type semiconducting metal oxides indicate great potential in detecting gases at low temperatures with low response and recovery time. Moreover, surface sensitizers and bulk dopants can be easily added in one-step in the FSP process, which is one of the most successful strategies to improve the performance of functional films and devices. It has been reported that Pt [219], Pd [171] and $\mathrm{Cu}$ [109] are used for surface sensitizers, while $\mathrm{Sb}$ is used to tune the resistivity of $\mathrm{SnO}_{2}$ as bulk dopants [215] and $\mathrm{Sn}$ for $\mathrm{In}_{2} \mathrm{O}_{3}$ [216]. As a representative work, consider Pt-doped $\mathrm{SnO}_{2}$ [219], where the higher the loading of a material with a noble metal, the higher the conversion of dopants to analytes, instead of delivering of electrons to the conduction band of the semiconducting metal oxide. Therefore, an optimum concentration exists for the incorporation of additives in the precursors. The second important feature of FSP-fabricated functional films is the relatively simple post-fabrication procedure, as compared to the complex processes associated with liquid-based methods. In a typical FSP-fabricated process, particles are directly deposited onto a water-cooled substrate, ensuring a large thermophoretic deposition rate. A 
mask is usually used to deposit particles within the desired sensor area. Subsequently, the sensor substrate with deposited particles is then tested in a DC electric circuit to assess its sensitivity $\alpha$, lower detection limit LDL, and reproducibility $Q$, as defined by Mädler et al., under certain gaseous environments [222].

However, due to the high-porosity, the deposited films may not be mechanically stable, significantly limiting the use of FSP-fabricated films in liquid environments. To solve this problem, Kühne et al. and Tricoli et al. combined FSP with recent advances in microtechnology and developed a new generation of micromachined, smart, single-chip gas sensors, based on the direct deposition of FSP on wafer-level to minimize power consumption [224][464]. Figure 16 (a) shows the details of the fabrication processes, as previously reviewed in literature [9]. An FSP unit was combined with a water-cooled substrate for synthesis, and a shadow mask was used in the direct deposition of thick layers of nanoparticles. The nanoparticles also deposited through the pores of the shadow mask directly onto micro-hotplates, which were micro-machined on wafers with a sensitive area of $300 \times 300 \mu \mathrm{m}^{2}$, Pt-interdigitated electrodes, an embedded heater, and three temperature sensors. During deposition, the substrate was maintained around $150{ }^{\circ} \mathrm{C}$ to ensure a large thermphoretic deposition rate. Then, the layers were annealed in situ by impinging a particle-free xylene spray flame onto the shadow mask for 30-60 s to achieve mechanically stable layers. Further details on the fabrication have been presented in Ref. [224]. It should be pointed out that the particle deposition at the first step is characterized by a clear "in-flame-agglomeration"-controlled regime because of the low substrate temperature, while the subsequent in situ annealing is distinguished by an "on-substrate-sintering" regime at high flame temperature, where lace-like layers change to compact cauliflower-like structures with a substantial 
reduction of SSA. The separation of the two regimes at different stages of FSP-fabrication produces novel micro-patterned stable functional inorganic nanomaterial layers.

A different stabilization method is proposed by Mädler and co-workers by mechanically laminating the FSP-fabricated highly porous filter cake into a final dense film at room temperature, leading to mechanical restructuring and stabilization of the porous layers [474]. An analysis of pore size indicates homogenization of the layers through rearrangement of the aggregates inside the layers, increasing with applied pressure.

Apart from the thermophoresis-dominated deposition mechanism, an interesting direct impaction deposition mechanism has been proposed and developed. To control the lateral resolution of the coatings and to achieve mild processing, low contamination, and compatibility with microfabrication processes, Wegner et al. employed a supersonic cluster beam deposition system in-line with an FSP reactor [476]. Fig. 16 (b) schematically shows the details of the FlameBeam apparatus which consists of an FSP reactor, a critical flow quenching nozzle, an aerodynamic lens focuser, and a sample holder for substrate raster, all confined within a vacuum deposition chamber. The FSP-synthesized nanoparticles go through the quenching nozzle and are focused by aerodynamic lenses into a beam of $\sim 25 \mathrm{~mm}$ diameter onto the substrate. Particles that pass through the lenses are small with large Stokes number. Therefore, the traditional thermophoresis-dominated deposition regime in Section 4.3 shifts into a directed impaction process, similar to capabilities of high-velocity oxy-fuel (HVOF) processing [477]. The substrate temperature control system is not needed and coatings can be deposited on temperature-sensitive materials such as polymers. Moreover, the final deposited nanoparticles are isolated from volatile species such as water or unburned precursors that could contaminate the product film. 
Another alternative to abating the thermophoresis-dominated deposition is to precisely control the particle size before deposition. As mentioned, Maric and co-workers [420]-[424] have developed an FSP-based technique, RSDT. Different from traditional FSP deposition processing, precursors can be heated in a reactor vessel with temperatures up to $150^{\circ} \mathrm{C}$ and pumped with pressures up to 69 bar, which permits a supercritical solution into the atomizer [420]. This RSDT ensures a fast solvent vaporization process followed by a direct gas-to-particle conversion route (instead of droplet-to-particle route), as shown in Fig. 1. This bottom-up approach helps to realize small nanoparticle sizes before deposition (e.g. down to 0.5-2 $\mathrm{nm}$ of Pt particles [424] ). This small nanoparticle size (around 1-2 $\mathrm{nm}$ ) leads to a comparable diffusion flux with respect to thermophoresis, resulting in much denser films than those achieved by traditional FSP-based deposition. Indeed, this kind of novel processing with generating ultra-fine droplets, typically as an improved FSP technique with its scalability and material adaptability, can also have great potential when used in the premixed stagnation flame (PSF) system. As a variation of VAFS-based fabrication (Section 5.5), it can now utilize a variety of low volatility metal precursors and still control their particle growth at atomic precision.

Moreover, the FSP-fabrication technique is easily scalable. For example, the capability to operate continuously at atmospheric conditions makes FSP an interesting technique to control the wettability of surfaces (i.e. paper and board). FSP-synthesized $\mathrm{SiO}_{2}$ film coatings have been reported to provide excellent hydrophilicity, where the film coating on paper can achieve high production line speeds up to $150 \mathrm{~m} / \mathrm{min}$, with great potential for industrial utilization [478].

\section{Diagnostics for nanoparticle synthesis}


In the last 20 years, significant advances in diagnostics of combustion aerosol formation have permitted a significant improvement in understanding physical processes, providing data for testing models, and remote monitoring and robust controlling in high-temperature multiphase reaction environments. Here, we summarize the diagnostic methods in two aspects: (i) intrusive diagnostics based on sampling, and (ii) on-line in situ diagnostics based on optical and laser techniques.

\subsection{Intrusive diagnostic based on sampling methods}

Sampling diagnostics provide detailed information of synthesized nanomaterials, such as morphology and size (by SEM/TEM), size distribution (by SMPS/DMA), crystallinity (by XRD), surface morphology (by SEM), and average size (by BET). Compared with full-scale materials characterization technologies, sampling and collection of nanoparticles from high-temperature aerosol environments are relatively difficult, as minimizing disturbances requires understanding of particle dynamics and their interactions with fluids. Various in situ and ex situ diagnostic methods in flame synthesis are summarized in Fig. 17.

Thermophoretic sampling with subsequent TEM analysis (TS-TEM) is regarded as the most straightforward method, with little disturbance on the fluid flow field. This method can divulge the relatively real morphology of as-synthesized particles because the $\sim 10^{1}-10^{2} \mathrm{~ms}$ residence time of the cold TEM grid in the flame field ensures the lack of coagulation during the sampling process. Moreover, the thermophoretic velocity of nanoparticles is independent of particle diameter in the free molecular regime. Primary nanoparticles of $\mathrm{TiO}_{2}$ [96][304][479][480], $\mathrm{SiO}_{2}$ [304][480], and $\mathrm{Al}_{2} \mathrm{O}_{3}$ [308] have been sampled during flame synthesis; and the statistical averaged particle size is usually treated as 
accurate for studying novel laser diagnostics and examining modeling results [296]. Sizes of agglomerates or aggregates have also been diagnosed by TS-TEM [304][308][481]. Gröhn et al. [481][482] compared the sizes of agglomerates measured by TS-TEM and DMA-APM, as exhibited in Fig. 18. Consistent measurements of primary particles are obtained by TS-TEM and DMA/APM. However, as soon as aggregation begins, gyration diameters of agglomerates become larger than projected area equivalents and mobility diameters. As shown in the inserted plot of Fig. 18, when agglomerates grow from the free-molecular regime to the continuum region, mobility diameters gradually exceed projected area equivalent diameters, which gives the upper limit of this sampling method. In general, TS-TEM is an off-line diagnostic method and can only reflect the integrated result along the sweep route of the TEM grids. Nonetheless, it interferes the least with an aerosol process due to small probe size, flow orientation and short residence time and ensures high-certainty result.

For more detailed information like size-distribution, aggregate number, average primary particle diameter etc., a well-designed sampling system is required to extract particles from the high-temperature flame field and to keep the original morphology of the nanoparticles. To avoid particle coagulation in the sampling tubes and diffusion loss to their boundaries, as-synthesized nanoparticles are diluted significantly during sampling to ensure that the flow time is much smaller than the collision time of the particles as well as the diffusion time to the boundary of the sampling tube. Several typical dilution systems are shown in Fig. 17 (a-c). Wang and co-workers [483] established a dilution sampling system for measuring the size distribution of $\mathrm{TiO}_{2}$ nanoparticles by a scanning mobility particle sizer (SMPS) in which a large flow rate about $29.5 \mathrm{~L} / \mathrm{min}$ ensures a large dilution ratio about $10^{5 \sim 7}$, as exhibited in Fig. 17 (a). Tse and co-workers [320] extended the measurements to low-pressure by developing a sampling 
system with an intermediate aerosol chamber (based on the works of Ober et al. [484] and Whitby and Hoshino [485]), with three stages of dilution, coupled to commercial nano-SMPS instrument operating at atmospheric pressure. To enable proper characterization of the nanoparticles, the measured PSDs were compared with computations from a sectional model, which considered continued particle growth within the sampling line and the aerosol chamber. Different sampling probe methods were utilized in studies of Pratsinis and co-workers [490], Biswas and co-workers [486] and Roth and co-workers [280][489], as depicted in Fig. 17 (b), (c), (g), respectively. Biswas and co-workers first extended the measurement of particle size distribution to the sub-2nm region by a half mini differential mobility analyzer (Half Mini DMA) and a Faraday cup electrometer (FCE) [486][487][488]. Particle mass spectrometry (PMS) was also used as an in situ technique to follow the evolution of particle size. Roth and co-workers first used a molecular beam technique and an aerosol particle mass analyzer (APM) to probe particles in a low pressure flame [280][489], as depicted in Fig. 17 (g). First, charged particles are led to a $10^{-6}$ mbar volume by a nozzle and skimmer, are then classified by an electrical deflection and filter system based on their kinetic energy and velocity, and are finally detected by a FCE. Pratsinis and co-workers added a DMA before an APM and obtained quantitative information about particle morphology without assumptions of agglomerate size distribution [490]. In general, intrusive diagnostic methods can provide in-situ high-resolution data based on the powerful aerosol anlysis instruments, but the measurement uncertainty is usually limited by the sampling process because (i) the sampling probe may disturb the local high-temperature aerosol flow field; (ii) the collection efficiency of the sampling system needs to be calibrated with high accuracy; (iii) the size distribution of nanoaerosol should be freezed during the sampling process. 
For ex situ measurements, synthesized samples can be directly obtained from stagnation substrates or captured by filters, as shown in Fig. 17 (e), (d). TEM, SEM, and BET are then used for characterization. Measurements from substrates are detailed in the works of Li, Tse and co-workers [10][319][320][323], Wang and co-workers [316][317], Maric and co-workers [421][422], Mädler et al. [222], and Biswas and co-workers [322], etc. Water-cooled substrates quench the coagulation process of nanoparticles and ensure thermophoresis-dominated deposition, since $P e_{t h}>1$ at the inner boundary layer [10][323]. Nano-devices like sensors or nano-films, can be directly characterized from the deposited materials on the substrates. In industrial applications, synthesized nanoparticles are usually captured by filters, as demonstrated in experimental studies of Pratsinis' group at the laboratory [43] and industrial levels [327].

\subsection{On-line optical measurement}

Compared with intrusive sampling, optical measurement especially laser-based diagnostics provide the capability for robust, non-intrusive, in situ measurements with both spatial and temporal precision. Utilization of optical diagnostics for flame synthesis can be categorized into two main objectives: (i) to provide comprehensive and reliable data of the gas-phase combustion or chemical reactant (including precursor) field, and (ii) to directly diagnose the as-synthesized nanomaterials in situ. These two objectives combined will clarify the different sub-processes during flame synthesis, such as chemical reactions, nucleation, doping, and recrystallization.

\subsubsection{Diagnostic of flame field}

Table 2 lists optical diagnostic methods that can cover various parameters of the flame-synthesis 
flow field.

Spontaneous Raman spectroscopy (SRS) can give a quantitve measurement of flame temperature with an uncertainty of $\pm 50 \mathrm{~K}$. SRS can be divided into rotational or vibrational scattering methods, corresponding to different levels of inelastic scattering between laser light and molecules. Allendorf et al. [491] studied the influence of silica nanoparticles on flame structure by using Raman spectroscopy and suggested $\mathrm{N}_{2}$ as the best choice for Raman measurement. Our co-workers Liu et al. [492] and Zhang et al. [493] utilized $\mathrm{N}_{2}$ Raman to determine gas-phase temperatures. SRS is self-calibrating and relatively easy to operate but weak in intensity and not suitable for highly luminous particle-laden flames due to interference [494].

Coherent anti-Stokes Raman spectroscopy (CARS) was developed as an approach to enhance Raman signal levels, in which the vibrational fingerprints of certain molecules are measured by phase mixing during the intersection of three laser beams. Bertagnolli and Lucht [315] used $\mathrm{H}_{2}$ CARS to measure temperature profiles near the deposition wall in a diamond-forming flame, while Kammler et al. [278] applied $\mathrm{N}_{2}$ CARS under a single pulse laser pulse to measure the temperature profile in a premixed flat flame producing $\mathrm{TiO}_{2}$ nanoparticles. By utilizing $\mathrm{O}_{2}$-based pure rotational CARS for temperature measurement, along with laser induced breakdown detection (LIBD) for droplets and particles, Engel et al. [479] discovered that the energy consumption during particle formation leads to significantly lower gas temperature with higher particle concentration during $\mathrm{SiO}_{2}$ formation from $\mathrm{HMDSO}$ in FSP. However, using $\mathrm{N}_{2}$-based CARS during VAFS of $\mathrm{SiO}_{2}$ from $\mathrm{SiCl}_{4}$, Choi and co-workers discovered that temperatures decrease in the preheat zone due to the increase in specific heat of the gas mixture, while temperature decrease is mitigated in the particle formation zone due to heat release through hydrolysis 
and oxidation reactions of $\mathrm{SiCl}_{4}$ [496][497]. CARS can be widely used in flame synthesis with highly radiant backgrounds. The high precision of temperature measurement of CARS $(<4 \%)$ makes it possible to calibrate other techniques. However, CARS may be complicated in operation, along with requiring sophisticated facilities.

Fourier transform infrared spectroscopy (FTIR) measures temperature from transmission and emission spectra by fitting a blackbody Planck function to normalized radiance of high-temperature spectra [41][498][499] (for which the $\mathrm{CO}_{2}$ peak is most reliable spectra [499]). This approach has been applied to make temperature measurements in flame spray synthesis of $\mathrm{SiO}_{2}$ [41], $\mathrm{ZrO}_{2}$ [328], $\mathrm{Pt} / \mathrm{TiO}_{2}$ [500], $\mathrm{TiO}_{2}$ [278], and $\mathrm{Fe}_{2} \mathrm{O}_{3}$ [501]. It should be noted that FTIR makes flame temperature measurements as an average value along the IR beam; and therefore several FTIR measurements need to be taken and then tomographically reconstructed to map the non-uniform flow [498][503].

Laser induced fluorescence (LIF) possesses the capability to measure radical species concentration and temperature locally by tuning a laser to specific wavelengths for absorption by certain molecules, and then by observing the relatively-intense spontaneous emission (fluorescence) at red-shifted wavelengths (thereby improving SNR) from excited-energy-state molecules. Hwang et al. (Choi's group) [496] employed a PLIF technique to measure relative profiles of $\mathrm{OH}$ concentrations, which decreased significantly in particle formation zones due to silica generating reactions of $\mathrm{SiCl}_{4}$. Glumac et al. [502] employed multi-line OH LIF thermometry to diagnose the temperature and radical fields in a low pressure $\mathrm{H}_{2} / \mathrm{O}_{2}$ flame combined with laser induced scattering which is used for $\mathrm{SiO}_{2}$ synthesis. Tse and co-workers [319] isolated the electrophoretic effect on nanoparticle size and agglomeration (by manipulating particle residence times) in electric-field-enhanced flame synthesis by comparing LIF of 
gas-phase temperature and $\mathrm{OH}$ concentration with computational simulations, showing negligible differences between cases with and without uniform electric field. Kronemayer et al. [504] and Hecht et al. [505] from Schulz's group obtained the temperature field by multi-line NO-LIF thermometry in $\mathrm{TiO}_{2}$ and $\mathrm{Fe}_{2} \mathrm{O}_{3}$ formation in VAFS, respectively.

Another aspect of LIF application is to diagnose intermediates in precursor decomposition/reactions. Different excitation lasers are used for different kinds of intermediate monomers, as summarized in Table 3. The LIF signals of intermediates like $\mathrm{SnO}$ [506], $\mathrm{SiO}$ [507], $\mathrm{FeO}$ [508], Fe [505], $\mathrm{PbO}$ [509], $\mathrm{AlO}$, and $\mathrm{TiO}$ [510] exhibit similar concentration distributions with a sharp rise in number density near the burner and a peak in the profile which moves towards the burner outlet as the precursor concentration increases. Glumac and co-workers [506][507] further concluded that monoxide comes from both the product of precursor decomposition early in the flame and the partial pressure of solid phase in the downstream portion of the flame. Muramoto et al. [511] used a redecomposition LIF (ReD-LIF) to visualize the behavior of Si clusters synthesized in a laser-induced plasma plume, during which the $\mathrm{Si}$ clusters are first decomposed by a $\mathrm{XeCl}$ excimer laser into atoms and then imaged by 2D-LIF. In general, LIF is useful in probing low-concentration species in flames (about several ppm Fe in [505]), but quenching of excited species can restrict its quantitative measurement of species.

Compared with laser-based techniques, optical emission spectroscopy (OES) is qualitative in measuring gas-phase species concentrations, but is more amenable for operation in industrial environments, needing only spectrometers or filter lenses. Apart from the intense emission bands from radicals $\mathrm{OH}^{*}, \mathrm{CH}^{*}$, and $\mathrm{C}_{2} *$ [512], emissions of intermediates in precursor reactions, like $\mathrm{SiO}$ $\left(220-265 \mathrm{~nm}\right.$ ) and $\mathrm{Si}$ (around 252nm) in flame synthesis of $\mathrm{SiO}_{2}$, have also been detected [513]. 
Moreover, OES is a line-of-sight technique which has limitations in spatial resolution.

\subsubsection{Laser diagnostic of particles}

Besides gas-phase diagnosis of the flame synthesis field, non-intrusive in situ laser-based diagnostics of the as-synthesized materials are needed for understanding, modeling, monitoring, and controlling the synthesis process. Table 4 lists various methods of laser diagnoses of particles during flame synthesis. Diagnostics of the particle phase are based on interactions between laser and particle matter, and the interactions at various levels reflect certain information about the particles. Elastic scattering (or laser-induced scattering) of particles can indicate particle size, and inelastic scattering (or Raman) can give information about the crystallinity. For higher laser energies, laser-induced incandescence (LII) can irradiate particles thermally, determining the number density and size of the particles, using certain absorption and radiation models. When the photons of the laser are energetic enough to excite the electrons up to the conduction band of the particles, atomic emission of phase-selective laser-induced breakdown spectroscopy (PS-LIBS) is useful in detecting gas-to-particle conversion and bandgap variation processes of certain compositions, along with determining volume fractions of as-synthesized nanoparticles. Finally, when the laser power is strong enough to breakdown all material to plasma, elements of both gas and particles phases can be quantified by conventional laser induced breakdown spectroscopy (LIBS). We summarize these laser-particle interaction mechanisms in Fig. 22, and discuss them subsequently. 


\subsubsection{Laser and X-ray induced scattering (LIS)}

Laser-induced scattering (LIS) can be divided into two regimes: Rayleigh scattering $\left(d_{p}<<\lambda\right)$ and Mie scattering. Most LIS scattering experiments are conducted in the Rayleigh regime, due to the relatively simple scattering rules compared with the Mie scattering theory for optical light.

In the Rayleigh scattering regime, the scattered light is proportional to the average square of particle volume and the number density of particles. If the system is a pure coagulation, that is, the particle volume fraction (and thus the volume of particle times the number density) is constant, the scattered signal is then approximately proportional to particle volume, which is called the Tyndall effect in the aerosol measurement field [514]. Graham and Horner [515] measured the time-resolved particle diameter in a coagulation system. Yang and Biswas [516] used Rayleigh scattering of particles to evaluate characteristic sintering time. However, most Rayleigh scattering measurements can only be viewed as qualitative because of the invalid Rayleigh-Debye-Gans approximation, which assumes that particles behave like point sources of reradiated waves, with no reflection and internal particle refraction. Moreover, the scattered light is affected due to light extinction through the flame, and therefore additional information by other techniques is necessary for calibration, limiting quantitative LIS. If we expand our consideration into Mie scattering, which can be used to measure the size distribution function of micro-sized droplets or sub-micro particles in FSP, then multi-wavelength light sources [517][518] or multiple scattering angles [308][519] are needed to obtain more data of the aerosols because the scattering kernel is not a simple function of particle size. Ma systematically discussed the mathematical consideration of solving the aerosol size distribution from the Fredholm integral equations 
Small-angle $\mathrm{x}$-ray scattering (SAXS) can successfully overcome the above limitations in scattering of optical light because x-ray frequencies are too high (three magnitudes higher than optical frequency) for most electronic responses which makes the refractive index for x-rays nearly unity. Therefore, without considering wave velocities and boundary conditions, X-ray scattering is simply a wave problem [521]. After solving the technique problem of small fluxes, SAXS is extended from an ex situ diagnostic [522][523] to in situ diagnostic [524][525][276]. Beaucage et al. [524] first applied SAXS using synchrotron radiation in studying $\mathrm{SiO}_{2}$ nanoparticle growth in flames. Camenzind et al. [525] utilized SAXS for monitoring primary particle size, volume fraction, aggregate gyration radius, and mass fractal dimension with good spatial resolutions. Briefly, Porod's law, $I(q)=B q^{-4}$, is observed in high- $q$ regions, where $q$ is the scattering wave vector defined as $(4 \pi / \lambda) \sin (\theta / 2) ; \lambda$ is the wavelength of the incident beam; $\theta$ is the scattering angle; and $B$ is a constant proportional to the average surface to volume ratio for the nanoparticles. The average spherical diameter can therefore be determined by $d_{p}=$ $6 \mathrm{~V} / \mathrm{S}$. The deviation from Porod's law at low $q$ regions can give $R_{g}$, the radius of gyration of the primary particles, using Guinier's law, $I(q)=G \cdot \exp \left(-q^{2} R_{g}{ }^{2} / 3\right)$, where $G$ is a constant proportional to the number density and average squared volume of the nanoparticles. Combining Porod's and Guinier's regimes, ratios between $B$ and $G$ can be utilized in charactering the polydispersity of the particles, i.e., fractal number and aggregate size.

Apart from static scattering methods, dynamic light scattering can also measure in situ particle sizes. In dynamic light scattering, particles with Brownian motion will give rise to fluctuations in the scattering field which can be correlated to the mean speed or alternatively the particle diffusion 
coefficient. The particle diffusion coefficient can then be related to a mean particle diameter. Zachariah et al. [526] measured the size of silica particles by dynamic light scattering in a counterflow diffusion flame. However, it was shown that the dynamic light scattering technique degrades rapidly as the signal strength declines.

Moreover, if we consider micro-sized droplets in spray flames, phase Doppler anemometry (PDA) can also provide information on droplet size and flow flux by measuring light-induced scattering or reflection from droplets. The phase difference of the scattered light from droplets in the intersection region of two laser beams can be related to the size and velocity of droplets. Pratsinis' group temporally measured the size and velocity of droplets in FSP [328][527], which exhibited good agreement with modelling results. PDA is restricted to spherical micro-sized particles and therefore has difficulties in extending to nanoparticles owing to the weak signal and aggregate morphologies.

\subsubsection{Raman spectroscopy}

Raman spectroscopy is a technique that can identify nanoparticle composition and crystallinity during flame synthesis. However, the inherently weak Raman effects generally limits the diagnostics to ex situ measurement on nanoparticles [528]. In addition, size confinement, temperature, and oxygen stoichiometry may influence the peak width and wavenumber shift for nanoparticles. In some work, Raman scattering is used to characterize the nanoparticle size, but other factors like anharmonicity, lattice parameter variation, and local electric field slightly influence the observed Raman shifts. In situ Raman diagnosis of nanoparticle aerosol formation during flame synthesis was first performed by Liu et al. [492] for charactering $\mathrm{TiO}_{2}$, revealing phase transitions from amorphous to anatase to rutile. In 
general, it is believed that the use of in situ Raman scattering for the composition of crystalline nanomaterials needs more investigation.

\subsubsection{Laser induced incandescence (LII)}

Laser induced incandescence (LII), a technique originally developed for soot diagnostics in combustion, takes advantage of the thermal effect of laser heating to bring particles to thousands of Kelvins in several nanoseconds and then to detect spatial and temporal near-blackbody emission from them [529][530]. The absolute radiation intensity is related to the volume fraction of particles, while the signal decay with time correlates to the particle size. Numerical models for energy and mass balance of particles are extremely important in determining particle size because of the complicated heat- and mass- loss mechanisms involved, such as conduction, radiation, and sublimation. These mechanisms involve important parameters, such as refractive-index function $E(m)$ and thermal and mass accommodation coefficients [531]. In extending LII investigation from near-blackbody soot to non-carbonaceous nanoparticles, in situ information of particle size can be accomplished, but there are two main challenges that need to be overcome: (i) new and different emission modes from irradiations due to electronically-excited gas-phase species from particles; and (ii) difficulty in modeling due to complicated absorption, emission, and accommodation coefficients of general particles.

Size distribution determination from LII signals, such as those on Ag and TiN [532], and $\mathrm{Fe}$ [533], depends heavily on the thermal balance model of particles, e.g. laser energy absorption by particles in the Rayleigh regime under grey-body assumption. The model proposed by Filippov et al. [532] considers convective heat transfer and material vaporization to/from the surroundings, but neglects 
thermal radiation in the thermal and mass balance equation of particles. Incandescence intensity correlates to particle size distribution via the solution of a Fredholm integral equation or by simply fitting parameters of certain size distributions. However, excessive assumptions and simplification of the LII models lead to discrepancies with TEM/DMA sampling. Eremen's work further indicated that the thermal accommodation factors are influenced by particle size [534][535] and bath gas [536], exposing that calibration work is extremely important for quantitative LII measurements. Lehre et al. [537] utilized two-color LII to obtain the temperature of particles, successfully reducing the uncertainties in the model. Cignoli et al. [538] utilized UV-light excitation in LII during flame synthesis of $\mathrm{TiO}_{2}$ to ensure the efficiency of absorption by the anatase lattice.

On the other hand, LII spectroscopy on metal particles of W, Fe, Mo, Ti [539], and Mo [540], along with metal oxides particles of $\mathrm{TiO}_{2}$ [541], all display obvious atomic emission of metal atoms superimposed on thermal irradiation of particles under high laser intensity. Electronic excitation from vaporization of particles [539] and laser induced emission from atoms in particles may cause misleading interpretations of LII signals, which may not correlate with the size or volume fraction of particles. In LII, the laser intensity in the probe volume needs to be controlled to ensure that the emission is pure incandescence from irradiation of the particles. Serendipitously, the unwanted atomic emission observed during LII experiments may reveal a novel phase-selective laser induced breakdown spectroscopy (PS-LIBS) regime.

\subsubsection{Phase-selective Laser induced breakdown spectroscopy}

As a novel laser diagnostic method developed under a collaboration between Li's lab at Tsinghua 
University and Tse's lab at Rutgers University, phase-selective laser induced breakdown spectroscopy (PS-LIBS) has shown the ability to be a diagnostic for gas-to-particle conversion [493][297], volume fraction measurement [296], and bandgap variation in mixed crystal structures [295]. Briefly, nanoparticles are selectively broken down into plasma, with no gas-phase breakdown due to the different thresholds between gas and particle phase, such that atomic spectroscopy can be made of only atoms originating from the particulate phase. Therefore, it can be applied to examine the gas-to-particle conversion process in flame aerosol synthesis [295][296][297], as well as sodium release during coal combustion [286]. When PS-LIBS signals reach saturation with laser intensity, the signal intensity is linear with volume fraction of particles, enabling quantitative measurement of volume fraction [296]. Two-dimensional imaging of particulate matter is further achieved [296][297], as seen in Fig. 19. Clearly, we can see a gas-to-particle conversion across the Bunsen laminar flame sheet, and the saturated signal intensity downstream reflects the conservative volume fraction during the collision-coalescence processes. Moreover, this map of atomic titanium emission perfectly images the gradient caused by diffusion and thermophoresis of nanoparticles, indicating that PS-LIBS can be used in studying fundamental transport properties of nanoparticles in an aerosol system.

Physically, PS-LIBS is believed to be caused by an absorption-ablation-excitation mechanism, which was recently clarified by Li and co-workers [298]. Rayleigh scattering measurements and further time scale analyses of the non-dimensional Fokker-Planck equation indicate that atomic emissions originate from an ablation process caused by laser absorption of conduction band electrons from one-photon or multi-photon ionization. Hence the signal intensity is sensitive to the variation of bandgap of particles, which is helpful in detecting the bandgap change during synthesis of mixed-composition 
nanoparticles, e.g. $\mathrm{V}_{2} \mathrm{O}_{5}-\mathrm{TiO}_{2}$ composite and other bandgap narrowing compositions in photocatalytic materials [294]. As shown in Fig. 20, adding vanadium significantly enhances the occurrence of atomic emission of titanium, especially at the initial stage of synthesis. This phenomenon is caused by the fact that the bandgap of $\mathrm{V}$-doped $\mathrm{TiO}_{2}$ particles $(2.3 \mathrm{eV})$ is smaller than that of pure $\mathrm{TiO}_{2}(3.2 \mathrm{eV})$. The larger signal intensity indicates that the bandgap variation of $\mathrm{TiO}_{2}$ starts at the burner outlet and therefore PS-LIBS is able to indicate when and where the bandgap variation begins, which is of great importance in designing the flame field for synthesizing nanoparticles with mixed components.

PS-LIBS is distinguished from other laser-based diagnostics by its different mechanisms of laser-matter interaction, as diagramed in Fig. 21, with different laser diagnostic processes marked in their regimes. When the laser intensity is relatively low $\left(<10^{-4} \mathrm{GW} / \mathrm{cm}^{2}\right)$, elastic or inelastic scattering is the dominant mechanism, corresponding to laser induced scattering (LIS) and Raman, respectively. At higher laser intensity beyond a critical threshold, particles are ablated. Notably, the ablation threshold is influenced by the photon energy and the bandgap of the particle. When the photon energy is not high enough to excite the electrons, higher laser intensity is needed to ensure the possibility of multi-photon excitation. The atomic emission of Ti has been observed for UV [538][541] laser irradiation of titania at less than $10^{-2} \mathrm{GW} / \mathrm{cm}^{2}$, while the threshold rises to $10^{-1} \mathrm{GW} / \mathrm{cm}^{2}$ at $532 \mathrm{~nm}$ wavelength and $1 \mathrm{GW} / \mathrm{cm}^{2}$ at $1064 \mathrm{~nm}$ wavelength. If the bandgap of the particle greatly exceeds the photon energy, the particle is only heated by the laser irradiation and no plasma forms, with the process situated in the LII regime. In the study of Mo particles by LII using 1064nm laser excitation [540], the combination of irradiation and ablation of particles produces a spectrum with atomic emission superimposed upon non-zero incandescence spectra. Incandescence radiation of material is believed to occur more easily when 
electrons are difficult to be excited in materials with high bandgap energy or non-metal materials like soot and $\mathrm{Si}$ [542]. When the laser intensity is high enough to breakdown all the matter (i.e. gas and solid) in the detection volume, conventional laser induced breakdown spectroscopy (LIBS) is achieved above a critical breakdown threshold, which can be used to detect the absolute elemental concentration after calibration. Amodeo et al. presented conventional LIBS measurement of $\mathrm{SiO}_{2}$ after guiding the aerosol to a certain volume [543], while Engle et al. used LIBS for detecting the existence of particles [495]. However, to our best knowledge, little conventional LIBS has been utilized in in situ on-line diagnosis of flame synthesis with spatial resolution because the strong breakdown process wipes off all information at the molecular level; and the millimeter-sized high-temperature plasma significantly disturbs the reaction process and synthesis products. In contrast to conventional LIBS, PS-LIBS exhibits a promising prospect of in situ on-line diagnostics of flame synthesis, in particular, for meeting the needs of complex doping synthesis of mixed oxide nanomaterials.

\section{Theory and modeling}

Because of the multi-scale characteristics on coupling transport phenomena (convection, diffusion, thermophoresis, and condensation), reaction kinetics, nucleation, and aerosol coagulation dynamics in flame synthesis, the theoretical framework for modeling and simulating poly-disperse nanoparticles comprises several models at different time and length scales, as schematically shown in Fig. 22. At the macroscopic continuum level, the population balance model (PBM) describes the evolution of the size distribution of nanoparticles during flame synthesis, which couples temperature, fluid velocity field, and species information (from CFD models) with reaction rates (from chemical kinetics models). Molecular 
dynamics (MD) simulation can further physically detail the parameters of collision and coalescence, and reactive MD simulation can provide information on heterogeneous reaction mechanisms, both of which can then be used in the continuum models.

\subsection{Time-scale analysis of flame synthesis process}

Before solving PBM coupled with both fluid dynamics and chemical reactions, time-scale analysis is a simple and fast approach to determine some of the most important physical factors, as well as estimating the final size of the nanoparticles, including multicomponent and doped ones. Here, time-scale analysis, i.e., calculation of characteristic times, is useful in gaining understanding of the whole flame synthesis system, and further in decoupling the multi-scale processes such as chemical reaction, Brownian coagulation, and sintering [10][21][323][324][326].

A typical time-scale analysis of flame synthesis in a premixed stagnation burner (as discussed in Section 2.5) is illustrated in Fig. 23. First, the chemical reaction time is calculated based on the kinetic parameters of the reactions. As an example, for $\mathrm{TTIP}^{-\mathrm{TiO}_{2}}$ conversion, hydrolysis reaction contributes the most to the production of $\mathrm{TiO}_{2}$ monomers, given the $\mathrm{OH}$-rich premixed flame. With the main reaction identified, the initial condition of PBM can be largely simplified. The ensuing nucleation process is usually omitted in the analysis because classical nucleation theory calculates the critical cluster size to be smaller than the dimension of a single monomer [22][545]. However, some investigators have argued that the homogenous nucleation rate is overestimated by classical nucleation theory [546].

The characteristic times of Brownian coagulation and sintering (also correspondingly termed 
collision and coalescence) are generally calculated to predict the final morphology of particles. When coagulation time is larger than sintering time, there is enough time for the primary particles to merge into a new particle before each collision. If sintering time is larger than coagulation time, there will not be enough time for complete sintering of the particles, and aggregates and agglomerates will form [324]. The characteristic time of collision can be calculated from the collision frequency, $\beta$. Generally, the free molecule assumption for low Kn number is adequate for flame-synthesized nanoparticles. Some results also imply that the sticking coefficient may be smaller than that predicted by standard kinetic theory at relatively low temperatures [547]. For the calculation of characteristic time of sintering, several empirical formulas can be found in the literature [19][270][548]. Nonetheless, sintering is very sensitive to temperature and particle size. Nontheless, if the flow time is well-selected, the coagulation process can be affected to control nanoparticle morphology and size.

In terms of flame synthesis of multi-element nano-products, studies have found a variety of morphologies under different species and flame environments [27][270]. So far, it is still difficult for PBM to describe the detailed interactions between different species in flames. In pioneering work by Zachariah and Dimitriou [549], they numerically calculated the characteristic time for scavenging in the $\mathrm{SiO}_{2}-\mathrm{TiO}_{2}$ system. Recently, $\mathrm{Li}$ and co-workers [324][326], extended the time-scale analysis to the $\mathrm{Pd}-\mathrm{TiO}_{2}$ supported catalyst system, showing effective deposition of Pd on the support surface, which in turn suppressed further growth of the Pd catalyst particles. 


\subsection{Continuum level approach: population balance modes}

\subsubsection{Basic PBM approach}

The population balance model (PBM) describes the population development of a system, and has been widely used in areas such as aerosol science, materials science, and cell biology [550]. Particularly in flame synthesis, the model defines the time derivative of particle concentration, in consideration of the birth and death (production and destruction) of new particles. Here, the basic equation is known as the aerosol general dynamic equation (GDE). Generally, it involves gas-to-particle conversion (nucleation), coagulation, and surface growth (condensation of monomers/surface reactions), and is given by:

$$
\frac{\partial n_{k}}{\partial t}=\left[\frac{\partial n_{k}}{\partial t}\right]_{\text {Nucleation }}+\left[\frac{\partial n_{k}}{\partial t}\right]_{\text {Coagulation }}+\left[\frac{\partial n_{k}}{\partial t}\right]_{\text {Surface growth }}
$$

where $k$ refers to the number of atoms/molecules in the particle.

Classical homogenous nucleation theory is the simplest way to describe the nucleation process in Eqn. (7.1). Accordingly, homogenous nucleation occurs when a particle reaches a critical size, containing $k^{*}$ atoms/molecules. Thus, the nucleation term generally has the form as given in Eqn. (7.2) and (6.3) [551]:

$$
\left[\frac{\partial n_{k^{*}}}{\partial t}\right]_{\text {Nucleation }}=\mathrm{A}^{*} \exp \left(-\frac{w^{*}}{k_{B} T}\right)
$$




$$
k^{*}=\frac{32 \pi}{3 v_{0}} \frac{\sigma^{3}}{|\Delta g|^{3}}
$$

In the above equations, $A^{*}$ and $w^{*}$ are the kinetic pre-factor and the work for forming a critical particle, respectively; $\sigma$ is surface tension; $v_{0}$ is the atomic or molecular volume; and $\Delta g$ is the Gibbs free energy per unit volume.

The coagulation term in Eqn. (7.1) can be derived from Brownian collision between particles, for which the governing equation is known as the Smoluchowski equation [552]

$$
\left[\frac{\partial n_{k}}{\partial t}\right]_{\text {Coagulation }}=\frac{1}{2} \sum_{\substack{i+j=k \\ i, j \neq 1}} \beta(i, j) n_{i} n_{j}-\sum_{i=2}^{\infty} \beta(i, \mathrm{k}) n_{i} n_{k}
$$

where $\beta$ is defined as the collision frequency. In the free molecule regime for flame synthesis of nanoparticles, $\beta$ can be calculated as

$$
\beta(i, j)=\left(\frac{3}{4 \pi}\right)^{1 / 6}\left(\frac{6 k T}{\rho}\right)^{1 / 2}\left(\frac{1}{v_{i}}+\frac{1}{v_{j}}\right)^{1 / 2}\left(v_{i}^{1 / 3}+v_{j}^{1 / 3}\right)^{2},
$$

where $v_{i}$ and $v_{j}$ are the volume of the particle $i$ and $j$, respectively; and $\rho$ is particle density.

The third term of Eqn. (7.1) is due to the condensation of monomers onto the particles and the evaporation of the monomers from the particles. Similar to the coagulation term, the surface term can be further expressed as:

$$
\left[\frac{\partial n_{k}}{\partial t}\right]_{\mathrm{Evap} / \text { Cond }}=\beta(1, k-1) n_{1} n_{k-1}-\beta(1, k) n_{1} n_{k}-\alpha_{k} s_{k} n_{k}+\alpha_{k+1} s_{k+1} n_{k+1},
$$


where $\beta$ is the collision frequency; $\alpha$ is the evaporative flux, which can be calculated by the Kelvin relation using the vapor pressure; and $s$ is the particle surface area [553].

Some special numerical approaches have been developed to solve the GDE equations to obtain the particle size distribution, as the equations are both nonlinear and partial integro-differential. These approaches include sectional methods with discretization and local approximation [554][555], moment based methods that can transform the equations into ordinary differential equations [556][557], and Monte-Carlo methods [558][559]. A comprehensive description and comparison of different methods can be found in a previous PECS review [560]. For easy use and for certain applications, Zachariah's group released a non-commercial GDE software package named NGDE, which is available for solving nucleation, surface growth, and coagulation problems [561][562].

\subsubsection{PBM coupled with fluid field}

Flame synthesis is generally featured as a one-step direct synthesis method, and the growth of the particles in a flame is therefore determined by the local fluid field. The residence time of particles in a flame, for example, can be as short as $5 \mathrm{~ms}$ in a premixed stagnation swirl flame (SSF) [112][324]. Also, the temperature profile undergoes dramatic change from ambient temperature to flame temperature. Therefore, the local fluid field will influence both physical and chemical processes involved in precursor reaction and particle growth, necessitating an update to the basic population balance model.

In a laminar flame environment, PBM coupled with fluid dynamics equations can still be solved by sectional or moment based methods, especially for nano-scale particles that should follow well the 
streamlines of the flow field. However, in turbulent flows, the problem is complex, due to the chaotic nature to be modeled [560]. The combination of moment based methods and CFD codes (LES/DNS) is the common approach to attack the problem, with certain assumptions and approximations, as shown in the early works by Pratsinis and co-workers [563][564]. In their comparisons, mono-disperse and one-dimensional models were found to show satisfactory results in simple systems [564]. A Gaussian quadrature-based moment method (QMOM) was developed by Rosner and co-workers [252][565][566]. It is a multivariate population balance model, based on Koch and Friedlander's bivariate model [567].

The moment method with interpolative closure (MoMIC), introduced from soot modelling, was also developed in these years [568]. For turbulent flames, a conditional quadrature method of moments (CQMOM) was proposed by Fox group to predict the $\mathrm{TiO}_{2}$ particle growth [569]. An overview of the latest development of PBM in turbulent flames can be found in their recent literature [570].

\subsubsection{PBM coupled with chemical reaction}

Along with the fluid field, kinetic reactions of the precursors should also be considered by the population balance models to describe properly particle growth in flame synthesis. These high temperature chemical reactions initiate the production of monomers, followed by particle nucleation and growth. Several typical precursor reactions are listed in Table 5. The precursor chemistry mainly involves pyrolysis, hydrolysis, and surface reactions. Taking $\mathrm{TTIP}^{-\mathrm{TiO}_{2}}$ conversion as an example, the decomposition/pyrolysis of TTIP, which has a large activation energy, is thermal-driven, while hydrolysis of TTIP, characterized by small activation energy, is radical-driven and often occurs in OH-rich flames. Fig. 24 (A) and (B) show the stationary points on the potential energy surface 
corresponding to the hydrolysis and thermal decomposition of TTIP and the main reaction route of TTIP hydrolysis. The activation energy barrier of TTIP hydrolysis is estimated to be ca. $10 \mathrm{kcal} / \mathrm{mol}$, while the barriers of thermal decomposition are much higher $(85-87 \mathrm{kcal} / \mathrm{mol})$ [571]. Based on the above quantum chemical computations and experimental data of Ti-containing intermediates concentrations in $\mathrm{H}_{22} / \mathrm{Ar}$ flame, Shmakov et al. [571] proposed the scheme of TTIP hydrolysis as presented in Fig. 24 (C). Therefore, radical-driven hydrolysis is the fastest reaction and usually dominates the precursor reaction of TTIP. Moreover, radical-driven reactions usually start at occur in the pre-heat zone upstream of the flame reaction zone, while thermal-driven reaction usually occurs in the reaction or post-flame zone, being highly dependent on temperature.

With the increasing computation power and the knowledge from quantum chemistry, researchers have started to adopt detailed chemical kinetic mechanisms combined with PBM. Menz and Kraft [579] employed an 8-step gas-phase mechanism and 6 heterogeneous reactions to simulate flame synthesis of silicon nanoparticles. Detailed chemistry in flame synthesis modeling still stands as a challenging topic, as many reactions are multi-phased and the reaction parameters are hard to be determined.

\subsection{Atomic level approach: Molecular dynamic simulation}

The atomic-level molecular dynamic simulation has been widely attempted to quantitatively characterize the collision-coalescence and other physical/chemical processes of nanoparticles like heterogenerious catalytic reaction of functional nanoparticles. In this work, the collision and coalescence of two approaching nanoparticles are intensively reviewed as follows. 


\subsubsection{MD simulations on particle collision/coagulation}

For example, the effect of long-range interactions on particle collision time is a long-existing problem in aerosol science. When the interaction between particles is on the order of the particle radius or longer, coagulation rate between particles are enhanced by increasing the capture radius of individual particles [580]-[584] and aggregates [585]. An enhancement factor $W_{\text {enh }}$ was proposed by Fuchs [586] to account for long-range interaction for the collision kernel, i.e.

$$
\beta=W_{e n h} \beta_{0}
$$

where $\beta$ is the collision kernel including the effects of long-range interaction; and $\beta_{0}$ is that only for Brownian coagulation given in Eqn. (7.5). Ouyang et al. reviewed a variety of expressions for the enhancement factor for both continuum and free-molecular regimes, for both charged and neutral particles [587]. Apart from these two types of long-range interactions, dipole-dipole interaction on enhancing the collision kernel should not be ignored, as nanoparticles can have permanent dipoles due to asymmetry of the crystalline structure or asymmetrical distribution of ions at their surface. These dipoles have been verified by experiments on CdSe [588] and ZnSe [589] and more recently by MD simulation on $\mathrm{TiO}_{2}[590]$.

Initially, Li and co-workers [590] compared van der Waals (vdW) and dipole-dipole forces between two $\mathrm{TiO}_{2}$ nanoparticles using $\mathrm{MD}$, as shown in Fig. 25 (a). The attractive dipole-dipole force is much

larger than the vdW force by several orders of magnitude at long distance. Thermal fluctuations of 
surface ions, especially at high temperatures, can lead to increasing fluctuation of the instantaneous dipole direction and finally a decrease in magnitude of the time-averaged dipole moment. They further applied MD to quantify the role of dipole-dipole interaction by calculating the enhancement factor $W_{\text {enh }}$ during the Brownian collision of two neutral $\mathrm{TiO}_{2}$ nanoparticles in the free molecular regime [591]. Critical capture radii $r_{f}$ were examined at four characteristic dipole-moment directions/orientations. As shown in Fig. 25 (b), dipole-dipole interactions significantly augment the enhancement factor compared with vdW interactions. As the temperature increases, the enhancement factors regress to the values of $\mathrm{vdW}$ interactions because of the reduction of the particle dipole moments as a result of thermal fluctuations.

\subsubsection{MD simulations on particle sintering}

Upon collision of two particles, sintering (or coalescence) dominates the growth of the 'merged' particle and is responsible for determining its final morphology and dimensions. In physics, sintering of nanoparticles is a thermal process that involves mass transport of atoms at the nanoscale. Thus MD simulation is frequently applied to investigate the mass transport routes of sintering. Usually, classic sintering theory of micro-sized ceramic particles cannot be extended to nanoparticles because several different features exist during sintering at the nanoscale.

First, a high portion of surface atoms comprising the nanoparticles change the melting point and lead to size-dependent crystalline-core-amorphous-shell structures of nanoparticles. Various statistical values from MD simulation have been proposed to describe structures and properties of nanoparticles, as summarized in Table 6. Potential energy and Lindemann index have been used to describe the 
crystallization state of nanoparticles. As presented in Fig. 26, critical diameters for the phase transition of grain structures for different temperatures are predicted by MD. At small particle diameters, the melting point depends on not only the particle temperature (like for bulk material) but also the particle size. From a thermodynamic consideration, an inverse linear relationship between the difference in temperature and the particle diameter has been proposed, i.e.,

$$
\left(T_{m 0}-T_{m p}\right) \propto \frac{1}{d_{p}},
$$

where $T_{m 0}$ and $T_{m p}$ are the melting temperatures of the bulk material and the nanoparticle, respectively; and $d_{p}$ is the particle diameter. Moreover, the average coordination number [592] and local lattice orientation [593] reveal that an amorphous shell of $0.4 \sim 0.6 \mathrm{~nm}$ surrounds a crystalline core, as visualized in Fig. 26 (a). Such a crystalline-core-amorphous-shell structure exists due to the highly distorted surface of the nanoparticles. When the nanoparticle size is smaller than a critical diameter, the influence of the amorphous shell penetrates the entire particle, forming a different amorphous grain structure, as illustrated in Fig. 26 (b). The critical transition diameters from amorphous to crystalline-core-amorphous-shell structures for $\mathrm{TiO}_{2}$ nanoparticles at different temperatures are shown by the red circles of Fig. 26, based on MD results. As such, different size-dependent structures of nanoparticles can lead to different coalescence processes; and nucleation or recrystallization may be involved during the growth of nanoparticles, which is significantly different from that for conventional sintering.

Second, different sintering mechanisms dominate for different kinds of nanoparticles. Surface 
diffusion and grain boundary diffusion were observed to be the main mass transport routes for particles with crystal structures, while viscous diffusion was dominant for amorphous particles. In the MD studies, several statistical values like surface area, gyration radius, ratio of moment of inertia, and shrinkage have been proposed to directly characterize the coalescence of the two nanoparticles into a single larger one. A typical sintering process between two $3 \mathrm{~nm}$ crystalline-core-amorphous-shell nanoparticles at $1573 \mathrm{~K}$ is characterized by temperature and normalized surface area, as shown in Fig. 27. The coalescence (sintering) process can be divided into four stages: (i) before contacting, the two particles rotate with a small angle to adjust their lattice planes (shown in snapshots A and B and regarded as the oriented attachment mechanism, which has also been discovered in the sintering processes of $\mathrm{Au}$ [594][595] and $\mathrm{Cu}$ [596] particles); (ii) a sintering neck forms between two particles and grows rapidly, accompanied by rapid surface reduction and temperature increase (shown in snapshot $\mathrm{C}$ ), which is controlled by surface diffusion; (iii) a grain boundary forms at the center of the two crystal cores, with further surface reduction (shown in snapshot D), which is controlled by both grain boundary diffusion and surface diffusion; and finally (iv) the particle shape transforms from ellipsoid to sphere. The sintering process between two amorphous particles is relatively simple, as its coalescence behaves similar to a fusion process between liquid droplets, controlled mainly by viscous diffusion. It should be noted that in all sintering processes, the high ratio of surface energy to total energy results in non-negligible heat release and temperature rise (with positive feedback) during sintering [597].

In the phenomenological Koch-Friedlander model,

$$
\frac{d a}{d t}=-\frac{1}{\tau}\left(a-a_{f}\right)
$$


where $a$ is the surface area during sintering; $a_{f}$ is the surface area after final coalescence; and $\tau$ is the characteristic sintering time. The aforementioned complicated mechanisms present difficulties in deriving a theoretical sintering time, except for some typical cases like the viscous-controlled sintering time of amorphous particles, which can be expressed as $t=\eta d_{p} / \sigma[598]$ or the solid-state sintering time ${ }_{s}$, which can be expressed by $t=3 k_{b} T v_{p} /\left(64 \sigma D v_{m}\right)$ [599]. Molecular simulation then provides an empirical equation after fitting the surface variation line.

Third, sintering can induce nucleation and recrystallization of nanoparticles. In MD simulation, $\mathrm{Li}$ and co-workers discovered a unique phase in the sintering of two $3 \mathrm{~nm}$ anatase nanoparticles [593], and Korparde et al. detected phase transformation in multi-particle sintering with multiple phases [600]. Thermodynamically, the free energy of a particle at nanoscale is not only dependent on temperature but on particle size as well. Naiker et al. [601] and Banfield et al. [602] quantified the effect of surface energy on the stability of $\mathrm{TiO}_{2}$ nanoparticles. Phase transition may occur when particle size increases during sintering. For example, for $\mathrm{TiO}_{2}$ particles, anatase phase is less stable than rutile phase for larger particles [479][603]; and amorphous particles may transform into crystalline-core-amorphous-shell nanoparticles during particle growth. Therefore, compared with the geometrical change at the last stage, crystal structure change can lead to significant temperature increase as shown in Fig. 27. Zhou and Fichthorn used MD simulations to probe kinetics of the transformation in individual anatase nanocrystals as well as in nanocrystal aggregates [604]. However, the details of sintering-induced phase transition in physics is still unclear in terms of whether or not sintering facilitates recrystallization and nucleation kinetically. Further investigation, particularly for phase transition of mixed metal oxides, is 
needed to be addressed in the future.

Different from collision-coalescence of nanoparticles in flame synthesis, sintering or thermal deactivation of catalyst supported immobile nanoparticles can be attributed to Ostwald Ripening [605][606], which involves inter-particle transport of mobile species with larger particles growing at the expense of smaller particles due to differences in surface energy. Ostwald ripening is a near-equilibrium process of aging, redistribution, or coarsening of matter in various areas [607][608]. It usually takes several hours to transpire for immobile solid particles, and thus its characterization is beyond the current ability of MD simulations. Ostwald ripening in this area has only been investigated by MD simulation in cases of liquid argon clusters in the high-pressure vapor phase [608].

\subsection{Mesoscopic approach: Lagrangian particle method}

Between the scales of Eulerian continuum approach and Lagrangian MD simulations, a mesoscopic approach lies in the description of flame aerosol system at the "discrete” particle level. The PBM-based continuum approach cannot describe the evolution of morphologies and structures of particle agglomerates or aggregates, whereas the MD simulations can do so but are constrained by the number of primary particles due to huge computational expense. Discrete-element method (DEM), as one of the typical Lagrangian particle methods sharing similar methodology with MD but fixed at the particle level, is suitable for direct descriptions of nanoscale particle dynamics. The well-developed DEM framework for micro-sized particles or bulk grains, which is integrated with CFD of both electric and flow fields, entirely incorporates particle-particle interactions, particle-particle fluid interactions and particle-particle 
electric interactions [609], DEM for nanoscale particles only began in recent years, and a tentative review on nanoscale dynamics can be found in literature [610]. As for soft-agglomerates in which the aforementioned van der Waals forces dominate interparticle interactions in the near-contact zone (i.e, short-range), the accuracy of the continuum models, based on classic adhesive contact theory, are validated by several researchers using MD to simulate the atomic interactions in nanoparticle collision problems [611]-[612]. The MD simulation results for the normal contact forces are compared to continuum theory predictions obtained by the Maugis-Dugdale, JKR (Johoson-Kendall-Roberts), and DMT (Derjaguin-Muller- Toporov) adhesive contact models. The Maguis-Dugdale model exhibits excellent agreement with MD results for 'large' nanoparticles ( $>20 \mathrm{~nm})$ [612], while the DMT model obtains reasonable agreement for 'small' nanoparticles, in a range of 1.95-8.24 nm [612]. Considering a normal force model similar to the DMT model as well as the related frictional and dissipation forces models (as seen in detail in [609]), a time-driven, soft sphere DEM was developed by Higashitani et al. [614] and Eggersdorfer et al. [615] for simulation of the restructuring and fragmentation of soft-agglomerates of nanoparticles under a shear flow. A much simpler event-driven, hard sphere DEM was applied by the same investigators to describe for the growth and structure of particles undergoing agglomeration/coagulation (in absence of coalescence/sintering) in both the free molecular and the continuum regimes by combining with Langevin dynamics method [616]. For hard aggregates in which the strong sintering bonds dominate the short-range interactions between particles, the event-driven DEM has also been developed to incorporate the sintering rate laws of various regimes including viscous-flow, lattice diffusion, and grain boundary diffusion [617]. Nevertheless, a hysteric sintering contact model that was proposed by Luding can be referred to constitute the time-driven DEM 
simulations on the reconstructing of particle aggregtes [618]. Generally speaking, time-driven, soft-sphere DEM is still neither well defined for describing the dynamics of contacting nanoparticles along both normal and tangential directions, nor convincingly validated by the experiments, including its complex coupling, across several orders of different time scales, with the CFD simulation for fluids.

\section{Conclusions and future directions}

Flame synthesis, in the past two decades, has not only proven to be one of the most scalable and economical technologies for producing well-controlled oxide nanostructured materials (covering most elements in the periodic table), but also been demonstrated to be a low cost fabrication method of functional thin films and nano-devices.

The various burner types, with vapor-fed or liquid-fed precursors, are summarized and compared. The co-flow jet burner had been widely used for large-scale commercial production, whereas flat flame burners, either Hencken (multi-element diffusion) or McKenna (premixed), are excellent tools for performing fundamental studies because of uniform temperature and flow fields. More recently, the flame spray pyrolysis burner has shown its advantage for producing nanomaterials nearly covering all elements in periodic table, along with facile doping of two or three elements during the synthesis. The premixed stagnation burner with a temperature-controlled substrate is suitable for precisely producing ultrafine nanoparticles at atomic accuracy as well as fabricating various kinds of functional thin-films, i.e., granular, columnar and mixed ones by controlling time scales of key sub-processes.

The progress on doping synthesis of mixed metal-oxide nanocatalysts, photocatalysts, sensing nanomaterials, and nanophosphors are reviewed from a point of view of morphological structures of 
nanocomposites. A diagram for the doping synthesis of these nanocomposites with desired structures, i.e., heterogeneously supported structure, homogeneously crystalline structure, and crystalline-core-amorphous-shell structure, is presented. Four key factors, including temperature, reaction pathway, burner type, and miscibility (in short as "TRBM"), constitute the parameters of this diagram. On the basis of flame aerosol synthesis of carbon nanostructures with different dimensions, the investigations on carbon-metal oxide nanocomposites by using flame spray pyrolysis are on the way.

As for diagnoses of flame synthesis processes, both in situ methods based on laser probing and $e x$ situ methods based on sampling need basic understanding of physical processes during application, i.e. aerosol dynamics during sampling and laser-matter interaction during laser excitation. Particular in this review, we summarize some representative laser-based diagnostics in a map of photon-bandgap versus laser fluence, which illustrates that laser-matter interactions at various levels reflect certain information of the particles. Especially, in contrast to other diagnostics, the novel PS-LIBS technique exhibits a promising prospect of in situ on-line diagnosis of flame synthesis, since it can well meet the needs of complex doping synthesis of mixed oxide nanomaterials.

To provide theoretical analysis of the complexities involved in flame synthesis at different levels, various approaches are employed, from the continuum approach, i.e. PBM, to the nanoscale approach, i.e. MD simulation. PBM is usually coupled with chemical reactions and fluid flow to give complete macroscopic simulation of the flame synthesis process. MD studies of collision, coalescence, and heterogeneous reaction are also reviewed. More fundamental studies to provide experimental data to verify these models at multiple scales should be conducted. For example, cluster measurement techniques are just now being applied with fast DMA systems coupled to mass spectrometers. The early 
stages of cluster formation will help bridge the gap between gas-phase and particle-phase measurements using a variety of spectroscopic techniques. As stated, such techniques will also help in the verification of cluster formation and growth models. In general, time-scale analysis, i.e., calculation of characteristics times, is very helpful for understanding the whole system and identifying the most important mechanism(s).

Despite fruitful development of flame synthesis in the past 20 years, the challenges, and more appropriately, the opportunities are still huge. Rather than just demonstration of flame synthesis to produce single- or multi-component functional materials of elements in the periodic table, more attention should be paid to explore the underlying physiochemical mechanisms that govern the complex formation of nanocomposite materials at the atomic level, and then to "upwardly" optimize the process design and to scale up reactors. Moreover, clarification of related mechanisms, e.g., doping mechanism or deposition mechanism, is needed for nanocomposite synthesis as well as precise fabrication of functional devices. Looking back to the research needs/challenges given in the introduction, the prospective work lies in following aspects accordingly:

(1) Atomic-precision controlling on the morphologies/properties of nanoparticles. While there are advanced methods that can measure particle size down to sub-nanometer ranges, the real time composition measurement still remains a challenge. For instance, in situ non-intrusive optical diagnostics, in combination with ex situ sampling measurements, for both gaseous precursors and nascent particles are greatly needed to further reveal several important physical processes that are still not clear, i.e., gas-to-particle formation and collision-coalescence (coagulation). The nucleation mechanism, either homogeneous or heterogeneous, of nano-clusters should be hierarchically studied by 
utilizing quantum and statistical mechanics and then validated by experiments; meanwhile, the cross-linking between MD simulation and continuum collision/sintering models should be established. Further, from a point of view of reactor design, the development of the stagnation flame configuration, the advanced quenching technology for jet flames, and the novel spray technique (e.g., reactive spray, electrospray) enables us to control the synthesis of nanoparticles at the atomic level, but the integration and optimization of these techniques still require development.

(2) Scalable and economical manufacturing of nanostructured materials. For good reactor design and process optimization, multi-scale modeling of chemical reactions, fluid flows, flame dynamics, particle dynamics, etc., during flame synthesis are needed to predict the key parameters for controlling the process, presenting a numerical challenge of various time scales across several orders of magnitudes. The corrected models for particle dynamics by using the atomic-level approaches, e.g. MD simulation, shall be included into macroscopic CFD simulations, aiding optimization and scale-up of flame synthesis processes. Two diffusion flame configurations, flame spray pyrolysis, and co-flow flame reactors, exhibit advantages at the industrial application level. However, on-line diagnoses on these kinds of reactors are urgently needed to control the scaled systems with respect to nanomaterial characteristics and to validate the multi-scale modeling of flame synthesis. In particular, the laser diagnostics on the atomization and sprays are of considerable interest, despite being a significant challenge.

(3) Complex doping to produce functional nanocomposites. The measurement and prediction of formation of complex nanocomposites, either mixed metal oxides or carbon-metal oxide, are more challenging than that of a single component. Indeed, in a nanocomposite synthesis process, the effects of 
tracer species on doped nanoparticles (i.e. doping mechanism), during either gas phase transformation or particle phase growth, are still not well theoretically studied. Characteristic sintering rates of multicomponent nanoparticles are urgently needed to be quantified not only at the atomic level by using MD simulation, but also at the mesoscopic particle level by using kinetic theory of grains. Excitingly, on-line in situ laser diagnostics of doping processes in flame synthesis by observing the changing band-gap of mixed oxide nanomaterials may become reliable given the rapid development of phase-selective LIBS and other optical methods in the past five years. The validation and verification between measurement and theoretical prediction on the doping mechanism should be conducted in a near future.

(4) Direct fabrication of flame-made materials into functional devices. The fabrication of functional devices viz. one-step flame aerosol deposition process has become of great interest in recent years due to the minimization of process steps. Compared with the aforementioned in-flame behaviors of nanoparticles, the diagnostics and modeling of nanoparticles in the deposition layer and on the substrate are much more challenging. The difficulty of on-line optical diagnostics arises from the thin boundary layer, the measurement noise induced by the wall, etc. Then, from a theoretical point of view, highly coupled processes of spray pyrolysis, flame synthesis, and particle deposition in a narrow domain greatly increase the difficulty of numerical simulation, apart from the complex geometry induced by the deposition masks or substrates. Actually, the flame aerosol deposition research is at the beginning stage, but will open a whole new realm of flame synthesis.

(5) Building theoretical modelling across several scales and validating by in situ diagnostics data. Basically, for the above four flame aerosol systems with different purposes, a generalized framework on 
multi-scale, multi-domain models are needed to overcome challenges posed by the highly nonlinear multi-scale response of various kind of flame systems. Nevertheless, the multi-field effects such as the electric, fluid, chemical, and thermal effects for both gas and particle phases should be considered in-parallel. To achieve this, the mesoscopic approach like DEM and other Lagrangian particle methods, which can bridge atomic-level MD simulations and PBM-based continuum model predictions, is of key importance but still relatively scarce. Moreover, the validation of the cross-scale hierarchical models (shown in Fig. 22) by in situ data from advanced diagnostics on gas, liquid, and particle phases is very difficult. Without the interpretation of the hierarchical models on multi-physics transport phenomena, the experimental data have little meaning.

Generally, potential application of flame-made materials and devices will determine their prospects. In our eyes, these applications should cut across many fields, beyond just energy-related areas, with joint interests among combustion, aerosol, and materials science communities. For example, catalytically-assisted homogeneous combustion of hydrocarbons using flame-made metal-support catalysts may allow the abatement of unburned hydrocarbons in conventional combustion engines and furnaces for emissions control. Flame-coated thin films may be helpful in solving carbon deposition problems in fuel spraying processes. Scalable flame synthesis of photocatalysts for solar-energy conversion and energy storage materials have started but need more research and development to become viable manufacturing routes. The nanocomposites of carbon materials and inorganic metal oxides through a single-step flame spray pyrolysis are of great relevance to many disciplines and fields, extending to medicine, electronics, and catalysis. Finally, it must be stated that significant empirical and mechanistic understanding have guided the development of flame aerosol systems. Meanwhile, 
developments in measurement, modeling, and integration with process control will further enable the development of flame aerosol reactor systems to produce the nanomaterials and devices that will impact society.

\section{Acknowledgements}

This work is mainly funded by the National Natural Science Funds of China (Nos. 51176094, 51390491) and by the National Key Basic Research and Development Program (No. 2013CB228506), and partially by the U.S. National Science Foundation and Office of Army Research for co-author S.D.T. The authors are particularly grateful to Prof. Paul Ronney at USC and Prof. Hai Wang at Stanford for organizing the NSF Workshop on Grand Challenges in Combustion. We acknowledge Prof. Sotiris Pratsinis and Dr. Karsten Wegner from ETH Zurich, Prof. Lutz Mädler from Bremen, Prof. Xiaolin Zheng from Stanford, Prof. Jun Huang from Sydney, Prof. Marshall Long and Prof. Daniel Rosner from Yale, Prof. Zhongshan Li from Lund, Prof. Michael Renfro from Connecticut, Prof. Christof Schulz from Duisburg-Essen, Prof. Stefan Will from Erlangen, Prof. Bernard Kear from Rutgers, Prof. C. K. Law from Princeton, and Prof. Qiang Yao and Prof. Yikang Pu from Tsinghua for their helpful and stimulating discussions. Special thanks are given to former and current students, Dr. Yiyang Zhang, Dr. Gang Xiong, Dr. Nafeng Wang, Mr. Yichen Zong, Miss Chenyang Liu, Mr. Yang Wang and Mr. Wenzheng Shi, for their help with the manuscript, tables, and figures. 


\section{Reference}

[1] Pratsinis SE. Flame aerosol synthesis of ceramic powders. Prog Energy Combust Sci 1998;24:197-219.

[2] Wooldridge MS. Gas-phase combustion synthesis of particles. Prog Energy Combust Sci 1998;24:63-97.

[3] Chen XB, Mao SS. Titanium dioxide nanomaterials: synthesis, properties, modifications, and applications. Chem Rev 2007;107(7):2891-2959

[4] Gupta AK, Gupta M. Synthesis and surface engineering of iron oxide nanoparticles for biomedical applications. Biomaterials 2005;26:3995-4021.

[5] Teo WY, Amal R, Mädler L. Flame spray pyrolysis: an enabling technology for nanoparticles design and fabrication. Nanoscale 2010;2:1324-1347.

[6] Lu YF, Fan HY, Stump A, Ward TL, Rieker T, Brinker CJ. Aerosol-assisted self-assembly of mesostructured spherical nanoparticles. Nature 1999;398:223-226.

[7] Brinker CJ, Lu YF, Sellinger A, Fan HY. Evaporation-induced self-assembly: nanostructures made easy. Adv Mater 1999;11(7):579-585.

[8] Boissiere C, Grosso D, Chaumonnot A, Nicole L, Sanchez C.Aerosol route to functional nanostructured inorganic and hybrid porous materials. Adv Mater 2011;23(5):599-623.

[9] Pratsinis SE. Aerosol-based technologies in nanoscale manufacturing: from functional materials to devices through core chemical engineering. AIChE J 2010;56:3028-3035.

[10] Zhang YY, Li SQ, Deng SL, Yao Q, Tse SD. Direct synthesis of nanostructured TiO2 films with controlled morphologies by stagnation swirl flames. J Aerosol Sci. 2012;44:71-82.

[11] Mädler L, Friedlander SK. Transport of nanoparticles in gases: overview and recent advances. Aerosol Air Qual Res 2007;7(3):304-342.

[12] Feng XD, Sayle DC, Wang ZL, Paras MS, Santora B, Sutorik AC, Sayle TXT, Yang Y, Ding Y, Wang XD, Her YS. Converting ceria polyhedral nanoparticles into single-crystal nanospheres. Science 2006;312:1504-1508.

[13] Ulrich GD. Flame synthesis of fine particles. Chem Eng News 1984;62:22.

[14] Ulrich GD. Theory of particle formation and growth in oxide synthesis flames. Combust Sci Technol. 1971;4:47-57.

[15] Formenti M, Juillet F, Meriaudeau P, Teichner SJ, and Vergnon PJ. Preparation in a hydrogen-oxygen flame of ultrafine metal oxide particles. Oxidative properties toward hydrocarbons in the presence of ultraviolet radiation. Colloid Interface Sci.1972;39:79.

[16] Brezinsky K. Gas-phase combustion synthesis of materials. Proc Combust Inst 1996;26(2):1805-1816.

[17] Merchan-Merchan W, Saveliev AV, Kennedy L, Jimenez WC. Combustion synthesis of carbon nanotubes and related nanostructures. Prog Energy Combust Sci 2010;36(6):696-727.

[18] Friedlander SK, Pui DY. Emerging issues in nanoparticle aerosol science and technology. J Nano Res 2004;6(2):313-320.

[19] Xiong Y, Pratsinis SE. Formation of agglomerate particles by coagulation and sintering-Part I. A two-dimensional solution of the population balance equation. J Aerosol Sci 1993;24:283-300.

[20] Spicer PT, Chaoul O, Tsantilis S, Pratsinis SE. Titania formation by $\mathrm{TiCl}_{4}$ gas-phase oxidation, surface growth and coagulation. J Aerosol Sci 2002;33:17-34. 
[21] Xing Y, Köylü ÜÖ, Rosner D E. Synthesis and restructuring of inorganic nano-particles in counterflow diffusion flames. Combust Flame 1996;107:85-102.

[22] Roth P. Particle synthesis from flames. Proc Combust Inst 2007;37:1173-1188.

[23] Hawa T, Zachariah MR. Molecular dynamics study of particle-particle collisions between hydrogenpassivated silicon nanoparticles. Phys Rev B 2004;69:035417.

[24] Zachariah MR. Nanoparticle aerosol dynamics and particle technology. Lecture Notes, University of Maryland, 2010.

[25] Strobel R. Aerosol flame synthesis of supported metal particles and their catalytic applications. $2006 \mathrm{Ph} \mathrm{D}$ Dissertation, ETH, Switzerland.

[26] Strobel R., Baiker A., Pratsinis SE. Aerosol flame synthesis of catalysts. Adv Powder Technol 2006;17:457-480.

[27] Strobel R, Pratsinis SE. Flame aerosol synthesis of smart nanostructured materials. J Mater Chem 2007; 17:4743-4756.

[28] Schimmoeller B, Pratsinis SE, Baiker A. Flame Aerosol Synthesis of Metal Oxide Catalysts with Unprecedented Structural and Catalytic Properties. Chem Cata Chem 2011;3:1234-1256.

[29] Sokolowski M, Sokolowska A, Michalski A, Gokieli B. The "in- flame-reaction" method for $\mathrm{Al}_{2} \mathrm{O}_{3}$ aerosol formation. J Aerosol Sci 1977;8:219-230.

[30] Bickmore CR, Waldner KF, Treadwell DR, Laine RM. Ultrafine Spinel Powders by Flame Spray Pyrolysis of a Magnesium Aluminum Double Alkoxide. J Am Ceram Soc 1996;79:1419-1423.

[31] Bickmore CR, Waldner KF, Baranwal R, Hinklin T, Treadwell DR, Laine RM. Ultrafine Titania by Flampe Spray Pyrolysis of a Titanatrane complex. J Eur Ceram Soc 1998;18:287-297.

[32] Sutorik AC, Neo, SS, Treadwell DR, Laine RM. Synthesis of ultrafine $\beta$-alumina powders via flame spray pyrolysis of polymeric precursors. J Am Ceram Soc 1998;81:1477-1480

[33] Baranwal R, Villar MP, Garcia R, Laine RM. Flame spray pyrolysis of precursors as a route to nano-mullite powder: Powder characterization and sintering behavior. J Am Ceram Soc 2001;84:951-961.

[34] Marchal J, John T, Baranwal R, Hinklin T, Laine RM. Yttrium aluminum garnet nanopowders produced by liquid-feed flame spray pyrolysis (LF-FSP) of metalloorganic precursors. Chem Mater. 2004;16:822-831.

[35] Kim M, Laine RM. One-Step Synthesis of Core-Shell (Ce0.7Zr0.3O2)x(A12O3)1-x[(Ce0.7Zr0.3O2)@A12O3] Nanopowders via Liquid-Feed Flame Spray Pyrolysis (LF-FSP) J Am Chem Soc 2009;131:9220-9229.

[36] Kim S, Gislason, JJ, Morton RW, Pan XQ, Sun HP, Laine RM. Liquid-feed flame spray pyrolysis of nanopowders in the alumina-titania system. Chem Mater 2004;16:2336-2343.

[37] Laine RM, Marchal J, Sun HP, Pan XQ. A new $\mathrm{Y}_{3} \mathrm{Al}_{5} \mathrm{O}_{12}$ phase produced by liquid-feed flame spray pyrolysis (LF-FSP). Adv Mater 2005;17:830-833.

[38] Hinklin TR, Azurdia J, Kim M, Marchal JC, Kumar S, Laine RM. Finding spinel in all the wrong places. Adv Mater 2008;20:1373-1375.

[39] Karthikeyan J, Berndt CC, Tikkanen J, Wang JY, King A H, Herman H. Nanomaterial powders and deposits prepared by flame spray processing of liquid precursors. Nanostruct Mater 1997;8:61-74.

[40] Tikkanen J, Gross KA, Berndt CC, Pitkanen V, Keskinen J, Raghu S, Rajala M, Karthikeyan J. Characteristics of the liquid flame spray process. Surf Coat Technol 1997;90:210-216.

[41] Mädler L, Kammler HK, Mueller R, Pratsinis SE. Controlled synthesis of nanostructured particles by flame 
spray pyrolysis. J Aerosol Sci 2002;33:369-389.

[42] Mädler L, Stark WJ, Pratsinis SE. Flame-made ceria nanoparticles. J Mater Res 2002;17:1356-1362.

[43] Mädler L, Pratsinis SE. Bismuth oxide nanoparticles by flame spray pyrolysis. J Am Ceram Soc, 2002;85:1713.

[44] Tani T, Mädler L, Pratsinis, SE. Homogeneous ZnO nanoparticles by flame spray pyrolysis. J Nanoparticle Res 2002;4:337-343.

[45] Tani T, Mädler L, Pratsinis SE. Synthesis of zinc oxide/silica composite nanoparticles by flame spray pyrolysis. J Mater Res 2002;37:4627-4632.

[46] Lengyel M, Atlas G, Elhassid D, Zhang XF, Belharouak I, Axelbaum RL.Effects of Lithium Content and Surface Area on the Electrochemical Performance of $\mathrm{Li}_{1.2} \mathrm{Mn}_{0.54} \mathrm{Ni}_{0.13} \mathrm{Co}_{0.13} \mathrm{O}_{2}$. J Electrochem. Soc 2014;161:A1023-A1031.

[47] Yi EY, Wang WM, MohantyS, Kieffer J, Tamaki R, Laine RM. Materials that can replace liquid electrolytes in $\mathrm{Li}$ batteries: Superionic conductivities in $\mathrm{Li}_{1.7} \mathrm{Al}_{0.3} \mathrm{Ti}_{1.7} \mathrm{Si}_{0.4} \mathrm{P}_{2.6} \mathrm{O}_{12}$. Processing combustion synthesized nanopowders to free standing thin films. J Power Sources 2014;269:577-588.

[48] Choi SH, Kim JH, KoYN, Yang KM, KangYC. Preparation and electrochemical properties of glass-modified $\mathrm{LiCoO}_{2}$ cathode powders. J Power Sources 2013;244:129-135.

[49] Chiang CY, Shin Y, Ehrman S. Li Doped CuO Film Electrodes for Photoelectrochemical Cells. J Electrochem Soc 2012;159:B227-B231.

[50] Zhang XF, Zheng HH, Battaglia V, Axelbaum RL. Electrochemical performance of spinel $\mathrm{LiMn}_{2} \mathrm{O}_{4}$ cathode materials made by flame-assisted spray technology. J Power Sources 2011;196:3640-3645

[51] Ernst FO, Kammler HK, Roessler A, Pratsinis SE, Stark WJ, Ufheil U, Novak P. Electrochemically active flame-made nanosized spinels: $\mathrm{LiMn}_{2} \mathrm{O}_{4}, \mathrm{Li}_{4} \mathrm{Ti}_{5} \mathrm{O}_{12}$ and $\mathrm{LiFe}_{5} \mathrm{O}_{8}$. Mater Chem Phys 2007;101:372-378.

[52] Height MJ, Mädler L, Pratsinis SE, Krumeich F. Nanorods of ZnO made by flame spray pyrolysis. Chem Mater 2006;18:572-578.

[53] Grass RN, Stark WJ. Flame synthesis of calcium-, strontium-, barium fluoride nanoparticles and sodium chloride. Chem Commun 2005;13:1767-1769.

[54] Brunner TJ, Grass RN, Stark WJ. Glass and bioglass nanopowders by flame synthesis. Chem Commun 2006;13: 1384-1386.

[55] Koirala R, Buchel R, Pratsinis SE, Baiker A. Oxidative coupling of methane on flame-made $\mathrm{Mn}-\mathrm{Na}_{2} \mathrm{WO}_{4} / \mathrm{SiO}_{2}$ : Influence of catalyst composition and reaction conditions. Appl Catal A-Gen 2014;484:97-107.

[56] Suzuki T, Ohishi Y, Tani T. Structural and fluorescence properties of Ni:MgO-SiO2 particles synthesized by flame spray pyrolysis. Mater Sci Eng B 2006;128:151-155.

[57] Tani T, Saeki S, Suzuki T, Ohishi Y. Chromium-doped forsterite nanoparticle synthesis by flame spray pyrolysis. J Am Ceram Soc 2007;90:805-808.

[58] Roy S, van Vegten N, Baiker A. Single-step flame-made Pt/MgAl $\mathrm{O}_{4}$ - A NOx storage-reduction catalyst with unprecedented dynamic behavior and high thermal stability. J Catal 2010;271:125-131.

[59] Roy S, van Vegten N, Maeda N, Baiker A. NOx storage and reduction over flame-made $\mathrm{M} / \mathrm{MgAl}_{2} \mathrm{O}_{4}(\mathrm{M}=\mathrm{Pt}$, Pd, and Rh): A comparative study. Appl Catal B-Environ 2012;119:279-286.

[60] Haider P, Baiker A. Gold supported on Cu-Mg-Al-mixed oxides: strong enhancement of activity in aerobic alcohol oxidation by concerted effect of copper and magnesium. J Catal 2007;248:175-187. 
[61] Haider P, Grunwaldt JD, Baiker A. Gold supported on Cu-Mg-Al-mixed oxides: Relation between surface properties and behavior in catalytic aerobic oxidation of 1-phenylethanol. Catal Today 2009;141:349-354.

[62] van Vegten N, Maciejewski M, Krumeich F, Baiker A. Structural properties, redox behaviour and methane combustion activity of differently supported flame-made Pd catalysts. Appl Catal B-Environ 2009;93:38-49.

[63] Rossetti I, Fabbrini L, Ballarini N, Oliva C, Cavani F, Cericola A, Bonelli B, Piumetti M, Garrone E, Dyrbeck H, Blekkan EA, Forni L. V-Al-O catalysts prepared by flame pyrolysis for the oxidative dehydrogenation of propane to propylene. Catal Today 2009;141:271-281.

[64] Taylor NJ, Pottebaum AJ, Uz V, Laine RM. The bottom up approach is not always the best processing method: dense- $\mathrm{Al}_{2} \mathrm{O}_{3} / \mathrm{NiAl}_{2} \mathrm{O}_{4}$ composites. Adv Funct Mater 2014;24:3392-3398.

[65] Debecker DP, Schimmoeller B, Stoyanova M, Poleunis C, Bertrand P, Rodemerck U, Gaigneaux EM. Flame-made $\mathrm{MoO}_{3} / \mathrm{SiO}_{2}-\mathrm{Al}_{2} \mathrm{O}_{3}$ metathesis catalysts with highly dispersed and highly active molybdate species. J Catal 2011;277:154-163.

[66] Buchel R, Baiker A, Pratsinis SE. Effect of $\mathrm{Ba}$ and $\mathrm{K}$ addition and controlled spatial deposition of Rh in $\mathrm{Rh} / \mathrm{Al}_{2} \mathrm{O}_{3}$ catalysts for $\mathrm{CO}_{2}$ hydrogenation. Appl Catal A-Gen. 2014;477:93-101.

[67] Buchel R, StrobelR, Krumeich F, Baiker A, Pratsinis SE. Influence of Pt location on $\mathrm{BaCO}_{3}$ or $\mathrm{Al}_{2} \mathrm{O}_{3}$ during NOx storage reduction. J Catal 2009;261:201-207.

[68] Hannemann S, Grunwaldt JD, Lienemann P, Gunther D, Krumeich F, Pratsinis SE, Baiker A. Combination of flame synthesis and high-throughput experimentation: The preparation of alumina-supported noble metal particles and their application in the partial oxidation of methane. Appl Catal A-Gen. 2007;316:226-239.

[69] Strobel R, Grunwaldt JD, CamenzindA, Pratsinis SE, Baiker A. Flame-made alumina supported Pd-Pt nanoparticles: Structural properties and catalytic behavior in methane combustion. Catal Lett 2005;104:9-16.

[70] Strobel R, Stark WJ, Mädler L, Pratsinis SE, Baiker A. Flame-made platinum/alumina: structural properties and catalytic behaviour in enantioselective hydrogenation. J Catal 2003;213:296-304.

[71] Strobel R, Pratsinis SE, Baiker A. Flame-made $\mathrm{Pd} / \mathrm{La}_{2} \mathrm{O}_{3} / \mathrm{Al}_{2} \mathrm{O}_{3}$ nanoparticles: thermal stability and catalytic behavior in methane combustion. J Mater Chem 2005;15:605-610.

[72] Dongil AB, Bachiller-Baeza B, Rodriguez-Ramos I, Guerrero-Ruiz A, Mondelli C, Baiker A. Structural properties of alumina- and silica-supported Iridium catalysts and their behavior in the enantioselective hydrogenation of ethyl pyruvate. Appl Catal A-Gen 2013;451:14-20.

[73] Huang J, Jiang YJ, van Vegten N, Hunger M, Baiker A. Tuning the support acidity of flame-made $\mathrm{Pd} / \mathrm{SiO}_{2}-\mathrm{Al}_{2} \mathrm{O}_{3}$ catalysts for chemoselective hydrogenation. J Catal 2011;281:352-360.

[74] Williams GR, Bayram SB, Rand SC, Hinklin T, Laine RM. Laser action in strongly scattering rare-earth-metal-doped dielectric nanophosphors. Phys Rev A 2002;65:013807.

[75] Schulz H, Stark WJ, Maciejewski M, Pratsinis SE, Baiker A. Flame-made nanocrystalline ceria/zirconia doped with alumina or silica: structural properties and enhanced oxygen exchange capacity. J Mater Chem 2003;13:2979-2984.

[76] Piumetti M, Bonelli B, Massiani P, Dzwigaj S, Rossetti I, Casale S, Armandi M, Thomas C, Garrone E. Effect of vanadium dispersion and of support properties on the catalytic activity of V-containing silicas. Catal Today 2012;179:140-148.

[77] Rossetti I, Fabbrini L, Ballarini N, Oliva C, Cavani F, Cericola A, Bonelli B, Piumetti M, Garrone E, Dyrbeck H, Blekkan EA, Forni, L. $\mathrm{V}_{2} \mathrm{O}_{5}-\mathrm{SiO}_{2}$ systems prepared by flame pyrolysis as catalysts for the oxidative dehydrogenation of propane. J Catal 2008;256:45-61. 
[78] Jossen R, Heine MC, Pratsinis, SE, Augustine SM, Akhtar MK. Thermal stability and catalytic activity of flame-made silica-vanadia-tungsten oxide-titania. Appl Catal B-Env 2007;69:181-188.

[79] Li D, Teoh WY, Selomulya C, Woodward RC, Amal R, Rosche B. Flame-sprayed superparamagnetic bare and silica-coated maghemite nanoparticles: Synthesis, characterization, and protein adsorption-desorption. Chem Mater 2006;18:6403-6413.

[80] Ramin M, van Vegten N, Grunwaldt JD, Baiker A. Simple preparation routes towards novel Zn-based catalysts for the solventless synthesis of propylene carbonate using dense carbon dioxide. J Mole Catal A-Chem 2006;258:165-171.

[81] Lee JS, Oh MH, Kumar P, Khanna A, Singh RK, Ranade MB. Mn-Doped $\mathrm{Zn}_{2} \mathrm{SiO}_{4}$ Phosphors Synthesis Using Flame Spray Pyrolysis. J Thermal Spray Technol 2011;20:1001-1008.

[82] Tricoli A, Graf M, Pratsinis SE. Optimal doping for enhanced $\mathrm{SnO}_{2}$ sensitivity and thermal stability. Adv Funct Mater 2008;18:1969-1976.

[83] Wang ZC, Jiang YJ, Hunger M, Baiker A, Huang J. Catalytic Performance of Bronsted and Lewis Acid Sites in Phenylglyoxal Conversion on Flame-Derived Silica-Zirconia. ChemCatChem 2014;6:2970-2975.

[84] Schulz H, Mädler L, Pratsinis SE, Burtscher P, Moszner N. Transparent nanocomposites of radiopaque, flame-made $\mathrm{Ta}_{2} \mathrm{O}_{5} / \mathrm{SiO}_{2}$ particles in an acrylic matrix. Adv Funct Mater 2005;15:830-837.

[85] Hannemann S, Grunwaldt JD, Krumeich F, Kappen P, Baiker A. Electron microscopy and EXAFS studies on oxide-supported gold-silver nanoparticles prepared by flame spray pyrolysis. Appl Surf Sci 2006;252:7862-7873.

[86] Finocchio E, Rossetti I, Ramis G. Redox properties of Co- and Cu-based catalysts for the steam reforming of ethanol. Int J Hydrog Energy 2013;8:3213-3225.

[87] Rossetti I, Biffi C, Bianchi CL, Nichele V, Signoretto M, Menegazzo F, Finocchio E, Ramis G, Di Michele A. $\mathrm{Ni} / \mathrm{SiO}_{2}$ and $\mathrm{Ni} / \mathrm{ZrO}_{2}$ catalysts for the steam reforming of ethanol. Appl Catal B-Environ. 2012;117:384-396.

[88] Qin X, Ju Y, BernhardS, Yao N. Europium-doped yttrium silicate nanophosphors prepared by flame synthesis. Mater Res Bull 2007;42:1440-1449.

[89] Beier MJ, Schimmoeller B, Hansen TW, Andersen JET, Pratsinis SE, Grunwaldt JD. Selective side-chain oxidation of alkyl aromatic compounds catalyzed by cerium modified silver catalysts. J Mole Catal -Chem 2010;331:40-49.

[90] Strobel R, Metz HJ, Pratsinis SE. Brilliant yellow, transparent pure, and $\mathrm{SiO}_{2}$-coated $\mathrm{BiVO}_{4}$ nanoparticles made in flames. Chem Mater 2008;20:6346-6351.

[91] Loher S, Stark WJ, Maciejewski M, Baiker A, Pratsinis SE, Reichardt D, Maspero F, Krumeich F, Gunther D. Fluoro-apatite and calcium phosphate nanoparticles by flame synthesis. Chem Mater 2005;17:36-42.

[92] Loher S, Schneider OD, Maienfisch T, Bokorny S, Stark WJ, Micro-organism-triggered release of silver nanoparticles from biodegradable oxide carriers allows preparation of self-sterilizing polymer surfaces. Small 2008;4:824-832.

[93] Lu H, Smirniotis PG, Ernst FO, Pratsinis SE. Nanostructured Ca-based sorbents with high $\mathrm{CO}_{2}$ uptake efficiency. Chem Eng Sci 2009;64:1936-1943.

[94] Osterwalder N, Loher S, Grass RN, Brunner TJ, Limbach LK, Halim SC, Stark WJ. Preparation of nano-gypsum from anhydrite nanoparticles: Strongly increased Vickers hardness and formation of calcium sulfate nano-needles. J Nanopart Res 2007;9:275-281. 
[95] Huber M, Stark, WJ, Loher S, Maciejewski M, Krumeich F, Baiker A. Flame synthesis of calcium carbonate nanoparticles. Chem Commun 2005;5:648-650.

[96] Wang JJ, Li SQ, Yan W, Tse SD, Yao Q. Synthesis of $\mathrm{TiO}_{2}$ nanoparticles by premixed stagnation swirl flames. Proc Combust Inst 2011;33:1925-1932.

[97] Teoh WY, Mädler L, Beydoun D, Pratsinis SE, Amal R. Direct (one-step) synthesis of $\mathrm{TiO}_{2}$ and $\mathrm{Pt} / \mathrm{TiO}{ }_{2}$ nanoparticles for photocatalytic mineralisation of sucrose. Chem Eng Sci 2005;60:5852-5861.

[98] Lopez A, Acosta D, Martinez AI, Santiago J. Nanostructured low crystallized titanium dioxide thin films with good photocatalytic activity. Powder Technol. 2010;202:111-117.

[99] Teleki A, Buesser B, Heine MC, Krumeich F, Akhtar MK, Pratsinis SE. Role of gas-aerosol mixing during in situ coating of flame-made titania particles. Ind Eng Chem Res 2009;48:85-92.

[100] Schimmoeller B, Delaigle R, Debecker DP, Gaigneaux EM. Flame-made vs wet-impregnated vanadia/titania in the total oxidation of chlorobenzene Possible role of VOx species. Catal Today 2010;157:198-203.

[101] Boningari T, Koirala R, Srnirniotis PG. Low-temperature catalytic reduction of NO by $\mathrm{NH}_{3}$ over vanadia-based nanoparticles prepared by flame-assisted spray pyrolysis: Influence of various supports. Appl Catal B-Environ 2013;140:289-298.

[102] Inturi SNR, Boningari T, Suidan M, Smirniotis PG. Visible-light-induced photodegradation of gas phase acetonitrile using aerosol-made transition metal (V, Cr, Fe, Co, Mn, Mo, Ni, Cu, Y, Ce, and Zr) doped $\mathrm{TiO}_{2}$. Appl Catal B-Environ 2014;144:333-342.

[103] Cai LL, Cho IS, Logar M, Mehta A, He JJ, Lee CH, Rao PM, Feng YZ, Wilcox J, Prinz FB, Zheng XL. Sol-flame synthesis of cobalt-doped $\mathrm{TiO}_{2}$ nanowires with enhanced electrocatalytic activity for oxygen evolution reaction. Phys Chem Chem Phys 2014;16:12299-12306.

[104] Teoh WY, Amal R, Mädler L, Pratsinis SE. Flame sprayed visible light-active $\mathrm{Fe}^{-\mathrm{TiO}_{2}}$ for photomineralisation of oxalic acid. Catal Today 2007;120:203-213.

[105] George S, Pokhrel S, Ji ZX, Henderson BL, Xia T, Li LJ, Zink JI, Nel AE, Mädler L. Role of Fe Doping in Tuning the Band Gap of $\mathrm{TiO}_{2}$ for the Photo-Oxidation-Induced Cytotoxicity Paradigm. J Am Chem Soc 2011;133:1270-11278.

[106] Siriwong C, Phanichphant S. Flame-made single phase $\mathrm{Zn}_{2} \mathrm{TiO}_{4}$ nanoparticles. Mater Lett 2011;65:2007-2009.

[107] Jung DS, Hong SK, Cho JS, Kang YC. Morphologies and crystal structures of nano-sized $\mathrm{Ba}_{1-\mathrm{x}} \mathrm{Sr}_{\mathrm{x}} \mathrm{TiO}_{3}$ primary particles prepared by flame spray pyrolysis. Mater Res Bullet 2008;43:1789-1799.

[108] Kazakevicius E, Tsekouras G, Michalow-Mauke KA, Kazlauskas S, Graule T. Electronic Conductivity Enhancement of ( $\mathrm{La}, \mathrm{Sr}) \mathrm{TiO}_{3}$ with Nb-Doping on B-Site. Fuel Cells 2014;14:954-960.

[109] Teleki A, Bjelobrk N, Pratsinis SE. Flame-made Nb- and $\mathrm{Cu}$-doped $\mathrm{TiO}_{2}$ sensors for $\mathrm{CO}$ and ethanol. Sens Actuator B-Chem 2008;130(1):449-457.

[110] Phanichphant S, Liewhiran C, Wetchakun K, Wisitsoraat A, Tuantranont A. Flame-Made Nb-Doped $\mathrm{TiO}_{2}$ Ethanol and Acetone. Sensors 2011;11:472-484.

[111] Park GD, Choi SH, Kang YC. Electrochemical properties of ultrafine $\mathrm{TiO}_{2}$-doped $\mathrm{MoO}_{3}$ nanoplates prepared by one-pot flame spray pyrolysis. RSC Adv 2014;33:17382-17386.

[112] Niu F, Li SQ, Zong YC, Yao Q. Catalytic behavior of flame-made $\mathrm{Pd} / \mathrm{TiO}_{2}$ nanoparticles in methane oxidation at low temperatures. J. Phys Chem C 2014;118:19165-19171. 
[113] Chiarello GL, Aguirre MH, Selli E. Hydrogen production by photocatalytic steam reforming of methanol on noble metal-modified $\mathrm{TiO}_{2}$. J Catal 2010;273:182-190.

[114] Chiarello GL, Ferri D, Selli E. Effect of the $\mathrm{CH}_{3} \mathrm{OH} / \mathrm{H}_{2} \mathrm{O}$ ratio on the mechanism of the gas-phase photocatalytic reforming of methanol on noble metal-modified $\mathrm{TiO}_{2}$. J Catal 2011;280:168-177.

[115] Tricoli A, Righettoni M, Pratsinis SE. Minimal cross-sensitivity to humidity during ethanol detection by $\mathrm{SnO}_{2}-\mathrm{TiO}_{2}$ solid solutions. Nanotechnology 2009;31:315502

[116] Purwanto A, Wang WN, Lenggoro IW, Okuyama K. Formation of $\mathrm{BaTiO}_{3}$ nanoparticles from an aqueous precursor by flame-assisted spray pyrolysis. J Eur Ceram Soc 2007;27:4489-4497.

[117] Chaisuk C, Wehatoranawee A, Preampiyawat S, Netiphat S, Shotipruk A, Panpranot J, Jongsomjit B, Mekasuwandumrong O. Preparation and characterization of $\mathrm{CeO}_{2} / \mathrm{TiO}_{2}$ nanoparticles by flame spray pyrolysis. Ceram Int 2011;37:1459-1463.

[118] Akurati KK, Vital A, Dellemann JP, Michalow K, Graule T, Fetti D, Baiker A. Flame-made $\mathrm{WO}_{3} / \mathrm{TiO}_{2}$ nanoparticles: Relation between surface acidity, structure and photocatalytic activity. Appl Catal B-Environ 2008;79:53-62.

[119] Teoh WY, Mädler L, Amal R. Inter-relationship between Pt oxidation states on $\mathrm{TiO}_{2}$ and the photocatalytic mineralisation of organic matters. J Catal 2007;251(2):271-280.

[120] Bubenhofer SB, Schumacher CM, Koehler FM, Luechinger NA, Grass RN, Stark WJ. Large-Scale Synthesis of PbS-TiO2 Heterojunction Nanoparticles in a Single Step for Solar Cell Application. J Phys Chem C 2012;116:16264-16270.

[121] Sel S, Duygulu O, Kadiroglu U, Machin NE. Synthesis and characterization of nano- $\mathrm{V}_{2} \mathrm{O}_{5}$ by flame spray pyrolysis, and its cathodic performance in Li-ion rechargeable batteries. Appl Surf Sci 2014;318:150-156

[122] Ng SH, Patey TJ, Buechel R, Krumeich F, Wang JZ, Liu HK, Pratsinis SE, Novak P. Flame spray-pyrolyzed vanadium oxide nanoparticles for lithium battery cathodes. Phys Chem Chem Phys 2009;11:3748-3755.

[123] Castillo NC, Heel A, Graule T, Pulgarin C. Flame-assisted synthesis of nanoscale, amorphous and crystalline, spherical $\mathrm{BiVO}_{4}$ with visible-light photocatalytic activity. Appl Catal B-Environ 2010;95:335-347.

[124] Kho YK, Teoh WY, Iwase A, Mädler L, Kudo A, Amarl R. Flame Preparation of Visible-Light-Responsive $\mathrm{BiVO}_{4}$ Oxygen Evolution Photocatalysts with Subsequent Activation via Aqueous Route. ACS Appl Mater Interfaces 2011;3:1997-2004.

[125] Boningari T, Koirala R, Smirniotis PG. Low-temperature selective catalytic reduction of NO with $\mathrm{NH}_{3}$ over $\mathrm{V} / \mathrm{ZrO}_{2}$ prepared by flame-assisted spray pyrolysis: Structural and catalytic properties. Appl Catal B-Environ 2012;127:255-264.

[126] Hoj M, Kessler T, Beato P, Jensen AD, Grunwaldt JD. Structure, activity and kinetics of supported molybdenum oxide and mixed molybdenum-vanadium oxide catalysts prepared by flame spray pyrolysis for propane OHD. Appl Catal A-Gen 2014;472:29-38.

[127] Rao PM, Cai LL, Liu C, Cho IS, Lee CH, Weisse JM, Yang PD, Zheng XL. Simultaneously Efficient Light Absorption and Charge Separation in $\mathrm{WO}_{3} / \mathrm{BiVO}_{4}$ Core/Shell Nanowire Photoanode for Photoelectrochemical Water Oxidation. Nano Lett 2014;14:1099-1105.

[128] Wang L, Teleki A, Pratsinis SE, Gouma PI. Ferroelectric $\mathrm{WO}_{3}$ nanoparticles for acetone selective 
detection. Chem Mater 2008;20:4794-4796.

[129] Wagloehner S, Nitzer-Noski M, Kureti S. Oxidation of soot on manganese oxide catalysts. Chem Eng J 2015;259:492-504.

[130] van Vegten N, Baidya T, Krumeich F, Kleist W, Baiker A. Flame-made $\mathrm{MgAl}_{2-\mathrm{x}} \mathrm{M}_{\mathrm{x}} \mathrm{O}_{4}(\mathrm{M}=\mathrm{Mn}, \mathrm{Fe}, \mathrm{Co})$ mixed oxides: Structural properties and catalytic behavior in methane combustion. Appl Catal B-Environ 2010;97:398-406.

[131] Choi SH, Kang YC. Characteristics of $\mathrm{ZnMn}_{2} \mathrm{O}_{4}$ Nanopowders Prepared by Flame Spray Pyrolysis for Use as Anode Material in Lithium Ion Batteries. Int J Electrochem Sci 2013;8:6281-6290.

[132] Tamaekong N, Samerjai T, Liewhiran C, Wisitsoraat A, Phanichphant S. The effect of Mn on flame spray pyrolysis-made $\mathrm{ZnO}$ nanoparticles for flammable gases detection. J Nanosci Nanotechnol 2014;14:7860-7864.

[133] Kriegel R, Tpfer J, Preuss N, Grimm S, Ber J. Flame pyrolysis: A preparation route for ultrafine powders of metastable $\beta-\mathrm{SrMnO} 3$ and $\mathrm{NiMn}_{2} \mathrm{O}_{4}$. J Mater Sci Lett 1994;13:1111-1113.

[134] Lu Y, Eyssler A, Otal EH, Matam SK, Brunko O, Weidenkaff A, Ferri D. Influence of the synthesis method on the structure of Pd-substituted perovskite catalysts for methane oxidation. Catal Today 2013;208:42-47.

[135] Buchneva O, Rossetti I, Oliva C, Scavini M, Cappelli S, Sironi B, Allieta M, Kryukov A, Forni L. Effective Ag doping and resistance to sulfur poisoning of La-Mn perovskites for the catalytic flameless combustion of methane. J Mater Chem 2010;20:10021-10031.

[136] Grimm S, Schultz M, Barth S, Muller R. Flame pyrolysis- a preparation route for ultrafine pure gamma- $\mathrm{Fe}_{2} \mathrm{O}_{3}$ powders and the control of their particle size and properties. J Mater Sci 1997;32:1083-1092.

[137] Duret A, Grätzel M. Visible light-induced water oxidation on mesoscopic $\gamma$-Fe2O3 films made by ultrasonic spray pyrolysis. J Phys Chem B 2005;109:17184-17191

[138] Strobel R, Pratsinis SE. Direct synthesis of maghemite, magnetite and wustite nanoparticles by flame spray pyrolysis. Adv Powder Technol 2009;20:190-194.

[139] Rao PM, Zheng XL. Unique Magnetic Properties of Single Crystal $\gamma$-Fe2O3 Nanowires Synthesized by Flame Vapor Deposition. Nano Lett 2011;11:2390-2395.

[140] Li YF, Hu YJ, Huo JC, Jiang H, Li CZ, Huang GJ. Stable Core Shell $\mathrm{Co}_{3} \mathrm{Fe}_{7}-\mathrm{CoFe}_{2} \mathrm{O}_{4}$ Nanoparticles Synthesized via Flame Spray Pyrolysis Approach. Ind Eng Chem Res 2012;51:11157-11162.

[141] Dosev D, Nichkova M, Dumas RK, Gee SJ, Hammock BD, Liu M, Kennedy IM. Magnetic/luminescent core/shell particles synthesized by spray pyrolysis and their application in immunoassays with internal standard. Nanotechnology 2007;18:055102.

[142] Kim T, Guo B. Zn-doped gamma- $\mathrm{Fe}_{2} \mathrm{O}_{3}$ sensors for flammable gas detection: Effect of annealing on sensitivity and stability. J Ind Eng Chem 2011;17:158-164.

[143] Barkley TK, Vastano JE, Applegate JR, Bakrania SD. Combustion Synthesis of Fe-Incorporated SnO2 Nanoparticles Using Organometallic Precursor Combination. Adv Mater Sci Eng 2012;685754:1-8.

[144] Channei D, Inceesungvorn B, Wetchakun N, Phanichphant S, Nakaruk A, Koshy P, Sorrell CC. Photocatalytic activity under visible light of $\mathrm{Fe}$-doped $\mathrm{CeO}_{2}$ nanoparticles synthesized by flame spray pyrolysis. Ceram Int 2012;39:3129-3134.

[145] Gan JA, Berndt CC. ]Design and manufacture of Nd-Fe-B thick coatings by the thermal spray process. Surf Coat Technol 2011;205:4697-4704. 
[146] Chiarello GL, Rossetti I, Forni L. Flame-spray pyrolysis preparation of perovskites for methane catalytic combustion. J Catal 2005;236:251-261.

[147] Chiarello GL, Rossetti I, Forni L, Lopinto P, Mighavacca G. Solvent nature effect in preparation of perovskites by flame-pyrolysis-1. Carboxylic acids. Appl Catal B-Environ 2007;72:218-226.

[148] Chiarello GL, Rossetti I, Forni L, Lopinto P, Mighavacca G. Solvent nature effect in preparation of perovskites by flame pyrolysis - 2. Alcohols and alcohols plus propionic acid mixtures. Appl Catal B-Environ 2007;72:227-232.

[149] Chiarello GL, Grunwaldt JD, Ferri D, Krumeich R, Oliva C, Forni L, Baiker A. Flame-synthesized $\mathrm{LaCoO}_{3}$-supported Pd 1. Structure, thermal stability and reducibility. J Catal 2007;252:127-136.

[150] Chiarello GL, Ferri D, Grunwaldt JD, Forni L, Baiker A. Flame-synthesized LaCoO $\mathrm{L}_{3}$ supported Pd 2. Catalytic behavior in the reduction of $\mathrm{NO}$ by $\mathrm{H}_{2}$ under lean conditions. J Catal 2007;252:137-147.

[151] Buchneva O, Rossetti I, Biffi C, Allieta M, Kryukov A, Lebedeva N. La-Ag-Co perovskites for the catalytic flameless combustion of methane. Appl Catal B-Gen 2009;370:24-33.

[152] Teoh WY, Setiawan R, Mädler L, Grunwaldt JD, Amal R, Pratsinis, SE. Ru-doped Cobalt-Zirconia nanocomposites by flame synthesis: Physicochemical and catalytic properties. Chem Mater 2008;20:4069-4079.

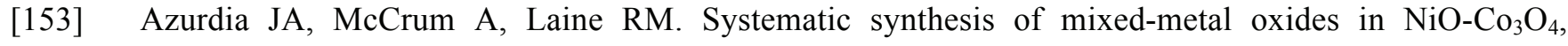
$\mathrm{NiO}-\mathrm{MoO}_{3}$, and NiO-CuO systems via liquid-feed flame spray pyrolysis. J Mater Chem 2008;18:3249-3258.

[154] Martinez U, Asazawa K, Halevi B, Falase A, Kiefer B, Serov A, Padilla M, Olson T, Datye A, Tanaka H, Atanassov P. Aerosol-derived Nil-xZnx electrocatalysts for direct hydrazine fuel cells. Phys Chem Chem Phys 2012;14:5512-5517.

[155] Athanassiou EK, Grass RN, Osterwalder N, Stark, WJ. Preparation of homogeneous, bulk nanocrystalline $\mathrm{Ni} / \mathrm{Mo}$ alloys with tripled vickers hardness using flame-made metal nanoparticles. Chem Mater 2007;19:4847-4854.

[156] Arji R, Dwivedi DK, Gupta SR. Some studies on slurry erosion of flame sprayed Ni-Cr-Si-B coating. Ind Lubr Tribol 2009;61:4-10.

[157] Chiang CY, Aroh K, Franson N, Satsangi VR, Dass S, Ehrman S. Copper oxide nanoparticle made by flame spray pyrolysis for photoelectrochemical water splitting - Part II. Photoelectrochemical study. Int J Hydrog Energy 2011;36:15519-15526.

[158] Rao PM, Zheng XL. Rapid Catalyst-Free Flame Synthesis of Dense, Aligned alpha- $\mathrm{Fe}_{2} \mathrm{O}_{3}$ Nanoflake and $\mathrm{CuO}$ Nanoneedle Arrays. Nano Lett 2009;9:3001-3006.

[159] Cai LL, Rao PM, Feng YZ, Zheng XL. Flame synthesis of 1-D complex metal oxide nanomaterials. Proc Combust Inst 2013;34:2229-2236.

[160] Peng YZ, Cho IS, Rao PM, Cai LL, Zheng XL. Sol-Flame Synthesis: A General Strategy To Decorate Nanowires with Metal Oxide/Noble Metal Nanoparticles. Nano Lett 2013;13:855-860.

[161] Dressick WJ, Soto CM, Fontana J, Baker CC, Myers JD, Frantz JA, Kim W. Preparation and Layer-by-Layer Solution Deposition of $\mathrm{Cu}(\mathrm{In}, \mathrm{Ga}) \mathrm{O}_{2}$ Nanoparticles with Conversion to $\mathrm{Cu}(\mathrm{In}, \mathrm{Ga}) \mathrm{S}_{2}$ Films. PLOS One 2014;9:e100203.

[162] Zhang RD, Teoh WY, Amal R, Chen BH, Kaliaguine S. Catalytic reduction of NO by CO over $\mathrm{Cu} / \mathrm{Ce}_{\mathrm{x}} \mathrm{Zr}_{1-\mathrm{x}} \mathrm{O}_{2}$ prepared by flame synthesis. J Catal 2010;272:210-219.

[163] Kydd R, Teoh WY, Wong K, Wang Y, Scott J, Zeng QH, Yu AB, Zou J, Amal R. Flame-Synthesized 
Ceria-Supported Copper Dimers for Preferential Oxidation of CO. Adv Funct Mater 2009;19:369-377.

[164] Kydd R, Ferri D, Hug P, Scott J, Teoh WY, Amal R. Temperature-induced evolution of reaction sites and mechanisms during preferential oxidation of CO. J Catal 2011;277:64-71.

[165] Huber F, Meland H, Ronning M, Venvik H, Holmen A. Comparison of Cu-Ce-Zr and Cu-Zn-Al mixed oxide catalysts for water-gas shift. Top Catal 2007;45:101-104.

[166] Choi SH, Kang YC. One-pot facile synthesis of Janus-structured $\mathrm{SnO}_{2}-\mathrm{CuO}$ composite nanorods and their application as anode materials in Li-ion batteries. Nanoscale 2013;5:4662-4668.

[167] Widiyandari H, Purwanto A, Balgis R, Ogi T, Okuyama $\mathrm{K} . \mathrm{CuO} / \mathrm{WO}_{3}$ and $\mathrm{Pt} / \mathrm{WO}_{3}$ nanocatalysts for efficient pollutant degradation using visible light irradiation. Chem Eng J 2012;180:323-329.

[168] Lee J, Gouma PI. Flame-spray-processed $\mathrm{CuO}-\mathrm{WO}_{3}$ nanopowders as photocatalysts. J Am Ceram Soc 2014;97:3719-3720.

[169] Height MJ, Pratsinis SE, Mekasuwandumrong O, Praserthdam P. Ag-ZnO catalysts for UV-photodegradation of methylene blue. Appl Catal B-Environ 2006;63:305-312.

[170] Pawinrat P, Mekasuwandumrong O, Panpranot J. Synthesis of Au-ZnO and Pt-ZnO nanocomposites by one-step flame spray pyrolysis and its application for photocatalytic degradation of dyes. Catal Commun 2009;10:1380-1385.

[171] Liewhiran C, PhanichphantS. Doctor-bladed thick films of flame-made $\mathrm{Pd} / \mathrm{ZnO}$ nanoparticles for ethanol sensing. Curr Appl Phys 2008;8:336-339.

[172] Kruefu V, Liewhiran C, Wisitsoraat A, Phanichphant S. Selectivity of flame-spray-made Nb/ZnO thick films towards $\mathrm{NO}_{2}$ gas. Sens Actuator B-Chem 2011;156:360-367.

[173] Kilian D, Polster S, Vogeler I, Jank MPM, Frey L, Peukert W. Pulsed direct flame deposition and thermal annealing of transparent amorphous Indium Zinc oxide films as active layers in field effect transistors. ACS Appl Mater Interfaces 2014;6:12245-12251.

[174] Brehm JU, Winterer M, Hahn H. Synthesis and local structure of doped nanocrystalline Zinc oxides. J Appl Phys 2006;100:064311.

[175] Sigaev VN, Golubev NV, Ignateva ES, Paleari A, Lorenzi R. Light-emitting Ga-oxide nanocrystals in glass: a new paradigm for low-cost and robust UV-to-visible solar-blind converters and UV emitterst. Nanoscale 2014;6:1763-1774.

[176] Ifeacho P, Wiggers $\mathrm{H}$, Schulz C, Schneider L, Bacher $\mathrm{G} . \mathrm{Ga}_{2} \mathrm{O}_{3}$ nanoparticles synthesized in a low-pressure flame reactor. J Electrochem Soc 2008;10:121-127.

[177] Simanzhenkov V, Ifeacho P, Wiggers H, Knipping J, Roth P. Synthesis of germanium oxide nanoparticles in low-pressure premixed flames. J Nanosci Nanotechnol 2004;4:157-161.

[178] Kim KH, Park JK, Kim CH, Park HD, Chang H, Choi SY. Synthesis of SrTiO3:Pr,Al by ultrasonic spray pyrolysis. Ceram Int 2002;28:29-36.

[179] Kang YC, Seo DJ, Park SB, Park HD. Direct synthesis of strontium titanate phosphor particles with high luminescence by flame spray pyrolysis. Mater Res Bull 2002;37:263-269.

[180] Kang YC, Sohn JR, Yoon HS, Jung KY, Park HD.Improved photoluminescence of $\mathrm{Sr}_{5}(\mathrm{PO} 4)_{3} \mathrm{Cl}: \mathrm{Eu}^{2+}$ phosphor particles prepared by flame spray pyrolysis. J Electrochem Soc 2003;150:H38-H42.

[181] Guo B, Harvey A, Risbud SH, Kennedy IM. The formation of cubic and monoclinic $\mathrm{Y}_{2} \mathrm{O}_{3}$ nanoparticles in a gas-phase flame process. Philos Mag Lett 2006;86:457-467.

[182] Dosev D, Guo B, Kennedy IM. Photoluminescence of $\mathrm{Eu}^{3+}: \mathrm{Y}_{2} \mathrm{O}_{3}$ as an indication of crystal structure and 
particle size in nanoparticles synthesized by flame spray pyrolysis. J Aerosol Sci 2006;37:402-412.

[183] Camenzind A, Strobel R, Pratsinis SE. Cubic or monoclinic $\mathrm{Y}_{2} \mathrm{O}_{3}: \mathrm{Eu}^{3+}$ nanoparticles by one step flame spray pyrolysis. Chem Phys Lett 2005;415:193-197.

[184] Lee JS, Kim SJ, Kim TK, Singh RK, Ranade MB. Luminescent properties of $\mathrm{Y}_{2} \mathrm{O}_{3}: \mathrm{Eu}^{3+}$ nanophosphor prepared from urea added precursor using flame spray pyrolysis. J Mater Res 2009;24:2584-2588.

[185] Qin X, Ju YG, Bernhard S, Yao N. Flame synthesis of $\mathrm{Y}_{2} \mathrm{O}_{3}$ :Eu nanophosphors using ethanol as precursor solvents. J Mater Res 2005;20:2960-2968.

[186] Purwanto A, Lenggoro IW, Chang HW, Okuyama K. Preparation of submicron- and nanometer-sized particles of $\mathrm{Y}_{2} \mathrm{O}_{3}: \mathrm{Eu}^{3+}$ by flame spray pyrolysis using ultrasonic and two-fluid atomizers. J Chem Eng Japan 2006;39:68-76.

[187] Kubrin R, Tricoli A, Camenzind A, Pratsinis SE, Bauhofer W. Flame aerosol deposition of $\mathrm{Y}_{2} \mathrm{O}_{3}: \mathrm{Eu}$ nanophosphor screens and their photoluminescent performance. Nanotechnol 2010;21:225603.

[188] Tanner PA, Wong KL. Synthesis and spectroscopy of lanthanide ion-doped $\mathrm{Y}_{2} \mathrm{O}_{3}$. J Phys Chem B 2004;108:136-142.

[189] Hasegawa $\mathrm{H}$, Ueda $\mathrm{T}$, Yokomori $\mathrm{T}$. $\mathrm{Y}_{2} \mathrm{Si}_{2} \mathrm{O}_{7}: \mathrm{Eu} / \mathrm{SiO}_{2}$ core shell phosphor particles prepared by flame spray pyrolysis. Prog Combust Inst 2013;34:2155-2162.

[190] Sotiriou GA, Schneider M, Pratsinis SE. Color-Tunable Nanophosphors by Codoping Flame- $\mathrm{Made}_{2} \mathrm{Y}_{2} \mathrm{O}_{3}$ with Tb and Eu. J Phys Chem C 2011;115:1084-1089.

[191] Sotiriou GA, Schneider M, Pratsinis SE. Green, Silica-Coated Monoclinic $\mathrm{Y}_{2} \mathrm{O}_{3}: \mathrm{Tb}^{3+}$ Nanophosphors: Flame Synthesis and Characterization. J Phys Chem C 2012;116:4493-4499.

[192] Lee JS, Kumar P, Gupta S, Oh MH, Ranade MB, Singh RK. Enhanced Luminescence Properties of YAG:Ce ${ }^{3+}$ Nanophosphor Prepared by Flame Spray Pyrolysis. J Electrochem Soc 2010;157:K25-K29.

[193] Kong WJ, Shan J, Ju, YG. Flame synthesis and effects of host materials on $\mathrm{Yb}^{3+} / \mathrm{Er}^{3+}$ co-doped upconversion nanophosphors. Mater Lett 2010;64:688-691.

[194] Yuan FL, Chen CH, Kelder EM, Schoonman J. Preparation of zirconia and yttria-stabilized zirconia (YSZ) fine powders by flame-assisted ultrasonic spray pyrolysis (FAUSP). Solid State Ion 1998;109:119-123

[195] Geier M, Parker T. Electrospray flame synthesis of Yttria-stabilized Zirconia nanoparticles. Ind Eng Chem Res 2013;52:16842-16850.

[196] Jossen R, Mueller R, Pratsinis SE, Watson M, Akhtar MK. Morphology and composition of spray-flame-made Yttria-stabilized Zirconia nanoparticles. Nanotechnol 2005;16:S609-S617.

[197] Heel A, Vital A, Holtappels P, Graule T. Flame spray synthesis and characterisation of stabilised $\mathrm{ZrO}_{2}$ and $\mathrm{CeO}_{2}$ electrolyte nanopowders for SOFC applications at intermediate temperatures. J Electroceram 2009;22:40-46.

[198] Eggersdorfer ML, Grohn AJ, Sorensen CM, McMurry PH, Pratsinis SE. Mass-mobility characterization of flame-made $\mathrm{ZrO}_{2}$ aerosols: Primary particle diameter and extent of aggregation. J Colloid Interface Sci 2012;387:12-23.

[199] Mueller R, Jossen R, Kammler HK, Pratsinis SE. Growth of zirconia particles made by flame spray pyrolysis. AIChE J 2004;50:3085-3094.

[200] Torabmostaedi H, Zhang T, Foot P, Dembele S, Fernandez C. Process control for the synthesis of ZrO2 nanoparticles using FSP at high production rate. Powder Technol 2013;246:419-433.

[201] Jossen R, Heine MC, Pratsinis SE, Akhtar MK. Thermal stability of flame-made zirconia-based mixed 
oxides. Chem Vap Deposition 2006;12:614-619.

[202] Stark WJ, Maciejewski M, Mädler L, Pratsinis SE, Baiker, A. Flame-made nanocrystalline ceria/zirconia: structural properties and dynamic oxygen exchange capacity. J Catal 2003;220:35-43.

[203] Strobel R, Krumeich F, Pratsinis SE, Baiker A. Flame-derived $\mathrm{Pt} / \mathrm{Ba} / \mathrm{Ce}_{\mathrm{x}} \mathrm{Zr}_{1-\mathrm{x}} \mathrm{O} 2$ : Influence of support on thermal deterioration and behavior as NOx storage-reduction catalysts. J Catal 2006;243:229-238.

[204] Hotz N, Stutz MJ, Loher S, Stark WJ, Poulikakos D. Syngas production from butane using a flame-made $\mathrm{Rh} / \mathrm{Ce}_{0.5} \mathrm{Zr}_{0.5} \mathrm{O}_{2}$ catalyst. Appl Catal B-Environ 2007;37:336-344.

[205] Santis-Alvarez AJ, Buchel R, Hild N, Stark WJ, Poulikakos D. Comparison of flame-made Rhodium on $\mathrm{Al}_{2} \mathrm{O}_{3}$ or $\mathrm{Ce}_{0.5} \mathrm{Zr}_{0.5} \mathrm{O}_{2}$ supports for the partial oxidation of methane. Appl Catal A-Gen 2014;469:275-283.

[206] Samerjai T, Tamaekong N, Wetchakun K, Kruefu V, Liewhiran C, Siriwong C, Wisitsoraat A, Phanichphat S. Flame-spray-made metal-loaded semiconducting metal oxides thick films for flammable gas sensing. Sens Actuator B-Chem 2012;171:43-61.

[207] Merchan-Merchan W, Saveliev AV, Kennedy LA. Flame synthesis of molybdenum oxide whiskers. Chem Phys Lett 2006;422:72-77.

[208] Merchan-MerchanW, Saveliev AV, Desai M. Volumetric flame synthesis of well-defined molybdenum oxide nanocrystals. Nanotechnology 2009; 20: 475601.

[209] Cai LL, Rao PM, Zheng XL. Morphology-controlled flame synthesis of single, branched, and flower-like alpha- $\mathrm{MoO}_{3}$ nanobelt arrays. Nano Lett. 2011;11:872-877.

[210] Høj M, Pham DK, Brorson M, Mädler L, Jensen AD, Grunwaldt JD. Two-nozzle flame spray pyrolysis (FSP) synthesis of $\mathrm{CoMo} / \mathrm{Al}_{2} \mathrm{O}_{3}$ hydrotreating catalysts. Catal Lett 2013;143:386-394.

[211] Schuh K, Kleist W, Høj M, Trouillet V, Jensen AD, Grunwaldt JD. One-step synthesis of bismuth molybdate catalysts via flame spray pyrolysis for the selective oxidation of propylene to acrolein. Chem Commun 2014;50:15404-15406.

[212] Liewhiran C, Tamaekong N, Wisitsoraat A, Phanichphant S. $\mathrm{H}_{2}$ Sensing Response of Flame-Spray-Made $\mathrm{Ru} / \mathrm{SnO}_{2}$ Thick Films Fabricated from Spin-Coated Nanoparticles. Sensors 2009;9:8996-9010.

[213] Sahm T, Rong W, Barsan N, Mädler L, Weimar U. Sensing of $\mathrm{CH}_{4}, \mathrm{CO}$ and ethanol with in situ nanoparticle aerosol-fabricated multilayer sensors. Sensor Actuat B-Chem. 2007;127:63-68.

[214] Sahm T, Rong W, Bârsan N, Mädler L, Friedlander SK, Weimar U. Formation of multilayer films for gas sensing by in situ thermophoretic deposition of nanoparticles from aerosol phase. J Mater Res 2007;22(04):850-857.

[215] Großmann K, Kovacs KE, Pham DK, Mädler L, Barsan N, Weimar U. Enhancing performance of FSP $\mathrm{SnO} 2$-based gas sensors through Sb-doping and Pd-functionalization. Sensor Actuat B-Chem. 2011; 158: 388-392.

[216] Kemmler JA, Pokhrel S, Birkenstock J, Schowalter M, Rosenauer A, Barsan N, Weimar U, Mädler L. Quenched, nanocrystalline $\mathrm{In}_{4} \mathrm{Sn}_{3} \mathrm{O}_{12}$ high temperature phase for gas sensing applications. Sensor Actuat B-Chem. 2012; 161: 740-747.

[217] Katusic S, Albers P, Kern R, Petrat FM, Sastrawan R, Hore S, Hinsch A, Gutsch A. Production and characterization of ITO-Pt semiconductor powder containing nanoscale noble metal particles catalytically active in dye-sensitized solar cells. Sol Energy Mater Sol Cells 2006;90:1983-1999.

[218] Ifeacho P, Huelser T, Wiggers H, Schulz C, Roth P. Synthesis of $\mathrm{SnO}_{2-x}$ nanoparticles tuned between 0 
$<=\mathrm{x}<=1$ in a premixed low pressure $\mathrm{H}_{2} / \mathrm{O}_{2} / \mathrm{Ar}$ flame. Proc Combust Inst 2007;31:1805-1812.

[219] Kemmler JA, Pokhrel S, Mädler L, Weimar U, Barsan N. Flame spray pyrolysis for sensing at the nanoscale. Nanotechnology 2013;24:442001

[220] Keskinen H, Tricoli A, Marjamaki M, Makela JM, Pratsinis SE. Size-selected agglomerates of SnO2 nanoparticles as gas sensors. J Appl Phys 2009;106:084316.

[221] Sahm T, Mädler L, Gurlo A, Barsan N, Pratsinis SE, Weimar U. Flame spray synthesis of tin dioxide nanoparticles for gas sensing. Sensor Actuat B-Chem. 2004;98:148-153.

[222] Mädler L, Roessler A, Pratsinis SE, Sahm T, Gurlo A, Barsan N, Weimar U. Direct formation of highly porous gas-sensing films by in situ thermophoretic deposition of flame-made $\mathrm{Pt} / \mathrm{SnO} 2$ nanoparticles. Sensor Actuat B-Chem. 2006;114:283-295.

[223] Mädler L, Sahm T, Gurlo A, Grunwaldt JD, Barsan N, Weimar U, Pratsinis SE. Sensing low concentrations of $\mathrm{CO}$ using flame-spray-made $\mathrm{Pt} / \mathrm{SnO}_{2}$ nanoparticles. J Nanopart Res 2006;8:783-796.

[224] Kühne S, Graf M, Tricoli A, Mayer F, Pratsinis SE, Hierlemann A. Wafer-level flame-spray-pyrolysis deposition of gas-sensitive layers on microsensors. J Micromech Microeng 2008;18:035040.

[225] Symalla MO, Drochner A, Vogel H, Buchel R, Pratsinis SE, Baiker A. Structure and NOx storage behaviour of flame-made $\mathrm{BaCO}_{3}$ and $\mathrm{Pt} / \mathrm{BaCO}_{3}$ nanoparticles. Appl Catal B-Environ 2009;89:41-48.

[226] Buchel R, Strobel R, Baiker A, Pratsinis SE. Effect of the Proximity of Pt to Ce or $\mathrm{Ba}$ in $\mathrm{Pt} / \mathrm{Ba} / \mathrm{CeO}_{2}$ Catalysts on NOx Storage-Reduction Performance. Top Catal 2009;52:1709-1712.

[227] He F, Yang PP, Wang D, Li CX, Niu N, Gai SL, Zhang ML. Preparation and Up-Conversion Luminescence of Hollow $\mathrm{La}_{2} \mathrm{O}_{3}: \mathrm{Ln}(\mathrm{Ln}=\mathrm{Yb} / \mathrm{Er}, \mathrm{Yb} / \mathrm{Ho})$ Microspheres. Langmuir 2011;27:5616-5623.

[228] Grass RN, Albrecht TF, Krumeich F, Stark WJ. Large-scale preparation of ceria/bismuth metal-matrix nano-composites with a hardness comparable to steel. J Mater Chem 2007;17:1485-1490.

[229] Seo DJ, Ryu KO, Park SB, Kim KY, Song RH. Synthesis and properties of $\mathrm{Ce}_{1-\mathrm{x}} \mathrm{Gd}_{\mathrm{x}} \mathrm{O}_{2-\mathrm{x} / 2}$ solid solution prepared by flame spray pyrolysis. Mater Res Bull 2006;41:359-366.

[230] Im JM, You HJ, Yoon YS, Shin DW. Synthesis of nano-sized gadolinia doped ceria powder by aerosol flame deposition. J Eur Ceram Soc 2007;27:3671-3675.

[231] Iwako Y, Akimoto Y, Omiya M, Ueda T, Yokomori T. Photoluminescence of cubic and monoclinic $\mathrm{Gd}_{2} \mathrm{O}_{3}$ :Eu phosphors prepared by flame spray pyrolysis. J Lumines 2010;130:1470-1474.

[232] Xu F, Tse SD, Al-Sharab JF, Kear BH. Flame synthesis of aligned tungsten oxide nanowires. Appl Phys Lett 2006;88:243115.

[233] Xu F, Liu X, Tse SD, Cosandey F, Kear BH. Flame synthesis of zinc oxide nanowires. Chem Phys Lett 2007;449(1):175-181.

[234] Pokhrel S, Birkenstock J, Schowalter M, Rosenauer A, Mädler L. Growth of ultrafine single crystalline WO3 nanoparticles using flame spray pyrolysis. Cryst Growth Des 2010;10:632-639.

[235] Rao PM, Zheng XL. Flame synthesis of tungsten oxide nanostructures on diverse substrates. Proc Combust Inst 2011;33:1891-1898.

[236] Gupta A, Ifeacho P, Schulz C, Wiggers H. Synthesis of tailored WO3 and WOx $(2.9<\mathrm{x}<3)$ nanoparticles by adjusting the combustion conditions in a $\mathrm{H}_{2} / \mathrm{O}_{2} / \mathrm{Ar}$ premixed flame reactor. Proc Combust Inst 2011;33:1883-1890.

[237] Hammond C, Straus J, Righettoni M, Pratsinis SE, Hermans I. Nanoparticulate Tungsten Oxide for Catalytic Epoxidations. ACS Catal 2013;3:321-327. 
[238] Qiu YF, Yang ML, Fan HB, Zuo YZ, Shao YY, Xu YJ, Yang XX, Yang SH. Nanowires of alpha- and beta-Bi2O3: phase-selective synthesis and application in photocatalysis. Crystengcomm 2011;13:1843-1850.

[239] Rudin T, Wegner K, Pratsinis SE. Uniform nanoparticles by flame-assisted spray pyrolysis (FASP) of low cost precursors. J Nanopart Res 2011;13:2715-2725.

[240] Jossen R, Pratsinis SE, Stark WJ, Mädler L. Criteria for flame-spray synthesis of hollow, shell-like, or inhomogeneous oxides. J Am Ceram Soc 2005;88:1388-1393.

[241] Strobel R, Pratsinis SE. Effect of solvent composition on oxide morphology during flame spray pyrolysis of metal nitrates. Phys. Chem Chem Phys 2011;13:9246-9252.

[242] Hinklin TR. Mixed-metal oxide nanopowders for structural and photonic applications. Ph D Thesis, Univ. Michigan, 2006;p1-5.

[243] Glassman I, Davis KA, Brezinsky K. A gas-phase combustion synthesis process for non-oxide ceramics. Proc Combust Inst 1992;24:1877-1882.

[244] Katz JL, Hung CH. Initial studies of electric field effects on ceramic powder formation in flames. Proc Combust Inst 1990;23:1733-1738.

[245] Calcote HF, Felder W. A new gas-phase combustion synthesis process for pure metals, alloys, and ceramics. Proc Combust Inst 1992;24:1869-1876.

[246] Axelbaum RL, Lottes CR, Huertas JI, Rosen LJ. Gas-phase combustion synthesis of aluminum nitride powder. Proc Combust Inst 1996;26:1891-1897.

[247] Dufaux DP, Axelbaum RL. Nanoscale unagglomerated nonoxide particles from a sodium coflow flame Combust Flame 1995;100:350-358.

[248] Zhang Z, Charalampopoulos TT. Controlled combustion synthesis of nanosized iron oxide aggregates. Proc Combust Inst 1996;26:1851-1858.

[249] Chagger HK, Hainsworth D, Patterson PM, Pourkashanian M, Williams A. The formation of SiO2 from hexamethyldisiloxane combustion in counterflow methane-air flames. Proc Combust Inst 1996;26:1859-1865.

[250] Degussa, Aerosil@brochure 2004.

[251] Kuhner G, Voll M. Manufacture of Carbon Black, Chapter 1 in Carbon Black. ed. Donnet JB, Bansal RC, Wang MJ. Marcel Dekker. New York; 1993.

[252] Rosner DE. Flame synthesis of valuable nanoparticles: recent progress/current needs in areas of rate laws, population dynamics, and characterization. Ind Eng Chem Res 2005;44:6045-6055.

[253] Tsantilis S, Pratsinis SE. Soft-and hard-agglomerate aerosols made at high temperatures. Langmuir 2004:20(14):5933-5939.

[254] Linteris GT, Rumminger MD, Babushok VI. Catalytic inhibition of laminar flames by transition metal compounds. Prog Energy Combust Sci 2008:34(3):288-329.

[255] Pratsinis SE, Zhu WH, Vemury S. The role of gas mixing in flame synthesis of titania powders. Powder Technol 1996;86:87-93.

[256] Johannessen T, Pratsinis SE, Livbjerg H. Computational analysis of coagulation and coalescence in the flame synthesis of titania particles. Powder Technol 2001;118:242-250.

[257] Wegner K, Pratsinis SE. Scale-up of nanoparticle synthesis in diffusion flame reactors. Chem Eng Sci 2003;58:4581-4589.

[258] Glumac NG, Chen YJ, Skandan G, Kear B. Scalable High-Rate Production of Non-Agglomerated 
Nanopowders in Low Pressure Flames. Mater Lett 1998;34,148.

[259] Wegner K, Pratsinis SE. Nozzle-quenching process for controlled flame synthesis of titania nanoparticles. AICHE J 2003;49:1667-1675.

[260] Wegner K, Stark WJ, Pratsinis SE. Flame-nozzle synthesis of nanoparticles with closely controlled size, morphology and crystallinity. Mater Lett 2002;55(5):318-321.

[261] Migliorini F, De Iuliis S, Cignoli F, Zizak G. How "flat" is the rich premixed flame produced by your McKenna burner? Combust Flame 2008;153:384-393.

[262] Senser DW, Morse JS, Cundy VA. Rev Sci Instrum 1985;56(6):1279-1284.

[263] Prucker S, Meier W, Stricker W. A flat flame burner as calibration source for combustion research: temperatures and species concentrations of premixed $\mathrm{H}_{2}$ /air flames. Rev Sci Instrum 1994;65(9):2908-2911.

[264] Cheskis S. Prog Energy Combust Sci 1999;25:233-252.

[265] Hartung G, Hult J, Kaminski CF. A flat flame burner for the calibration of laser thermometry techniques. Meas Sci Technol 2006;17:2485-2493.

[266] Schulz C, Kock BF, Hofmann M, Michelsen H, Will S, B. Bougie, R. Suntz, G. Smallwood. Laser-induced incandescence: recent trends and current questions. Appl Phys B 2006;83:333-354.

[267] Bladh H, Johnsson J, Bengtsson PE. On the dependence of the laser-induced incandescence (LII) signal on soot volume fraction for variations in particle size. Appl Phys B 2008;90:109-125.

[268] Axelsson B, Collin R, Bengtsson PE. Laser-induced incandescence for soot particle size and volume fraction measurements using on-line extinction calibration. Appl Phys B,2000;39:3683-3690

[269] Vanderwal RL, Weiland KJ. Laser-induced incandescence - development and characterization towards a measurement of soot-volume fraction. Appl Phys B 1994;59(4):445-452.

[270] Ehrman SH, Friedlander SK, Zachariah MR. Characteristics of $\mathrm{SiO}_{2} / \mathrm{TiO}_{2}$ nanocomposite particles formed in a premixed flat flame. J Aerosol Sci 1998;29:687-706.

[271] Ulrich GD, Milnes BA, Subramanian NS. Particle Growth in Flames. II: Experimental Results for Silica Particles. Combust Sci Technol 1976;14:243-249.

[272] Ulrich GD, Subramanian NS. Particle Growth in Flames III. Coalescence as a Rate-Controlling Process. Combust Sci Technol 1977;17:119-126.

[273] Ulrich GD, Riehl JW. Aggregation and growth of submicron oxide particles in flames. J Colloid Interface Sci. 1982;87:257-265.

[274] Hardesty DR, Weinberg FJ, Electrical control of particulate pollutants from flames. Fourteenth International Symposium on Combustion 1973;14:907-918.

[275] Tsantilis S, Kammler HK, Pratsinis SE. Population balance modeling of flame synthesis of titania nanoparticles. Chem Eng Sci 2002;57:2139-2156.

[276] Kammler HK, Beaucage G, Kohls DJ, Agashe N, Ilavsky J. Monitoring simultaneously the growth of nanoparticles and aggregates by in situ ultra-small-angle x-ray scattering. J Appl Phys 2005;97:054309.

[277] Arabi-Katbi OI, Pratsinis SE, Morrison PW, Megaridis CM. Monitoring the flame synthesis of TiO2 particles by in-situ FTIR spectroscopy and thermophoretic sampling. Combust Flame 2001;124:560-572.

[278] Kammler HK, Pratsinis SE, Morrison Jr. PW, Hemmerling B. Flame temperature measurements during electrically assisted aerosol synthesis of nanoparticles. Combust Flame 2002;128:369-381.

[279] Lindackers D, Strecker MGD, Roth P, Janzen C, Pratsinis SE. Formation and Growth of $\mathrm{SiO}_{2} \mathrm{Particles}$ in Low Pressure $\mathrm{H}_{2} / \mathrm{O}_{2} / \mathrm{Ar}$ Flames Doped with $\mathrm{SiH}_{4}$. Combust Sci Tech 1997;123:287-315. 
[280] Janzen C, Roth P. Formation and characteristics of $\mathrm{Fe}_{2} \mathrm{O}_{3}$ nano-particles in doped low pressure $\mathrm{H}_{2} / \mathrm{O}_{2} / \mathrm{Ar}$ flames. Combust Flame 2001;125:1150-1161.

[281] Hancock RD, Bertagnolli KE, Lucht RP. Nitrogen and hydrogen CARS temperature measurements in a hydrogen/air flame using a near-adiabatic flat-flame burner. Combust Flame 1997;109(3):323-331.

[282] Wooldridge MS, Torek PV, Donovan MT, Hall DL, Miller TA, Palmer TR, Schrock CR. An experimental investigation of gas-phase combustion synthesis of $\mathrm{SiO} 2$ nanoparticles using a multi-element diffusion flame burner. Combust Flame 2002;131:98-109.

[283] McLean WM, Hardesty DR, Pohl JH. Direct observations of devolatilizing pulverized coal particles in a combustion environment. Eighteenth International Symposiumon Combustion (the Combustion Institute), 1980;18: 1239-1248.

[284] Ma JL, Fletcher TH, Webb BW. Thermophoretic sampling of coal-derived soot particles during devolatilization. Energy Fuels 1995;9:802-808.

[285] Yuan Y, Li SQ, Li GD, Wu N, Yao Q. The transition of heterogeneous-homogeneous ignitions of dispersed coal particle streams. Combust Flame 2014;161:2458-2468.

[286] Yuan Y, Li SQ, Yao Q. Dynamic behavior of sodium release from pulverized coal combustion by phase-selective laser-induced breakdown spectroscopy. Proc Combust Inst 2015;35(2):2339-2346.

[287] Miller TA, Bakrania SD, Perez C, Wooldridge MS. A new method for direct preparation of tin dioxide nanocomposite materials. J Mater Res 2005;20:2977-2987.

[288] Bakrania SD, Miller TA, Perez C, Wooldridge MS. Combustion of multiphase reactants for the synthesis of nanocomposite materials. Combust Flame 2007;148(1-2):76-87.

[289] Bakrania SD, Perez C, Wooldridge MS. Methane-assisted combustion synthesis of nanocomposite tin dioxide materials. Proc Combust Inst 2007;31:1797-1804.

[290] Bakrania SD, Wooldridge MS. The Effects of the Location of Au Additives on Combustion-generated SnO2 Nanopowders for CO Gas Sensing. Sensors 2010;10:7002-7017.

[291] Memon NK, Tse SD, Al-Sharab JF, Yamaguchi H, Goncalves AMB, Kear BH, Jaluria Y, Andrei EY, Chhowalla M. Flame synthesis of graphene films in open environments. Carbon 2011;49(15):5064-5070.

[292] Memon NK, Stephen DT, Chhowalla M, Kear BH. Role of substrate, temperature, and hydrogen on the flame synthesis of graphene films. Proc Combust Inst 2013;34(2):2163-2170.

[293] Memon NK, Kear BH, Tse SD. Transition between graphene-film and carbon-nanotube growth on Nickel alloys in open-atmosphere flame synthesis. Chem Phys Lett 2013;570:90-94.

[294] Memon, NK, Xu F, Sun G, Dunham SJ, Kear BH, Tse SD. Flame synthesis of carbon nanotubes and few-layer graphene on metal-oxide spinel powders. Carbon 2013;63:478-486.

[295] Ren YH, Zhang YY, Li SQ, Law CK. Doping mechanism of Vanadia/Titania nanoparticles in flame synthesis by a novel optical spectroscopy technique. Proc Combust Inst 2015;35(2):2283-2289.

[296] Zhang YY, Li SQ, Ren YH, Yao Q, Tse SD. A new diagnostic for volume fraction measurement of metal-oxide nanoparticles in flames using phase-selective laser-induced breakdown spectroscopy. Proc Combust Inst. 2015;35:3681-3688.

[297] Zhang YY, Li SQ, Ren YH, Yao Q, Law CK. Two-dimensional imaging of gas-to-particle transition in flames by laser-induced nanoplasmas. Appl Phys Lett 2014;104:023115.

[298] Ren YH, Li SQ, Zhang YY, Tse SD, Long MB. Absorption-Ablation-Excitation Mechanism of Laser-Cluster Interactions in a Nanoaerosol System. Phys Rev Lett 2015;114:093401. 
[299] Chung SL, Katz JL. The Counterflow Diffusion Flame Burner: A New Tool for the Study of the Nucleation of Refractory Compounds. Combust. Flame 1985;61(3):271-284.

[300] Zachariah MR, Chin D, Semerjian HG, Katz JL. Silica Particle Synthesis in a Counterflow Diffusion Flame Reactor. Combust Flame 1989;78:287-298.

[301] Zachariah MR, Chin D, Semerjian HG, Katz JL. Dynamic light-scattering and angular dissymmetry for the insitu measurement of silicon dioxide particle synthesis in flames. Appl Optics 1989;28(3):530-536.

[302] Zachariah MR, Semerjian HG. Experimental and numerical studies on refractory particle formation in flames. High Temp Sci 1990;28:113-125.

[303] Katz JL, Hung CH. Ultrafine refractory particle formation in counterflow diffusion flames. Combust. Sci. \& Technol. 1992;82:169-183.

[304] Hung $\mathrm{CH}$, Katz JL. Formation of mixed-oxide powders in flames .1. $\mathrm{TiO}_{2}-\mathrm{SiO}_{2}$. J Mater Res 1992; 7:1861-1869.

[305] Hung $\mathrm{CH}$, Miquel PF, Katz JL. Formation of mixed-oxide powders in flames .2. $\mathrm{SiO}_{2}-\mathrm{GeO}_{2}$ and $\mathrm{Al}_{2} \mathrm{O}_{3}-\mathrm{TiO}_{2}$. J Mater Res 1992;7:1870-1875.

[306] Rulison AJ, Miquel PF, Katz JL. Titania and silica powders produced in a counterflow diffusion flame. J Mater Res 1996;11:3083-3089.

[307] Xing Y, Rosner DE, Koylu UO, Tandon P. Morphological evolution of nanoparticles in diffusion flames: Measurements and modeling. AIChE J 1997;43:2641-2649.

[308] Xing YC, Koylu UO, Rosner DE. In situ light-scattering measurements of morphologically evolving flame-synthesized oxide nanoaggregates. Appl Optics 1999;38:2686-2697.

[309] Miller JA, Kee RJ, Westbrook CK. Chemical kinetics and combustion modeling. Annu Rev Phys Chem 1990;41:345-387.

[310] Bergthorson JM, Goodwin DG, Dimotakis PE. Particle streak velocimetry and CH laser-induced fluorescence diagnostics in strained, premixed, methane-air flames. Proc Combust Inst 2005;30:1637-1644.

[311] Tolmachoff ED, Abid AD, Phares DJ, Campbell CS, Wang H. Synthesis of nano-phase $\mathrm{TiO}_{2}$ crystalline films over premixed stagnation flames. Proc Combust Inst 2009;32:1839-1845.

[312] Murayama M, Kojima S, Uchida K. Uniform deposition of diamond films using a flat flame stabilized in the stagnation-point flow. J Appl Phys 1991;69:7924-7926.

[313] Murayama M, Uchida K. Synthesis of uniform diamond films by flat flame combustion of acetylene/hydrogen/oxygen mixtures. Combust Flame 1992;91:239-245.

[314] Meeks E, Kee RJ, Dandy DS, Coltrin ME. Computational simulation of diamond chemical vapor deposition in premixed $\mathrm{C}_{2} \mathrm{H}_{2} / \mathrm{O}_{2} / \mathrm{H}_{2}$ and $\mathrm{CH}_{4} / \mathrm{O}_{2}$-strained flames. Combust Flame 1993;92:144-160.

[315] Bertagnolli KE, Lucht RP. Temperature profile measurements in stagnation-flow, diamond-forming flames using hydrogen cars spectroscopy. Proc Combust Inst 1996;26:1825-1833.

[316] Zhao B, Uchikawa K, McCormickb JR, Ni CY, Chen JG, Wang H. Ultrafine anatase $\mathrm{TiO}_{2}$ nanoparticles produced in premixed ethylene stagnation flame at $1 \mathrm{~atm}$. Proc Combust Inst 2005;30:2569-2576.

[317] Zhao B, Uchikawa K, Wang H. A comparative study of nanoparticles in premixed flames by scanning mobility particle sizer, small angle neutron scattering, and transmission electron microscopy. Proc Combust Inst 2007;31:851-860.

[318] Memarzadeh S, Tolmachoff ED, Phares DJ, Wang H. Properties of nanocrystalline $\mathrm{TiO}_{2}$ synthesized in premixed flames stabilized on a rotating surface. Proc Combust Inst 2011;33:1917-1924. 
[319] Zhao H, Liu XF, Tse SD. Control of nanoparticle size and agglomeration through electric-field-enhanced flame synthesis. J Nanopart Res 2008;10:907-923.

[320] Zhao H, Liu XF, Tse SD. Effects of pressure and precursor loading in the flame synthesis of titania nanoparticles. J Aerosol Sci 2009;40:919-937.

[321] Thimsen E, Biswas P. Nanostructured photoactive films synthesized by a flame aerosol reactor. AIChE J 2007;53:1727-1735.

[322] Thimsen E, Rastgar N, Biswas, P. Nanostructured $\mathrm{TiO}_{2}$ films with controlled morphology synthesized in a single step process: performance of dye-sensitized solar cells and photo water-splitting. J Phys Chem 2008;112:4134-4140.

[323] Zhang YY, Li SQ, Yan W, Yao Q. Nanoparticle transport and deposition in boundary layer of stagnation-point premixed flames. Powder Technol 2012;227:24-34.

[324] Zong YC, Li SQ, Niu F, Yao Q. Direct synthesis of supported palladium catalysts for methane combustion by stagnation swirl flame. Proc Combust Inst 2015;35(2):2249-2257.

[325] Libby PA, Williams FA. Strained premixed laminar flames under nonadiabatic conditions. Combust Sci Technol 1983;31:1-42.

[326] Li SQ, Zhang YY, Wang JJ, Deng SL, Tse SD. Synthesis of titania nanoparticles by premixed stagnation flames with different stabilization techniques. Proc. 6th World Congress Particle Technol., April 26-29, 2010, Nuremberg.

[327] Mueller R, Mädler L, Pratsinis SE. Nanoparticle synthesis at high production rates by flame spray pyrolysis. Chem Eng Sci 2003;58:1969-1976.

[328] Gröhn AJ, Pratsinis SE, Wegner K. Fluid-particle dynamics during combustion spray aerosol synthesis of ZrO2. Chem Eng J 2012;191:491-502.

[329] Gröhn AJ, Pratsinis SE, Sanchez-Ferrer A, Mezzenga R, Wegner K. Scale-up of Nanoparticle Synthesis by Flame Spray Pyrolysis: The High-Temperature Particle Residence Time. Ind Eng Chem Res 2014;53:10734-10742.

[330] Rosebrock CD, Riefler N, Wriedt T, Mädler L, Tse SD. Disruptive burning of precursor/solvent droplets in flame-spray synthesis of nanoparticles. AIChE J 2013:59(12):4553-4566.

[331] Meierhofer F, Hodapp M, Achelis L, Buss1 L, Noriler D, Meier HF, Fritsching U. Investigation of atomization concepts for large-scale flame spray pyrolysis (FSP). Mat-wiss. u. Werkstofftech. 2014;45(8):765-778.

[332] Chen DR, Pui DYH, Kaufman SL. Electrospraying of conducting liquids for monodisperse aerosol generation in the $4 \mathrm{~nm}$ to $1.8 \mu \mathrm{m}$ diameter range. J Aerosol Sci 1995;26:963-977.

[333] Rosell-Llompart J, Fernandez de la Mora J. Generation of monodisperse droplets 0.3 to $4 \mu \mathrm{m}$ in diameter from electrified conejets of highly conducting and viscous liquids. J Aerosol Sci 1994;25:1093-1119.

[334] Ganan-Calvo AM, Davila J, Barrero A. Current and droplet size in the electrospraying of liquids. Scaling laws. J Aerosol Sci 1997;28:249-275.

[335] Hogan CJ Jr., Biswas P. Narrow size distribution nanoparticle production by electrospray processing of ferritin. J Aerosol Sci. 2008;39:432-440.

[336] Almería B, Fahmy TM, Gomez A. A multiplexed electrospray process for single-step synthesis of stabilized polymer particles for drug delivery. J Control Release 2011;154(2):203-210. 
[337] Okuyama K, Lenggoro IW. Preparation of nanoparticles via spray route. Chem Eng Sci 2003;58(3):537-547.

[338] Widiyastuti W, Wang WN, Purwanto A, Lenggoro IW, Okuyama K. A pulse combustion-spray pyrolysis process for the preparation of nano- and submicrometer- sized oxide particles. J Am Ceram Soc 2007;90(12):3779-3785.

[339] Nandiyanto A B D, Okuyama K. Progress in developing spray-drying methods for the production of controlled morphology particles: from the nanometer to submicrometer size ranges. Adv Powder Technol 2011;22(1):1-19.

[340] Biswas P, Wu CY, Zachariah MR, McMillin B, Characterization of iron oxide-silica nanocomposites in flames. 2. Comparison of discrete-sectional model predictions to experimental data. J Mater Res 1997; 12:714-723.

[341] Yang G, Wang Z, Biswas P, Bresser W, Boolchand P, Processing titania based materials in flame aerosol reactors: from dopants to nanocomposites. J Aerosol Sci 1998;29:129-130.

[342] Wang ZM, Yang GX, Biswas P, Bresser W, Boolchand P, Processing of iron-doped titania powders in flame aerosol reactors. Powder Technol 2001;114:197-204.

[343] Worathanakul P, Jiang JK, Biswas P, Kongkachuichay P, Quench-ring assisted flame synthesis of SiO2-TiO2 nanostructured composite. J Nanosci Nanotechnol 2008;8:6253-6259.

[344] Sahu M, Biswas P, Single-step processing of copper-doped titania nanomaterials in a flame aerosol reactor. Nanoscale Res Lett 2011;6(1):1-14.

[345] Sahu M, Park J, Biswas $\mathrm{P}$, In situ charge characterization of $\mathrm{TiO}_{2}$ and $\mathrm{Cu}-\mathrm{TiO}_{2}$ nanoparticles in a flame aerosol reactor. J Nanopart Res 2012;14:1-11.

[346] Strobel R, Krumeich F, Stark W J, Pratsinis SE, Baiker A. Flame spray synthesis of $\mathrm{Pd} / \mathrm{Al}_{2} \mathrm{O}_{3}$ catalysts and their behavior in enantioselective hydrogenation. J Catal 2004;222:307-314.

[347] Huang J, Vegten N. V, Jiang Y, Hunger M, Baiker A. Increasing the BrØsted Acidity of Flame-Derived Silica/Alumina up to Zeolitic Strength. Angew Chem Int Ed.2010;49:7776-7781.

[348] Schaub R, Wahlström E, Rønnau A, Lægsgaard E, Stensgaard I, Besenbacher F. Oxygen-Mediated Diffusion of Oxygen Vacancies on the $\mathrm{TiO}_{2}(110)$ Surf Sci 2003;299:377-379.

[349] Lopez N, Nørskov JK, Janssens TVW, Carlsson A, Puig-Molina A, Clausen BS, Grunwaldt JD. The adhesion and shape of nanosized Au particles in a Au/TiO2 catalyst. J Catal 2004;225:86-94.

[350] Thybo S, Jensen S,Johansen J,Johannessen T, Ole Hansen O, Quaade UJ. Flame spray deposition of porous catalysts on surfaces and in microsystems. J Catal 2004;223:271-277.

[351] Pisduangdaw S, Mekasuwandumrong O, Fujita S, Arai M, Yoshida H, Panpranot J. One step synthesis of $\mathrm{Pt}-\mathrm{Co} / \mathrm{TiO}_{2}$ catalysts by flame spray pyrolysis for the hydrogenation of 3-nitrostyrene. Catal Commun 2015;61:11-15.

[352] Kwak JH, Hu J, Mei D, Yi C, Kim DH, Peden CHF, Allard LF, Szanyi J. Coordinatively Unsaturated $\mathrm{Al} 3+$ Centers as Binding Sites for Active Catalyst Phases of Platinum on $\gamma-\mathrm{Al}_{2} \mathrm{O}_{3}$. Science 2009;325:1670-1673.

[353] Chen X, Liu L, Yu PY, Mao SS. Increasing Solar Absorption for Photocatalysis with Black Hydrogenated Titanium Dioxide Nanocrystals. Science 2011;331:746-750.

[354] Pisduangdaw S, Panpranot J, Methastidsook C, Chaisuk C, Faungnawakij K, Praserthdam P, Mekasuwandumrong $\mathrm{O}$. Characteristics and catalytic properties of $\mathrm{Pt}-\mathrm{Sn} / \mathrm{Al}_{2} \mathrm{O}_{3}$ nanoparticles synthesized by 
one-step flame spray pyrolysis in the dehydrogenation of propane. Appl Catal A-Gen 2009;370:1-6.

[355] Pisduangdaw S, Panpranot J, Chaisuk C, Faungnawakij K, Mekasuwandumrong O. Flame sprayed tri-metallic $\mathrm{Pt}-\mathrm{Sn}-\mathrm{X} / \mathrm{Al}_{2} \mathrm{O}_{3}$ catalysts $(\mathrm{X}=\mathrm{Ce}, \mathrm{Zn}$, and $\mathrm{K})$ for propane dehydration. Catal Commun 2011;12:1161-1165

[356] Kim CH, Qi G, Dahlberg K, Li W.Strontium-Doped Perovskites Rival Platinum Catalysts for Treating NOx in Simulated Diesel Exhaust. Science 2010;327:1624-1627.

[357] Rossetti I, Forni L. Catalytic flameless combustion of methane over perovskites prepared by flame-hydrolysis. Appl Catal B-Environ 2001;33:345-352

[358] Lu Y, Michalow KA, Matam SK, Alexander Winkler A, Maegli AE, Yoon S, Heel A, Weidenkaff A, Ferri D. Methane abatement under stoichiometric conditions on perovskite-supported palladium catalysts prepared by flame spray synthesis. Appl Catal B-Environ 2014;144:631-643

[359] Liu G, Li J, Yang K, Tang W, Liu H, Yang J, Yue R, Chen Y. Effects of cerium incorporation on the catalytic oxidation of benzene over flame-made perovskite $\mathrm{La}_{1-\mathrm{x}} \mathrm{CexMnO}_{3}$ catalysts. Particuology 2015;19:60-68.

[360] Leanza R, Rossett I, Fabbrini L, Oliva C, Forni L. Perovskite catalysts for the catalytic flameless combustion of methane Preparation by flame-hydrolysis and characterization by TPD-TPR-MS and EPR. Appl Catal B-Environ 2000;28:55-64.

[361] Chaisuk C, Boonpitak P, Panpranot J, Mekasuwandumrong O. Effects of Co dopants and flame conditions on the formation of $\mathrm{Co} / \mathrm{ZrO} 2$ nanoparticles by flame spray pyrolysis and their catalytic properties in CO hydrogenation. Catal Commun 2011;12:917-922.

[362] Weidenhof B, Reiser M, K. Stöwe, Maier WF, Kim M, Azurdia J, Gulari E,. Seker E, Barks A, Laine RM. High-throughput screening of nanoparticle catalysts made by flame spray pyrolysis as hydrocarbon/no oxidation catalysts. J Am Chem Soc 2009,131,9207-9219.

[363] Teoh WY. A perspective on the Flame Spray Synthesis of Photocatalyst Nanoparticles. Materials 2013;6:3194-3212.

[364] Tian B, Li C, Gu F, Jiang H, Hu Y, Zhang J. Flame sprayed V-doped TiO2 nanoparticles with enhanced photocatalytic activity under visible light irradiation. Chem Eng J 2009;151:220-227.

[365] Chiarello G L, Selli E, Forni L. Photocatalytic hydrogen production over flame spray pyrolysis-synthesised TiO2 and $\mathrm{Au} / \mathrm{TiO} 2$. Appl Catal B-Environ 2008;84:332-339.

[366] Tiwari V, Jiang J, Sethi V, Biswas P. One-step synthesis of noble metal-titanium dioxide nanocomposites in a flame aerosol reactor. Appl Catal A-Gen 2008;345:241-246.

[367] Jiang Y, Jason S, Rose A. Exploring the relationship between surface structure and photocatalytic activity of flame-made $\mathrm{TiO}_{2}$-based catalysts. Appl Catal B-Environ 2012;126:290-297.

[368] Akurati KK, Vital A, Dellemann JP, Michalow K, Graule D, Ferri D, Baiker A. Flame-made $\mathrm{WO}_{3} / \mathrm{TiO}_{2}$ nanoparticles: Relation between surface acidity, structure and photocatalytic activity. Appl Catal B-Environ 2008; 79(1): 53-62.

[369] Tanaka K, Capule M F V, Hisanaga T. Effect of crystallinity of $\mathrm{TiO}_{2}$ on its photocatalytic action. Chemical Physics Letters. 1991, 187(1): 73-76.

[370] Prabhu S, Nithya A, Mohan S C, Jothivenkatachalam K. Synthesis, surface acidity and photocatalytic activity of WO3/TiO2 nanocomposites-an overview. Materials science forum. 2014, 781:63-78.

[371] Qin X, Yokomori T, Ju Y. Flame synthesis and characterization of rare-earth $\left(\mathrm{Er}^{3+}, \mathrm{Ho}^{3+}\right.$, and $\left.\mathrm{Tm}^{3+}\right)$ 
doped upconversion nanophosphors. Appl Phys Lett 2007; 90: 073104.

[372] Purwanto A, Wang W, Ogi T, Lenggoro IW, Eishi Tanabe E, Okuyama, K. High luminance YAG:Ce nanoparticles fabricated from urea added aqueous precursor by flame process. J Alloy Compd 2008,463:350-357.

[373] Zhao N, Gao MY. Magnetic janus particles prepared by a flame synthetic approach: synthesis, characterizations and properties. Adv Mater 2009;21:184-187.

[374] Zhi MJ, Xiang CC, Li JT, Li M, Wu NQ. Nanostructured carbon-metal oxide composite electrodes for supercapacitors: a review. Nanoscale 2013;5(1):72-88.

[375] Ko YN, Park SB, Choi SH, Kang YC. One-pot synthesis of Manganese oxide-carbon composite microspheres with three dimensional channels for Li-ion batteries. Sci Rep 2014;4:5751.

[376] Guo JC, Liu Q, Wang CS, Zachariah MR. Interdispersed amorphous MnOx-carbon nanocomposites with superior electrochemical performance as lithium-storage material. Adv Funct Mater 2012;22:803-811.

[377] Poizot P, Laruelle S, Grugeon S, Dupont L, Tarascon JM. Nano-sized transition-metal oxides as negative-electrode materials for lithium-ion batteries. Nature 2000;407(6803):496-499.

[378] Gerhardt P, Loffler S, Homann KH. The formation of polyhedral carbon ions in fuel-rich acetylene and benzene flames, Proc Combust Inst 1988;22(1):395-401.

[379] Bachmann M, Wiese W, Homann K.-H. PAH and aromers: precursors of fullerenes and soot, Proc Combust Inst 1996;26:2259-2267.

[380] Howard JB. Fullerene formation in flames, Proc Combust Inst 1992;24:933-946.

[381] Grieco WJ, Lafleur AL, Swallow KC, Richter H, Taghizadeh K, Howard JB. Fullerenes and PAH in low-pressure premixed benzene/oxygen flames, Proc Combust Inst 1998;27:1669-1675.

[382] Richter H, Labrocca AJ, Grieco WJ, Taghizadeh K, Lafleur AL, Howard JB. Generation of higher fullerenes in flames, J Phys Chem B 1997;101:1556-1560.

[383] Hebgen P, Goel A, Howard JB, Rainey LC, Vander Sande JB. Synthesis of fullerenes and fullerenic nanostructures in a low-pressure benzene/oxygen diffusion flame. Proc Combust Inst 2000;28:1397-1404.

[384] Duan HM, Mc Kinnon JT. Nanoclusters produced in flames. J Phys Chem 1994;98-49:12815-12818.

[385] Chowdhury KD, Howard JB, Vander Sande JB. Fullerenic Nanostructures in Flames. J Mater Res 1996; 11(02):341-347.

[386] Baker RTK. Catalytic growth of carbon filaments. Carbon 1989;27(3):315-323.

[387] Oberlin A, Endo M, Koyama T. Filamentous growth of carbon through benzene decomposition, J. Crystal Growth 1976;32(3):335-49.

[388] Dai H, Rinzlev AG, Nikolaev P, Thess A, Colbert D, Smalley RE. Single-wall nanotubes produced by metal-catalyzed disproportionation of carbon monoxide. Chem Phys Lett 1996;260:471-475.

[389] Serp P, Figueiredo JL. Carbon Materials for Catalysis, John Wiley \& Sons; Hoboken 2009:309-372.

[390] Bucky Balls, Fullerenes, and the Future: An Oral History Interview with Professor Richard E. Smalley January 22, 2000

[391] Nikolaev P, Bronikowski MJ, Bradley RK, Rohmund F, Colbert DT, Smith KA. Smalley RE. Gas-phase catalytic growth of single-walled carbon nanotubes from carbon monoxide. Chem Phys Lett 1999;313:91-97.

[392] Vander Wal RL, Hall LJ. Flame synthesis of Fe catalyzed single-walled carbon nanotubes and Ni catalyzed nanofibers: growth mechanisms and consequences. Chem Phys Lett 2001;349:178-184.

[393] Rodriguez NM, A review of catalytically grown carbon nanofibers. J Mater Res 1993;8-12:3233-3250. 
[394] Diener MD, Nichelson N, Alford JM., Synthesis of single-walled carbon nanotubes in flames. J Phys Chem B 2000;104:9615-9620.

[395] Pope CJ, Marr JA, Howard JB, Chemistry of fullerenes C60 and C70 formation in flames. J Phys Chem 1993;97:11001.

[396] Homann K-H., Fullerenes and soot formation - new pathways to large particles in flames. Angew Chem Int Ed Engl 1998;37:2434-2451.

[397] Vander Wal RL, Ticich TM, Curtis VE, Diffusion flame synthesis of single-walled carbon nanotubes. Chem Phys Lett 2000;323:217-223.

[398] Merchan-Merchan W, Saveliev A, Kennedy LA, Fridman A, Formation of carbon nanotubes in counter-flow, oxy-methane diffusion flames without catalysts. Chem Phys Lett 2002;354:20-24.

[399] Unrau CJ, Axelbaum RL, Biswas P, Fraundorf P. Synthesis of single-walled carbon nanotubes in oxy-fuel inverse diffusion flames with online diagnostics. Proc Combust Inst 2007;31(2):1865-1872.

[400] Vander Wal RL, Lee JH, Ferrocene as a precursor reagent for metal-catalyzed carbon nanotubes: competing effects. Combust Flame 2002;130:27-36.

[401] Vander Wal RL, Fe-catalyzed single-walled carbon nanotube synthesis within a flame environment. Combust. Flame 2002;130:37-47.

[402] Vander Wal RL, Ticich TM, Flame and furnace synthesis of single-walled and multi-walled carbon nanotubes and nanofibers. J Phys Chem B 2001;105:10249-10256.

[403] Vander Wal RL, Ticich TM, Comparative flame and furnace synthesis of single-walled carbon nanotubes. Chem Phys Lett 2001;336:24-32.

[404] Height MJ, Howard JB, Tester JW, Synthesis of single-walled carbon nanotubes in a premixed acetylene/oxygen/argon flame, Proceedings of the Third Joint Meeting of the U.S. Section of the Combustion Institute 2003.

[405] Goel A, Hebgen P, Vander Sande JB, Howard JB. Combustion synthesis of fullerenes and fullerenic nanostructures. Carbon 2002;40:177-182.

[406] Height MJ, Howard JB, Tester JW. Flame synthesis of single-walled carbon nanotubes, Proc Combust Inst 2005;30:2537-2543.

[407] Zak A. Synthesis of carbon nanotubes using methane-air premixed flames seeded with ferrocene, M.S. Thesis, Rutgers University, 2008.

[408] Dato A, Radmilovic V, Lee Z, Phillips J, Frenklach M. Substrate-free gas-phase synthesis of graphene sheets. Nano lett 2008;8(7):2012-2016.

[409] Dato A, Frenklach M. Substrate-free microwave synthesis of graphene: experimental conditions and hydrocarbon precursors. New J Phys 2010;12(12):125013.

[410] Minutolo P, Commodo M, Santamaria A, De Falco G, D'Anna A. Characterization of flame-generated 2-D carbon nano-disks. Carbon 2014;68:138-148.

[411] Mostofizadeh A, Li Y, Song B, Huang Y. Synthesis, properties, and applications of low-dimensional carbon-related nanomaterials. J Nanomater 2011;16.

[412] Staver AM, Gubareva NV, Lyamkin AI. The flow in a shock tube in the presence of suspended particles. Fizika Goreniya I Vzryza 1984;20(5).

[413] Greiner NR, Phillips DS, Johnson JD, Volk F. Diamonds in detonation soot. Nature 1988.

[414] Howard W, Huang D, Yuan J, Frenklach M, Spear KE, Koba R, Phelps AW. Synthesis of diamond 
powder in acetylene oxygen plasma. J Appl Phys 1990;68(3):1247-1251.

[415] Hunt AT, Carter WB, Cochran Jr. JK. Combustion chemical vapor deposition: a novel thin-film deposition technique. Appl Phys Lett 1993;63(2):266-268.

[416] Polley TA, Carter WB, Poker DB. Deposition of zinc oxide thin films by combustion CVD. Thin Solid Films 1999;357:132-136.

[417] Liu Y, Zha SW, Liu ML. Novel nanostructured electrodes for solid oxide fuel cells fabricated by combustion chemical vapor deposition (CVD). Adv Mater 2004;16(3):256-260.

[418] Choy KL, Charojrochkul S, Steele BCH. Fabrication of cathode for solid oxide fuel cells using flame-assisted vapour deposition technique. Solid State Ion 1997;96:49-54.

[419] Meng G, Song H, Dong Q, Peng D. Application of Novel Aerosol-Assisted Chemical Vapor Deposition Techniques for SOFC Thin Films. Solid State Ion 2004:175:29-34

[420] Maric R, Roller J, Neagu R. Flame-based technologies and reactive spray deposition technology for low-temperature solid oxide fuel cells: technical and economic aspects. J Thermal Spray Technol 2011;20:696-718.

[421] Maric R, Neagu R, Zhang-Steenwinkel Y, Van Berkel FP, Rietveld B. Reactive Spray Deposition Technology-An one-step deposition technique for Solid Oxide Fuel Cell barrier layers. J Power Sources 2010;195(24):8198-8201.

[422] Maric R, Roller JM, Neagu R, Fatih K, Tuck A. Low Pt thin cathode layer catalyst layer by reactive spray deposition technology. ECS Transactions 2008;12(1):59-63.

[423] Neagu R, Zhang X, Maric R, Roller JM. Characterisation and performance of SOFC components made by Reactive Spray Deposition Technology. ECS Transactions 2009;25(2):2481-2486.

[424] Jain R, Maric R. Synthesis of nano-Pt onto ceria support as catalyst for water-gas shift reaction by Reactive Spray Deposition Technology. Appl Catal A-Gen 2014;475:461-468.

[425] Chen YJ, Wu JM, Lin CS, Jhan GY, Wong MS, Ke SC, Lo HH. Role of carbon in titania as visible-light photocatalyst prepared by flat-flame chemical vapor condensation method. J Vac Sci Technol A 2009;27(4):862-866.

[426] Al-Sharab J, Halim H, Ahmed S, Asefa T, Birnie D, Tse SD, Kear B. Flame synthesis of C-doped $\mathrm{TiO}_{2}$ nanopowders for dye sensitized solar cells. Microsc microanal 2011;17(S2):1694-1695.

[427] Halfer T, Zhang HJ, Mädler L, Rezwan K. Ceramic mask-assisted flame spray pyrolysis for direct and accurate patterning of metal oxide nanoparticles. Adv Eng Mater 2013;15:773-779.

[428] An WJ, Thimsen E, Biswas P. Aerosol-chemical vapor deposition method for synthesis of nanostructured metal oxide thin films with controlled morphology. J Phys Chem Lett 2010;1:249-253.

[429] Batchelor GK, Shen C. Thermophoretic deposition of particles in gas flowing over cold surfaces. J Colloid Interface Sci 1985;107:21-37.

[430] Thomson JJ, Thomson GP. Conduction of Electricity through Gases, Vol. I, Cambridge University Press, 1928, p. 399.

[431] Calcote H. Electrical properties of flames, Proc Combust Inst 1949;3:245-253.

[432] Lawton J, Weinberg FJ. Electrical aspects of combustion. Clarendon Press: 1996.

[433] Brande WT. The Bakerian lecture: on some new electrochemical phenomena. Philos Trans R Soc $1814 ; 104: 51-61$. 
[434] Jaggers HC, von Engel A. The effect of electric fields on the burning velocity of various flames, Combust Flame 1971;16:275-285.

[435] Bowser RJ, Weinberg FJ. The effect of direct electric fields on normal burning velocity, Combust Flame 1972;18:296-300.

[436] Jaggers HC, Boeser RJ, Weinberg FJ. The effect of electric fields on burning velocity, Combust Flame 1972;19:135-136.

[437] Berman CH, Gill RJ, Calcote HF, Xiong TY, Enhanced flame stability using electric fields, GRI Report GRI-92/0518. 1993.

[438] Maupin CL, Harris HH. Electrical perturbation of cellular premixed propane/air flames, Combust Flame 1994;97:435-439.

[439] Kono M, Carleton FB, Jones AR, Weinberg FJ, The effect of nonsteady electric fields on sooting flames, Combust Flame 1989;78:357-364.

[440] Saito M, Sato M, Sawada K, Variation of flame shape and soot emission by applying electric field, J. Electrost 1997;39:305-311.

[441] Saito M, Arai T, Arai M, Control of soot emitted from acetylene diffusion flames by applying an electric field, Combust Flame 1999;119:356-366.

[442] Berman CH, Calcote HF, Gill RJ, Keil DG, Enhanced soot radiation using electric fields, GRI Report GRI-87/0237, 1987.

[443] Xie L, Kishi T, Kono M, Investigation of the effect of electric fields on soot formation and flame structure on diffusion flames, Proc Combust Inst 1992;24:1059-1066.

[444] Berman CH, Gill RJ, Calcote HF, NOx Reduction in Flames Stabilized by an Electric Field, ASME Fossil Fuel Combustion Symposium, PD-Vol. 33, R. Ruiz, Ed., New York: ASME, 1991, pp:71-75.

[445] Sher E, Pinhasi G, Pokryvailo A, Bar-On R. Extinction of pool flames by means of a DC electric field, Combust Flame 1993;94:244-252.

[446] Sher E, Pokryvailo A, Jacobson E., and Mond M. Extinction of flames in a nonuniform electric field, Combust Sci Technol 1993;87:59-67.

[447] Oshisa H, Kimura I, Horisawa H. Control of soot emission of a turbulent diffusion flame by DC or AC Corona Discharges. Combust Flame 1999;116:653-661.

[448] Onda K, Kasuga Y, Kato K, Fujiwara M, Tanimoto M. Electric discharge removal of SO2 and NOx from combustion flue gas by pulsed corona discharge. Energy Conv Manag 1997;38:1377-1387.

[449] Chattock AP. On the velocity and mass of ions in the electric wind in air, Philos Mag 1899;48:401.

[450] Hardesty DR, Weinberg FJ. Electrical control of particulate pollutants from flames, Proc Combust Inst 1973;14:907-918.

[451] Katz JL, Hung C, Untrafine refractory particle formation in counterflow diffusion flames, Combust Sci Technol 1992;82:169-183.

[452] Vemury S, Pratsinis SE. Corona-assisted flame synthesis of ultrafine titania particles. Appl Phys Lett 1995;66(24):3275-3277.

[453] Vemury S, Pratsinis SE, Kibbey L. Electrically controlled flame synthesis of nanophase TiO2, SiO2, and SnO2 powders. J Mater Res 1997;12(04):1031-1042. 
[454] Morrison PW, Raghavan R, Timpone AJ, Artelt CP, Pratsinis, SE. In situ Fourier transform infrared characterization of the effect of electrical fields on the flame synthesis of $\mathrm{TiO}_{2}$ particles. Chem Mat 1997;9(12):2702-2708.

[455] Kammler HK, Pratsinis SE. Electrically-assisted flame aerosol synthesis of fumed silica at high production rates. Chem Eng Process 2000;39(3):219-227.

[456] Fialkov AB. Investigations on ions in flames. Prog Energy Combust Sci 1997;23(5):399-528.

[457] Katzer M, Weber AP, Kasper G. The effects of electrical fields on growth of titania particles formed in a $\mathrm{CH}_{4}-\mathrm{O}_{2}$ diffusion flame. J Aerosol Sci 2001;32(9):1045-1067.

[458] Merchan-Merchan W, Saveliev AV, Kennedy LA. Flame nanotube synthesis in moderate electric fields: From alignment and growth rate effects to structural variations and branching phenomena. Carbon 2006;44(15):3308-3314.

[459] Xu F, Liu X, Tse SD. Synthesis of carbon nanotubes on metal alloy substrates with voltage bias in methane inverse diffusion flames. Carbon 2006;44:570-577.

[460] Xu F, Zhao H, Stephen DT. Carbon nanotube synthesis on catalytic metal alloys in methane/air counterflow diffusion flames. Proc Combust Inst 2007;31(2):1839-1847.

[461] Tolmachoff E, Memarzadeh S, Wang H. Nanoporous titania gas sensing films prepared in a premixed stagnation flame. J Phys Chem C 2011;115:21620-21628.

[462] Nikraz S, Phares DJ, Wang H. Mesoporous titania films prepared by flame stabilized on a rotating surface: application in dye sensitized solar cells. J Phys Chem C 2012;116:5342-5351.

[463] Tricoli A, Righettoni M, Pratsinis SE. Anti-fogging nanofibrous $\mathrm{sio}_{2}$ and nanostructured $\mathrm{sio}_{2}$-tio $\mathrm{films}_{2}$ made by rapid flame deposition and in situ annealing. Langmuir 2009;25:12578-12584.

[464] Tricoli A, Graf M, Mayer F, Kühne S, Hierlemann A and Pratsinis SE. Micropatterning layers by flame aerosol deposition-annealing. Adv Mater 2008;20:3005-3010.

[465] Righettoni M, Tricoli A, Pratsinis SE. Si: $\mathrm{WO}_{3}$ sensors for noninvasive diabetes diagnosis by breath analysis. Sensors 2010:1491-1495.

[466] Liewhiran C, Phanichphant S. Influence of thickness on ethanol sensing characteristics of doctor-bladed thick film from flame-made $\mathrm{ZnO}$ nanoparticles. Sensors 2007;7:185-201.

[467] Liewhiran C, Phanichphant S. Effects of Palladium Loading on the Response of a Thick Film Flame-made ZnO Gas Sensor for Detection of Ethanol Vapor. Sensors 2007;7:1159-1184.

[468] Tamaekong N, Liewhiran C, Wisitsoraat A, Tuantranont A, Phanichphant S. NO2 sensing properties of flame-made MnOx-loaded ZnO-nanoparticle thick film. Sens Actuators B. 2014;204:239-249.

[469] Kim T, Sharp A, Guo B. Effect of synthesis condition and annealing on the sensitivity and stability of gas sensors made of Zn-doped $\gamma-\mathrm{Fe}_{2} \mathrm{O}_{3}$ particles. Korean J Chem Eng 2010;27(3):1003-1009.

[470] Geng B, Zhan F, Fang C, Yu N. A facile coordination compound precursor route to controlled synthesis of $\mathrm{Co}_{3} \mathrm{O}_{4}$ nanostructures and their room-temperature gas sensing properties. $\mathrm{J}$ Mater Chem 2008;18(41):4977-4984.

[471] Li W Y, Xu L N, Chen J. Co3O4 nanomaterials in lithium-ion batteries and gas sensors. Adv Funct Mater 2005;15(5):851-857.

[472] Gou X, Wang G, Yang J, Park J, Wexler D. Chemical synthesis, characterisation and gas sensing performance of copper oxide nanoribbons. J Mater Chem 2008;18(9):965-969.

[473] Zhang Y, He X, Li J, Zhang H, Gao X. Gas-sensing properties of hollow and hierarchical copper oxide 
microspheres. Sens Actuator B 2007;128(1):293-298.

[474] Schopf S O, Salameh S, Mädler L. Transfer of highly porous nanoparticle layers to various substrates through mechanical compression. Nanoscale 2013;5(9):3764-3772.

[475] Samerjai T, Tamaekong N, Liewhiranc C, Wisitsoraat $\mathrm{A} \mathrm{NO}_{2}$ gas sensing of flame-made Pt-loaded $\mathrm{WO}_{3}$ thick films. J Solid State Chem 2014;214:47-52.

[476] Wegner K, Vinati S, Piseri P, Antonini A, Zelioli A, Barborini, E, Milani, P. High-rate production of functional nanostructured films and devices by coupling flame spray pyrolysis with supersonic expansion. Nanotechnology,2012;23(18):185603.

[477] Jiang H, Lau M, Tellkamp VL, Lavemia EJ. Synthesis of Nanostructured Coatings by High-Velocity Oxygen-Fuel Thermal Spraying. Handbook of Nanostructured Materials and Nanotechnology, Five-Volume Set, 3, 159. 1999

[478] Tuominen M, Teisala H, Aromaa M, Stepien M, Mäkelä JM, Saarinen JJ, Toivakka M, Kuusipalo J. Creation of superhydrophilic surfaces of paper and board. J Adhes Sci Technol, 2014;28(8-9):864-879.

[479] McCormick, JR, Zhao B, Rykov SA, Wang H, Chen JG. Thermal stability of flame-synthesized anatase $\mathrm{TiO}_{2}$ nanoparticles. J Phys Chem B 2004;108(45):17398-17402.

[480] Han J, Chang H, Lee J, Chang H. The aggregation of thermally stable particles in a premixed flat flame aerosol reactor. Aerosol Sci Technol 2003;37(7):550-564.

[481] Gröhn AJ, Eggersdorfer ML, Pratsinis SE, Wegner K. On-line monitoring of primary and agglomerate particle dynamics. J Aerosol Sci 2014;73:1-13.

[482] Gröhn AJ. Scale-up of Flame Aerosol Synthesis by Computational Fluid Dynamics. PhD thesis, ETH Zürich, 2014.

[483] Zhao B, Yang Z, Johnston MV, Wang H, Wexler AS, Balthasar M, Kraft M. Measurement and numerical simulation of soot particle size distribution functions in a laminar premixed ethylene-oxygen-argon flame. Combust Flame 2003;133(1):173-188.

[484] Ober F, Mayer M, Büttner H, Ebert F. Aerosol Measurement in Low-Pressure Systems with Standard Scanning Mobility Particle Sizers. Part Part Syst Charact 2002;19(4):229-239.

[485] Whitby E, Hoshino M. Development of a low-pressure aerosol sampler. Rev Sci Instrum, 1995;66(7):3955-3965.

[486] Wang Y, Liu P, Fang J, Wang WN, Biswas P. Kinetics of sub-2 nm TiO2 particle formation in an aerosol reactor during thermal decomposition of titanium tetraisopropoxide. J Nanopart Res 2015;17(3):1-13.

[487] Fang J, Wang Y, Attoui M, Chadha TS, Ray JR, Wang WN, Jun YS, Biswas P, Measurement of sub-2 $\mathrm{nm}$ clusters of pristine and composite metal oxides during nanomaterial synthesis in flame aerosol reactors. Anal Chem 2014;86(15):7623-7529.

[488] Wang Y, Fang J, Attoui M, Chadha TS, Wang WN, Biswas P, Application of half mini DMA for sub 2 $\mathrm{nm}$ particle size distribution measurement in an electrospray and a flame aerosol reactor. J Aerosol Sci 2014;71:52-64.

[489] Roth P, Hospital A. Design and test of a particle mass spectrometer (PMS). J Aerosol Sci 1994;25(1):61-73.

[490] Scheckman JH, McMurry PH, Pratsinis SE. Rapid characterization of agglomerate aerosols by in situ mass-mobility measurements. Langmuir 2009;25(14):8248-8254. 
[491] Allendorf, MD, Bautista JR, Potkay E. Temperature measurements in a vapor axial deposition flame by spontaneous Raman spectroscopy. J Appl Phys 1989;66(10):5046-5051.

[492] Liu XF, Smith ME, Tse SD. In situ Raman characterization of nanoparticle aerosols during flame synthesis. Appl Phys B 2010;100(3):643-653.

[493] Zhang YY, Xiong G, Li SQ, Dong ZZ, Buckley SG, Stephen DT. Novel low-intensity phase-selective laser-induced breakdown spectroscopy of $\mathrm{TiO}_{2}$ nanoparticle aerosols during flame synthesis. Combust Flame 2013;160(3):725-733.

[494] Eckbreth AC. Laser diagnostics for combustion temperature and species. CRC Press. 1996;

[495] Engel SR, Koegler AF, Gao Y, Kilian D, Voigt M, Seeger T, Peukert W, Leipertz A. Gas phase temperature measurements in the liquid and particle regime of a flame spray pyrolysis process using $\mathrm{O}_{2}$-based pure rotational coherent anti-Stokes Raman scattering. Appl Optics 2012;51(25):6063-6075.

[496] Hwang JY, Gil YS, Kim JI, Choi M, Chung SH. Measurements of temperature and OH radical distributions in a silica generating flame using CARS and PLIF. J Aerosol Sci 2001;32(5):601-613.

[497] Kim JI, Hwang JY, Lee J, Choi M, Chung SH. Numerical and experimental study on silica generating counterflow diffusion flames. Int J Heat Mass Transf 2005;48(1):75-81.

[498] Best PE, Chien PL, Carangelo RM, Solomon PR, Danchak M, Ilovici I. Tomographic reconstruction of FT-IR emission and transmission spectra in a sooting laminar diffusion flame: species concentrations and temperatures. Combust Flame 1991;85(3):309-318.

[499] Morrison PW, Raghavan R, Timpone AJ, Artelt CP, Pratsinis, SE. In situ Fourier transform infrared characterization of the effect of electrical fields on the flame synthesis of $\mathrm{TiO}_{2}$ particles. Chem Mat 1997;9(12):2702-2708.

[500] Schulz H., Mädler L, Strobel R, Jossen R, Pratsinis SE, Johannessen T. Independent control of metal cluster and ceramic particle characteristics during one-step synthesis of $\mathrm{Pt} / \mathrm{TiO}_{2}$. J Mater Res 2005;20(09):2568-2577.

[501] Rudin T, Pratsinis SE. Homogeneous iron phosphate nanoparticles by combustion of sprays. Ind Eng Chem Res 2012;51(23):7891-7900.

[502] Glumac NG, Chen YJ, Skandan G. Diagnostics and modeling of nanopowder synthesis in low pressure flames. J Mater Res 1998;13(09):2572-2579.

[503] Markham JR., Zhang YP, Carangelo RM, \& Solomon PR. FT-IR emission/transmission tomography of a coal flame. In Symposium (International) on Combustion. 1991;23(1):1869-1875.

[504] Kronemayer H, Ifeacho P, Hecht C, Dreier T, Wiggers H, Schulz C. Gas-temperature imaging in a low-pressure flame reactor for nano-particle synthesis with multi-line NO-LIF thermometry. Appl Phys B 2007;88(3):373-377.

[505] Hecht C, Kronemayer H, Dreier T, Wiggers H, Schulz C. Imaging measurements of atomic iron concentration with laser-induced fluorescence in a nanoparticle synthesis flame reactor. Appl Phys B 2009;94(1):119-125.

[506] Bailey S, Glumac NG. Laser-induced-fluorescence detection of $\mathrm{SnO}$ in low-pressure particle-synthesis flames. Appl Phys B.2003;77(4):455-461.

[507] Glumac NG. Formation and consumption of $\mathrm{SiO}$ in powder synthesis flames. Combust Flame 2001;124(4):702-711.

[508] McMillin BK, Biswas P, Zachariah MR. In situ characterization of vapor phase growth of iron 
oxide-silica nanocomposites: Part I. 2-D planar laser-induced fluorescence and Mie imaging. J Mater Res 1996;11(06):1552-1561.

[509] Biswas P, Zachariah MR. In situ immobilization of lead species in combustion environments by injection of gas phase silica sorbent precursors. Environ Sci Technol 1997;31(9):2455-2463.

[510] Colibaba-Evulet A, Singhal A, Glumac, N. Detection of AIO and TiO by Laser-Induced Fluorescence in Powder Synthesis Flames. Combust Sci Technol 2000;157(1):129-139.

[511] Muramoto J, Inmaru T, Nakata Y, Okada T, Maeda M. Spectroscopic imaging of nanoparticles in laser ablation plume by redecomposition and laser-induced fluorescence detection. Appl Phys Lett 2000;77(15):2334-2336.

[512] Lee J, Olfert J, Altman IS, Choi M. Determination of particle temperatures in a silica-generating counterflow flame via flame emission measurements. Int J Heat Mass Transf 2010;53(1):564-567.

[513] Burkert A, Müller D, Paa W. Si and SiO detection in a HMDSO/propane/air flame using spatially resolved optical emission spectroscopy (OES). J. Quant. Spectrosc. Radiat Transf 2013;114:101-108.

[514] Kulkarni P, Baron PA, Willeke K. Aerosol measurement: principles, techniques, and applications. 2011 John Wiley \& Sons.

[515] Graham SC, Homer JB. Coagulation of molten lead aerosols. Faraday Symp Chem Soc 1973;7:85-96.

[516] Yang G, Biswas P. Study of the sintering of nanosized titania agglomerates in flames using in situ light scattering measurements. Aerosol Sci Technol 1997;27(4):507-521.

[517] Velazco-Roa MA, Thennadil SN. Estimation of complex refractive index of polydisperse particulate systems from multiple-scattered ultraviolet-visible-near-infrared measurements. Appl Optics 2007;46(18):3730-3735.

[518] Ma L, Hanson RK. Measurement of aerosol size distribution functions by wavelength-multiplexed laser extinction. Appl Phys B 2005;81(4):567-576.

[519] Beretta F, Cavaliere A, Allessio A. Drop size and concentration in a spray by sideward laser light scattering measurements. Combust Sci Technol 1984;36(1-2):19-37.

[520] Ma L. Measurement of aerosol size distribution function using Mie scattering-Mathematical considerations. J Aerosol Sci 2007;38(11):1150-1162.

[521] Sorensen CM. Light scattering by fractal aggregates: a review. Aerosol Sci Technol 2001;35(2):648-687.

[522] Feigin LA, Svergun DI. Structure analysis by small-angle X-ray and neutron scattering. G. W. Taylor (Ed.). New York: Plenum Press; 1987. pp. 68-73

[523] Hyeon-Lee J, Beaucage G, Pratsinis SE, Vemury S. Fractal analysis of flame-synthesized nanostructured silica and titania powders using small-angle X-ray scattering. Langmuir 1998;14(20):5751-5756.

[524] Beaucage G, Kammler HK, Mueller R, Strobel R, Agashe N, Pratsinis SE, Narayanan T. Probing the dynamics of nanoparticle growth in a flame using synchrotron radiation. Nat Mater 2004;3(6):370-373.

[525] Camenzind A, Schulz H, Teleki A, Beaucage G, Narayanan T, Pratsinis SE. Nanostructure evolution: from aggregated to spherical $\mathrm{SiO} 2$ particles made in diffusion flames. Eur J Inorg Chem 2008;6:911-918.

[526] Zachariah MR, Chin D, Semerjian HG, Katz JL. Dynamic light scattering and angular dissymmetry for the in situ measurement of silicon dioxide particle synthesis in flames. Appl Optics 1989;28(3):530-536.

[527] Heine MC, Pratsinis SE. Droplet and particle dynamics during flame spray synthesis of nanoparticles. Ind Eng Chem Res 2005;44(16):6222-6232. 
[528] Bassi AL, Cattaneo D, Russo V, Bottani CE, Barborini E, Mazza T, Piseri P, Milani P, Ernst FO, Wegner K, Pratsinis SE. Raman spectroscopy characterization of titania nanoparticles produced by flame pyrolysis: the influence of size and stoichiometry. J Appl Phys 2005;98(7):074305.

[529] Shaddix CR, Smyth KC. Laser-induced incandescence measurements of soot production in steady and flickering methane, propane, and ethylene diffusion flames. Combust Flame 1996;107(4):418-452.

[530] Mewes B, Seitzman JM. Soot volume fraction and particle size measurements with laser-induced incandescence. Appl Optics 1997;36(3):709-717.

[531] Michelsen HA, Liu F, Kock BF, Bladh H, Boïarciuc A, Charwath M, Dreier T, Hadef R, Hofmann M, Reimann J, Will S, Bengtsson PE, Bockhorn H, Foucher F, Geigle KP, Mounaïm-rousselle C, Schulz C, Stirn R, Tribalet B, Suntz R. Modeling laser-induced incandescence of soot: a summary and comparison of LII models. Appl Phys B 2007;87(3):503-521.

[532] Filippov AV, Markus MW, Roth P. In-situ characterization of ultrafine particles by laser-induced incandescence: sizing and particle structure determination. J Aerosol Sci 1999;30(1):71-87.

[533] Starke R, Kock B, Roth P. Nano-particle sizing by laser-induced-incandescence (LII) in a shock wave reactor. Shock Waves 2003;12(5):351-360.

[534] Eremin A, Gurentsov E, Popova E, Priemchenko K. Size dependence of complex refractive index function of growing nanoparticles. Appl Phys B 2011;104(2):285-295.

[535] Sipkens T, Joshi G, Daun K J, Murakami Y. Sizing of Molybdenum Nanoparticles Using Time-Resolved Laser-Induced Incandescence. J Heat Transf 2013;135(5):052401.

[536] Eremin A, Gurentsov E, Schulz C. Influence of the bath gas on the condensation of supersaturated iron atom vapour at room temperature. J Phys D-Appl Phys 2008;41(5):055203.

[537] Lehre T, Suntz R, Bockhorn H. Time-resolved two-color LII: size distributions of nano-particles from gas-to-particle synthesis. Proc Combust Inst 2005;30(2):2585-2593.

[538] Cignoli F, Bellomunno C, Maffi S, Zizak G. Laser-induced incandescence of titania nanoparticles synthesized in a flame. Appl Phys B 2009;96(4):593-599.

[539] Vander Wal RL, Ticich TM, West JR. Laser-induced incandescence applied to metal nanostructures. Appl Optics 1999;38(27):5867-5879.

[540] Murakam Y, Sugatani T, Nosaka Y. Laser-induced incandescence study on the metal aerosol particles as the effect of the surrounding gas medium. J Phys Chem A 2005;109(40):8994-9000.

[541] Maffi S, Cignoli F, Bellomunno C, De Iuliis S, Zizak G. Spectral effects in laser induced incandescence application to flame-made titania nanoparticles. Spectroc Acta Pt B-Atom Spectr 2008;63(2):202-209.

[542] Sipkens TA., Mansmann R, Daun KJ, Petermann N, Titantah JT, Karttunen M, Wiggers H, Dreier T, Schulz C. In situ nanoparticle size measurements of gas-borne silicon nanoparticles by time-resolved laser-induced incandescence. Appl Phys B 2014;116(3):623-636.

[543] Amodeo T, Dutouquet C, Tenegal F, Guizard B, Maskrot H, Le Bihan O, Frejafon E. On-line monitoring of composite nanoparticles synthesized in a pre-industrial laser pyrolysis reactor using Laser-Induced Breakdown Spectroscopy. Spectroc Acta Pt B-Atom Spectr 2008;63(10):1183-1190.

[544] Mukherjee D, Rai A, Zachariah MR. Quantitative laser-induced breakdown spectroscopy for aerosols via internal calibration: Application to the oxidative coating of aluminum nanoparticles. J Aerosol Sci 2006;37(6):677-695.

[545] Tsantilis S, Pratsinis SE, Haas V. Simulation of synthesis of palladium nanoparticles in a jet aerosol 
flow condenser. J Aerosol Sci 1999;30:785-803.

[546] Rosner DE, Pyykönen JJ. Bivariate moment simulation of coagulating and sintering nanoparticles in flames. AIChE J 2002;48:476-491.

[547] D’Alessio A, Barone AC, Cau R, D’Anna A, Minutolo P. Surface deposition and coagulation efficiency of combustion generated nanoparticles in the size range from 1 to $10 \mathrm{~nm}$. Proc Combust Inst 2005;30:2595-2603.

[548] Kobata A, Kusakabe K, Morooka S. Growth and transformation of $\mathrm{TiO}_{2}$ crystallites in aerosol reactor. AIChE J 1991;37:347-359.

[549] Zachariah MR, Dimitriou P. Controlled Nucleation in Aerosol Reactors for Suppression of Agglomerate Formation: A Numerical Study. Aerosol Sci Technol 1990;13:413-425.

[550] Ramkrishna D, Singh MR. Population Balance Modeling: Current Status and Future Prospects. Annu Rev Chem Biomol Eng 2014;5:123-146.

[551] Kelton K, Greer AL. Nucleation in condensed matter: applications in materials and biology. Vol. 15. Elsevier, 2010.

[552] Smoluchowski MV. Versuch einer mathematischen theorie der koagulationskinetic kolloide osunger. Z Phys Chemie-Int J Res 1917;92:129.

[553] Friedlander S K. Smoke, Dust and Haze: Fundamentals of Aerosol Dynamics. 2nd edition. New York: Oxford University Press, 200033.

[554] Lee MH. A survey of numerical solutions to the coagulation equation. J Phys A: Math Gen 2001;34:10219.

[555] Alexopoulos AH, Kiparissides CA. Part II: Dynamic evolution of the particle size distribution in particulate processes undergoing simultaneous particle nucleation, growth and aggregation. Chem Eng Sci 2005;60:4157-4169.

[556] McGraw R. Description of Aerosol Dynamics by the Quadrature Method of Moments. Aerosol Sci Technol 1997;27:255-265.

[557] Frenklach M. Method of moments with interpolative closure. Chem Eng Sci 2002;57:2229-2239.

[558] Wei J, Kruis FE. A GPU-based parallelized Monte-Carlo method for particle coagulation using an acceptance-rejection strategy. Chem Eng Sci 2013;104:451-459.

[559] Zhao H, Kruis FE, Zheng C. Reducing statistical noise and extending the size spectrum by applying weighted simulation particles in Monte Carlo simulation of coagulation. Aerosol Sci Technol 2009;43(8):781-793.

[560] Rigopoulos S. Population balance modelling of polydispersed particles in reactive flows. Prog Energy Combust Sci 2010;36:412-443.

[561] Prakash A, Bapat AP, Zachariah MR. A simple numerical algorithm and software for solution of nucleation, surface growth, and coagulation problems. Aerosol Sci Technol 2003;37:892-898.

[562] Mukherjee D, Prakash A, Zachariah MR. Implementation of a discrete nodal model to probe the effect of size-dependent surface tension on nanoparticle formation and growth. J Aerosol Sci 2006;37:1388-1399.

[563] Johannessen T, Pratsinis SE, Livbjerg H. Computational fluid-particle dynamics for the flame synthesis of alumina particles. Chem Eng Sci 2000;55:177-191.

[564] Mühlenweg H, Gutsch A, Schild A, Pratsinis SE. Process simulation of gas-to-particle-synthesis via population balances: Investigation of three models. Chem Eng Sci 2002;57:2305-22. 
[565] Rosner DE, McGraw R, Tandon P. Multivariate Population Balances via Moment and Monte Carlo Simulation Methods: An Important Sol Reaction Engineering Bivariate Example and "Mixed" Moments for the Estimation of Deposition, Scavenging, and Optical Properties for Populations of Nonspherical Suspended Particles. Ind Eng Chem Res 2003;42:2699-2711.

[566] Rosner DE. Improved rate laws and population balance simulation methods; CRE applications, including the combustion synthesis of valuable nano-particles. Int J Chem React Eng 2006;4.D

[567] Koch W, Friedlander SK. The effect of particle coalescence on the surface area of a coagulating aerosol. J Colloid Interface Sci 1990;140:419-427.

[568] Akroyd J, Smith AJ, Shirley R, McGlashan LR, Kraft M. A coupled CFD-population balance approach for nanoparticle synthesis in turbulent reacting flows. Chem Eng Sci 2011;66:3792-805.

[569] Mehta M, Raman V, Fox RO. On the role of gas-phase and surface chemistry in the production of titania nanoparticles in turbulent flames. Chem Eng Sci 2013:104:1003-1018.

[570] Raman V, Fox RO. Modeling of fine-particle formation in turbulent flames. Annu Rev Fluid Mech 2016:48:159-190.

[571] Shmakov AG, Korobeinichev OP, Knyazkov DA, Paletsky AA, Maksutov RA, Gerasimov IE, Bolshova TA, Kiselev VG, Gritsan NP. Combustion chemistry of $\mathrm{Ti}\left(\mathrm{OC}_{3} \mathrm{H}_{7}\right)_{4}$ in premixed flat burner-stabilized $\mathrm{H}_{2} / \mathrm{O}_{2} / \mathrm{Ar}$ flame at 1atm. Proc Combust Institute 2013:34(1):1143-1149.

[572] Okuyama K, Ushio R, Kousaka Y, Flagan RC, Seinfeld JH. Particle generation in a chemical vapor deposition process with seed particles. AIChE J 1990;36(3):409-419.

[573] Seto T, Shimada M, Okuyama K. Evaluation of sintering of nanometer-sized titania using aerosol method. Aerosol Sci Technol 1995;23(2):183-200.

[574] Battiston GA, Gerbasi R, Porchia M, Gasparotto A. Metal Organic CVD of Nanostructured Composite TiO2-Pt Thin Films: A Kinetic Approach. Chem Vapor Depos 1999;5(1):13-20.

[575] Kuwana K, Saito K. Modeling ferrocene reactions and iron nanoparticle formation: Application to CVD synthesis of carbon nanotubes. Proc Combust Inst 2007;31(2):1857-1864.

[576] Bhattacharjee A, Rooj A, Roy D, Roy M. Thermal Decomposition Study of Ferrocene $\left[\left(\mathrm{C}_{5} \mathrm{H}_{5}\right)_{2} \mathrm{Fe}\right]$. J Exp Phy 2014;2014.

[577] Galembeck A, Alves OL. $\mathrm{BiVO}_{4}$ thin film preparation by metalorganic decomposition. Thin Solid Films 2000;365(1):90-93.

[578] Zhao RY, Dong P, Liang WJ. Study of hydrolysis kinetics of tetraethyl orthosilicate in the preparation of monodisperse silica system. Acta Phys-Chim Sin 1995;11(7):612-616

[579] Menz WJ, Kraft M. A new model for silicon nanoparticle synthesis. Combust Flame 2013;160:947-958.

[580] Marlow WH. Derivation of aerosol collision rates for singular attractive contact potentials. J Chem Phys 1980;73(12):6284-6287.

[581] Marlow WH. Lifshitz-van der Waals forces in aerosol particle collisions. I. Introduction: water droplets. The J Chem Phys 1980;73(12):6288-6295.

[582] Kerminen VM. Simulation of Brownian coagulation in the presence of van der Waals forces and viscous interactions. Aerosol Sci Technol 1994;20(2):207-214.

[583] Alam MK. The effect of van der Waals and viscous forces on aerosol coagulation. Aerosol Sci Technol $1987 ; 6(1), 41-52$.

[584] Marlow WH Lead aerosol brownian collision rates at normal and elevated temperature: theory. J Colloid 
Interface Sci 1982;87(1):209-215.

[585] Arunachalam V, Lucchese RR, Marlow WH. Simulations of aerosol aggregation including long-range interactions. Phys Rev E 1999;60(2):2051.

[586] Fuchs NA, The Mechanics of Aerosols (Pergamon, New York, 1964).

[587] Ouyang H, Gopalakrishnan R, Hogan Jr CJ. Nanoparticle collisions in the gas phase in the presence of singular contact potentials. J Chem Phys 2012;137(6):064316.

[588] Blanton SA, Leheny RL, Hines MA, Guyot-Sionnest P. Dielectric dispersion measurements of CdSe nanocrystal colloids: observation of a permanent dipole moment. Phys Rev Lett 1997;79(5):865.

[589] Shim M, Guyot-Sionnest P. Permanent dipole moment and charges in colloidal semiconductor quantum dots. J Chem Phys 1999;111(15):6955-6964.

[590] Yan W, Li S, Zhang Y, Yao Q, Tse SD. Effects of dipole moment and temperature on the interaction dynamics of titania nanoparticles during agglomeration. J Phys Chem C 2010;114(24):10755-10760.

[591] Zhang YY, Li SQ, Yan W, Yao Q, Tse SD. Role of dipole-dipole interaction on enhancing Brownian coagulation of charge-neutral nanoparticles in the free molecular regime. J Chem Phys 2012;134(8):084501.

[592] Koparde VN, Cummings PT. Molecular dynamics simulation of titanium dioxide nanoparticle sintering. J Phys Chem B 2005;109(51):24280-24287.

[593] Zhang Y, Li S, Yan W, Stephen DT. Effect of size-dependent grain structures on the dynamics of nanoparticle coalescence. J Appl Phys 2012;111(12):124321.

[594] Iijima S, Ajayan PM. Substrate and size effects on the coalescence of small particles. J Appl Phys 1991;70(9):5138-5140.

[595] Arcidiacono S, Bieri NR, Poulikakos D, Grigoropoulos CP. On the coalescence of gold nanoparticles. Int J Multiph Flow 2004;30(7):979-994.

[596] Zhu H, Averback RS. Sintering of nano-particle powders: simulations and experiments. Mater Manuf Process 1996;11(6):905-923.

[597] Lehtinen KE, Zachariah MR. Effect of coalescence energy release on the temporal shape evolution of nanoparticles. Phys Rev B 2001;63(20):205402.

[598] J. Frenkel. Viscous flow on crystalline bodies under the action of surface tension. J Phys 1945;9:385-391.

[599] Friedlander SK, Wu MK. Linear rate law for the decay of the excess surface area of a coalescing solid particle. Phys Rev B 1994;49(5):3622.

[600] Koparde VN, Cummings PT. Phase transformations during sintering of titania nanoparticles. ACS nano 2008;2(8):1620-1624.

[601] Naicker PK, Cummings PT, Zhang H, Banfield JF. Characterization of titanium dioxide nanoparticles using molecular dynamics simulations. J Phys Chem B 2005;109(32):15243-15249.

[602] Zhang H, Banfield JF. Structural characteristics and mechanical and thermodynamic properties of nanocrystalline $\mathrm{TiO}_{2}$. Chem Rev 2014;114(19):9613-9644.

[603] Ding XZ, Liu XH. Grain growth enhanced by anatase-to-rutile transformation in gel-derived nanocrystalline titania powders. J Alloy Compd 1997;248(1):143-145.

[604] Zhou Y, Fichthorn KA. Microscopic view of nucleation in the anatase-to-rutile transition. J Phys Chem C 2012;116:8314.

[605] Hansen TW, DeLaRiva AT, Challa, SR, Datye AK. Sintering of catalytic nanoparticles: particle 
migration or ostwald ripening? Accounts Chem Res 2013;46(8):1720-1730.

[606] Campbell CT. The energetics of supported metal nanoparticles: relationships to sintering rates and catalytic activity. Accounts Chem Res 2013;46(8):1712-1719.

[607] Ostwald W. Periodische Erscheinungen bei der Auflösung des Chroms in Säuren-Zweite Mitteilung. Z Phys Chem 1990;35:200-256.

[608] Kalikmanov VI, Wölk J, Kraska T. Argon nucleation: Bringing together theory, simulations, and experiment. J Chem Phys 2008;128(12):124506.

[609] Li SQ, Marshall JS, Liu GQ, YaoQ. Adhesive particulate flow: The discrete-element method and its application in energy and environmental engineering, Prog Energy Combust Sci 2011;37:633-668.

[610] Marshall JS, Li SQ. Adhesive particle flow: a discrete-element approach, in Chap. 9. Cambridge University Press, 2014.

[611] Wenning L, Müser MH. Friction laws for elastic nanoscale contacts. Europhys Lett 2001;54(5):693-699.

[612] Luan B, Robbins MO. The breakdown of continuum models for mechanical contacts. Nat Lett 2005;435:929-932.

[613] Sun WF, Zeng QH, Yu AB, Kendall K. Calculation of normal contact forces between silica nanospheres. Langmuir 2013;29:7825-7837.

[614] Higashitani K, Iimura K, Sanda H. Simulation of deformation and breakup of large aggregates in flows of viscous fluids. Chem Eng Sci 2001;56:2927-2938.

[615] Eggersdorfer ML, Kadau D, Herrmann HJ, Pratsinis SE. Fragmentation and restructuring of soft-agglomerates under shear. J Colloid Interface Sci 2010;342:261-268.

[616] Goudeli E, Eggersdorfer ML, Pratsinis SE. Coagulation-agglomeration of fractal-like particles: structure and self-preserving size distribution. Langmuir 2015;31:1320-1327.

[617] Eggersdorfer ML, Pratsinis SE. Restructuring of aggregates and their primary particle size distribution during sintering. AIChE J 2013;59:1118-1125.

[618] Luding S, Manetsberger K, Müllers J. A discrete model for long time sintering. J Mech Phys Solids 2005;53:455-491.

[619] Dong Z, Al-Sharab JF, Kear BH, Tse SD. Combined Flame and Electrodeposition Synthesis of Energetic Coaxial Tungsten-Oxide/Aluminum Nanowire Arrays. Nano Lett 2013;13(9):4346-4350. 


\section{Appendix}

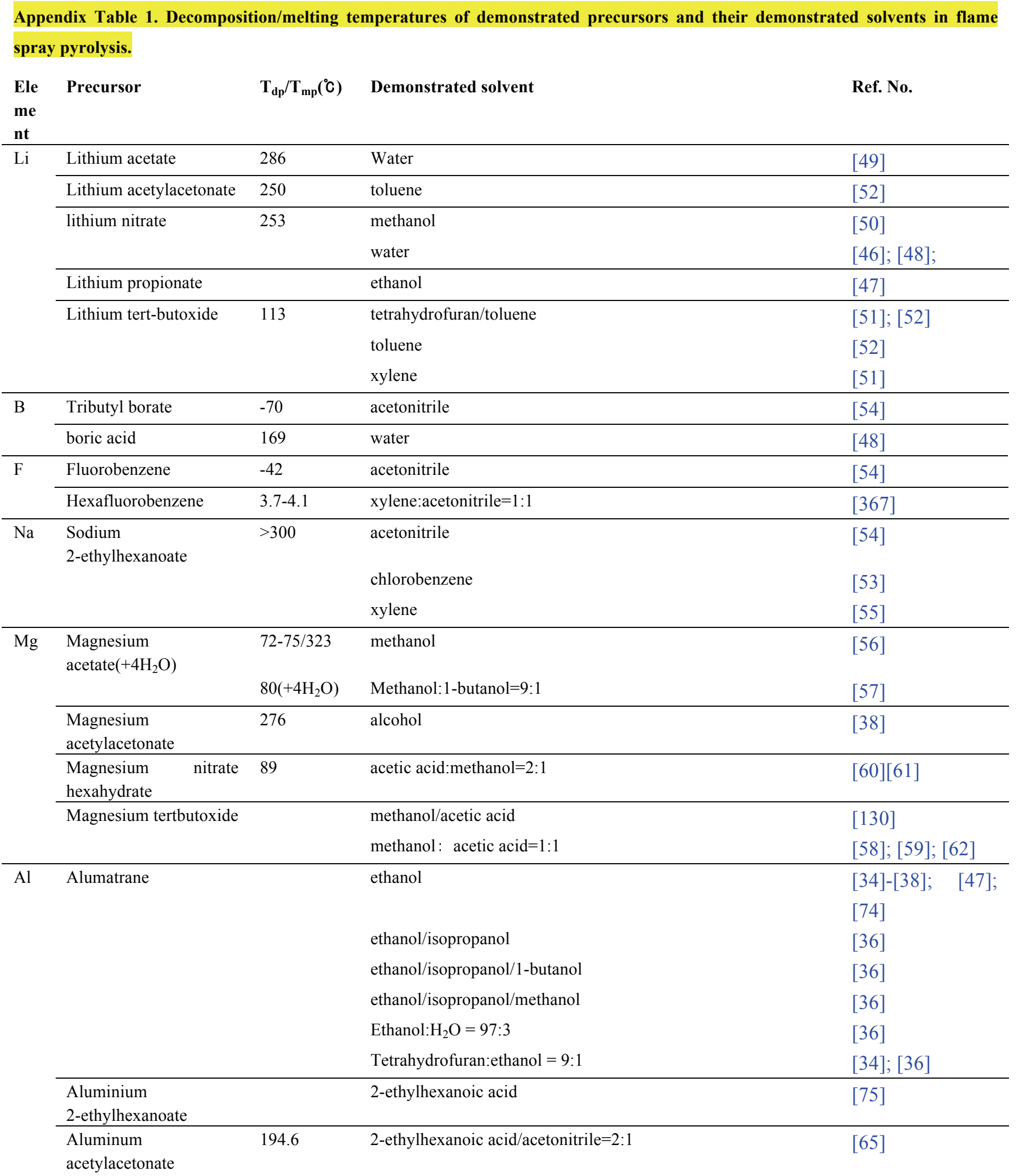




\begin{tabular}{|c|c|c|c|c|}
\hline \multirow[t]{23}{*}{$\begin{array}{l}\text { Ele } \\
\text { me } \\
\text { nt }\end{array}$} & Precursor & $\mathbf{T}_{\mathrm{dp}} / \mathbf{T}_{\mathrm{mp}}\left({ }^{\circ} \mathrm{C}\right)$ & Demonstrated solvent & Ref. No. \\
\hline & & & acetic acid:methanol $=2: 1$ & {$[60] ;[61]$} \\
\hline & & & ethanol & {$[34] ;[36]$} \\
\hline & & & methanol/acetic acid mixture & {$[130]$} \\
\hline & & & Methanol:acetic acid=1:1 & {$[58] ;[59] ;[62] ;$} \\
\hline & & & & {$[72] ;[73] ;[347]$} \\
\hline & & & o-xylene & {$[101]$} \\
\hline & & & s-butanol $/$ xylene/methanol:acetic acid=1:1 & {$[68]$} \\
\hline & & & tetrahydrofuran & / \\
\hline & & & toluene & {$[210]$} \\
\hline & Aluminum butoxide & $103-110$ & xylene & [354] \\
\hline & Aluminium & $138-142$ & carboxylic acids(C1-C3) & \\
\hline & & & linear alcohols $(\mathrm{C} 1-\mathrm{C} 8)$ & {$[63]$} \\
\hline & & & xylene/ethylacetate $=65: 35$ & {$[70]$} \\
\hline & $\begin{array}{l}\text { Aluminium } \\
\text { nitrate }\left(+9 \mathrm{H}_{2} \mathrm{O}\right)\end{array}$ & $73\left(+9 \mathrm{H}_{2} \mathrm{O}\right)$ & 1-butanol & {$[34] ;[36]$} \\
\hline & & & carboxylic acids(C1-C3) & \\
\hline & & & ethanol & [34]; [36] \\
\hline & & & linear alcohols $(\mathrm{C} 1-\mathrm{C} 8)$ & {$[63]$} \\
\hline & & & water & [372] \\
\hline & $\begin{array}{l}\text { Aluminum } \\
\text { tri-secbutoxide }\end{array}$ & & diethyleneglycol monobutyl ether:acetic anhydride $=2: 1$ & {$[66] ;[67] ;[205]$} \\
\hline & & & xylene & {$[68] ;[71]$} \\
\hline & & & xylene: acetonitrile $=2: 1$ & {$[69]$} \\
\hline & & & xylene: acetonitrile $=7: 3$ & [347] \\
\hline \multirow[t]{13}{*}{$\mathrm{Si}$} & $\begin{array}{ll}\text { Colloidal } & \mathrm{SiO}_{2} \\
\text { nanoparticles }\end{array}$ & 1610 & water & [189] \\
\hline & $\begin{array}{l}\text { Hexamethyldisiloxane } \\
\text { (HMDSO) }\end{array}$ & -66 & 2-ethylhexanoic acid/acetonitrile $=2: 1$ & {$[65]$} \\
\hline & & & diethyleneglycol monobutyl ether: 2 -ethylhexanoic acid $=1: 1$ & [89] \\
\hline & & & diethylene glycol monobutyl ether:ethanol=1:1 & [465] \\
\hline & & & ethanol & {$[41] ;[327] ;[495]$} \\
\hline & & & iso-octane & {$[41]$} \\
\hline & & & methanol & {$[41]$} \\
\hline & & & Methanol:1-butanol=9:1 & {$[57]$} \\
\hline & & & Methanol:acetic acid=94:6 & {$[45]$} \\
\hline & & & Xylene & {$[55] ;[81] ;[463]$} \\
\hline & $\begin{array}{l}\text { Tetraethoxysilane } \\
\text { (TEOS) }\end{array}$ & -82.5 & 2-ethylhexanoic acid/touluene mixture & [75] \\
\hline & & & alcohol, carboxylic acid, or a mixture & [76]; [77] \\
\hline & & & Ethanol & {$[47] ;[81] ;[476]$} \\
\hline
\end{tabular}




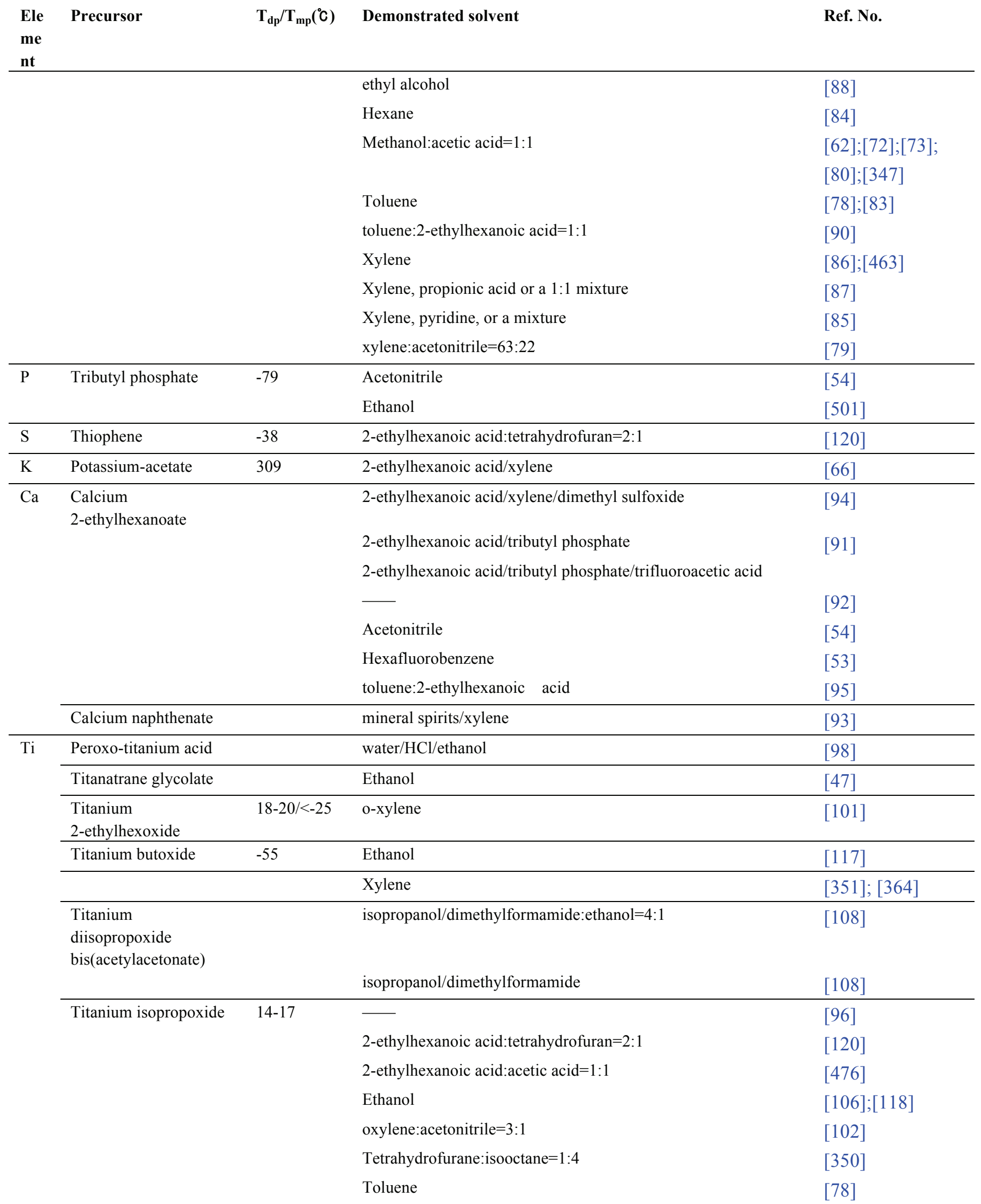




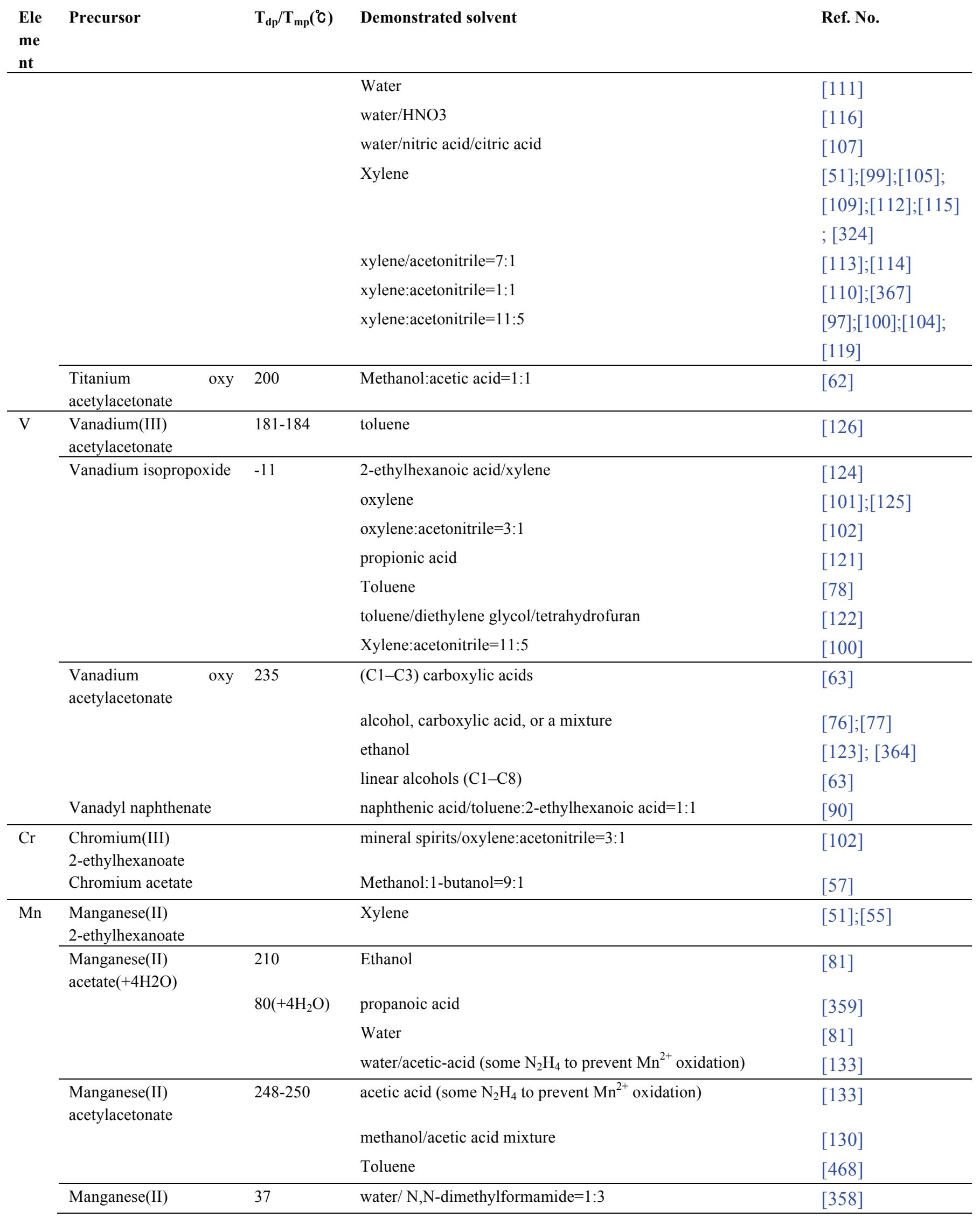




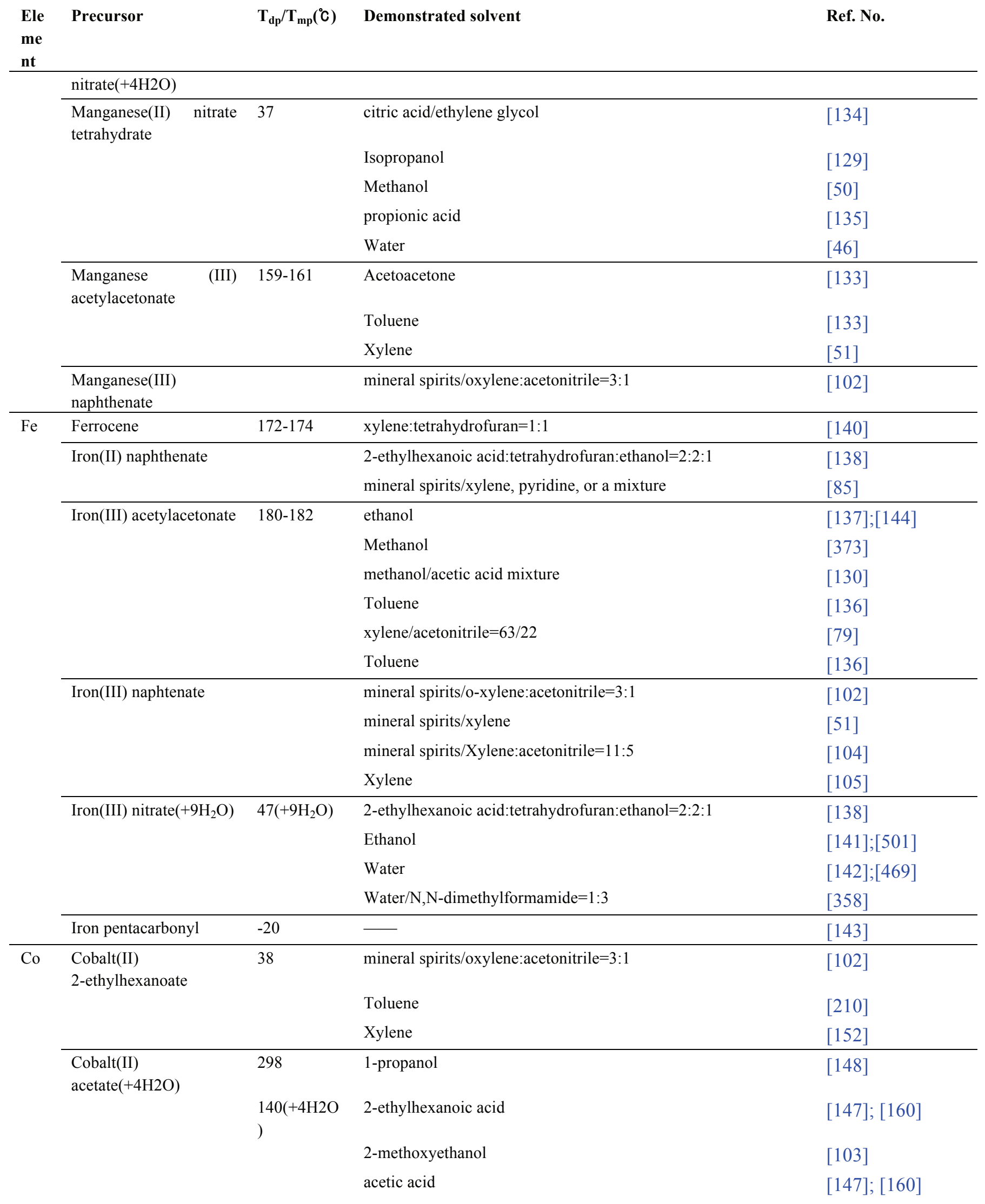




\begin{tabular}{|c|c|c|c|c|}
\hline \multirow[t]{17}{*}{$\begin{array}{l}\text { Ele } \\
\text { me } \\
\text { nt }\end{array}$} & \multirow[t]{10}{*}{ Precursor } & $\mathbf{T}_{\mathrm{dp}} / \mathbf{T}_{\mathbf{m p}}\left({ }^{\circ} \mathrm{C}\right)$ & Demonstrated solvent & Ref. No. \\
\hline & & & butyric acid & [147]; [160] \\
\hline & & & Ethanol & {$[148]$} \\
\hline & & & hexanoic acid & {$[147] ;[160]$} \\
\hline & & & Methanol & {$[148]$} \\
\hline & & & $\begin{array}{l}\text { methanol:propionic acid=1:1; ethanol:propionic acid=1:1; } \\
\text { 1-propanol:propionic acid=1:1; propionic acid/1-octanol; } \\
\text { propionic acid/1-pentanol } \\
\text { octanoic acid }\end{array}$ & [148] \\
\hline & & & propionic acid & $\begin{array}{l}{[146] ; \quad[147] ;} \\
{[151]}\end{array}$ \\
\hline & & & propionic acid:1-propanol:water $=5: 4: 1$ & [149]; [150] \\
\hline & & & Xylene & {$[86]$} \\
\hline & & & water/niric acid/citric acid & [360] \\
\hline & $\begin{array}{l}\text { Cobalt(II) } \\
\text { acetylacetonate }\end{array}$ & $165-170$ & xylene:tetrahydrofuran=1:1 & {$[140]$} \\
\hline & Cobalt naphthenate & & mineral spirits/xylene & [351]; [361] \\
\hline & $\begin{array}{l}\text { Cobalt(II) } \\
\text { nitrate }(+4 \mathrm{H} 2 \mathrm{O},+6 \mathrm{H} 2 \mathrm{O})\end{array}$ & 100 & 1-propanol & {$[148]$} \\
\hline & & $55(+6 \mathrm{H} 2 \mathrm{O})$ & Ethanol & [141]; [148] \\
\hline & & & Methanol & [148] \\
\hline & & & $\begin{array}{l}\text { methanol:propionic acid=1:1; ethanol:propionic acid=1:1; } \\
\text { 1-propanol:propionic acid=1:1; propionic acid/1-octanol; } \\
\text { propionic acid/1-pentanol }\end{array}$ & {$[148]$} \\
\hline & & & Water & {$[46] ;[48]$} \\
\hline & $\begin{array}{l}\text { Cobalt(III) } \\
\text { acetylacetonate }\end{array}$ & $210-213$ & methanol/acetic acid mixture & {$[130]$} \\
\hline & Cobalt(III) propionate & & water/propionic acid & {$[153]$} \\
\hline \multirow[t]{8}{*}{$\mathrm{Ni}$} & $\begin{array}{l}\text { Nickel(II) } \\
\text { 2-ethylhexanoate } \\
\end{array}$ & & Tetrahydrofurane & {$[155]$} \\
\hline & $\begin{array}{l}\text { Nickel(II) } \\
\text { acetate }(+4 \mathrm{H} 2 \mathrm{O})\end{array}$ & 310 & acetic acid & {$[133] ;[160]$} \\
\hline & & $160\left(+4 \mathrm{H}_{2} \mathrm{O}\right)$ & Methanol & {$[56]$} \\
\hline & & & propionic acid/xylene & {$[87]$} \\
\hline & $\begin{array}{l}\text { Nickel(II) } \\
\text { nitrate }\left(+6 \mathrm{H}_{2} \mathrm{O}\right)\end{array}$ & $56\left(+6 \mathrm{H}_{2} \mathrm{O}\right)$ & aqueous nitric acid solution & {$[154]$} \\
\hline & & & Water & {$[46]$} \\
\hline & Nickel(II) propionate & & water/propionic acid & {$[153]$} \\
\hline & Nickel(III) naphthenate & & toluene/o-xylene:acetonitrile=3:1 & {$[102]$} \\
\hline $\mathrm{Cu}$ & Colloidal $\quad \mathrm{CuO}$ & 1326 & Water & {$[167]$} \\
\hline
\end{tabular}




\begin{tabular}{|c|c|c|c|c|}
\hline \multirow[t]{14}{*}{$\begin{array}{l}\text { Ele } \\
\text { me } \\
\text { nt }\end{array}$} & Precursor & $\mathbf{T}_{\mathrm{dp}} / \mathbf{T}_{\mathrm{mp}}\left({ }^{\circ} \mathrm{C}\right)$ & Demonstrated solvent & Ref. No. \\
\hline & \multicolumn{4}{|l|}{ nanoparticles } \\
\hline & \multirow{4}{*}{$\begin{array}{l}\text { Copper } \\
\text { 2-ethylhexanoate }\end{array}$} & 252 & o-xylene:acetonitrile $=3: 1$ & {$[102]$} \\
\hline & & & & \\
\hline & & & Xylene & {$[109] ;[162]-[164]$} \\
\hline & & & xylene:acetonitrile $=1: 1$ & {$[367]$} \\
\hline & Copper acetate & 115 & Xylene & {$[86]$} \\
\hline & \multirow{6}{*}{$\begin{array}{l}\text { Copper } \\
\text { nitrate }\left(+2.5 \mathrm{H}_{2} \mathrm{O},+3 \mathrm{H}_{2} \mathrm{O}\right. \\
\text { ) }\end{array}$} & $\begin{array}{l}114.5\left(+3 \mathrm{H}_{2}\right. \\
\text { O) }\end{array}$ & acetic acid:methanol $=2: 1$ & {$[60][61]$} \\
\hline & & & & \\
\hline & & & Ethanol & {$[161]$} \\
\hline & & & Isopropanol & {$[168]$} \\
\hline & & & Water & [49]; [157] \\
\hline & & & water/citric acid(as the chelating agent) & {$[166]$} \\
\hline & Copper propionate & & water/propionic acid & {$[153]$} \\
\hline \multirow[t]{16}{*}{$\mathrm{Zn}$} & \multirow[t]{2}{*}{ Zinc 2-ethylhexanoate } & & Ethanol & [81] \\
\hline & & & Water & [81] \\
\hline & Zinc acetate & $83-86$ & Water & [338] \\
\hline & $\begin{array}{l}\text { Zinc } \\
\text { acetylacetonate }\left(+\mathrm{H}_{2} \mathrm{O}\right)\end{array}$ & $\begin{array}{l}135-138 \\
\left(+\mathrm{H}_{2} \mathrm{O}\right)\end{array}$ & 2-Methoxyethanol/acetic acid & [173] \\
\hline & Zinc acrylate & $240-244$ & Methanol: acetic acid $=94: 6$ & {$[44] ;[45]$} \\
\hline & \multirow[t]{7}{*}{ Zinc naphthenate } & & Ethanol & $\begin{array}{l}{[106] ; \quad[169] ;} \\
{[170]}\end{array}$ \\
\hline & & & Toluene & [52]; [468] \\
\hline & & & toluene: acetonitrile $=4: 1$ & {$[171]$} \\
\hline & & & toluene: acetonitrile $=8: 1$ & {$[466][467]$} \\
\hline & & & Toluene:methanol=7:3 & {$[172]$} \\
\hline & & & Methanol:acetic acid=1:1 & {$[80]$} \\
\hline & & & mineral spirits/xylene & [427] \\
\hline & \multirow[t]{3}{*}{ Zinc nitrate hexahydrate } & 36 & aqueous nitric acid solution & [154] \\
\hline & & & Ethanol & {$[81]$} \\
\hline & & & Water & [81];[142];[469] \\
\hline & $\begin{array}{l}\text { Zinc } \\
\text { tetramethyl-heptanedion } \\
\text { ate }\end{array}$ & & - & {$[174]$} \\
\hline \multirow[t]{4}{*}{$\mathrm{Ga}$} & $\begin{array}{lll}\begin{array}{l}\text { Gallium } \\
\text { hydrate }\end{array} & & \text { nitrate } \\
\end{array}$ & 110 & Ethanol & {$[161]$} \\
\hline & & & Water & [338] \\
\hline & $\begin{array}{l}\text { Gallium } \\
\text { tetramethyl-heptanedion } \\
\text { ate }\end{array}$ & $219-220$ & - & [174] \\
\hline & Trimethyl gallium & -15.8 & - & {$[176]$} \\
\hline $\mathrm{Ge}$ & & & & {$[177]$} \\
\hline
\end{tabular}




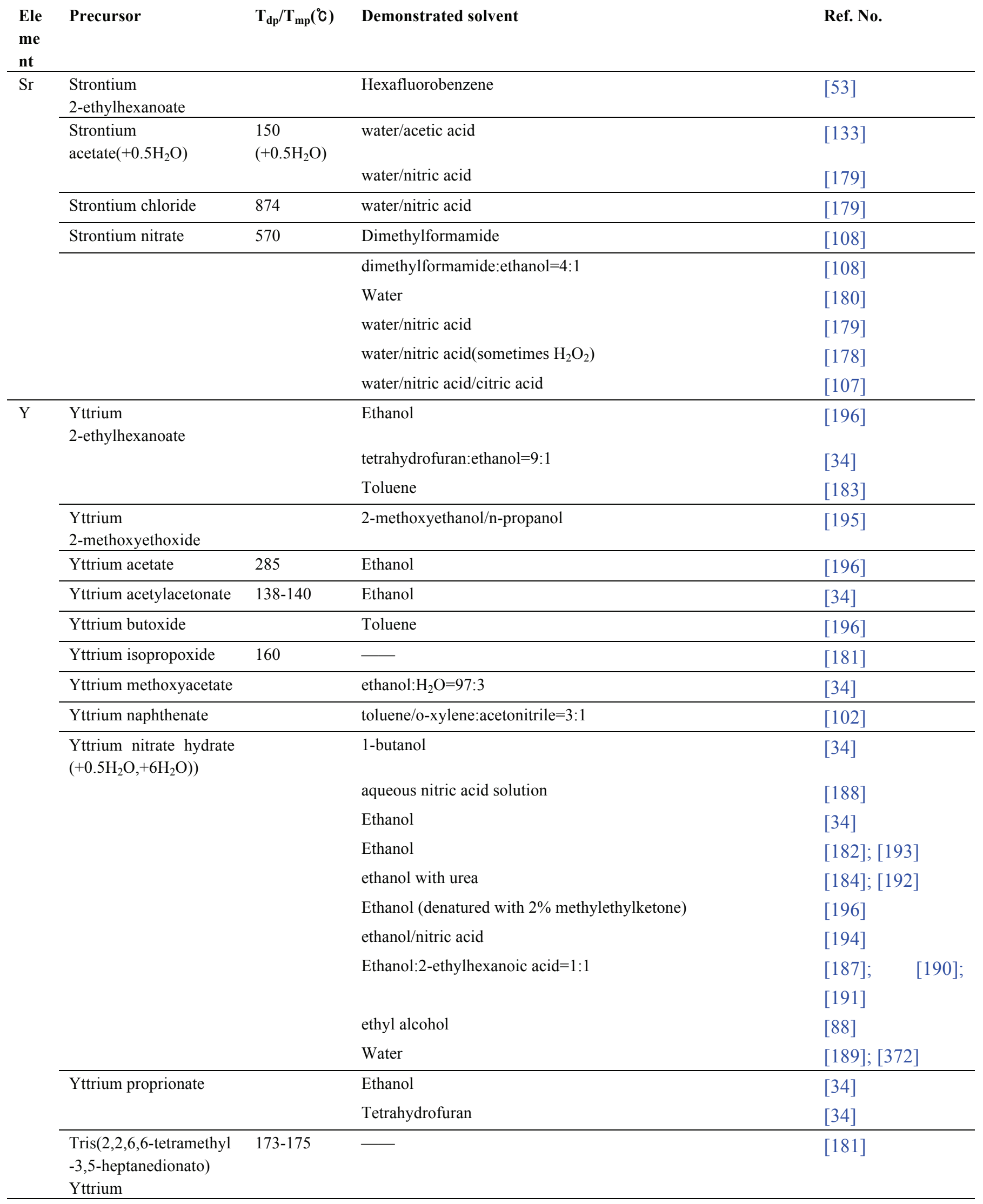




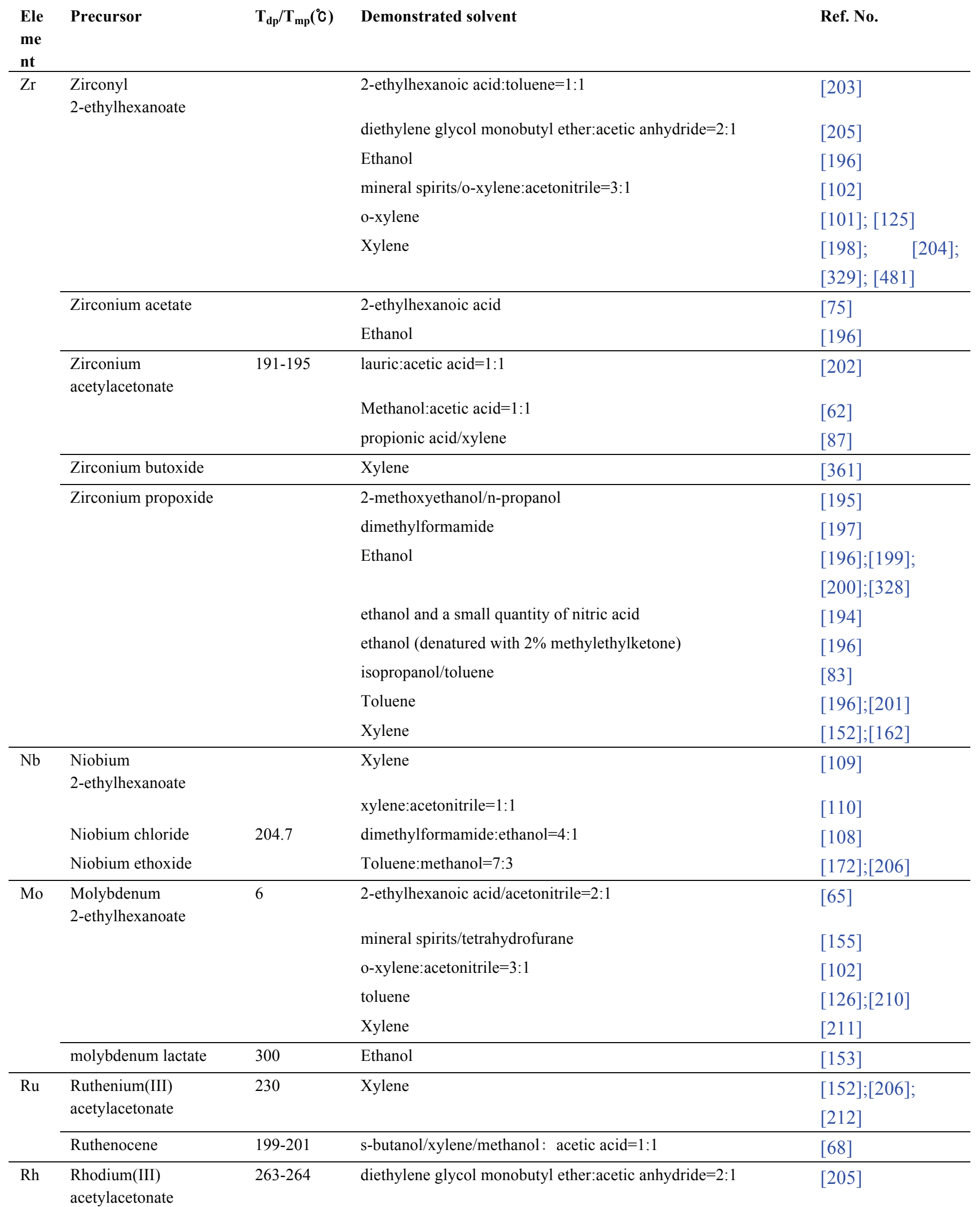




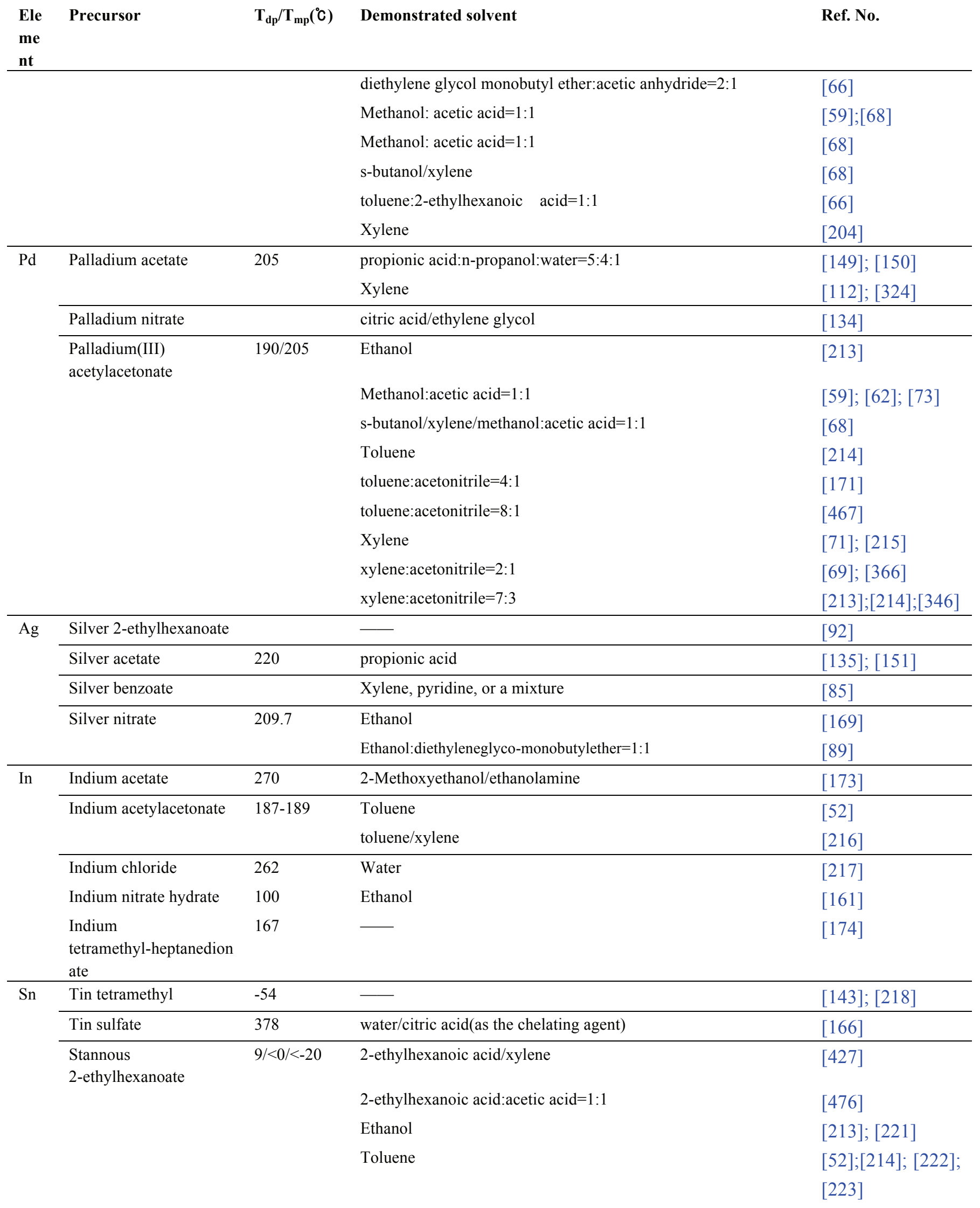




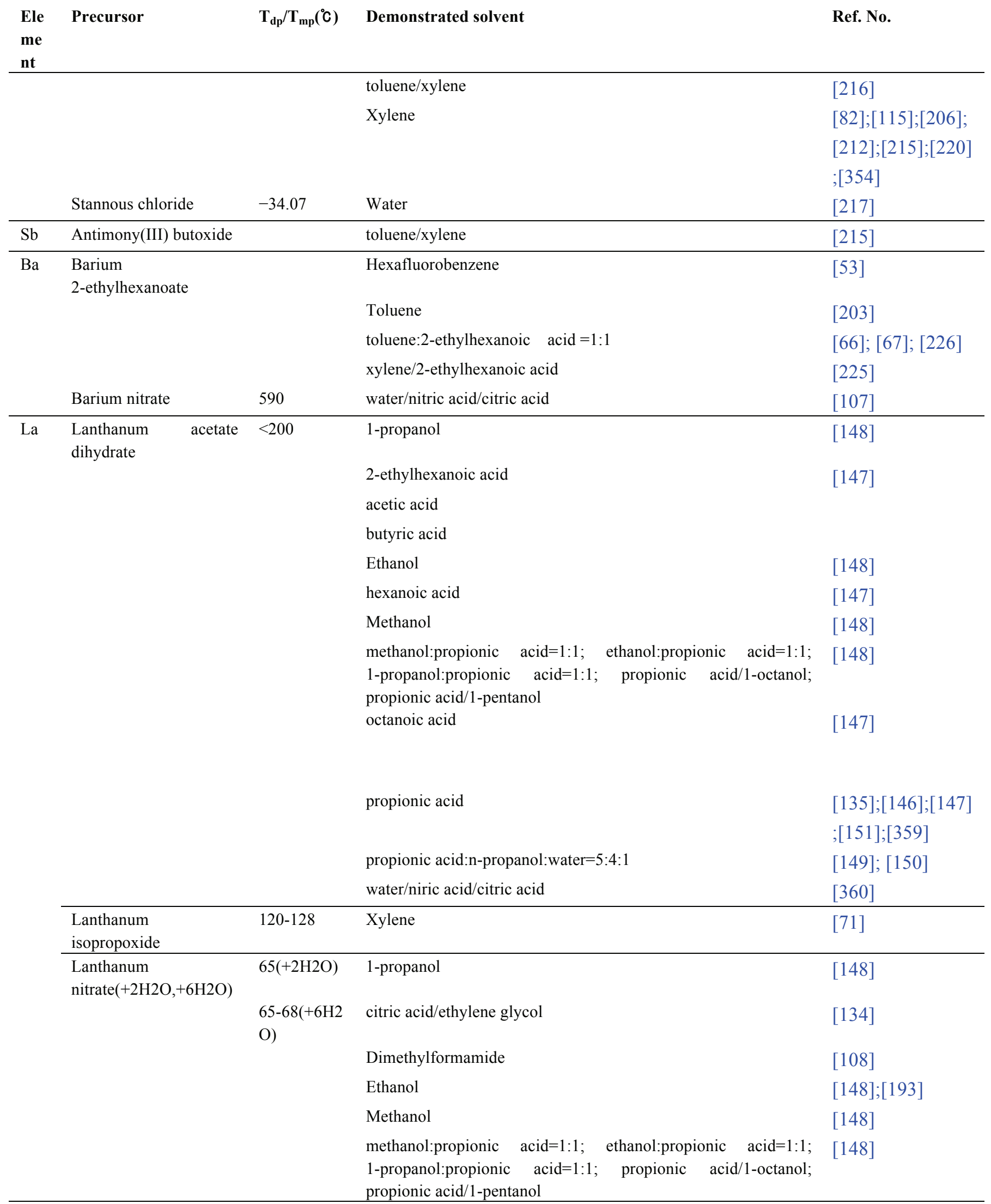




\begin{tabular}{|c|c|c|c|c|}
\hline $\begin{array}{l}\text { Ele } \\
\text { me } \\
\text { nt }\end{array}$ & Precursor & $\mathbf{T}_{\mathrm{dp}} / \mathbf{T}_{\mathrm{mp}}\left({ }^{\circ} \mathrm{C}\right)$ & Demonstrated solvent & Ref. No. \\
\hline \multirow[t]{23}{*}{$\mathrm{Ce}$} & Ceria(III) octoate & & Tetrahydrofuran & [228] \\
\hline & & & Toluene & [201] \\
\hline & cerium(III) & $>300$ & 2-ethylhexanoic acid/2-ethylhexanoic acid:toluene=1:1 & [203] \\
\hline & 2-ethylhexanoate & & & \\
\hline & & & 2-ethylhexanoic acid/o-xylene & {$[101]$} \\
\hline & & & 2-ethylhexanoic acid/xylene & [163]; [164] \\
\hline & & & diethylene glycol monobutyl ether:2-ethylhexanoic acid=1:1 & [89] \\
\hline & & & toluene: 2 -ethyl hexanoic acid $=1: 1$ & [226] \\
\hline & & & Xylene & {$[162] ;[204] ;[205]$} \\
\hline & $\begin{array}{l}\text { Cerium(III) acetate } \\
\text { hydrate }\end{array}$ & & 2-ethylhexanoic acid & {$[75]$} \\
\hline & {$\left[\mathrm{Ce}\left(\mathrm{CH}_{3} \mathrm{CO}_{2}\right)_{3}: \mathrm{H}_{2} \mathrm{O}\right]$} & & 2-ethylhexanoic acid/toluene & {$[75]$} \\
\hline & & & acetic acid & {$[42]$} \\
\hline & & & acetic acid/iso-octane:2-butanol $=4: 1$ & \\
\hline & & & lauric:acetic acid=1:1 & [202] \\
\hline & & & propanoic acid & [359] \\
\hline & $\begin{array}{l}\text { Cerium(III) } \\
\text { nitrate(+6H2O) }\end{array}$ & $\begin{array}{l}150 / 96(+6 \mathrm{H} \\
2 \mathrm{O})\end{array}$ & ethanol & {$[144]$} \\
\hline & & & ethanol & {$[74]$} \\
\hline & & & ethanol with urea & [192] \\
\hline & & & methanol & [230] \\
\hline & & & Water & [372] \\
\hline & & & water/niric acid/citric acid & [360] \\
\hline & Cerium(III) propionate & 120 & propionic acid & {$[12]$} \\
\hline & $\begin{array}{l}\text { Ammonium cerium(IV) } \\
\text { nitrate }\end{array}$ & $107-108$ & ethanol & {$[117]$} \\
\hline \multirow[t]{3}{*}{$\operatorname{Pr}$} & $\begin{array}{l}\text { Praseodymium } \\
\text { nitrate }\left(+2.7 \mathrm{H}_{2} \mathrm{O}, 6 \mathrm{H}_{2} \mathrm{O}\right)\end{array}$ & $56\left(+6 \mathrm{H}_{2} \mathrm{O}\right)$ & ethanol & {$[74]$} \\
\hline & & & water/nitric acid & [179] \\
\hline & & & water/nitric acid(sometimes $\mathrm{H}_{2} \mathrm{O}_{2}$ ) & [178] \\
\hline $\mathrm{Nd}$ & Neodymium nitrate & & ethanol & {$[141]$} \\
\hline \multirow[t]{6}{*}{$\mathrm{Eu}$} & $\begin{array}{ll}\begin{array}{l}\text { Colloidal } \\
\text { nanoparticles }\end{array} & \mathrm{Eu}_{2} \mathrm{O}_{3} \\
\end{array}$ & 2291 & Water & [180]; [231] \\
\hline & $\begin{array}{l}\text { Europium } \\
\text { 2-ethylhexanoate }\end{array}$ & & toluene & [183] \\
\hline & Europium(II) acetate & & water/niric acid/citric acid & [360] \\
\hline & $\begin{array}{l}\text { Europium } \\
\text { nitrate }\left(+6 \mathrm{H}_{2} \mathrm{O}\right)\end{array}$ & $85\left(+6 \mathrm{H}_{2} \mathrm{O}\right)$ & aqueous nitric acid solution & {$[188]$} \\
\hline & & & ethanol & {$[182]$} \\
\hline & & & ethanol with urea & [184] \\
\hline
\end{tabular}




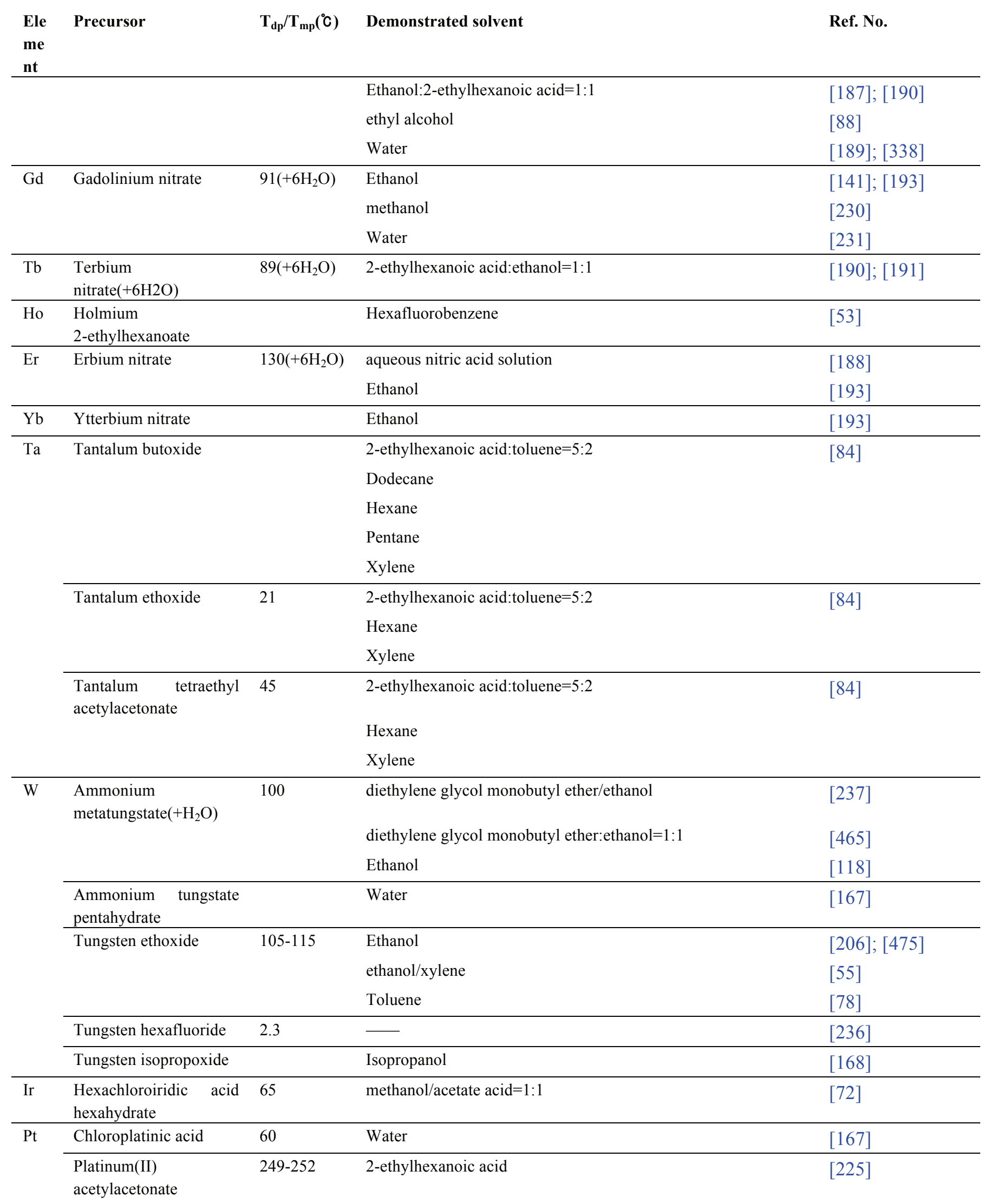




\begin{tabular}{|c|c|c|c|c|}
\hline \multirow[t]{15}{*}{$\begin{array}{l}\text { Ele } \\
\text { me } \\
\text { nt }\end{array}$} & Precursor & $\mathbf{T}_{\mathrm{dp}} / \mathbf{T}_{\mathrm{mp}}\left({ }^{\circ} \mathrm{C}\right)$ & Demonstrated solvent & Ref. No. \\
\hline & & & 2-ethylhexanoic acid:acetic acid=1:1 & {$[476]$} \\
\hline & & & diethylene glycol monobutyl ether:acetic anhydride $=2: 1$ & {$[67]$} \\
\hline & & & Ethanol & {$[206][475]$} \\
\hline & & & Methanol:acetic acid $=1: 1$ & {$[58] ;[59]$} \\
\hline & & & methanol:acetic acid=1:1 & {$[68]$} \\
\hline & & & Toluene & [222]; [223] \\
\hline & & & toluene:2-ethylhexanoic acid $=1: 1$ & {$[67] ;[226]$} \\
\hline & & & Xylene & {$[68] ;[206] ;[351][$} \\
\hline & & & & $354]$ \\
\hline & & & xylene/acetonitrile & {$[113] ;[114]$} \\
\hline & & & xylene/ethylacetate $=65: 35$ & {$[70]$} \\
\hline & & & Xylene:acetonitrile $=11: 5$ & {$[97] ;[119]$} \\
\hline & & & Xylene: acetonitrile $=2: 1$ & {$[69] ;[366]$} \\
\hline & & & Xylene: acetonitrile $=7: 3$ & {$[170]$} \\
\hline \multirow[t]{4}{*}{$\mathrm{Au}$} & $\begin{array}{l}\text { Dimethyl gold(III) } \\
\text { acetylacetonate }\end{array}$ & $81-82$ & xylene/pyridine & {$[85]$} \\
\hline & & & Xylene & {$[365]$} \\
\hline & $\begin{array}{l}\text { Gold(III) chloride } \\
\text { trihydrat }\end{array}$ & & Ethanol & {$[170]$} \\
\hline & $\begin{array}{l}\text { Gold-triphenylphosphin } \\
\text {-nitrate }\end{array}$ & & Tetrahydrofurane: isooctane $=1: 4$ & {$[350]$} \\
\hline $\mathrm{Pb}$ & $\begin{array}{l}\text { Lead(II) } \\
\text { 2-ethylhexanoate }\end{array}$ & & 2-ethylhexanoic acid:tetrahydrofuran $=2: 1$ & {$[120]$} \\
\hline \multirow[t]{8}{*}{$\mathrm{Bi}$} & $\begin{array}{l}\text { Bismuth(III) } \\
\text { 2-ethylhexanoate }\end{array}$ & & mineral spirits/tetrahydrofuran & {$[228]$} \\
\hline & & & Mineralspirits/toluene:2-ethylhexanoic acid=1:1 & {$[90]$} \\
\hline & & & Xylene & [211] \\
\hline & Bismuth acetate & 250 & 2-ethylhexanoic acid/xylene & {$[124]$} \\
\hline & $\begin{array}{l}\text { Bismuth nitrate } \\
\text { pentahydrate }\end{array}$ & 30 & acetic acid & {$[43]$} \\
\hline & & & ethanol & {$[123]$} \\
\hline & & & ethanol/water/nitric acid & {$[43]$} \\
\hline & & & nitric acid/water/denaturized absolute ethanol & [239] \\
\hline
\end{tabular}


Appendix Table 2. Properties of solvents used for flame spray pyrolysis of oxide nanomaterials.

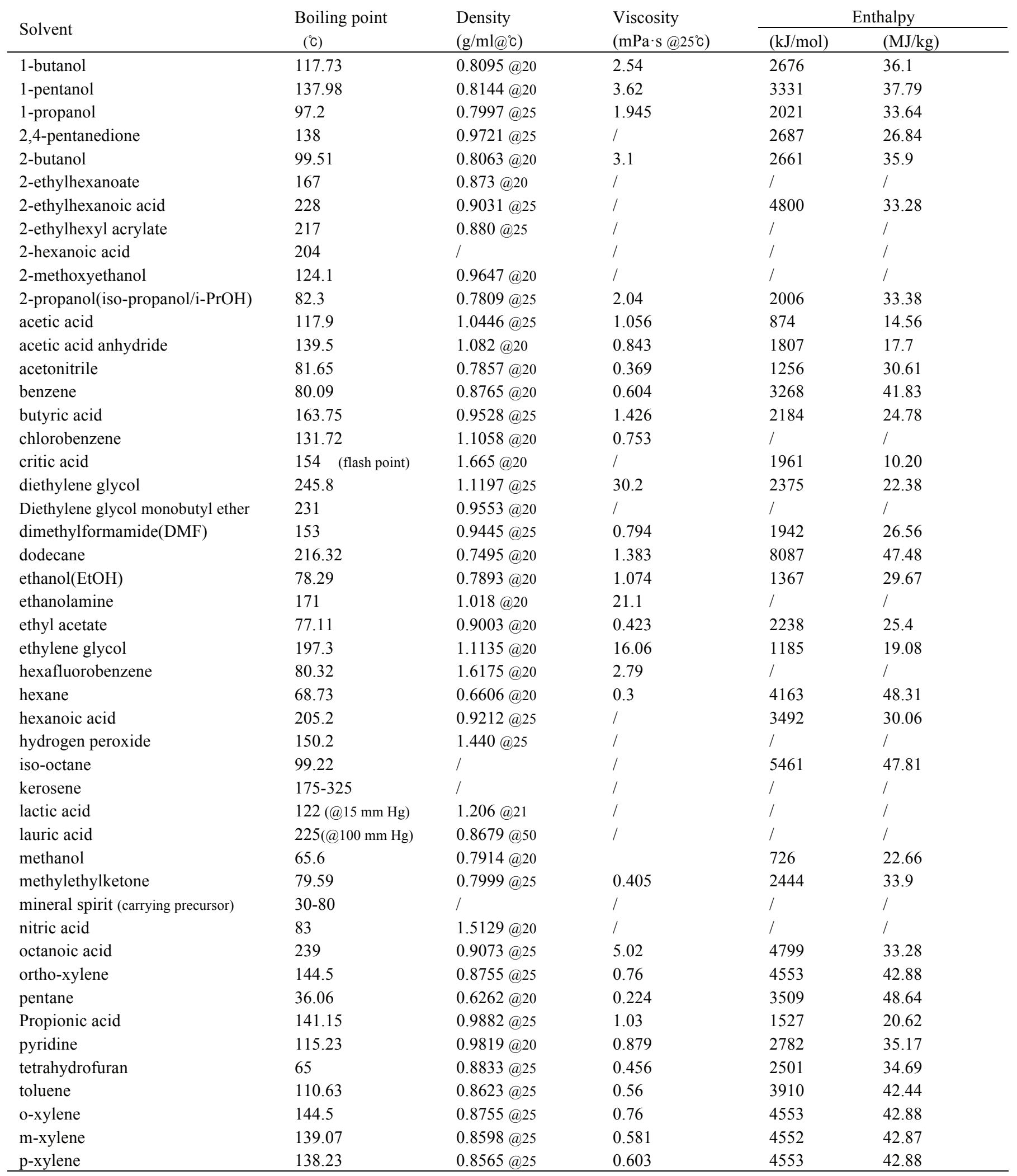


Tables

Table 1. Charateristic of various typical burner setups used in flame aerosol synthesis.

\begin{tabular}{|c|c|c|c|c|c|c|}
\hline $\begin{array}{l}\text { Burner } \\
\text { type }\end{array}$ & Mixing state & $\begin{array}{l}\text { Quenc } \\
\text { hing } \\
\text { rate }\end{array}$ & Application & Yield of NPs & Size of primary particle & Ref \\
\hline \multirow{2}{*}{$\begin{array}{l}\text { Coflow } \\
\text { burner }\end{array}$} & \multirow[t]{2}{*}{ Non-premixed } & \multirow{2}{*}{$\begin{array}{l}<100 \\
\mathrm{~K} / \mathrm{cm}\end{array}$} & \multirow{2}{*}{$\begin{array}{l}\text { both } \\
\text { lab-scale } \\
\text { and } \\
\text { industrial } \\
\text { units }\end{array}$} & $\begin{array}{l}\mathbf{T i O}_{2} \\
3.7 \mathrm{~g} / \mathrm{MJ} \text {-fuel } \\
920 \mathrm{~g} / \mathrm{min} \cdot \mathrm{m}^{2}\end{array}$ & $\begin{array}{l}11 \mathrm{~nm} \\
\text { (Inverse mixing) } \\
105 \mathrm{~nm} \\
\text { (Regular mixing) }\end{array}$ & $\begin{array}{l}{[255]} \\
{[256]}\end{array}$ \\
\hline & & & & $\begin{array}{l}\mathrm{SiO}_{2} \\
4.0-20 \mathrm{~g} / \mathrm{MJ}-\text { fuel } \\
980-3120 \mathrm{~g} / \mathrm{min} \cdot \mathrm{m}^{2}\end{array}$ & $\begin{array}{l}13-27 \mathrm{~nm}(\Delta v=20 \mathrm{~m} / \mathrm{s}) \\
57-86 \mathrm{~nm}(\Delta v=2 \mathrm{~m} / \mathrm{s})\end{array}$ & {$[257]$} \\
\hline \multirow{4}{*}{$\begin{array}{l}\text { McKenna } \\
\text { flat-flame } \\
\text { burner }\end{array}$} & \multirow{4}{*}{$\begin{array}{l}\text { Usually } \\
\text { premixed }\end{array}$} & \multirow{4}{*}{$\begin{array}{l}<100 \\
\mathrm{~K} / \mathrm{cm}\end{array}$} & \multirow{4}{*}{$\begin{array}{l}\text { limited to } \\
\text { lab-scale }\end{array}$} & $\begin{array}{l}\mathbf{T i O}_{\mathbf{2}}-\mathbf{S i O}_{\mathbf{2}} \\
0.4 \mathrm{~g} / \mathrm{MJ} \text {-fuel } \\
8.3 \mathrm{~g} / \mathrm{min} \cdot \mathrm{m}^{2}\end{array}$ & $\sim 15 \mathrm{~nm}$ & {$[270]$} \\
\hline & & & & $\begin{array}{l}\mathbf{S i O}_{2} \\
14.7 \mathrm{~g} / \mathrm{MJ}-\text { fuel } \\
475 \mathrm{~g} / \mathrm{min} \cdot \mathrm{m}^{2}\end{array}$ & $11 \mathrm{~nm}$ & {$[276]$} \\
\hline & & & & $\begin{array}{l}\mathbf{T i O}_{2} \\
4-15 \mathrm{~g} / \mathrm{MJ}-\text { fuel } \\
212-795 \mathrm{~g} / \mathrm{min} \cdot \mathrm{m}^{2}\end{array}$ & $25-45 \mathrm{~nm}$ & {$[277]$} \\
\hline & & & & $\begin{array}{l}\mathrm{TiO}_{2} \\
3.24 \mathrm{~g} / \mathrm{MJ}-\text { fuel } \\
105 \mathrm{~g} / \mathrm{min} \cdot \mathrm{m}^{2} \\
\end{array}$ & $39 \mathrm{~nm}$ & {$[278]$} \\
\hline \multirow{3}{*}{$\begin{array}{l}\text { Hencken } \\
\text { flat-flame } \\
\text { burner }\end{array}$} & \multirow{3}{*}{$\begin{array}{l}\text { Multi } \\
\text { non-premixed }\end{array}$} & \multirow{3}{*}{$\begin{array}{l}<100 \\
\mathrm{~K} / \mathrm{cm}\end{array}$} & \multirow{3}{*}{$\begin{array}{l}\text { Lab-sale, } \\
\text { but broader } \\
\text { flammabilit } \\
\text { y than } \\
\text { McKenna } \\
\text { burner }\end{array}$} & $\begin{array}{l}\mathrm{SiO}_{2} \\
3.8-5.3 \mathrm{~g} / \mathrm{MJ}-\text { fuel } \\
112-376 \mathrm{~g} / \mathrm{min} \cdot \mathrm{m}^{2} \\
\end{array}$ & $12.5-14.6 \mathrm{~nm}$ & {$[282]$} \\
\hline & & & & $\begin{array}{l}\mathbf{S n O}_{2} \\
0.83 \mathrm{~g} / \mathrm{MJ}-\text { fuel } \\
43.5 \mathrm{~g} / \mathrm{min} \cdot \mathrm{m}^{2} \\
\end{array}$ & $\begin{array}{l}12 \mathrm{~nm} \\
46 \mathrm{~nm} \text { (methane- } \\
\text { assisted) }\end{array}$ & $\begin{array}{l}{[287]-[2} \\
90]\end{array}$ \\
\hline & & & & $\begin{array}{l}\mathbf{T i O}_{2}, \mathbf{V}_{\mathbf{2}} \mathbf{O}_{\mathbf{5}}-\mathbf{T i O}_{\mathbf{2}} \\
0.01-0.013 \\
\mathrm{~g} / \mathrm{MJ} \text {-fuel } \\
0.12-0.15 \mathrm{~g} / \mathrm{min} \cdot \mathrm{m}^{2}\end{array}$ & $10-20 \mathrm{~nm}$ & $\begin{array}{l}{[295][29} \\
7][298]\end{array}$ \\
\hline \multirow[t]{2}{*}{$\begin{array}{l}\text { Counterflo } \\
\text { w burner }\end{array}$} & \multirow[t]{2}{*}{$\begin{array}{l}\text { Usually } \\
\text { non-premixed }\end{array}$} & \multirow[t]{2}{*}{$\begin{array}{l}\sim 4500 \\
\mathrm{~K} / \mathrm{cm}\end{array}$} & \multirow[t]{2}{*}{$\begin{array}{l}\text { limited to } \\
\text { lab-scale }\end{array}$} & $\begin{array}{l}\mathbf{A l}_{\mathbf{2}} \mathbf{O}_{3} \\
0.05-0.4 \mathrm{~g} / \mathrm{MJ}-\text { fuel } \\
2.0-17 \mathrm{~g} / \mathrm{min} \cdot \mathrm{m}^{2}\end{array}$ & $13-47 \mathrm{~nm}$ & {$[21]$} \\
\hline & & & & $\mathrm{SiO}_{2}:$ & $30-150 \mathrm{~nm}$ & {$[299][30$} \\
\hline
\end{tabular}




\begin{tabular}{|c|c|c|c|c|c|c|}
\hline & & & & $\begin{array}{l}80.8 \mathrm{~g} / \mathrm{MJ}-\text { fuel } \\
7.3 \mathrm{~g} / \mathrm{min} \cdot \mathrm{m}^{2}\end{array}$ & & 0] \\
\hline \multirow{2}{*}{$\begin{array}{l}\text { Premixed } \\
\text { stagnation } \\
\text { burner }\end{array}$} & \multirow{2}{*}{ Only premixed } & \multirow{2}{*}{$\begin{array}{l}>10^{4} \\
\mathrm{~K} / \mathrm{cm}\end{array}$} & \multirow{2}{*}{$\begin{array}{l}\text { currently } \\
\text { lab-scale }\end{array}$} & $\begin{array}{l}\mathbf{T i O}_{2}: \\
0.15-0.6 \quad \mathrm{~g} / \mathrm{MJ}-\text { fuel } \\
50-200 \mathrm{~g} / \mathrm{min} \cdot \mathrm{m}^{2}\end{array}$ & usually $<10 \mathrm{~nm}$ & $\begin{array}{l}{[10][96]} \\
{[112]}\end{array}$ \\
\hline & & & & $\begin{array}{l}\mathbf{T i O}_{2}: \\
0.45-2.41 \mathrm{~g} / \mathrm{MJ}-\text { fuel } \\
641-3436 \mathrm{~g} / \mathrm{min} \cdot \mathrm{m}^{2} \\
\end{array}$ & $7.5-17 \mathrm{~nm}$ & [318] \\
\hline \multirow{2}{*}{$\begin{array}{l}\text { FSP } \\
\text { burner }\end{array}$} & \multirow{2}{*}{$\begin{array}{l}\text { Non-premixed, } \\
\text { liquid-fed }\end{array}$} & \multirow{2}{*}{$\begin{array}{l}\sim 170 \\
\mathrm{~K} / \mathrm{cm}\end{array}$} & \multirow{2}{*}{$\begin{array}{l}\text { easily } \\
\text { scalable }\end{array}$} & $\begin{array}{l}\mathbf{Z r O}_{2}: \\
4.0 \mathrm{~g} / \mathrm{MJ} \text {-fuel }\end{array}$ & $20-100 \mathrm{~nm}$ & [329] \\
\hline & & & & $\begin{array}{l}\mathbf{S i O}_{2}: \\
23.6 \mathrm{~g} / \mathrm{MJ}-\text { fuel }\end{array}$ & $15-30 \mathrm{~nm}$ & [327] \\
\hline
\end{tabular}

Table 2. Laser diagnostic methods for gaseous flame fields during flame synthesis.

\begin{tabular}{|c|c|c|}
\hline Diagnostic methods & Variables & Reference and Contents \\
\hline $\begin{array}{l}\text { Spontaneous Raman } \\
\text { Scattering } \\
\text { (SRS) }\end{array}$ & Temperature & $\begin{array}{l}\mathrm{TiO}_{2} \text { synthesis. Zhang et al., Combust. \& Flame, 2013. [493] } \\
\mathrm{TiO}_{2} \text { synthesis. Liu et al., Appl. Phys. B., 2010. [492] } \\
\mathrm{SiO}_{2} \text { synthesis. Allendorf et al., J. Appl. Phys., 1989. [491] }\end{array}$ \\
\hline $\begin{array}{l}\text { Coherent anti-Stokes } \\
\text { Raman Scattering } \\
\text { (CARS) }\end{array}$ & Temperature & $\begin{array}{l}\text { Diamond synthesis. Bertagnolli \& Lucht. } 26^{\text {th }} \text { Symposium on } \\
\text { Combustion, 1996. [315] } \\
\mathrm{TiO}_{2} \text { synthesis. Kammler et al., Combust. \& Flame, 2002. [278] } \\
\mathrm{SiO}_{2} \text { synthesis. Engel et al., Appl. Optics, 2012. [495] } \\
\mathrm{SiO}_{2} \text { synthesis. Hwang et al., J. Aerosol Sci., 2001. [496] } \\
\mathrm{SiO}_{2} \text { synthesis. Kim et al., Int. J. Heat Mass Transf., 2005. [497] }\end{array}$ \\
\hline $\begin{array}{l}\text { Fourier Transform infrared } \\
\text { emission/transmission } \\
\text { Spectroscopy } \\
\text { (FTIR) }\end{array}$ & Temperature & $\begin{array}{l}\mathrm{SiO}_{2} \text { synthesis. Mädler, et al., J. Aerosol Sci., 2002. [41] } \\
\mathrm{ZrO}_{2} \text { synthesis. Gröhn et al., Chem. Eng. J., 2012. [328] } \\
\mathbf{P t} / \mathbf{T i O}_{2} \text { synthesis. Schulz et al., J. Mater. Res., 2005. [500] } \\
\mathbf{F e}_{2} \mathbf{O}_{3} \text { synthesis. Ruding \& Pratsinis, Ind. Eng. Chem. Res., } 2012 . \\
{[501]}\end{array}$ \\
\hline $\begin{array}{l}\text { Laser-induced } \\
\text { Fluorescence } \\
\text { (LIF) }\end{array}$ & $\begin{array}{l}\text { Radicals } \\
\text { concentrations }\end{array}$ & $\begin{array}{l}\text { OH in } \mathrm{SiO}_{2} \text { synthesis. Hwang et al., J. Aerosol Sci., 2001. [496] } \\
\text { OH multilines in } \mathrm{SiO}_{2} \text { synthesis. Glumac et al., J. Mater. Res., } \\
\text { 1997. [502] }\end{array}$ \\
\hline & Temperature & $\begin{array}{l}\text { NO multilines in } \mathrm{TiO}_{2} \text { synthesis. Kronemayer et al., Appl. Phys. B, } \\
\text { 2007. [504] } \\
\text { NO multilines in } \mathrm{SiO}_{2} \text { synthesis. Hecht et al., Appl. Phys. B, } 2008 \text {. } \\
\text { [505] }\end{array}$ \\
\hline PIV/LPD & Velocity & $\begin{array}{l}\mathrm{ZrO}_{2} \text { synthesis. Gröhn \& Pratsini, J. Chem. Eng. 2012. [328] } \\
\mathrm{SiO}_{2} \text { synthesis. Camenzind et al., Eur. J. Inorg. Chem., 2008. [525] }\end{array}$ \\
\hline
\end{tabular}


Table 3. Excitation wavelength for laser-induced fluorescence of intermediates in precursor reactions.

\begin{tabular}{lll}
\hline Species & Excitation laser wavelength & Reference \\
\hline $\mathrm{Si}$ & $251.61 \mathrm{~nm}, 3 p^{3} P_{2}-4 s^{3} P_{2}$ & Muramoto et al., Appl. Phys. Lett., 2000. [511] \\
$\mathrm{SiO}$ & $231 \mathrm{~nm}, \quad A-X(1-0)$ & Glumac. Combust. \& Flame, 2001. [507] \\
$\mathrm{Fe}$ & $225.15 \mathrm{~nm}, a^{5} D^{-} F^{0}$ & Hecht et al., Applied Physics B, 2009. [505] \\
$\mathrm{FeO}$ & $448 \mathrm{~nm}, \quad X^{5} \Delta_{4^{-}}{ }^{-} \Delta_{4}(0-0)$ & McMillin et al., J. Mater. Res., 1996. [508] \\
$\mathrm{PbO}$ & $567.5-571.5 \mathrm{~nm}, X^{l} \Sigma\left(0^{+}\right)-A^{3} \Pi\left(0^{+}\right)(0-3)$ & Biswas \& Zachariah, Environ. Sci. Technol. 1997. [509] \\
$\mathrm{TiO}$ & $496 \mathrm{~nm}, C^{3} \Delta-X^{3} \Delta(1-0)$ & Colibaba-Evulet et al., Combust. Sci. Technol., 2000. [510] \\
$\mathrm{SnO}$ & $226.89 \mathrm{~nm}, E^{l} \Sigma^{+}-X^{l} \Sigma^{+}(18-0)$ & Bailey \& Glumac, Appl. Phys. B., 2003. [506] \\
$\mathrm{AlO}$ & $465 \mathrm{~nm}, B^{2} \Sigma^{+}-X^{2} \Sigma^{+}(1-0)$ & Colibaba-Evulet et al., Combust. Sci. Technol., 2000. [510] \\
\hline
\end{tabular}

Table 4. Laser diagnostic methods for particles during flame synthesis.

\begin{tabular}{|c|c|c|}
\hline $\begin{array}{l}\text { Diagnostic } \\
\text { methods }\end{array}$ & Variables & Reference and Contents \\
\hline \multirow[t]{2}{*}{ LIS-Rayleigh } & $\begin{array}{l}\text { Size, } \\
\text { fractal number }\end{array}$ & $\begin{array}{l}\mathrm{TiO}_{2} \text { nanoparticles synthesis. Yang et al., Aerosol Sci.Technol. } 2007 \\
{[516]}\end{array}$ \\
\hline & Size & $\mathbf{A l}_{2} \mathbf{O}_{3}$ nanoparticles synthesis. Xing et al., Appl. Optics. 1999 [308] \\
\hline \multirow[t]{2}{*}{ LIS-SAXS } & Size & $\mathrm{SiO}_{2}$ nanoparticles synthesis. Beaucage et al., Nat. Mater. 2004 [524] \\
\hline & $\begin{array}{l}\text { Primary particle size, volume } \\
\text { fraction, aggregate } \\
\text { radius, mass fration } \\
\text { dimension }\end{array}$ & $\begin{array}{l}\mathrm{SiO}_{2} \text { nanoparticles synthesis. Camenzind et al., Eur. J. Inorg. Chem. } \\
2008 \text { [525] }\end{array}$ \\
\hline LIS-DLS & Size & $\begin{array}{l}\mathrm{SiO}_{2} \text { nanoparticles synthesis. Zachariah et al., Applied optics, } \\
1989[526]\end{array}$ \\
\hline \multirow[t]{2}{*}{ LIS-PDA } & Size & $\begin{array}{l}\mathrm{ZrO}_{2} \text { nanoparticles synthesis. Heine et al., Ind. Eng. Chem. Res. } \\
2005[527]\end{array}$ \\
\hline & Droplet size, Particle size & $\mathrm{ZrO}_{2}$ nanoparticles synthesis. Gröhn et al., Chem. Eng. J. 2012[328] \\
\hline Raman & Crystallinity & $\begin{array}{l}\mathrm{TiO}_{2} \text { nanoparticles synthesis. Liu et al., Applied Physics B. } 2010 \\
\text { [492] }\end{array}$ \\
\hline \multirow[t]{5}{*}{ LII } & Volume fraction & Soot formation. Shaddix et al., Combust. Flame. 1996 [529] \\
\hline & Size & $\begin{array}{l}\mathrm{TiO}_{2} \text { nanoparticles synthesis. Cignoli, et al., Applied Physics B, } \\
2009[538]\end{array}$ \\
\hline & Size & $\begin{array}{l}\text { Gas-to-particle converstion of } \mathbf{T i O}_{2} \text {. Lehre, et al., Proc. Combust. } \\
\text { Inst. } 2005 \text { [537] }\end{array}$ \\
\hline & Size & $\begin{array}{l}\mathrm{TiO}_{2} \text { nanoparticles synthesis. Maffi, et al., Spectrochimica Acta Part } \\
\text { B: Atomic Spectroscopy, } 2008 \text { [541] }\end{array}$ \\
\hline & Size & Si nanoparticles plasma synthesis. Sipkens et al., Appl. Phys. B, \\
\hline
\end{tabular}




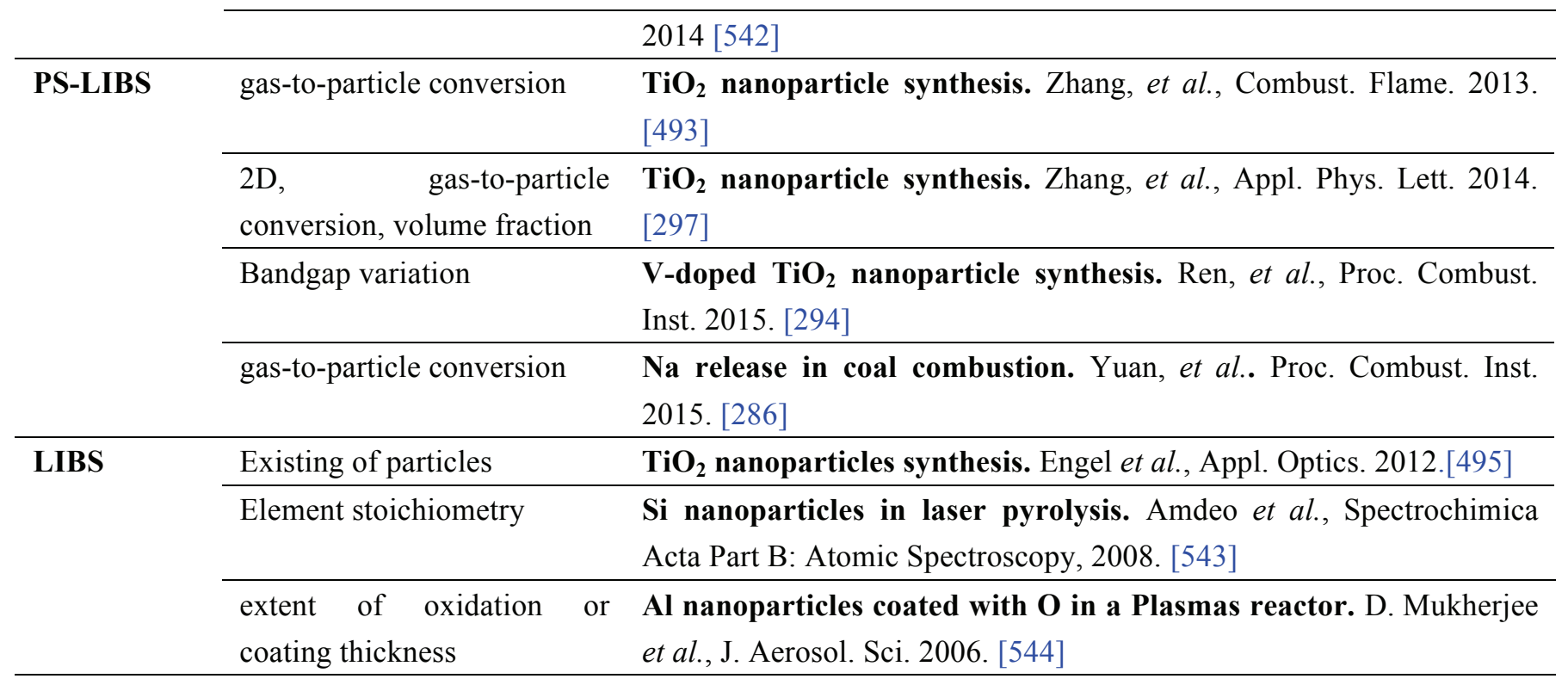

Table 5. Summary of chemical kinetics of precursors.

\begin{tabular}{|c|c|c|c|c|}
\hline Element & Reaction & $\mathrm{k}\left(\mathrm{s}^{-1}\right)$ & $\mathrm{E}(\mathrm{kJ} / \mathrm{mol})$ & Remark \\
\hline \multirow{6}{*}{$\mathrm{Ti}$} & $\mathrm{TTIP} \rightarrow \mathrm{TiO}_{2}+4 \mathrm{C}_{3} \mathrm{H}_{6}+2 \mathrm{H}_{2} \mathrm{O}$ & $3.96 \times 10^{5}$ & 70.5 & $\begin{array}{l}\text { Okuyama et al., AIChE J. } \\
\text { 1990. [569] }\end{array}$ \\
\hline & $\mathrm{TTIP}+2 \mathrm{H}_{2} \mathrm{O} \rightarrow \mathrm{TiO}_{2}+4 \mathrm{C}_{3} \mathrm{H}_{7} \mathrm{OH}$ & $3 \times 10^{15}$ & 8.4 & $\begin{array}{l}\text { Seto et al., Aerosol. Sci. Tech. } \\
\text { 1995. [573] }\end{array}$ \\
\hline & & & & Tsantilis et al., Chem. Eng. \\
\hline & TTIP $\stackrel{\text { surface }}{\longrightarrow}-\mathrm{FiO}_{2} \quad 4 \mathrm{C}_{3} \mathrm{H}_{6}+2 \mathrm{H}_{2} \mathrm{O}$ & $1 \times 10^{9} \mathrm{~A} \rho_{\mathrm{g}}$ & 126 & Sci. 2002. [275] \\
\hline & & & & Battiston et al., 1997. [574] \\
\hline & $\mathrm{TiCl}_{4}+\mathrm{O}_{2} \rightarrow \mathrm{TiO}_{2}+2 \mathrm{Cl}_{2}$ & $8 \times 10^{4} \mathrm{~s}^{-1}$ & 89 & $\begin{array}{l}\text { 973-1373K. Pratsinis, } 1999 . \\
{[270]}\end{array}$ \\
\hline \multirow{3}{*}{$\mathrm{Fe}$} & $\mathrm{Fe}\left(\mathrm{C}_{5} \mathrm{H}_{5}\right)_{2} \rightarrow \mathrm{Fe}+2 \mathrm{C}_{5} \mathrm{H}_{5}$ & $2.19 \times 10^{16}$ & 382 & $\begin{array}{l}\text { Kuwana et al., 2007, Proc. } \\
\text { Combust. Inst. [575] }\end{array}$ \\
\hline & $\mathrm{Fe}\left(\mathrm{C}_{5} \mathrm{H}_{5}\right)_{2} \rightarrow \mathrm{Fe}+\mathrm{H}_{2}+\mathrm{CH}_{4}+\mathrm{C}_{5} \mathrm{H}_{6}$ & $3 \times 10^{4}$ & 67 & $\begin{array}{l}\text { Bhattacharjee et al. J. } \\
\text { Exp.Phys. 2014. [576] }\end{array}$ \\
\hline & $\mathrm{Fe}\left(\mathrm{C}_{5} \mathrm{H}_{5}\right)_{2} \rightarrow \mathrm{Fe}+2 \mathrm{C}_{5} \mathrm{H}_{5}$ & $10^{10}$ & 171 & $\begin{array}{l}\text { Kuwana et al., 2007, Proc. } \\
\text { Combust. Inst. [575] }\end{array}$ \\
\hline V & $\mathrm{VO}(\text { acac })_{2}+\mathrm{O}_{2} \rightarrow \mathrm{V}_{2} \mathrm{O}_{5}+\mathrm{CO}_{2}+\mathrm{H}_{2} \mathrm{O}$ & $5 \times 10^{8}$ & 90.5 & $\begin{array}{l}\text { Galembeck \& Alves, J. Mater. } \\
\text { Sci., 2002. [577] }\end{array}$ \\
\hline $\mathrm{Si}$ & HMDS $\rightarrow$ Product & $4 \times 10^{17}$ & 370 & $\begin{array}{l}\text { Ehrman et al. J Aerosol Sci. } \\
\text { 1998. [270] }\end{array}$ \\
\hline
\end{tabular}




$\begin{array}{llll}\mathrm{SiCl}_{4}+\mathrm{O}_{2} \rightarrow \mathrm{SiO}_{2}+2 \mathrm{Cl}_{2} & 8 \times 10^{14} & 410 & \begin{array}{l}\text { Ehrman et al. J Aerosol Sci. } \\ 1998 \text { [270] }\end{array} \\ \mathrm{Si}\left(\mathrm{OC}_{2} \mathrm{H}_{5}\right)_{4}+2 \mathrm{H}_{2} \mathrm{O} \rightarrow \mathrm{SiO}_{2} \downarrow+4 \mathrm{C}_{2} \mathrm{H}_{5} \mathrm{OH} & 0.2625 & 22.05 & \begin{array}{l}\text { Zhao et al. Acta Physico- } \\ \text { Chimica Sinica 1995 [578] }\end{array}\end{array}$

Table 6. Summary of statistical values in MD simulation.

\begin{tabular}{|c|c|c|}
\hline Statistical value & Content & Expression \\
\hline Temperature & $\begin{array}{l}\text { Average } \\
\text { kinetic energy }\end{array}$ & $T=\frac{\sum_{i} m v_{i}^{2}}{k_{b} f}, f$ is the degree of freedom. \\
\hline Potential energy & Melting point & $\begin{array}{l}U=\sum_{i \neq j} U_{i j}, U_{i j} \text { is the potential energy between every two atom } i \\
\text { and } j\end{array}$ \\
\hline Lindemann index & Melting point & $\begin{array}{l}L=\frac{1}{N(N-1)} \sum_{i \neq j} \frac{\sqrt{\left\langle r_{i j}^{2}\right\rangle_{t}-\left\langle r_{i j}\right\rangle_{t}^{2}}}{\left\langle r_{i j}\right\rangle_{t}^{2}}, r_{i j} \text { is the distance between atom } i \\
\text { and } j,\langle\rangle_{t} \text { indicates an average over time. }\end{array}$ \\
\hline $\begin{array}{l}\text { Local lattice } \\
\text { orientation }\end{array}$ & $\begin{array}{l}\text { local } \\
\text { crystallization }\end{array}$ & $\begin{array}{l}\text { X component of the normal vector of plane constructed by three } \\
\text { neighboring Ti atoms around each } O \text { atom as detailed in Zhang et } \\
\text { al. [593] }\end{array}$ \\
\hline $\begin{array}{l}\text { Coordination } \\
\text { number }\end{array}$ & Crystallization & $\begin{array}{l}f_{i}=\sum_{r_{i j}<l} n_{i}, n_{i} \text { is the number of atoms around atom } i \text { within the } \\
\text { length of crystal lattice } l .\end{array}$ \\
\hline X-ray diffraction & Crystallization & $\begin{array}{l}I_{k}(b)=\sum_{\mathrm{i}, j, 1 \neq j} f_{i}(b) f_{j}(b) \frac{\sin \left(2 \pi b r_{i j}\right)}{2 \pi b r_{i j}}, b=2 \sin \theta / \lambda \text { where } 2 \theta \text { is the } \\
\text { scattering angle and } \lambda \text { is the wavelength of the incident radiation } \\
\text { taken to be } 0.15418 \mathrm{~nm}, r_{i j} \text { is the distance between atoms } i \text { and } j, \\
f_{i}(b) \text { and } f_{j}(b) \text { is the scattering factors for atoms } i \text { and } j .\end{array}$ \\
\hline $\begin{array}{l}\text { Distribution } \\
\text { function }\end{array}$ & $\begin{array}{l}\text { (Local) } \\
\text { Crystallization }\end{array}$ & $\begin{array}{l}g_{i j}(r)=\frac{d\left\langle n_{i j}\right\rangle / d r}{N_{j} 4 \pi r^{2}}, \text { where } r \text { is the radial distance, }\left\langle n_{i j}\right\rangle \text { is the } \\
\text { assemble average of the coordination number of atom } j \text { around atom } \\
i \text { in a spherical shell with a radius of } \mathrm{r} \text { and a thickness of } d r \text {. } \\
g(r)=\sum_{i, j} c_{i} c_{j} g_{i j}(r) \text { represents total radial-distribution function }\end{array}$ \\
\hline
\end{tabular}


where $c_{i}, c_{j}$ are the concentration of atom $i$ and $j$

\begin{tabular}{|c|c|c|}
\hline Surface area $\left(\mathrm{m}^{2}\right)$ & Sintering level & Meyer's method \\
\hline $\begin{array}{l}\text { Normalized } \\
\text { surface area }\end{array}$ & Sintering level & $\begin{array}{l}a^{*}=\left(a-a_{0}\right) /\left(a_{0}-a_{f}\right) \text {, where } a_{0} \text { is the initial surface, } a_{f} \text { is the initial } \\
\text { surface }\end{array}$ \\
\hline Gyration radius & Sintering level & $\begin{array}{l}R_{g}=\sqrt{\frac{1}{M} \sum_{i} m_{i}\left(r_{i}-r_{c}\right)^{2}}, M \text { is the total mass of particles and } r_{c} \text { is } \\
\text { the center-of-mass position of particles. }\end{array}$ \\
\hline $\begin{array}{l}\text { Ratio of moment } \\
\text { of inertia }\end{array}$ & Sintering level & $\begin{array}{l}R=I_{z} / I_{x y}, I_{z} \text { is the moment of inertial with respect to the } \mathrm{Z} \text { axis } \\
\text { which go through the centers of two particles while } I_{x y} \text { is the } \\
\text { moment of inertial with respect to the axis along the direction of } \\
\text { collision. }\end{array}$ \\
\hline Shrinkage & Sintering level & $\begin{array}{l}\text { shrinkage }=\frac{\left(d_{1}+d_{2}\right) / 2-d_{c o m}}{\left(d_{1}+d_{2}\right) / 2}, d_{1} \text { and } d_{2} \text { are the diameters of the } \\
\text { sintering particles and } d_{c o m} \text { is the distance between their centers of } \\
\text { mass at that distance }\end{array}$ \\
\hline $\begin{array}{l}\text { Mean square } \\
\text { displacement }\end{array}$ & Atom mobility & $\begin{array}{l}D=\lim _{t \rightarrow \infty} \frac{\left\langle r^{2}\right\rangle}{2 d t},\left\langle r^{2}\right\rangle \text { is the mean square displacement of atoms in } \\
\text { time } t \text { and } d \text { is the dimensionality of the system }\end{array}$ \\
\hline
\end{tabular}




\section{Figure Captions}

Fig. 1. Schematic of particle formation processes during vapor-fed or liquid-fed flame synthesis through mechanisms of gas-to-particle conversion, intra-particle reaction, etc.

Fig. 2. Schematics of various typical burner setups used in flame synthesis.

Fig. 3. The temperature, fluid flow and species mole fractions fields of classical 1-D stagnation premixed flames along the centerline of a typical premixed stagnation flame (Mixture: $3.96 \%-\mathrm{C}_{2} \mathrm{H}_{4} / 26.53 \%-\mathrm{O}_{2} / 69.51 \%$-Ar; Equivalence ratio: $\Phi=0.45$; Configuration: I.D. $1 \mathrm{~cm}$ nozzle with nozzle-substrate distance $3.4 \mathrm{~cm}$ ), as adapted from literature [311]. The steepest temperature gradient at the cold stagnation plate reaches as large as $5 \times 10^{4} \mathrm{~K} / \mathrm{cm}$.

Fig. 4. Techniques of various spraying atomizers used in flame spray pyrolysis. (A) Lab-scale two-phase atomizer, with liquid precursor delivered through the center capillary and dispersed with oxygen, along with its location in a cylindrical reactor chamber [329]; (B) Large-scale novel atomization combining a pressure swirl nozzle (liquid hollow cone nozzle) and a dispersion gas nozzle, where $\alpha$ is the gas inclination angle, $\beta$ is the gas swirl angle and $\gamma$ is the for liquid hollow cone angle [331]; (C) Lab-scale electrospray atomizer [195]. (Reprinted with permission from literature [195][329][331]).

Fig. 5. Flame-made nanocomposite materials of metal-support mixture with heterogeneously dispersed structures. A: $\mathrm{TiO}_{2}$ supported Pd nanoparticles made by Niu et al. JPCC, 2014 [112]; $\mathrm{B}: \mathrm{Al}_{2} \mathrm{O}_{3}$ supported Pt-Pd alloy particles made by Strobel et al., Catal. Lett. 2005 [69]; C: Pd nanoparticles on mixed $\mathrm{SiO}_{2}-\mathrm{Al}_{2} \mathrm{O}_{3}(\mathrm{Pd} / \mathrm{SA})$ with tunable surface acidity made by Huang et al., J. Catal. 2011 [73]; D: Pd-containing perovskite-type oxide catalyst made by Lu et al., Appl. Catal. B. 2014 [358]; E: Pt/Ba on $\mathrm{CeO}_{2}-\mathrm{ZrO}_{2}$ mixed support by Strobel et al. J. Catal. 2006 [358]. (Reprinted with permission from literature [112] [69] [73] [358] [203]). 
Fig. 6. Flame-made nanocomposite materials with core-shell structures. (A): Shell- $\left(\mathrm{Ce}_{0.7} \mathrm{Zr}_{0.3} \mathrm{O}_{2}\right) 0.5$ / Core- $\left(\mathrm{Al}_{2} \mathrm{O}_{3}\right) 0.5$ by Weidenhof et al., JACS, 2009, 131, p9207 [35]; (B): Shell-TiO 2 (12 atomic \%)/Core- $\mathrm{CeO}_{2}$ by Feng et al., Science, 2006 [12]; C: Shell-SiO $2 /$ Core- $\mathrm{Y}_{2} \mathrm{Si}_{2} \mathrm{O}_{7}:$ Eu, Hasegawa et al. Proc. Combust. Inst., 2011 [189]. (Reprinted with permission from literature [12][35][189]).

Fig. 7. Flame-made nanocomposite materials with homogenously-mixed single-phase structures. (A) low-resolution and high resolution TEM images of single particles, with magnified portion of the particles and respective SAED patterns of pure $\mathrm{TiO}_{2}$ (upper panel), 4 atom \% Fe-doped $\mathrm{TiO}_{2}$ (middle panel), and 10 atom \% Fe-doped $\mathrm{TiO}_{2}$ (lower panel); (B) Energy-filtered TEM (EFTEM) of 10 atom \% Fe-doped TiO2 nanoparticles. (Reprinted with permission from literature by George et al. JACS, 2011 [105]).

Fig. 8. Doping diagram of flame synthesis for achieving various hierarchical structures.

Fig. 9. Morphologies and dot-mapping images of dispersed Carbon-Mn3O4 composite structure prepared by flame spray pyrolysis: (a) TEM image, (b) high-resolution TEM image, and (c) dot-mapping images. (Reprinted with permission from Ko et al. Scientific Reports 2014 [375]).

Fig. 10. Morphologies and dot-mapping images of amorphous carbon-MnOx nanocomposite particles: (a) TEM and SAED images; (b) EDS mapping image of distribution of elemental carbon and Mn. (Reprinted with permission from Guo et al. Adv. Funct. Mater. 2012 [376]).

Fig. 11. Illustration of three deposition and film growth mechanisms (left part) and its controlling diagram as functions of particle diameter and size distribution function of particle number concentration. When the residence time is much smaller than the chemical reaction time, vapor deposits on the plate and forms the dense smooth film which corresponed to "chemical vapor deposition"; When the 
residence time is larger than the chemical reaction time, as-synthesized particles deposit on the plate and form the dense granular film which correspond to "aerosol deposition"; When the residence time is smaller or comparative to the chemical reaction time, mixture of vapor and particles deposits on the plate and forms the columnar film which corrpond to the mix deposition regime. (Reprinted from An et al. 2009, [428]).

Fig. 12. Specific surface area (SSA) of $\mathrm{TiO}_{2}$ nanofilms (granular-regime) under different substrate temperatures. Open circles represent the data for a substrate temperature of $383 \mathrm{~K}$, while the filled squares correspond to a substrate temperature of $763 \mathrm{~K}$. TEM images on the right-hand side show the morphologies of the nanofilms at (A) $116.4 \mathrm{ppm}$ and (B) $291.0 \mathrm{ppm}$, for a substrate temperature of 763 K. (Reprinted with permission from Zhang et al. 2012 [10]).

Fig. 13. Characterization of the laminar boundary layer above a stagnation plane for particle deposition by comparing the profiles between gas velocity and thermophoretic velocity. (The Renolds number for the stagnation jet is around 1500, the stagnation strain rate is around $60 \mathrm{~s}^{-1}$, and the tempartue of gas outside the boundary layer about $1700 \mathrm{~K}$ ) (Reprinted with permission from Zhang et al. 2012, [323]).

Fig. 14. The variation of the normalized deposition flux $\phi / c 1$ th obtained from Eqn. (5.7) and corresponding the thermophoretic Peclet number, Peth, as a function of nanoparticle diameter (Reprinted with permission from Zhang et al. 2012 [323]).

Fig. 15. Fabrication of TiO2 films by vapor-fed stagnation flame aerosol deposition for both $\mathrm{CO}$ gas sensing and dye-sensitized solar cell applications. The right (A) shows the stagnation flame aerosol deposition by a premixed stagnation flame seeded with TTIP (adapted from Wang and co-workers [318] with permissions); The middle top (B) shows the reponses to $\mathrm{CO}$ exposure at $773 \mathrm{~K}$ for sensor films (Adapted from Wang and co-workers [461] with permissions); The right top (C) shows photocurrent 
density-voltage characteristics of dye sensitized solar cells (Adapted from Wang and co-workers [462] with permissions); The bottom right shows the SEM images of mesoporous TiO2 films before and after densification and sintering (Adapted from Wang and co-workers [461] for the right part and [462] for the left partwith permissions).

Fig. 16. Fabrication of $\mathrm{SnO}_{2}$ nanocomposite films by flame spray pyrolysis aerosol deposition. (a) Method of micropattering and in situ annealing after deposition of particles. (Adapted from Tricoli [464] with permissions); (b) Supersonic expansion after an FSP reactor. (Adapted from Wegner [476] with permissions).

Fig. 17. Schematic of intrusive diagnostic methods based on sampling. (Adapt from Zhao et al. [483] for (a), Scheckman et al. [490] for (b), Wang et al. [486] for (c), Roth et al.[280] for (g) with permissions).

Fig. 18. Comparison of agglomerate measurements between TEM and DMA-APM. For primary particles (blue filled symbols), the BET-equivalent primary particle size (squares) is consistent with the aerosol (DMA/APM, circles) and thermophoretic (TEM, triangles) sampling result. For agglomerates (black open symbols), the mobility diameter from DMA/APM (circles) agrees well with the project area equivalent diameter from TEM sampling (squares) but differs from the gyration diameter (triangles). The mobility diameter. (Adapted from Gröhn [481] with permission). The inserted figure shows that mobility diameter becomes larger than projected area equivalent diameter as the agglomerates grow from free molecular towards continuum regime. (Adapted from Gröhn [482] with permission).

Fig. 19. Two-dimensional planar measurement of $\mathrm{TiO}_{2}$ nanoparticle volume fraction in a Hencken-stablized Bunsen flame using PS-LIBS. The nozzle outlet is shown as the white line. (Reprinted with permission from Zhang et al. Proc Combust Inst 2015 [296]). 
Fig. 20. Signal intensity of PS-LIBS along the centerline during doping synthesis of $961 \mathrm{ppm}$ TTIP and 314 ppm $\mathrm{VOCl}_{3}$ in a Hencken-stablized Bunsen burner. Ti and $\mathrm{V}$ signals in the doping synthesis process are compared with the signals in the pure synthesis of $\mathrm{Ti}$ and $\mathrm{V}$ (Reprinted with permission from Ren et al. Proc Combust Inst. 2015 [295]).

Fig. 21. Map of different laser-matter interaction of various laser diagnostic methods in flame synthesis. Several typical experimental points are plotted with nondimensional bandgap and laser intensity. PS-LIBS distinguishes from other laser diagnostic methods, as demonstrated by the area that enclosed by the excitation, ablation and breakdown threshold dashed lines.

Fig. 22. Theoretical framework for modeling and simulations of polydisperse nanoparticles.

Fig. 23. The time scales of reaction (red lines), collision (blue lines), sintering (black lines), and fluid convection (green line) as a function of particle diameter at different temperatures in premixed stagnation flame (PSF) aerosol reactor.

Fig. 24. The stationary points on the potential energy surface (PES) of TTIP and main transformation reactions routes of TTIP in $\mathrm{H}_{2} / \mathrm{O}_{2} / \mathrm{Ar}$ flame. (A). The B3LYP calculations predict the effect activation barrier of the TTIP hydrolysis to be ca. $10 \mathrm{kcal} / \mathrm{mol}$. (B) The energy barrier of TTIP thermal decomposition is as large as $85-87 \mathrm{kcal} / \mathrm{mol}$. (C) The scheme of the main transformation reactions of TTIP in H2/O2/Ar flame. (A and B: reprinted, C: adapted, with permission from Shmakov et al. [571]).

Fig. 25. (a) Comparison between van der Waals forces and dipole-dipole forces between two cooriented $3 \mathrm{~nm} \mathrm{TiO}_{2}$ nanoparticles during the approach process. (b) Influence of dipole-dipole interaction on enhancement factors of coagulation at different temperatures. Subplots A and B show the forces between $\mathrm{TiO}_{2}$ nanoparticles at 273 and 1273K, respectively. (Adapted from Yan et al. [590] and 
Zhang et al.[591] with permissions).

Fig. 26. Map of grain structure morphologies of $\mathrm{TiO}_{2}$ for different diameters and temperatures; A stands for amorphous morphology; Core-shell stands for crystalline-core-amorphous-shell morphology; $\mathrm{L}$ stands for liquid phase; and $\mathrm{S}$ stands for solid phase. Inserted figure (a) shows a crystalline-core-amorphous-shell structure of a $3 \mathrm{~nm}$ nanoparticle and (b) shows an amorphous structure of a $2 \mathrm{~nm}$ nanoparticle. (Adapted from Zhang et al. [593] with permissions).

Fig. 27. The normalized surface area and temperature during the sintering process of two $3 \mathrm{~nm} \mathrm{TiO}_{2}$ nanoparticles. Snapshots of nanoparticles at $\mathrm{t}=0,0.5,1,8$ and $15 \mathrm{~ns}$ are shown in the inserted pictures where blue and white spheres are $\mathrm{O}$ and $\mathrm{Ti}$ atoms, respectively, in the certain crystalline region; red and pink spheres are $\mathrm{O}$ and $\mathrm{Ti}$ atoms, respectively, in the outer amorphous region. 


\section{Figures}

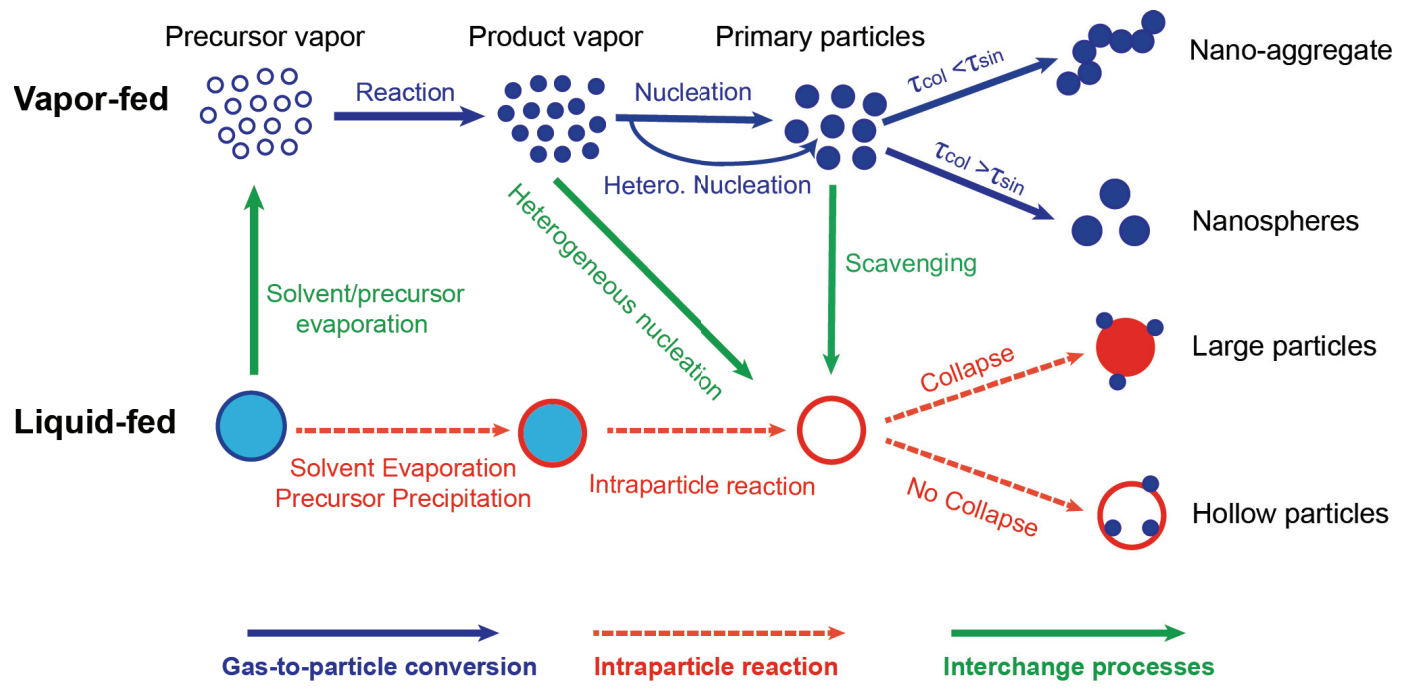

Fig. 1. Schematic of particle formation processes during vapor-fed or liquid-fed flame synthesis through mechanisms of gas-to-particle conversion, intra-particle reaction, etc. 
$\begin{array}{ll}\text { (a) Coflow diffusion flame } & \text { (b) Mckkena flat flame }\end{array}$
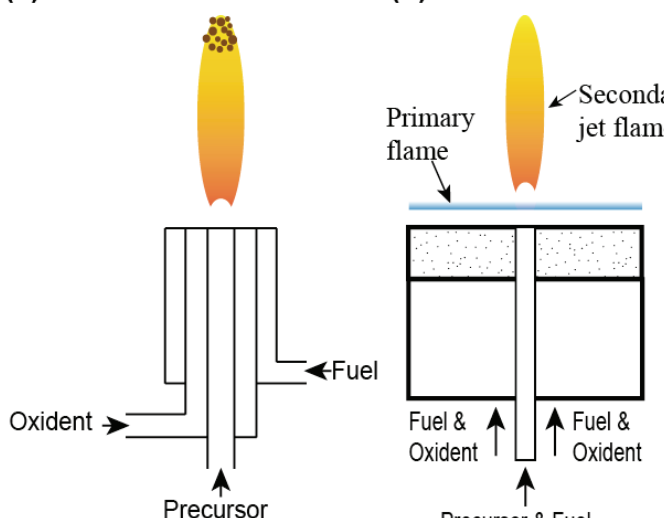

Precursor \& Fuel (c) Hecken flat flame

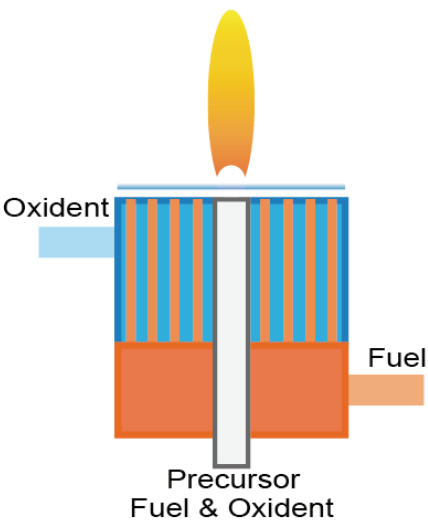

(d) Counterflow flame

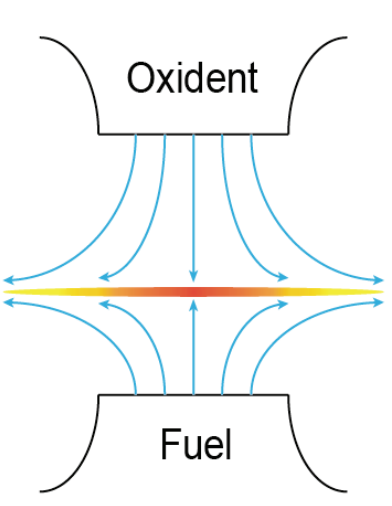

(f.1) Stagnation premixed: burner-stabilization
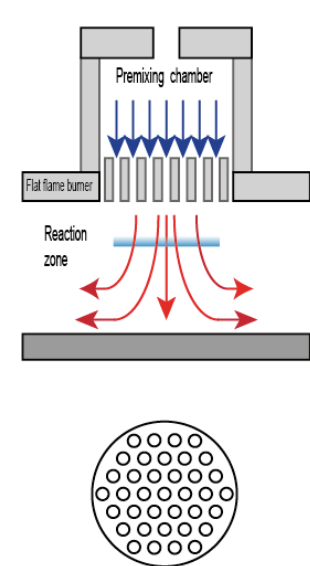

inner-tube-stabilization

Combustion Ga
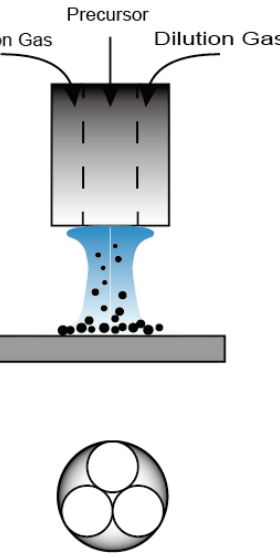

divergence-stabilization
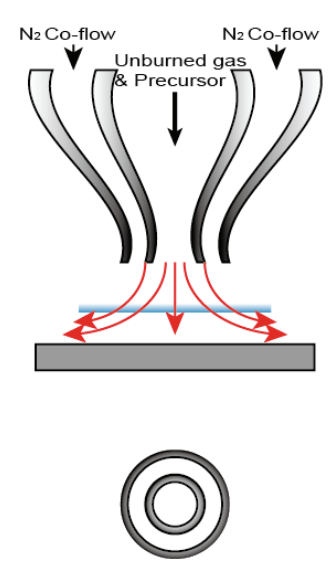

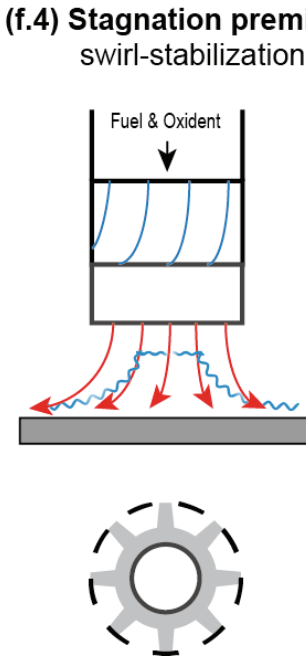

(e) Flame spray pyrolysis

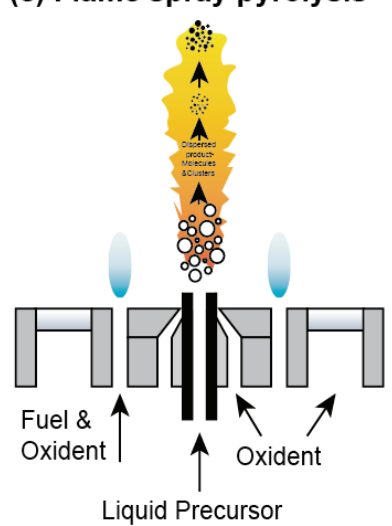

(g) Stagnation flame: spray pyrolysis

Liquid Precursor
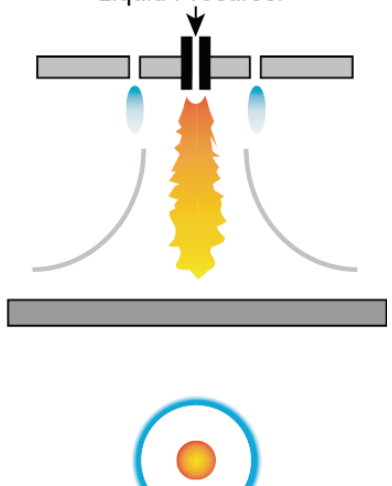

Fig. 2. Schematics of various typical burner setups used in flame synthesis. 


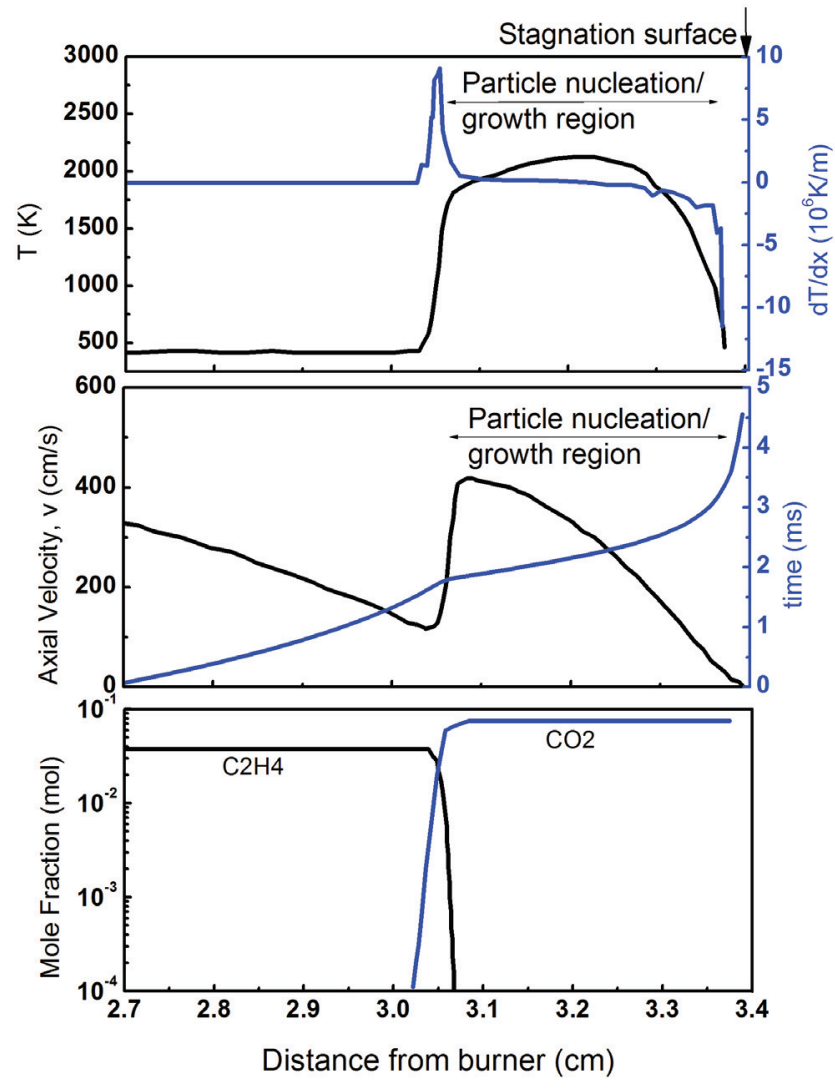

Fig. 3. The temperature, fluid flow and species mole fractions fields of classical 1-D stagnation premixed flames along the centerline of a typical premixed stagnation flame (Mixture: $3.96 \%-\mathrm{C}_{2} \mathrm{H}_{4} / 26.53 \%-\mathrm{O}_{2} / 69.51 \%$-Ar; Equivalence ratio: $\Phi=0.45$; Configuration: I.D. $1 \mathrm{~cm}$ nozzle with nozzle-substrate distance $3.4 \mathrm{~cm}$ ), as adapted from literature [311]. The steepest temperature gradient at the cold stagnation plate reaches as large as $5 \times 10^{4} \mathrm{~K} / \mathrm{cm}$. 

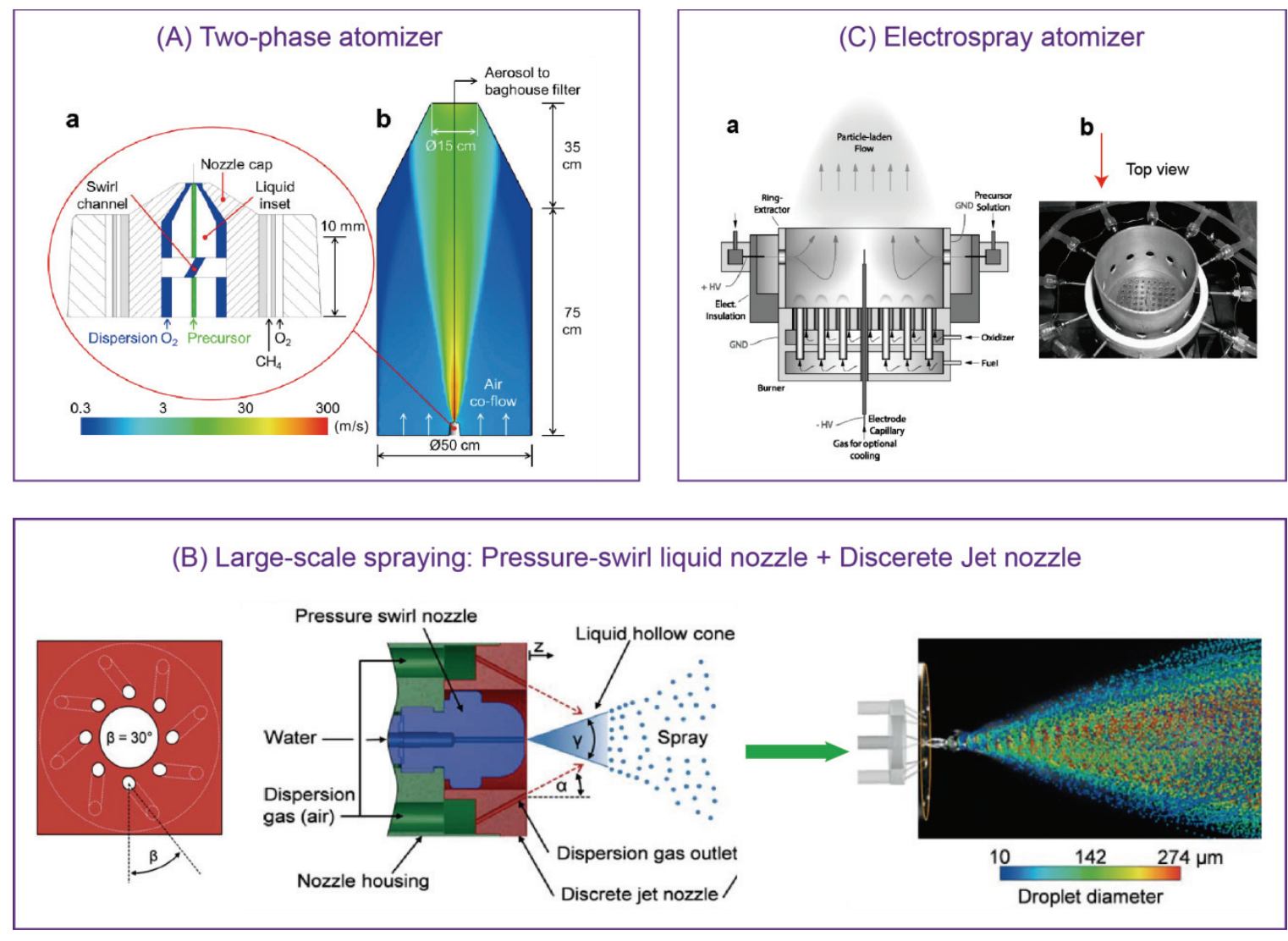

Fig. 4. Techniques of various spraying atomizers used in flame spray pyrolysis. (A) Lab-scale two-phase atomizer, with liquid precursor delivered through the center capillary and dispersed with oxygen, along with its location in a cylindrical reactor chamber [329]; (B) Large-scale novel atomization combining a pressure swirl nozzle (liquid hollow cone nozzle) and a dispersion gas nozzle, where $\alpha$ is the gas inclination angle, $\beta$ is the gas swirl angle and $\gamma$ is the for liquid hollow cone angle [331]; (C) Lab-scale electrospray atomizer [195]. (Reprinted with permission from literature [195][329][331]). 
(A) $\mathrm{Pd} / \mathrm{TiO}_{2}$

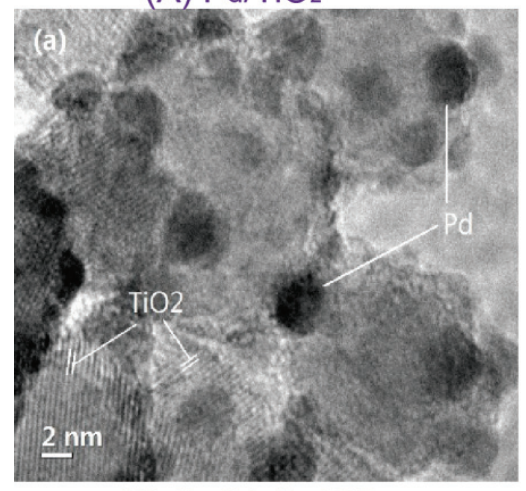

(C) $\mathrm{Pd} / \mathrm{Al}_{2} \mathrm{O}_{3}-\mathrm{SiO}_{2}$

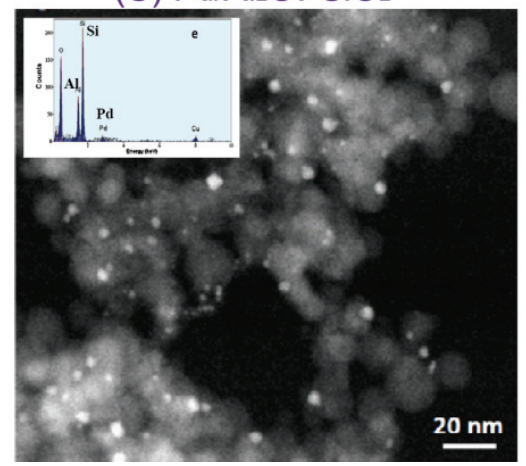

(B) $\mathrm{Pt}-\mathrm{Pd} / \mathrm{Al}_{2} \mathrm{O}_{3}$

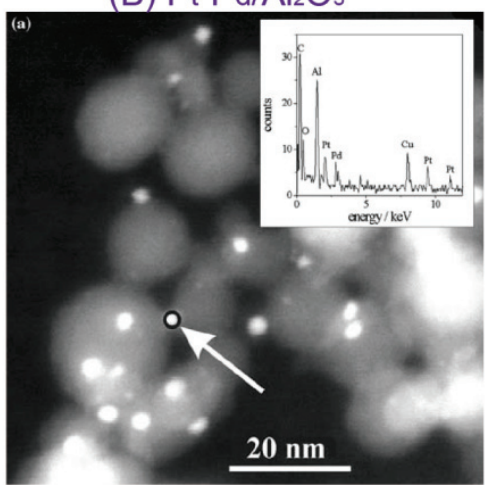

(D) $\mathrm{La}(\mathrm{Mn}, \mathrm{Pd}) \mathrm{O}_{3 \pm \overline{0}}$

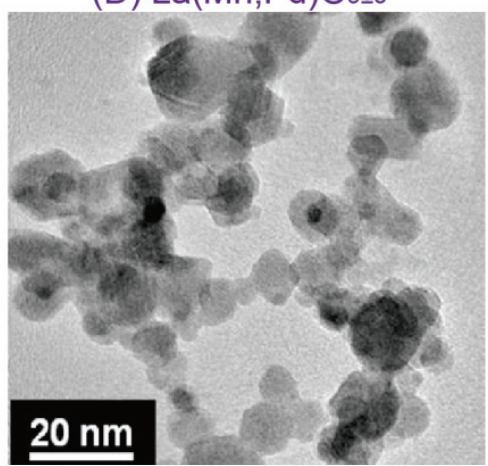

(E) $\mathrm{Pt}-\mathrm{Ba} / \mathrm{Ce}_{x} \mathrm{Zr}_{1-\mathrm{x}} \mathrm{O}_{2}$

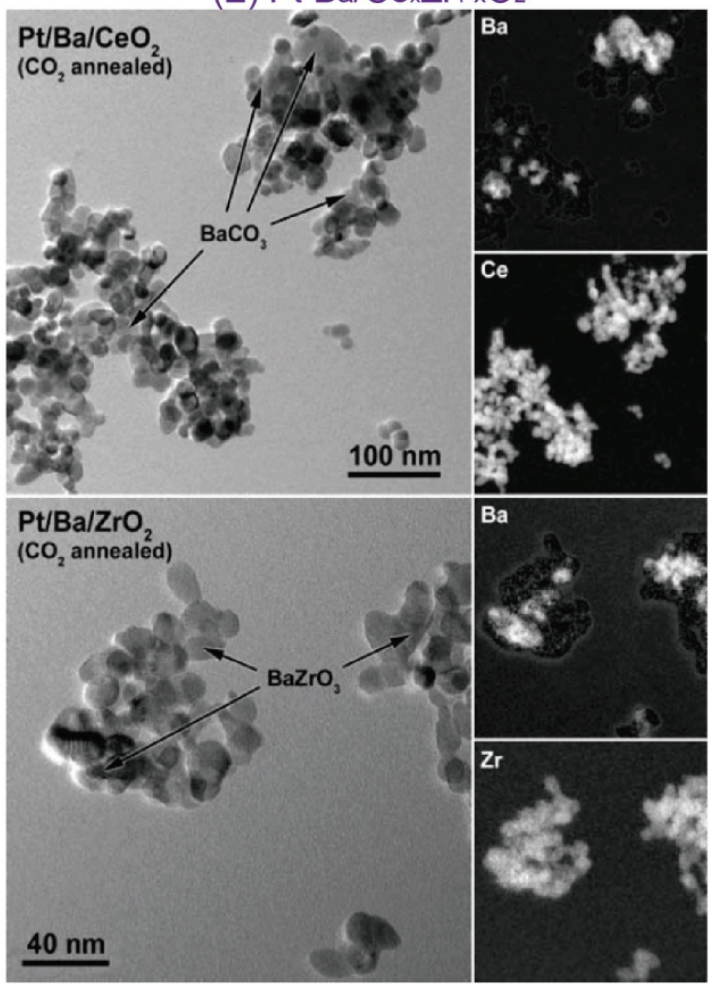

Fig. 5. Flame-made nanocomposite materials of metal-support mixture with heterogeneously dispersed structures. A: $\mathrm{TiO}_{2}$ supported Pd nanoparticles made by Niu et al. JPCC, 2014 [112]; $\mathrm{B}: \mathrm{Al}_{2} \mathrm{O}_{3}$ supported Pt-Pd alloy particles made by Strobel et al., Catal. Lett. 2005 [69]; C: Pd nanoparticles on mixed $\mathrm{SiO}_{2}-\mathrm{Al}_{2} \mathrm{O}_{3}(\mathrm{Pd} / \mathrm{SA})$ with tunable surface acidity made by Huang et al., J. Catal. 2011 [73]; D: Pd-containing perovskite-type oxide catalyst made by Lu et al., Appl. Catal. B. 2014 [358]; E: Pt/Ba on $\mathrm{CeO}_{2}-\mathrm{ZrO}_{2}$ mixed support by Strobel et al. J. Catal. 2006 [203]. (Reprinted with permission from literature [112] [69] [73] [358] [203]). 
(A) $\left(\mathrm{Ce}_{0.7} \mathrm{Zr}_{0.3} \mathrm{O}_{2}\right)_{0.5}\left(\mathrm{~A}_{12} \mathrm{O}_{3}\right)_{0.5}$

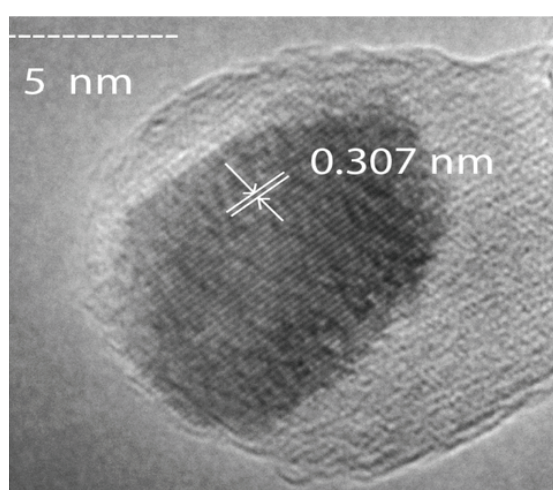

(B) $\mathrm{TiO}_{2} / \mathrm{CeO}_{2}$

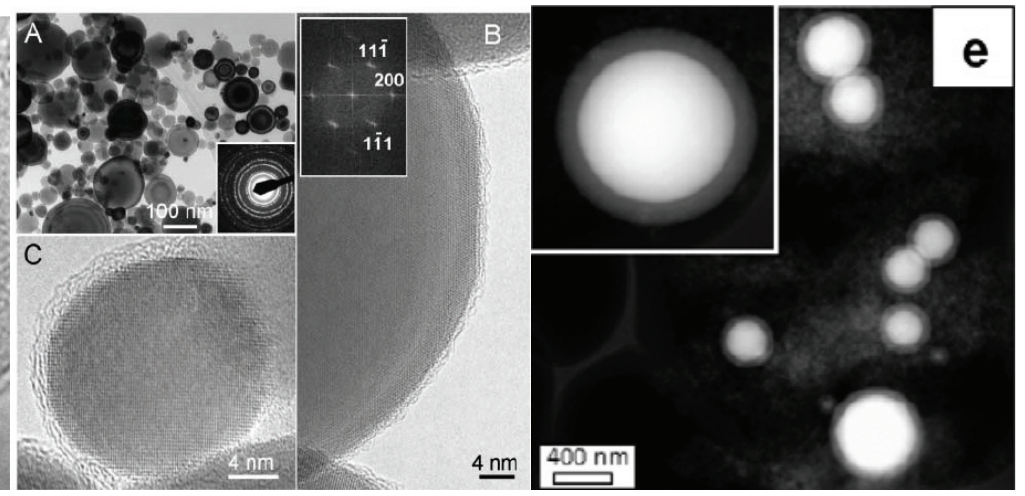

Fig. 6. Flame-made nanocomposite materials with core-shell structures. (A): Shell- $\left(\mathrm{Ce}_{0.7} \mathrm{Zr}_{0.3} \mathrm{O}_{2}\right) 0.5$ / Core- $\left(\mathrm{Al}_{2} \mathrm{O}_{3}\right) 0.5$ by Weidenhof et al., JACS, 2009, 131, p9207 [35]; (B): Shell- $\mathrm{TiO}_{2}$ (12 atomic \%)/Core- $\mathrm{CeO}_{2}$ by Feng et al., Science, 2006 [12]; C: Shell- $\mathrm{SiO}_{2} /$ Core- $\mathrm{Y}_{2} \mathrm{Si}_{2} \mathrm{O}_{7}:$ Eu, Hasegawa et al. Proc. Combust. Inst., 2011 [189]. (Reprinted with permission from literature [12][35][189]). 
(A) TEM, HRTEM and SAED patterns

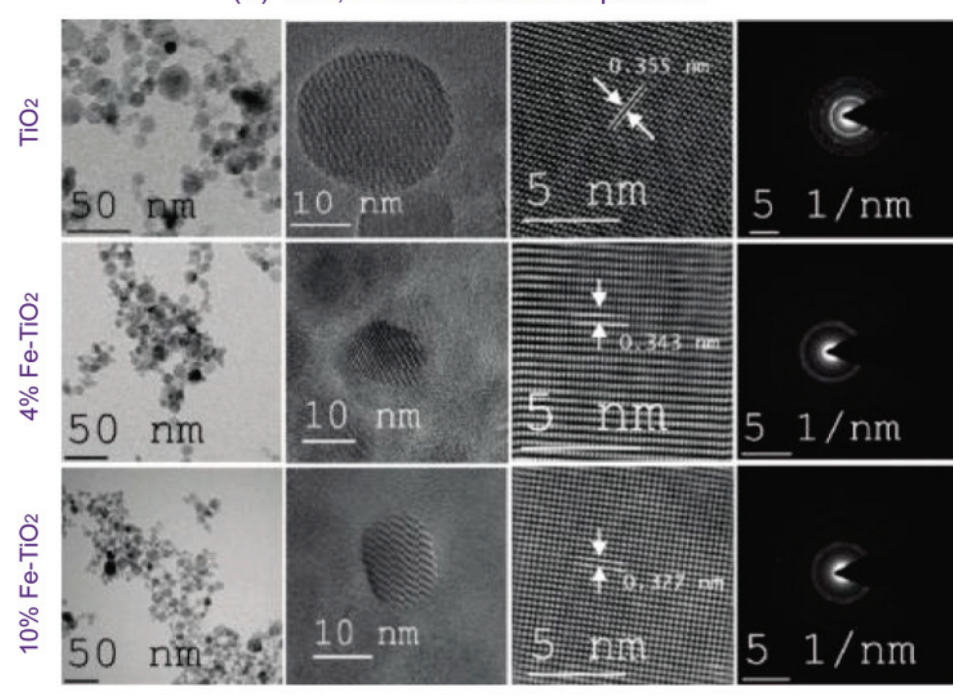

(B) EFTEM for $10 \% \mathrm{Fe}-\mathrm{TiO}_{2}$

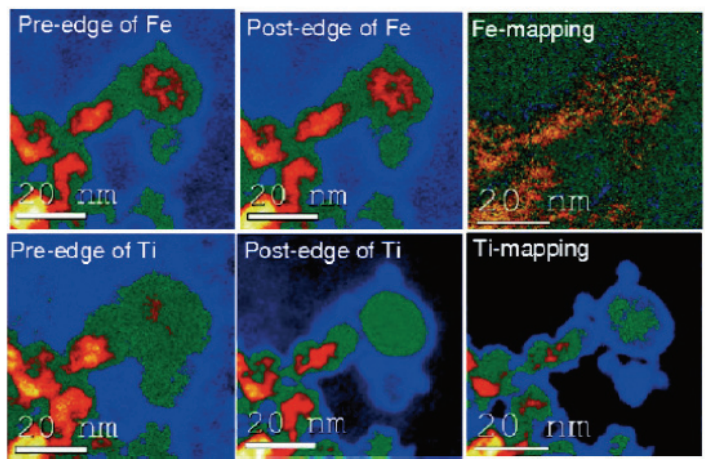

Fig. 7. Flame-made nanocomposite materials with homogenously-mixed single-phase structures. (A) low-resolution and high resolution TEM images of single particles, with magnified portion of the particles and respective SAED patterns of pure $\mathrm{TiO}_{2}$ (upper panel), 4 atom $\% \mathrm{Fe}$-doped $\mathrm{TiO}_{2}$ (middle panel), and 10 atom \% Fe-doped $\mathrm{TiO}_{2}$ (lower panel); (B) Energy-filtered TEM (EFTEM) of 10 atom \% Fe-doped $\mathrm{TiO}_{2}$ nanoparticles. (Reprinted with permission from literature by George et al. JACS, 2011 [105]). 


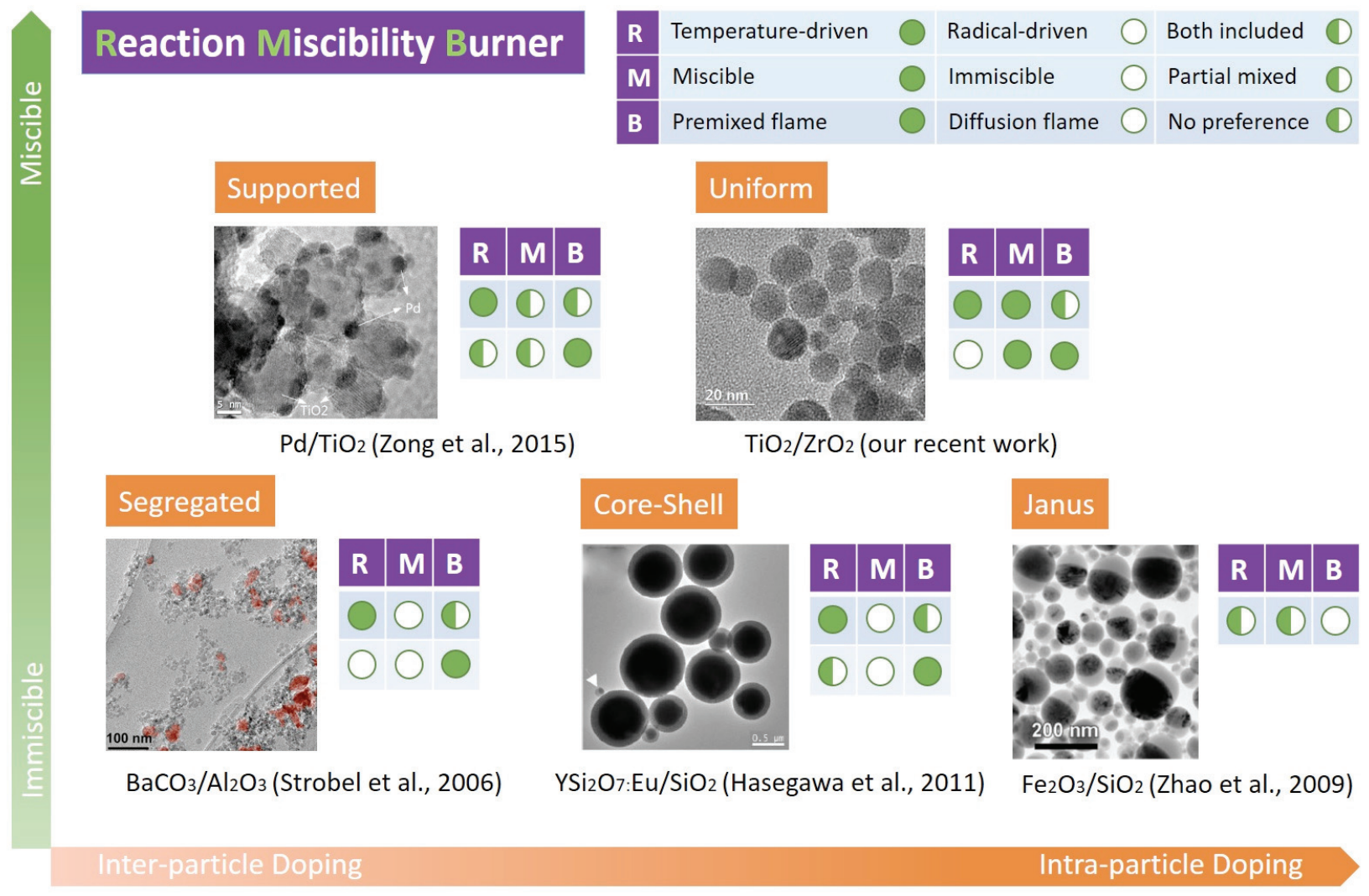

Fig. 8. Doping diagram of flame synthesis for achieving various hierarchical structures. 

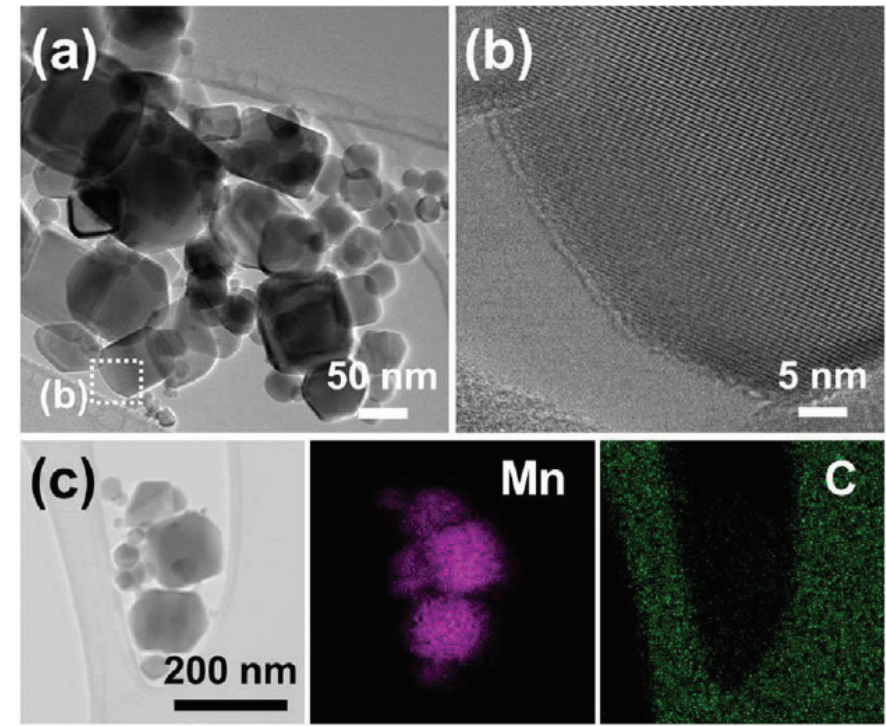

Fig. 9. Morphologies and dot-mapping images of dispersed Carbon-Mn3O4 composite structure prepared by flame spray pyrolysis: (a) TEM image, (b) high-resolution TEM image, and (c) dot-mapping images. (Reprinted with permission from Ko et al. Scientific Reports 2014 [375]). 


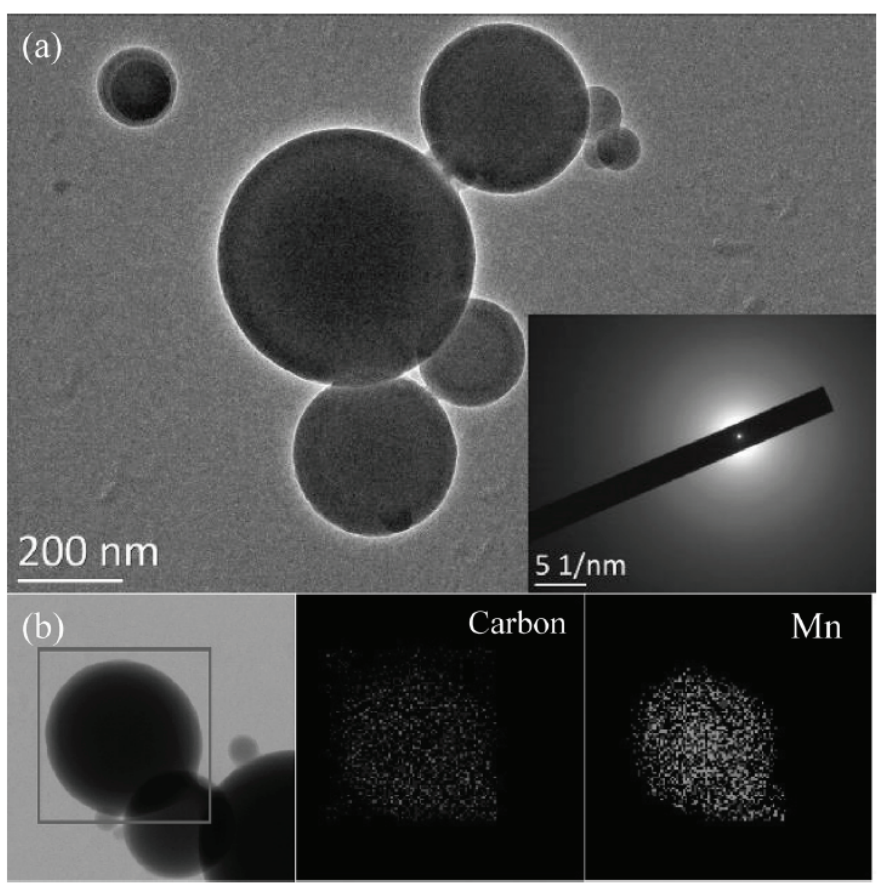

Fig. 10. Morphologies and dot-mapping images of amorphous carbon-MnOx nanocomposite particles:

(a) TEM and SAED images; (b) EDS mapping image of distribution of elemental carbon and Mn. (Reprinted with permission from Guo et al. Adv. Funct. Mater. 2012 [376]). 

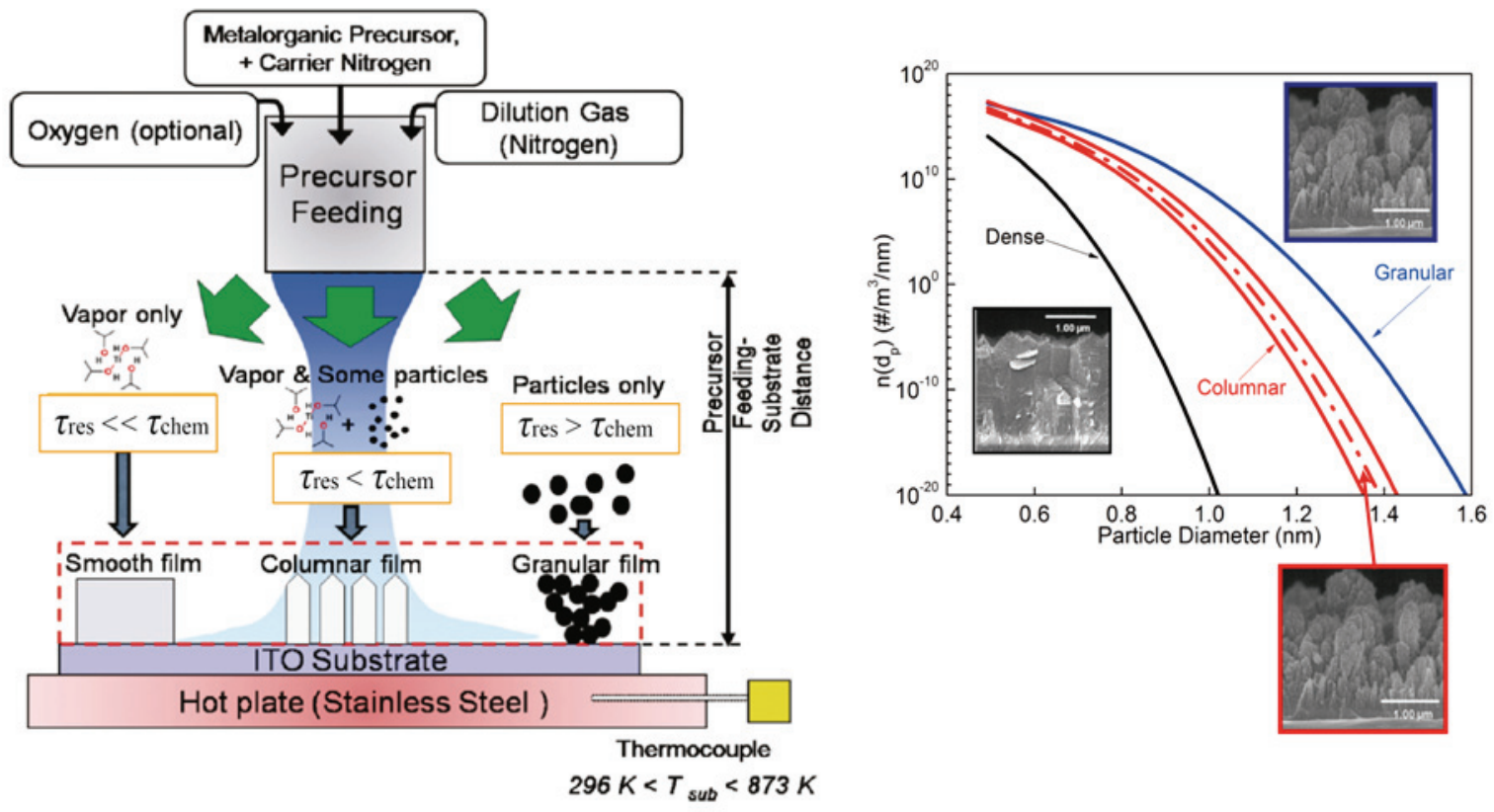

Fig. 11. Illustration of three deposition and film growth mechanisms (left part) and its controlling diagram as functions of particle diameter and size distribution function of particle number concentration. When the residence time is much smaller than the chemical reaction time, vapor deposits on the plate and forms the dense smooth film which corresponed to "chemical vapor deposition"; When the residence time is larger than the chemical reaction time, as-synthesized particles deposit on the plate and form the dense granular film which correspond to "aerosol deposition"; When the residence time is smaller or comparative to the chemical reaction time, mixture of vapor and particles deposits on the plate and forms the columnar film which corrpond to the mix deposition regime. (Reprinted from An et al. 2009, [428]). 

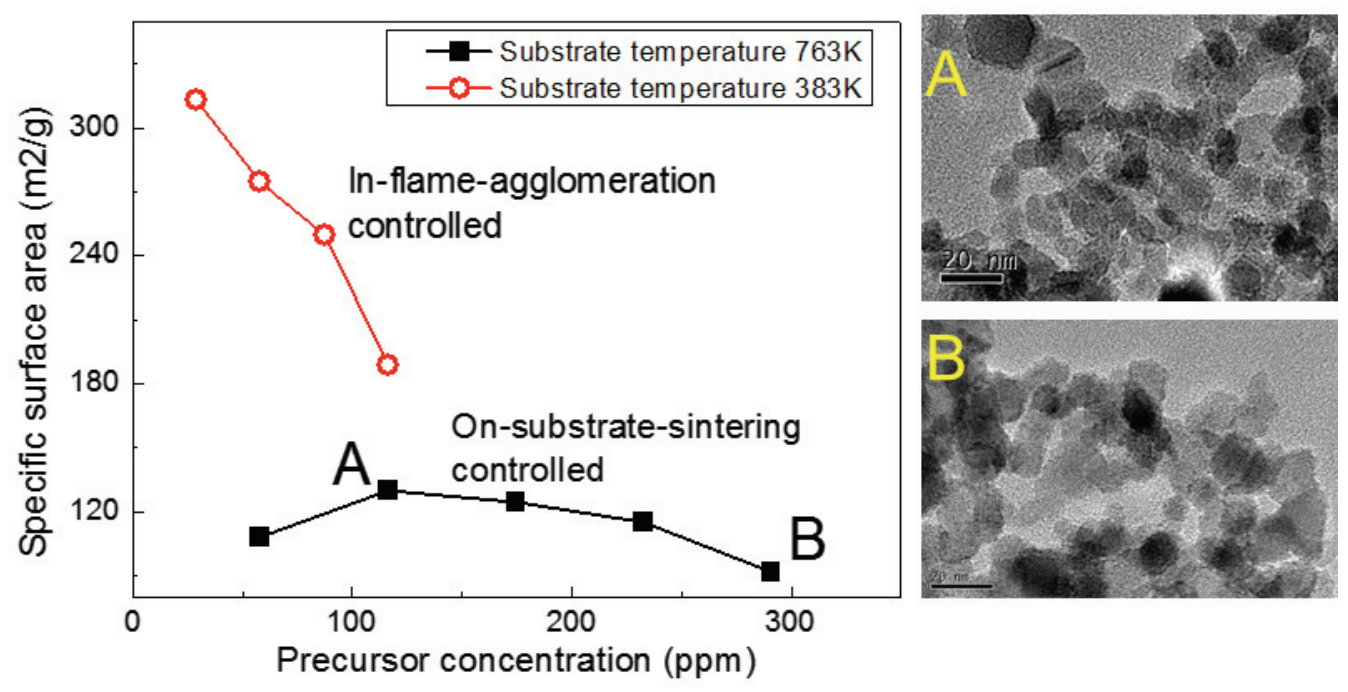

Fig. 12. Specific surface area (SSA) of $\mathrm{TiO}_{2}$ nanofilms (granular-regime) under different substrate temperatures. Open circles represent the data for a substrate temperature of $383 \mathrm{~K}$, while the filled squares correspond to a substrate temperature of $763 \mathrm{~K}$. TEM images on the right-hand side show the morphologies of the nanofilms at (A) $116.4 \mathrm{ppm}$ and (B) $291.0 \mathrm{ppm}$, for a substrate temperature of $763 \mathrm{~K}$. (Reprinted with permission from Zhang et al. 2012 [10]). 
A. Boundary layer for nanoparticle deposition

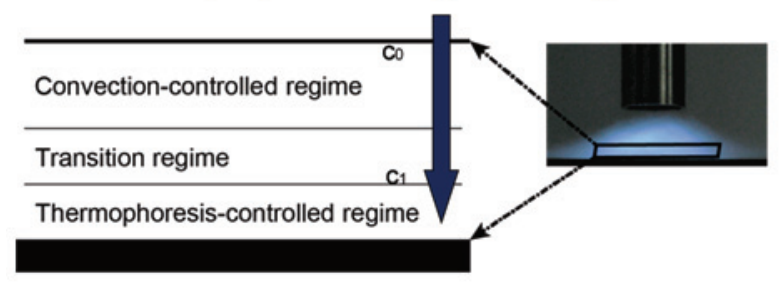

B. Gas velocity vs. Thermphoretic velocity

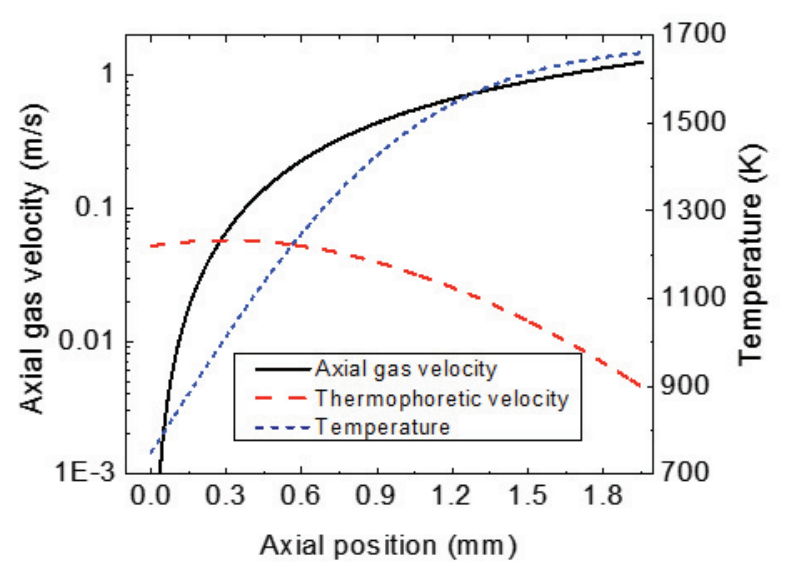

Fig. 13. Characterization of the laminar boundary layer above a stagnation plane for particle deposition by comparing the profiles between gas velocity and thermophoretic velocity. (The Renolds number for the stagnation jet is around 1500 , the stagnation strain rate is around $60 \mathrm{~s}^{-1}$, and the tempartue of gas outside the boundary layer about $1700 \mathrm{~K}$ ) (Reprinted with permission from Zhang et al. 2012, [323]). 


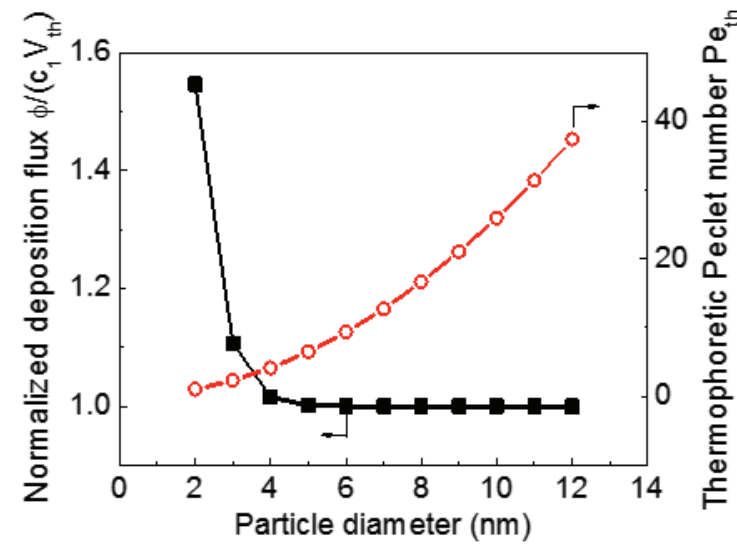

Fig. 14. The variation of the normalized deposition flux $\phi / c_{1} V_{\text {th }}$ obtained from Eqn. (5.7) and corresponding the thermophoretic Peclet number, $\mathrm{Pe}_{\mathrm{th}}$, as a function of nanoparticle diameter (Reprinted with permission from Zhang et al. 2012 [323]). 
(A) Stagnation flame aerosol deposition

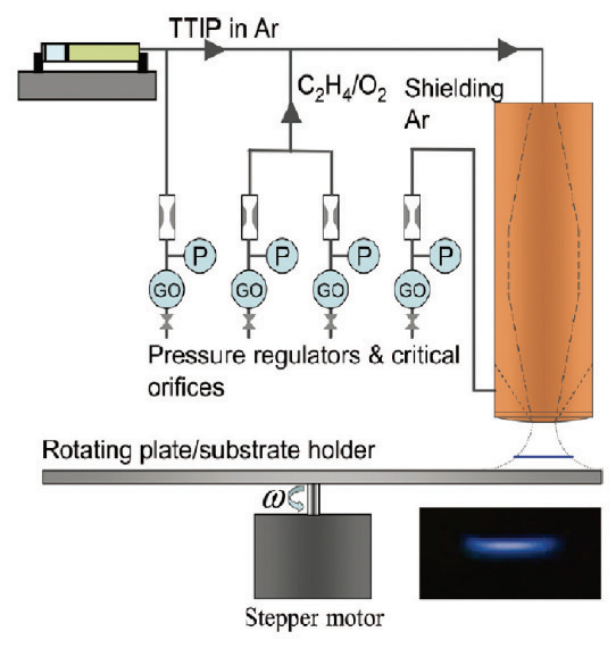

(B) Gas sensing of $\mathrm{CO}$

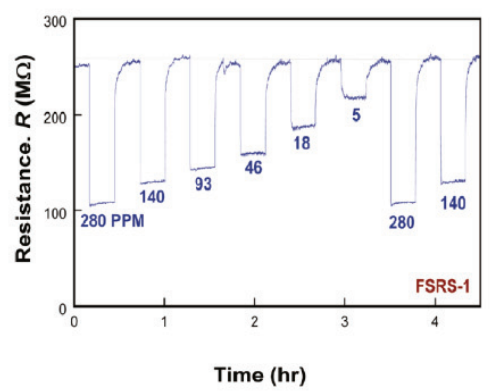

(D) Densitification and sintering of synthesized films

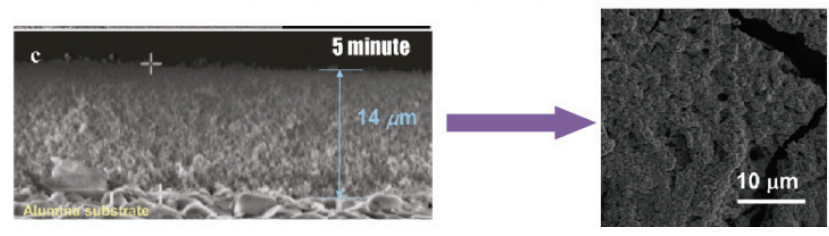

Fig. 15. Fabrication of $\mathrm{TiO}_{2}$ films by vapor-fed stagnation flame aerosol deposition for both $\mathrm{CO}$ gas sensing and dye-sensitized solar cell applications. The right (A) shows the stagnation flame aerosol deposition by a premixed stagnation flame seeded with TTIP (adapted from Wang and co-workers [318] with permissions); The middle top (B) shows the reponses to $\mathrm{CO}$ exposure at $773 \mathrm{~K}$ for sensor films (adapted from Wang and co-workers [461] with permissions); The right top (C) shows photocurrent density-voltage characteristics of dye sensitized solar cells (adapted from Wang and co-workers [462] with permissions); The bottom right shows the SEM images of mesoporous $\mathrm{TiO}_{2}$ films before and after densification and sintering (adapted from Wang and co-workers [461] for the right part and [462] for the left partwith permissions). 


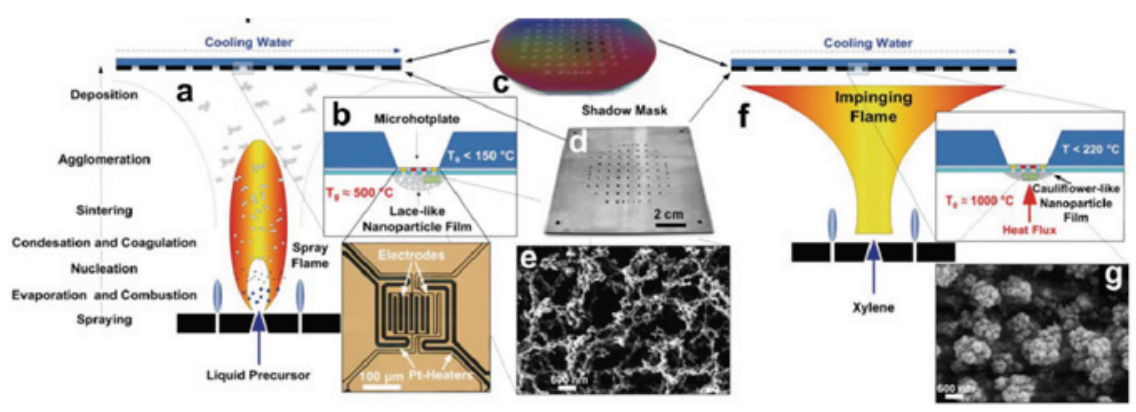

(a) Micropattering and in situ annealing

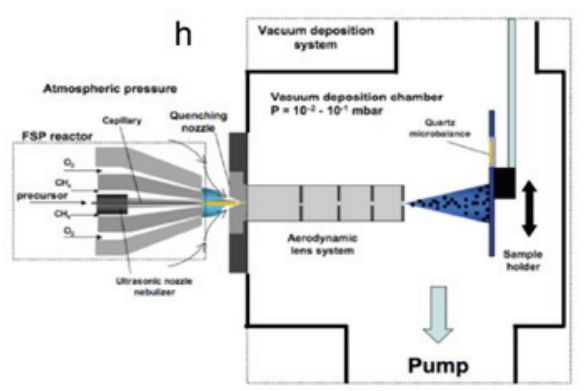

(b) Supersonic expansion

Fig. 16. Fabrication of $\mathrm{SnO}_{2}$ nanocomposite films by flame spray pyrolysis aerosol deposition. (a) Method of micropattering and in situ annealing after deposition of particles. (Adapted from Tricoli [464] with permissions); (b) Supersonic expansion after an FSP reactor. (Adapted from Wegner [476] with permissions). 
(a) Dilution sampling and SMPS

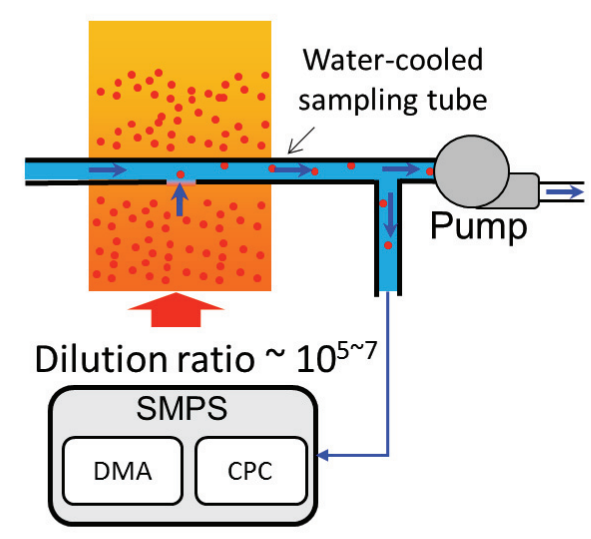

(b) Dilution sampling and DMA-APM system

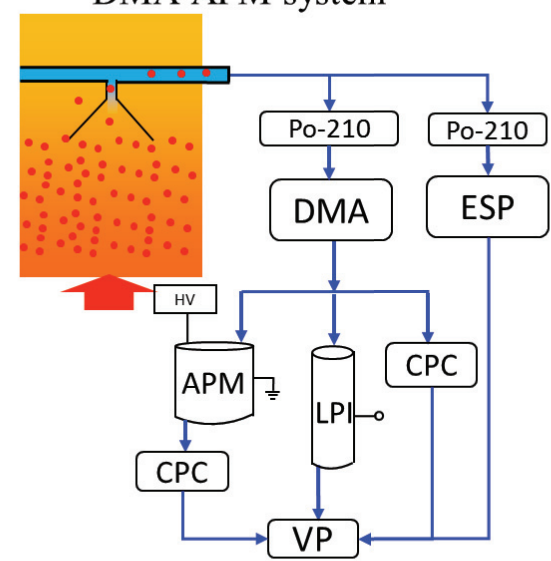

(e) Samples from Direct deposition

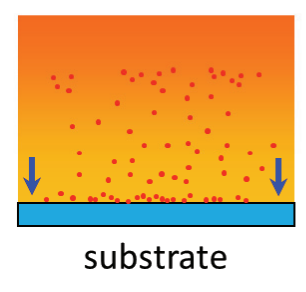

(f) Samples from Filters

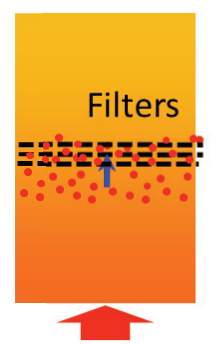

(c) One-stage dilution sub2nm sampling system

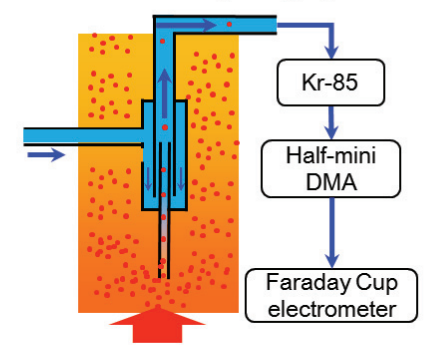

(d) TEM in-situ sampling system

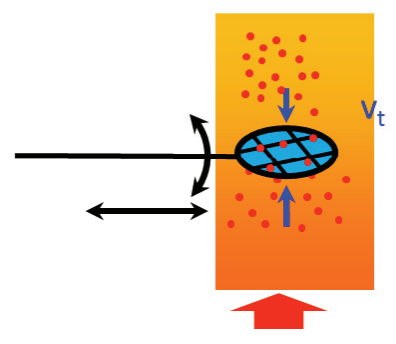

Fig. 17. Schematic of intrusive diagnostic methods based on sampling. (Adapt from Zhao et al. [483] for (a), Scheckman et al. [490] for (b), Wang et al. [486] for (c), Roth et al.[280] for (g) with permissions). 


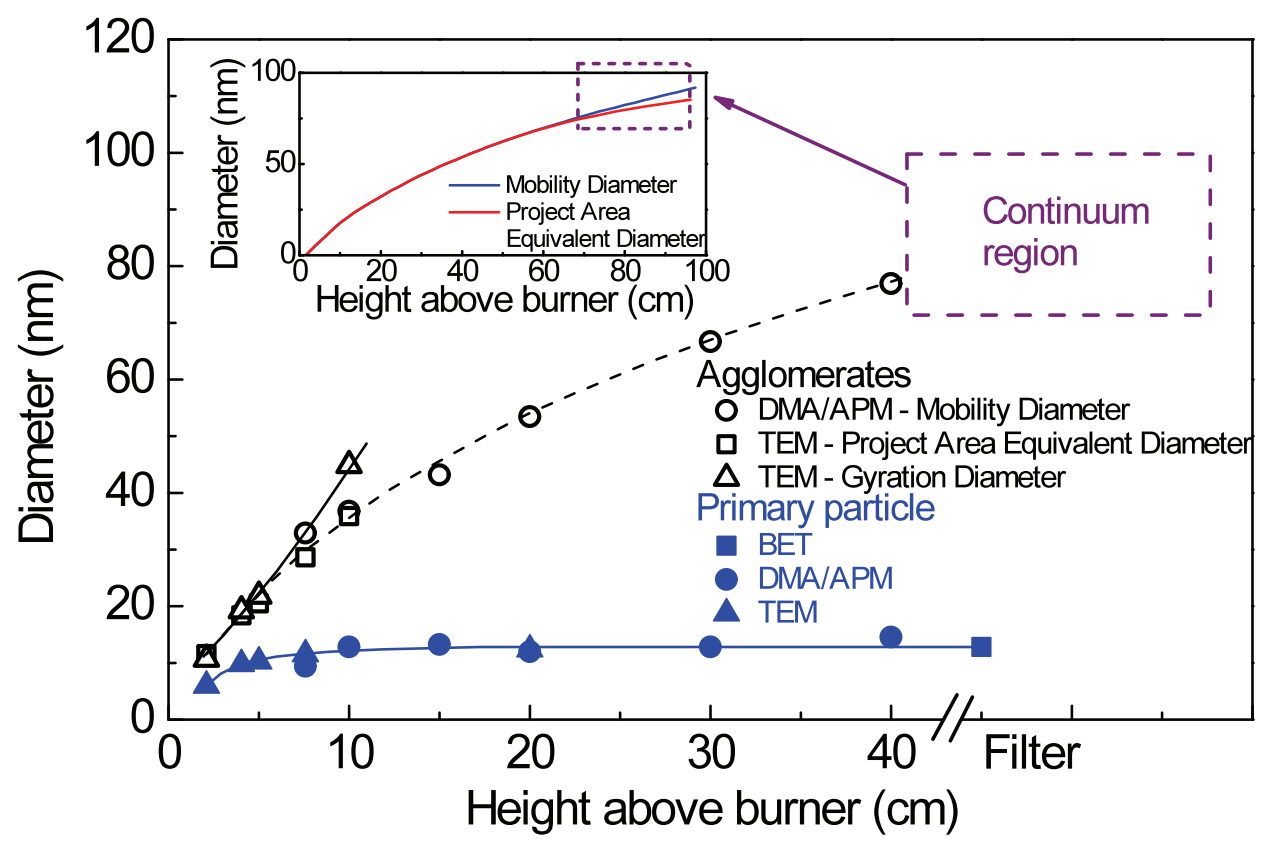

Fig. 18. Comparison of agglomerate measurements between TEM and DMA-APM. For primary particles (blue filled symbols), the BET-equivalent primary particle size (squares) is consistent with the aerosol (DMA/APM, circles) and thermophoretic (TEM, triangles) sampling result. For agglomerates (black open symbols), the mobility diameter from DMA/APM (circles) agrees well with the project area equivalent diameter from TEM sampling (squares) but differs from the gyration diameter (triangles). The mobility diameter. (Adapted from Gröhn [481] with permission). The inserted figure shows that mobility diameter becomes larger than projected area equivalent diameter as the agglomerates grow from free molecular towards continuum regime. (Adapted from Gröhn [482] with permission). 


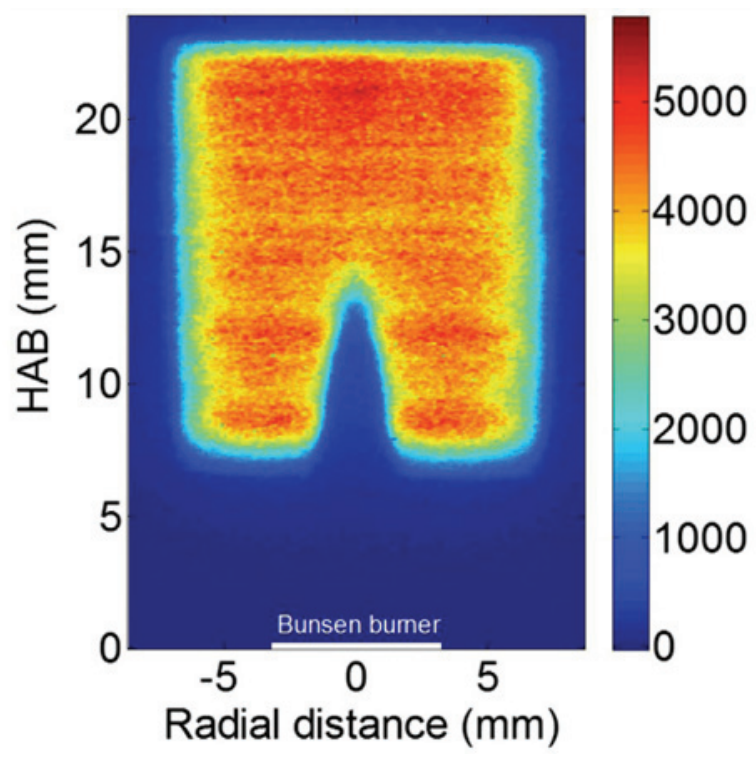

Fig. 19. Two-dimensional planar measurement of $\mathrm{TiO}_{2}$ nanoparticle volume fraction in a Hencken-stablized Bunsen flame using PS-LIBS. The nozzle outlet is shown as the white line. (Reprinted with permission from Zhang et al. Proc Combust Inst 2015 [296]). 


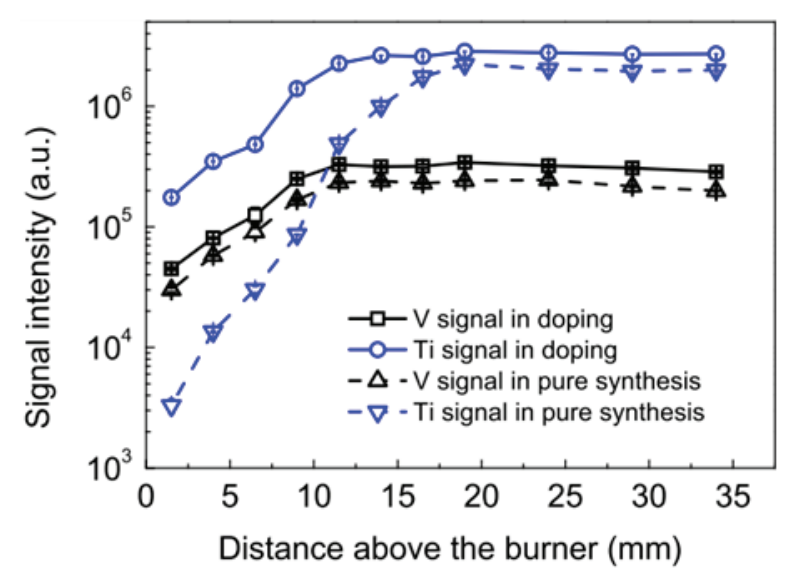

Fig. 20. Signal intensity of PS-LIBS along the centerline during doping synthesis of $961 \mathrm{ppm}$ TTIP and $314 \mathrm{ppm} \mathrm{VOCl}_{3}$ in a Hencken-stablized Bunsen burner. Ti and $\mathrm{V}$ signals in the doping synthesis process are compared with the signals in the pure synthesis of Ti and V. (Reprinted with permission from Ren et al. Proc Combust Inst. 2015 [295]). 


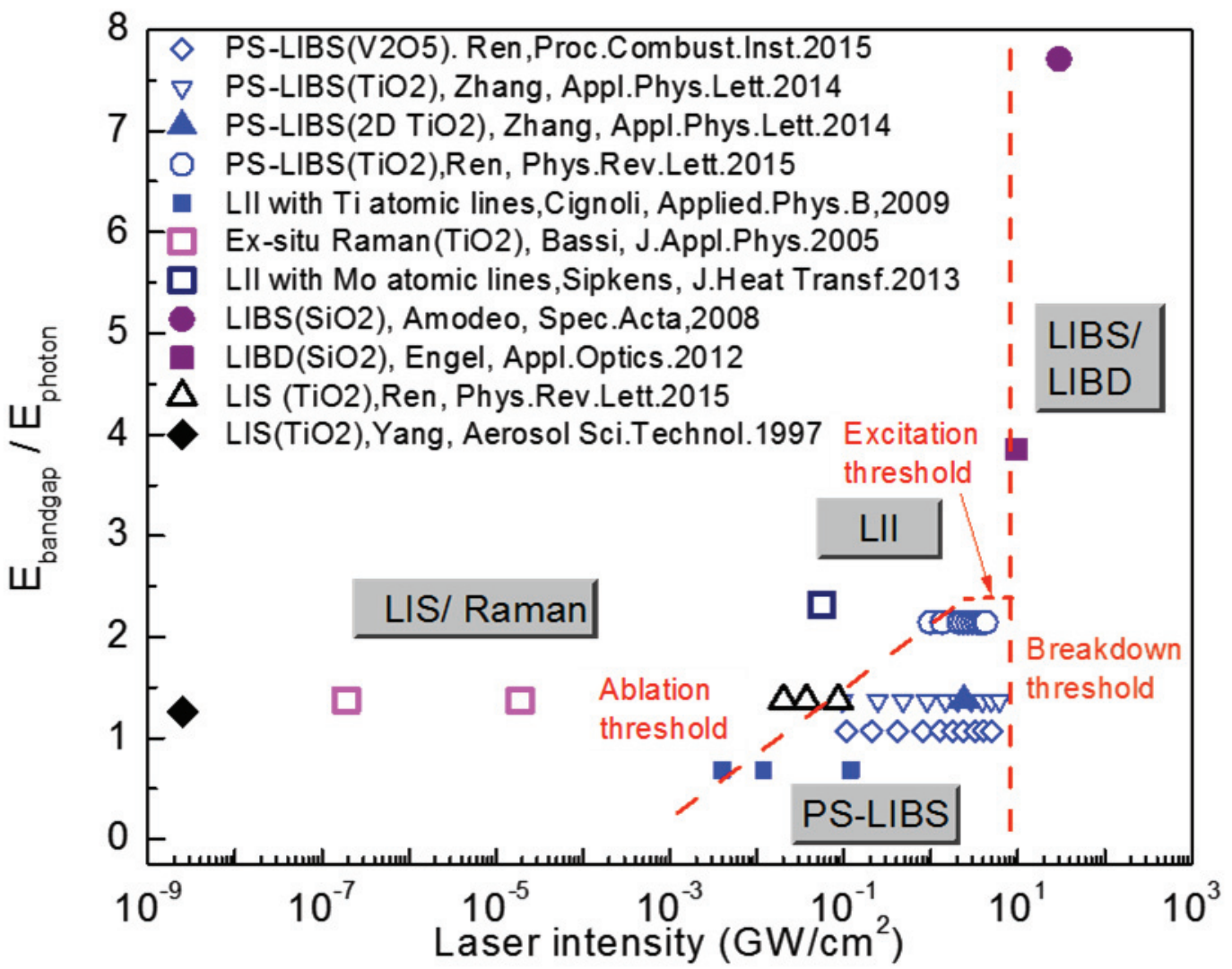

Fig. 21. Map of different laser-matter interaction of various laser diagnostic methods in flame synthesis. Several typical experimental points are plotted with nondimensional bandgap and laser intensity. PS-LIBS distinguishes from other laser diagnostic methods, as demonstrated by the area that enclosed by the excitation, ablation and breakdown threshold dashed lines. 


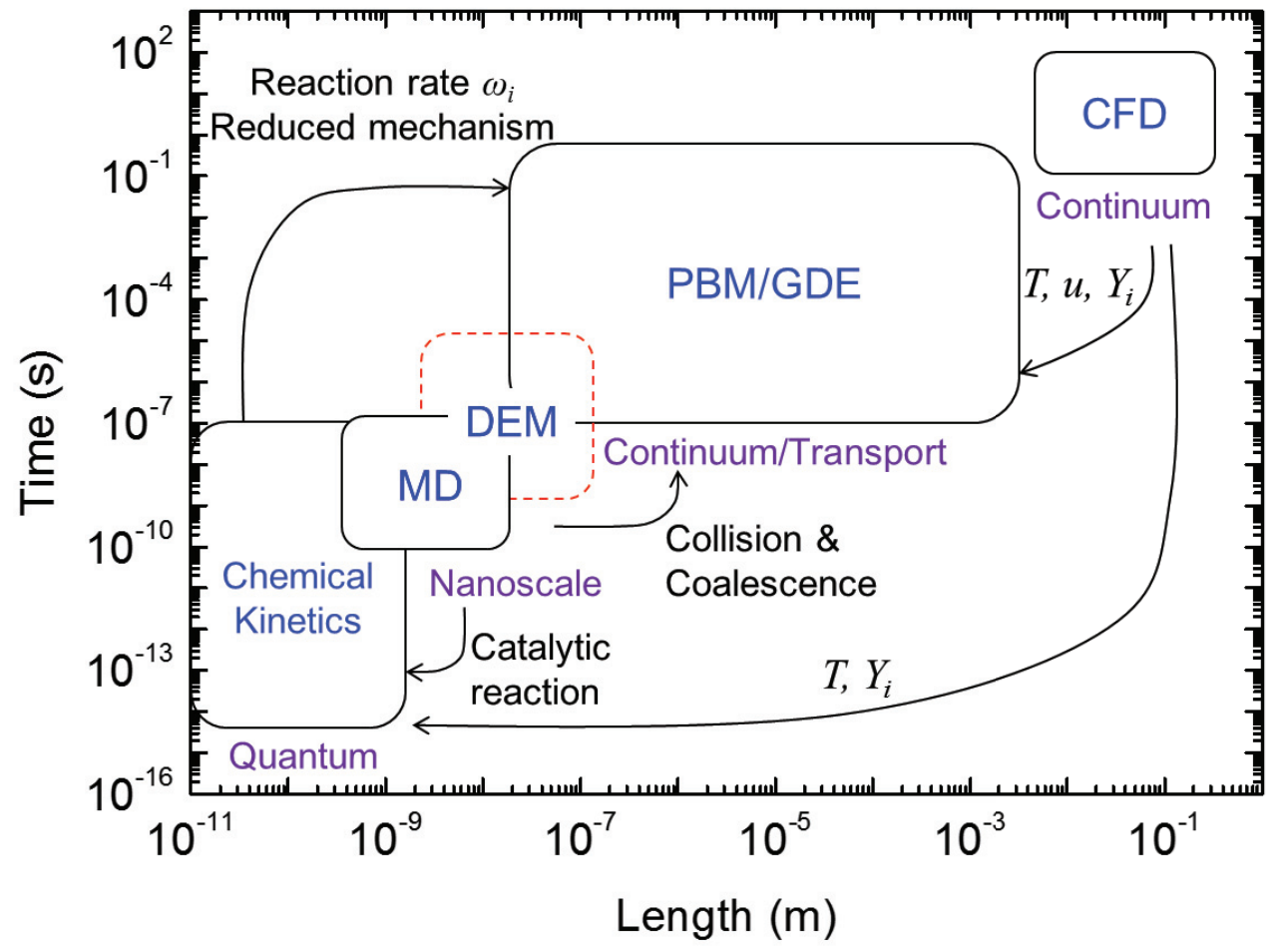

Fig. 22. Theoretical framework for modeling and simulations of polydisperse nanoparticles. 


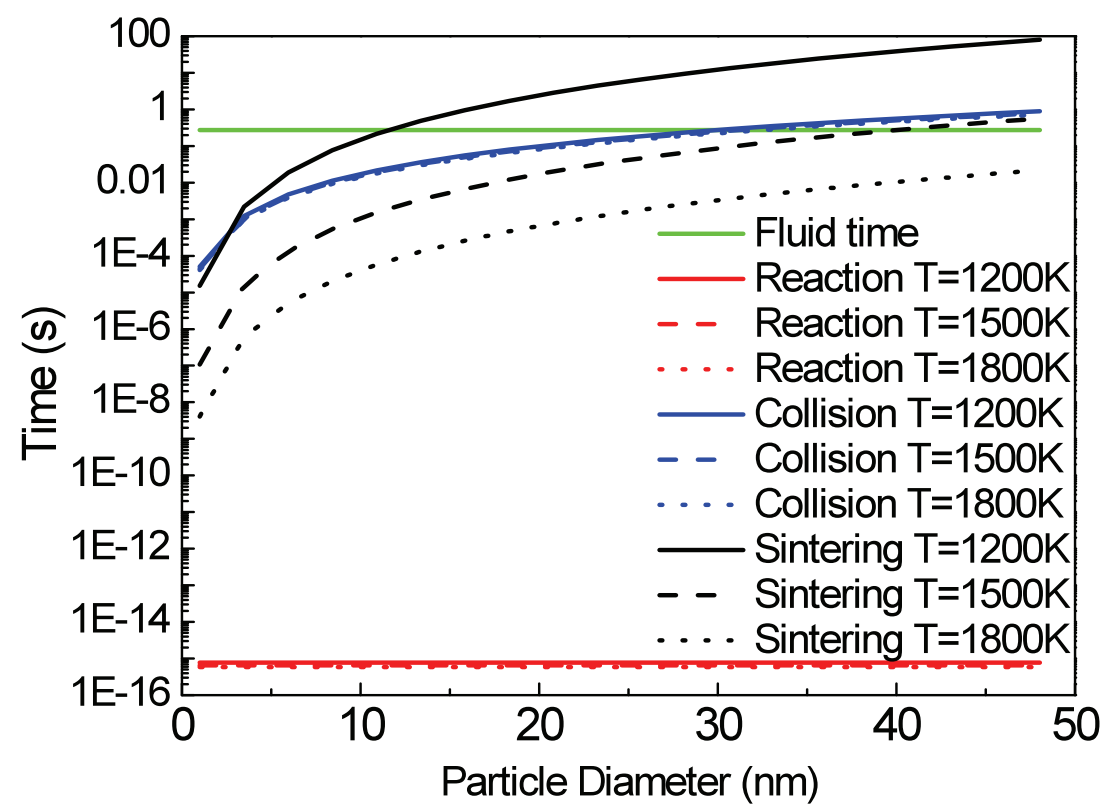

Fig. 23. The time scales of reaction (red lines), collision (blue lines), sintering (black lines), and fluid convection (green line) as a function of particle diameter at different temperatures in premixed stagnation flame (PSF) aerosol reactor. 
A. The stationary points on the PES corresponding to the hydrolysis of TTIP

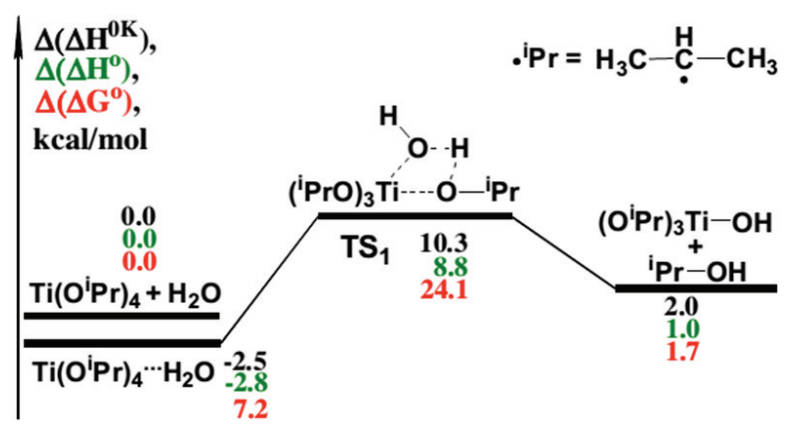

B. The stationary points on the PES corresponding to monomolecular reactions

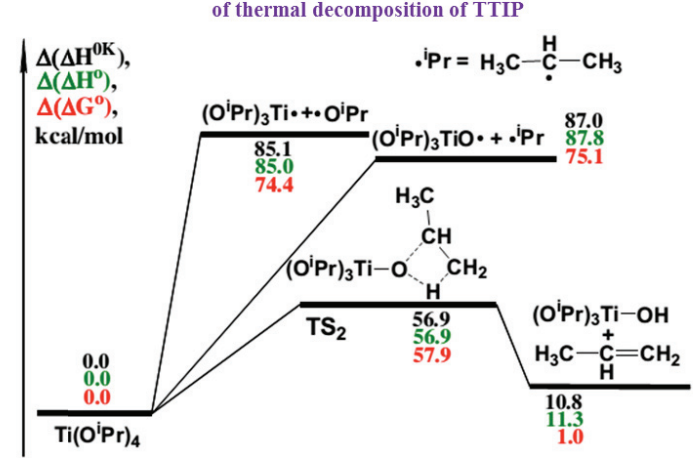

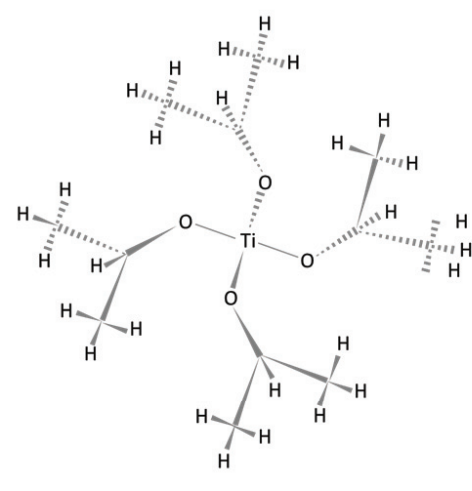

C. The scheme of the main transformation reeactions of TTIP in $\mathrm{H}_{2} / \mathrm{O}_{2} / \mathrm{Ar}$ flame

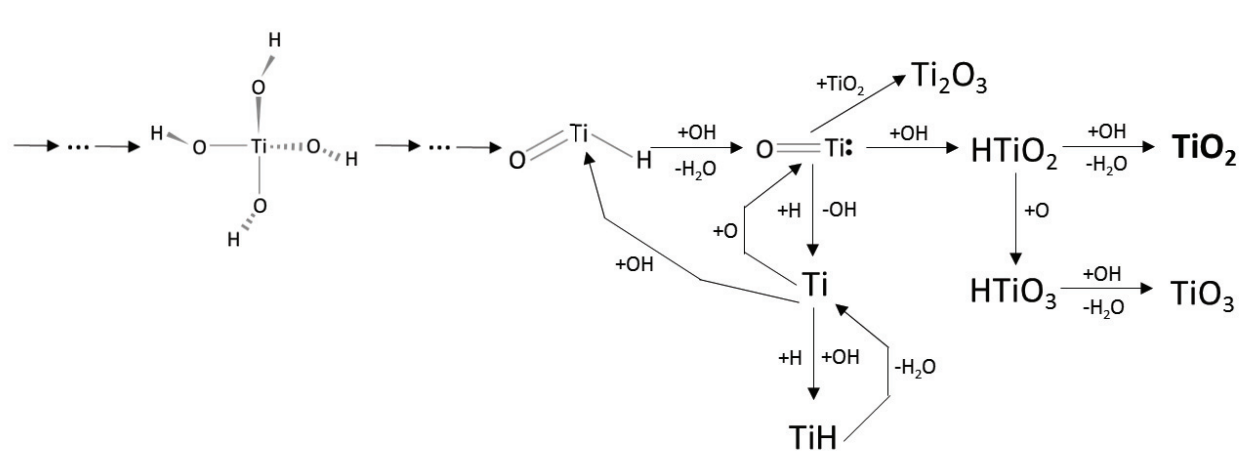

Fig. 24. The stationary points on the potential energy surface (PES) of TTIP and main transformation reactions routes of TTIP in $\mathrm{H}_{2} / \mathrm{O}_{2} / \mathrm{Ar}$ flame. (A): The B3LYP calculations predict the effect activation barrier of the TTIP hydrolysis to be ca. $10 \mathrm{kcal} / \mathrm{mol}$. (B): The energy barrier of TTIP thermal decomposition is as large as $85-87 \mathrm{kcal} / \mathrm{mol}$. (C): The scheme of the main transformation reactions of TTIP in H2/O2/Ar flame. (A and B: reprinted, C: adapted, with permission from Shmakov et al. [571]). 

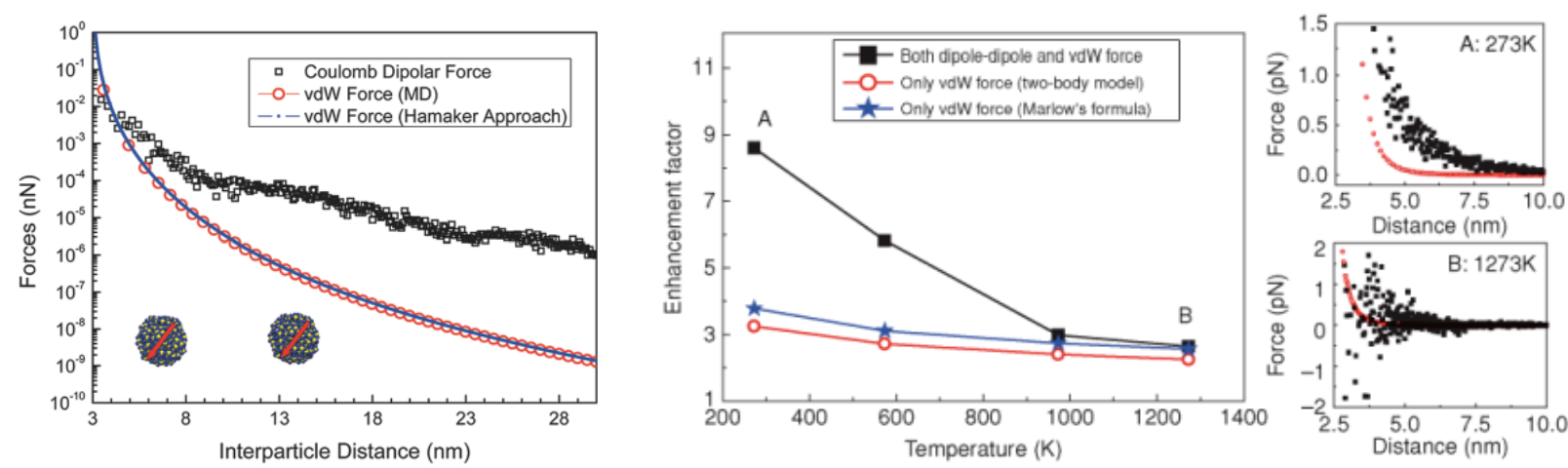

Fig. 25. (a) Comparison between van der Waals forces and dipole-dipole forces between two cooriented $3 \mathrm{~nm} \mathrm{TiO}_{2}$ nanoparticles during the approach process. (b) Influence of dipole-dipole interaction on enhancement factors of coagulation at different temperatures. Subplots A and B show the forces between $\mathrm{TiO}_{2}$ nanoparticles at 273 and 1273K, respectively. (Adapted from Yan et al. [590] and Zhang et al.[591] with permissions). 

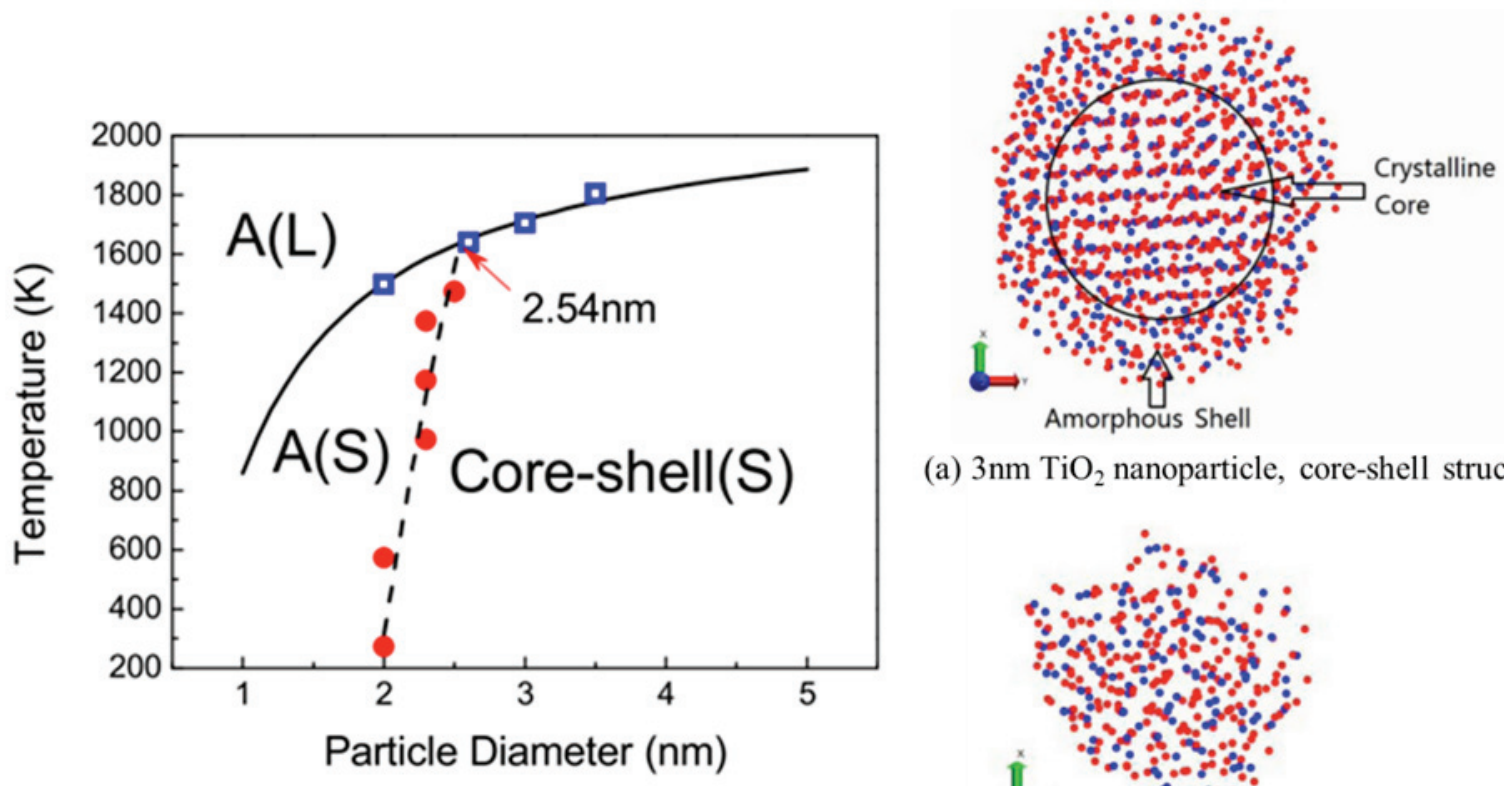

(a) $3 \mathrm{~nm} \mathrm{TiO}$ nanoparticle, core-shell structure

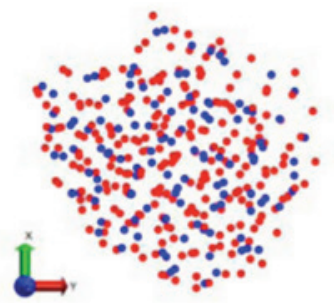

(b) $2 \mathrm{~nm} \mathrm{TiO}{ }_{2}$ nanoparticle, amorphous

Fig. 26. Map of grain structure morphologies of $\mathrm{TiO}_{2}$ for different diameters and temperatures; A stands for amorphous morphology; Core-shell stands for crystalline-core-amorphous-shell morphology; L stands for liquid phase; and $\mathrm{S}$ stands for solid phase. Inserted figure (a) shows a crystalline-core-amorphous-shell structure of a 3nm nanoparticle and (b) shows an amorphous structure of a $2 \mathrm{~nm}$ nanoparticle. (Adapted from Zhang et al. [593] with permissions). 


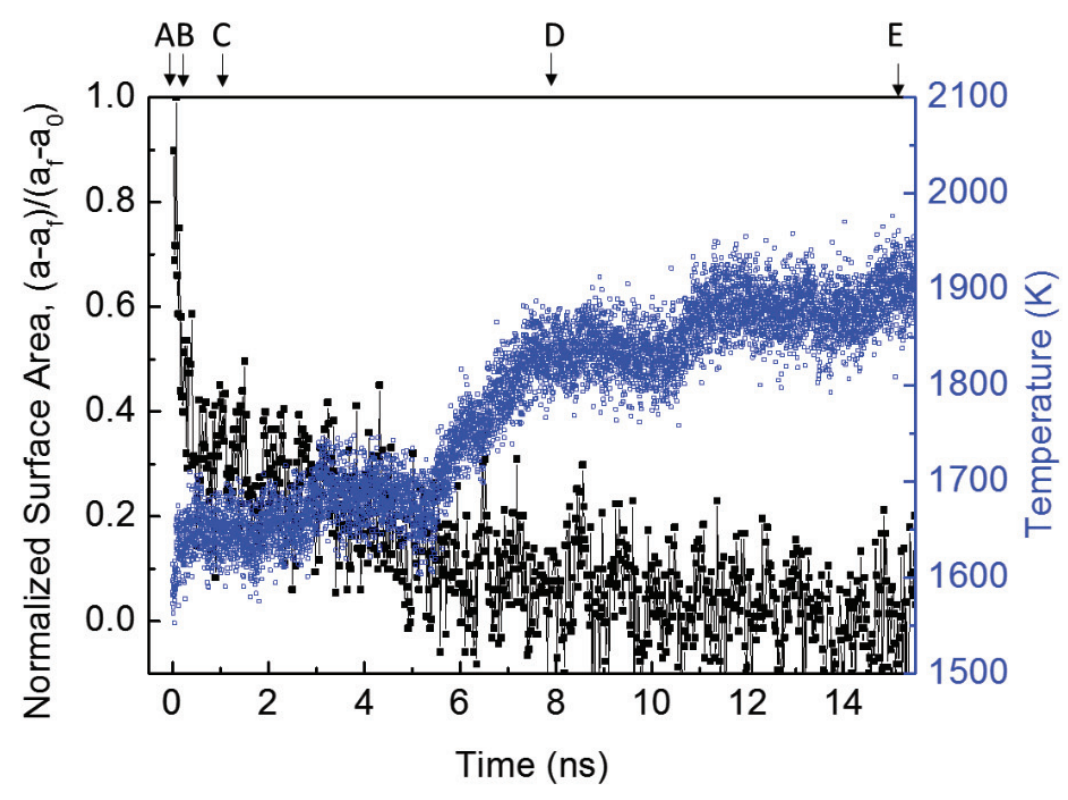

$A: \mathrm{t}=0 \mathrm{~ns}$

$B: t=0.5 n s$
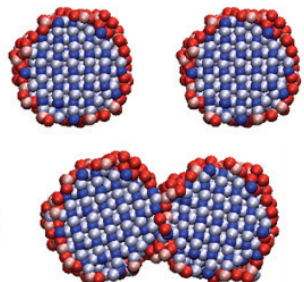

$\mathrm{C}: \mathrm{t}=1 \mathrm{~ns}$

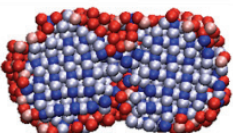

D: $t=8 n s$

$E: t=15 n s$

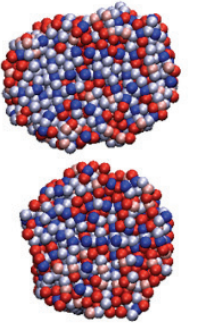

Fig. 27. The normalized surface area and temperature during the sintering process of two $3 \mathrm{~nm}$ TiO2 nanoparticles. Snapshots of nanoparticles at $\mathrm{t}=0,0.5,1,8$ and $15 \mathrm{~ns}$ are shown in the inserted pictures where blue and white spheres are $\mathrm{O}$ and $\mathrm{Ti}$ atoms, respectively, in the certain crystalline region; red and pink spheres are $\mathrm{O}$ and $\mathrm{Ti}$ atoms, respectively, in the outer amorphous region. 